\title{
Dose and Position Measurements using a Novel 4D In Vivo Dosimetry System
}

\author{
by
}

Amanda Cherpak

B.Sc. (Saint Francis Xavier University) 2005

M.Sc. (Carleton University) 2007

A thesis submitted to the

Faculty of Graduate Studies and Research

in partial fulfillment of the requirements

for the degree of

Doctor of Philosophy

Ottawa-Carleton Institute for Physics

Department of Physics, Carleton University

Ottawa, Ontario, Canada

September 2011

Copyright $\mathcal{O}$ 2011, Amanda Cherpak 
Library and Archives

Canada

Published Heritage

Branch

395 Wellington Street

Ottawa ON K1A ON4

Canada
Bibliothèque et

Archives Canada

Direction du

Patrimoine de l'édition

395 , rue Wellington

Ottawa ON K1A ON4

Canada
Your file Votre référence

ISBN: 978-0-494-87748-7

Our file Notre référence

ISBN: $978-0-494-87748-7$

\section{NOTICE:}

The author has granted a nonexclusive license allowing Library and Archives Canada to reproduce, publish, archive, preserve, conserve, communicate to the public by telecommunication or on the Internet, loan, distrbute and sell theses worldwide, for commercial or noncommercial purposes, in microform, paper, electronic and/or any other formats.

The author retains copyright ownership and moral rights in this thesis. Neither the thesis nor substantial extracts from it may be printed or otherwise reproduced without the author's permission.
AVIS:

L'auteur a accordé une licence non exclusive permettant à la Bibliothèque et Archives Canada de reproduire, publier, archiver, sauvegarder, conserver, transmettre au public par télécommunication ou par l'Internet, prêter, distribuer et vendre des thèses partout dans le monde, à des fins commerciales ou autres, sur support microforme, papier, électronique et/ou autres formats.

L'auteur conserve la propriété du droit d'auteur et des droits moraux qui protege cette thèse. $\mathrm{Ni}$ la thèse ni des extraits substantiels de celle-ci ne doivent être imprimés ou autrement reproduits sans son autorisation.
In compliance with the Canadian Privacy Act some supporting forms may have been removed from this thesis.

While these forms may be included in the document page count, their removal does not represent any loss of content from the thesis.
Conformément à la loi canadienne sur la protection de la vie privée, quelques formulaires secondaires ont été enlevés de cette thèse.

Bien que ces formulaires aient inclus dans la pagination, il n'y aura aucun contenu manquant. 
To my godfather, Francis 'Frickie' McDonald, who has been there every step of the way. We get to choose few members of our family. I count my parents' choice to have you in mine as one of my life's greatest blessings. This work is also dedicated to the memory of my grandmother, Mary Cherpak. 


\section{AbSTRACT}

This work presents a comprehensive characterization of the dosimetric and position measurement characteristics as well as clinical implementation of a novel fourdimensional in vivo dosimetry system, RADPOS. Preliminary dose and position measurements were first conducted to evaluate any deviation from known characteristics of metal-oxide semiconductor field-effect transistors, MOSFETs, and electromagnetic positioning systems when they are used alone. The system was then combined with a deformable tissue equivalent lung phantom to simulate respiratory-induced tumour motion and lung deformation and to evaluate the potential use of the system as an effective quality assurance tool for $4 \mathrm{D}$ conformal radiotherapy. The final phase of testing involved using the RADPOS 4D in vivo dosimetry system in two different clinical trials. The first involved characterizing the breathing patterns of lung cancer patients throughout the course of treatment and measuring inter-fraction variations in skin dose. Within this framework, the feasibility of general use of the RADPOS system on patients during daily treatment fractions was also assessed. The second trial involved a modified RADPOS detector that contained a MOSFET array, allowing for dose measurements at five different points. This detector was used to measure dose and position in the prostatic urethra throughout seed implantation for transperineal interstitial permanent prostate brachytherapy.

It has been found that the dosimetric response is similar to that of a microMOSFET, when used alone, aside from a slightly higher variation in angular response. Position measurements can be obtained with an uncertainty of $\pm 2 \mathrm{~mm}$ when the detector remains within a specific optimal volume with respect to the magnetic field 
transmitter and when interfering metal objects are kept at least $200 \mathrm{~mm}$ away. Combining the RADPOS system with a deformable lung equivalent phantom allowed for efficient quality assurance of $4 \mathrm{D}$ radiation therapy, as agreement between dose measurements and treatment plan calculations was within $5 \%$ for both free breathing and adaptive treatment deliveries and position measurements were accurate and consistent between the CT and treatment delivery rooms. The two clinical trials demonstrated that the RADPOS system can be used during daily treatments without any disruption to the treatment schedule or discomfort to patients. The lung patient study found significant deviations in external surface motion which emphasize a need for continued position monitoring. Average measured dose values were in agreement with treatment plan dose calculations for the majority of points. The second clinical trial highlighted deviations from calculated treatment plan values as well as changes in position due to needle placement, swelling, and other internal motion as well as changes due to the TRUS probe. These changes were found to be significant in several cases and should therefore be quantified to evaluate influence on dose distributions. 


\section{ACKNOWLEDGEMENTS}

It is hard to believe that after four years, my Ph.D. research is coming to a close. I feel that on the title page of this thesis, there should be not only my name but the names of all of the people without whom this work would not have been completed. First and foremost, I must express my sincere gratitude to my supervisor, Dr. Joanna Cygler. To say that she has gone above and beyond the requirements of any supervisor is a great understatement. She has sacrificed evenings, weekends, and many hours in between to help me through this process and to ensure my success. The impact of her guidance extends far beyond this thesis and has shaped me as both a person and a student. I must also thank Dr. Dave Rogers for his valuable advice over the years and for setting such an inspiring example. Above all, I thank him for graciously sharing his wife, Dr. Cygler, with me for the past six years.

I would like to thank all of my friends in the Physics Department and at the Cancer Centre for making my time here so memorable. I have met many great friends here and I cherish their support and all of the laughs along the way. This work has been made possible through the help of several other people, in particular Ron Romaine and Steve Andrusyk. I am truly grateful for their endless patience, enthusiasm, and cooperation.

Finally, I would like to thank my family and my boyfriend Morgan. To my sisters, thank you for sharing all of the ups and downs of this journey with me. Your faith and confidence in me has been never ending. To my boyfriend Morgan, thank you for always being in my corner and for believing in me when I didn't believe in myself. You always said it would all work out. You were right. To my parents, thank you for everything. I 
appreciate every sacrifice you have made and every gift you have given. They are too endless to count. Thank you. 


\section{STATEMENT OF ORIGINALITY}

This thesis describes the results of the author's research conducted primarily at the Ottawa Hospital Cancer Centre during the course of the Ph.D. program of Carleton University. Most of these results have been published in either the Medical Physics or Radiotherapy and Oncology journals, and have been presented at both national and international conferences. The details of where the results of these publications may be found in the thesis, along with the author's contributions, are provided below:

I. A.J. Cherpak, W. Ding, A. Halil, and J.E. Cygler, "Evaluation of a novel 4D in vivo dosimetry system.” Med. Phys. 36, 1672-1679 (2009).

- The results of this paper constitute the majority of Chapter 2, which discusses a characterization of the dose and position measuring capabilities of the RADPOS system.

- The author performed all of the measurements, prepared the manuscript for publication, and made the necessary revisions following the review process all under the supervision of Dr. Joanna Cygler.

- The results from this paper were presented by the author at the 2007 COMP conference and by Dr. Joanna Cygler at the 2007 AAPM conference.

II. A.J. Cherpak, M. Serban, J. Seuntjens, J.E. Cygler, “4D Dose-position verification in radiation therapy using the RADPOS system in a deformable lung phantom." Med Phys 38, 179-187 (2011). 
- The results of this paper constitute the majority of Chapter 3, which involves combining the RADPOS system with a deformable lung equivalent phantom for quality assurance of $4 \mathrm{D}$ treatment delivery.

- The co-authors performed all of the measurements together. The author was primarily responsible for RADPOS measurements and analysis as well as preparation of the manuscript for publication under the supervision of Dr. Joanna Cygler. Final edits and revisions were made in collaboration with co-authors.

- Various portions of the results from this paper were presented by the author at the 2007 COMP conference and by Dr. Joanna Cygler at the 2007 AAPM conference and 2008 ESTRO conference.

III. A.J. Cherpak, J.E. Cygler, S. Andrusyk, J. Pantarotto, R. MacRae, G. Perry, "Clinical use of a novel in vivo 4D monitoring system for simultaneous patient motion and dose measurements." Article submitted to Radiotherapy and Oncology.

- The results of this paper constitute the majority of Chapter 4, which involves measurements of patient motion and dose during external beam treatment for lung cancer.

- The author performed all patient measurements. Experiment design, data analysis and manuscript preparation were conducted by the author under the supervision of Dr. Joanna Cygler. 
- Various portions of the results from this paper were presented by the author at the 2009 and 2010 COMP conferences and 2010 ESTRO conference.

IV. A.J. Cherpak, J.E. Cygler, C. E., G. Perry, "Real-time measurement of urethral dose and position during permanent seed implantation for prostate brachytherapy."

- The results of this work constitute the majority of Chapter 5 , which involves the use of a modified RADPOS detector with a MOSFET array for measurements of urethral dose and motion during seed implants for transperineal interstitial permanent prostate brachytherapy.

- All patient measurements were conducted in an operating room by the author and Dr. Joanna Cygler in collaboration with the radiation oncologist and nurse. Data analysis and manuscript preparation was conducted by the author under the supervision of Dr. Joanna Cygler.

- The results of this chapter were presented by the author at the 2011 COMP conference. A manuscript is also in preparation for submission. 


\section{TABLE OF CONTENTS}

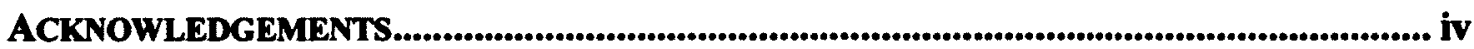

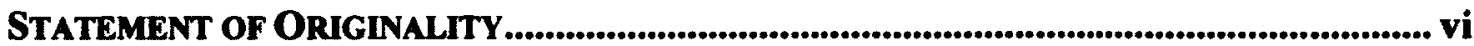

Table of Contents ..................................................................................................................... x

List of Tables ................................................................................................................................ xiv

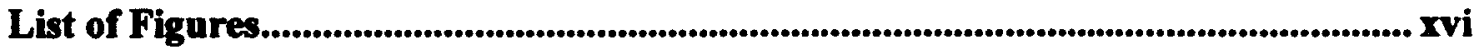

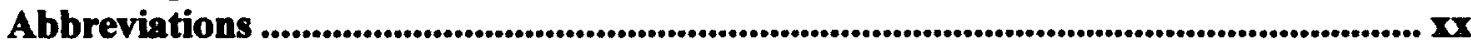

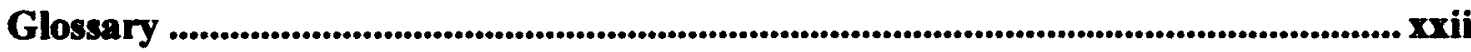

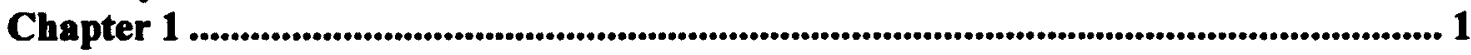

Introduction to the RADPOS system ............................................................................ 1

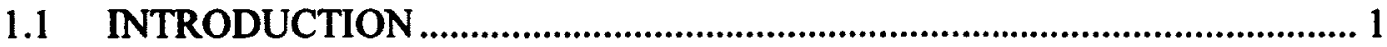

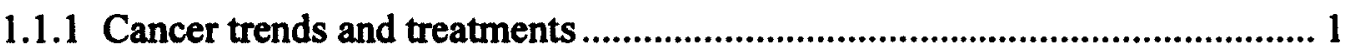

1.1.2 Uncertainties in patient treatment .......................................................... 3

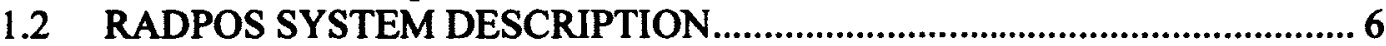

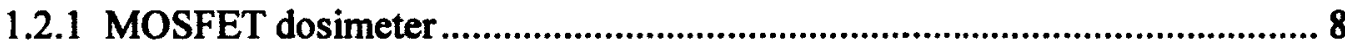

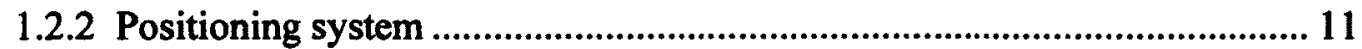

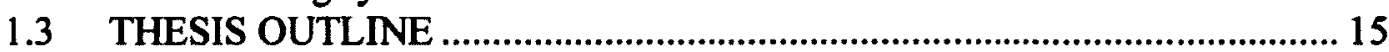

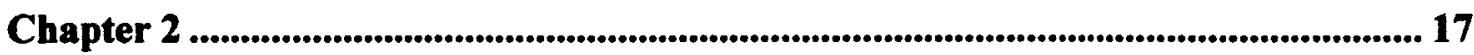

Evaluation of a novel 4D in vivo dosimetry system ............................................. 17

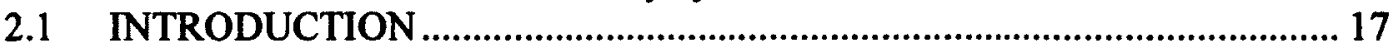

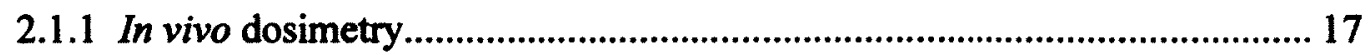

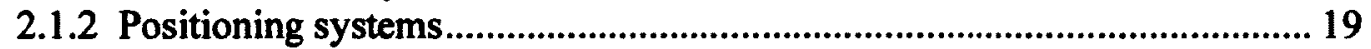

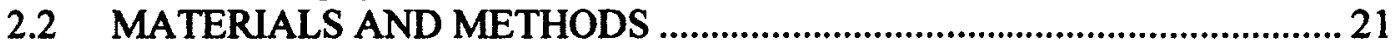

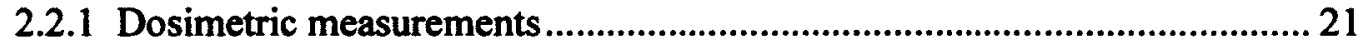

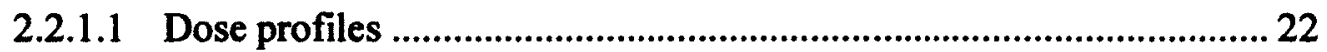

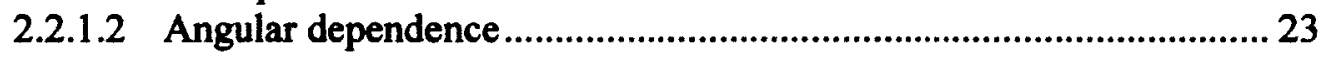

2.2.1.3 Field size dependence ................................................................... 23

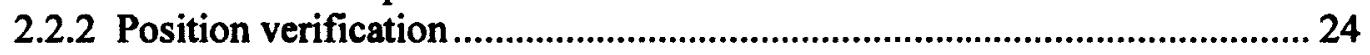

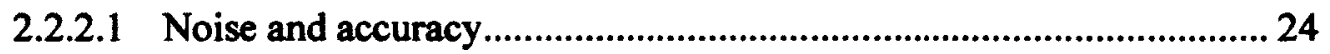

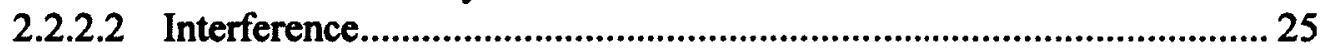

2.2.2.3 Preliminary clinical evaluation.................................................. 27

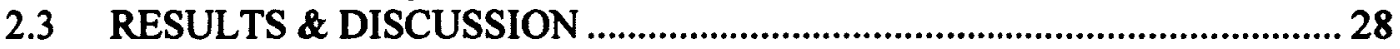

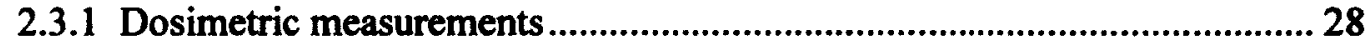

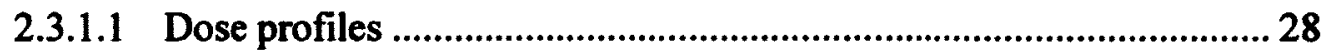

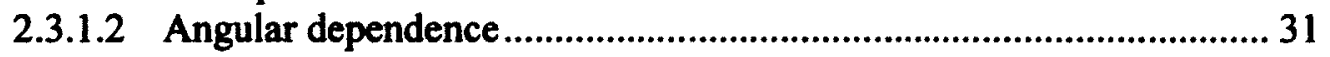

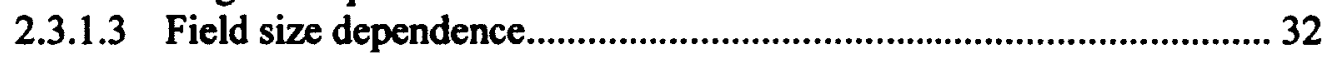

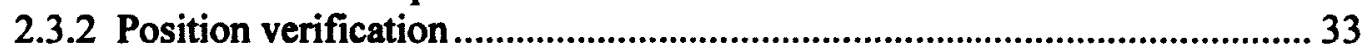

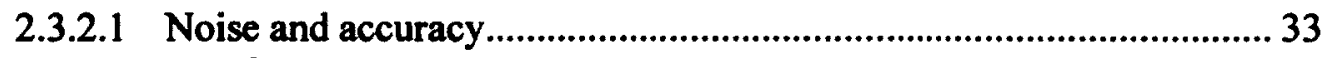

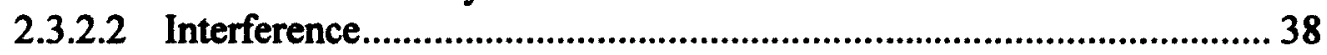

2.3.2.3 Preliminary clinical evaluation.................................................... 42

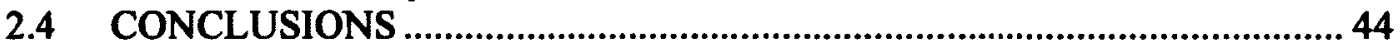


Chapter 3 45

4D Dose-position verification in radiation therapy using the RADPOS system in a deformable lung phantom

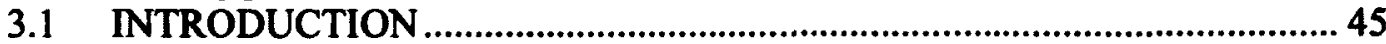

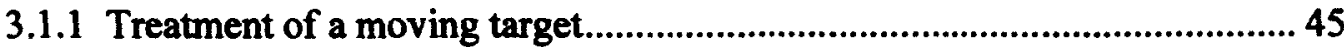

3.1.2 Verification of delivery methods .......................................................... 46

3.2 MATERIALS AND METHODS …........................................................... 48

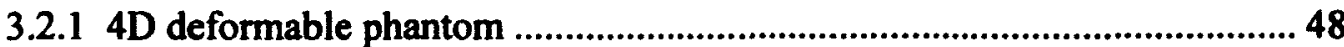

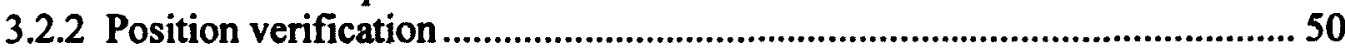

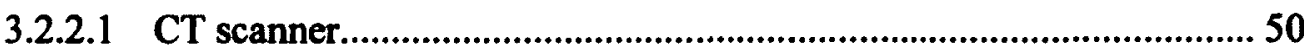

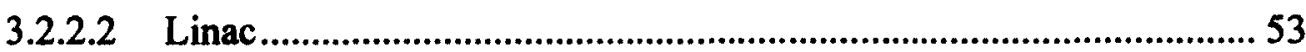

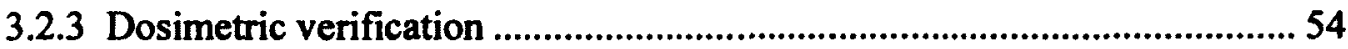

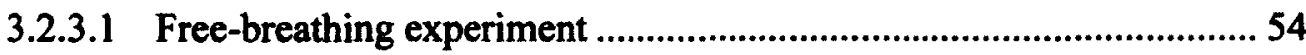

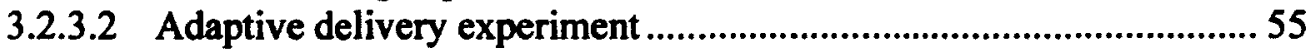

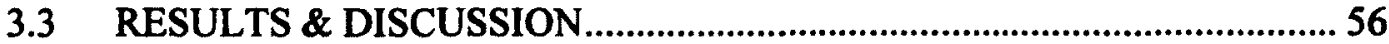

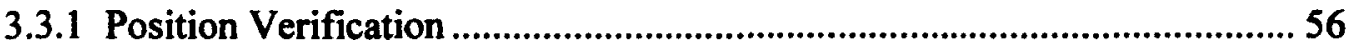

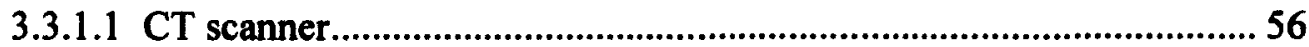

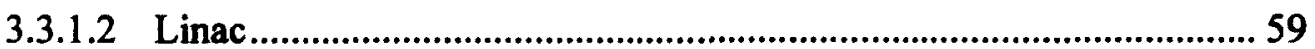

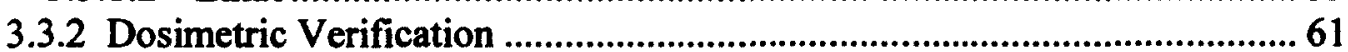

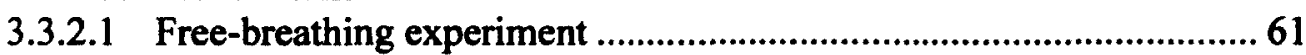

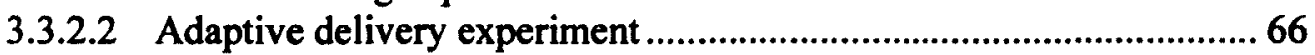

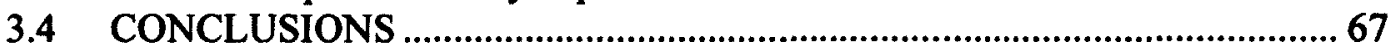

Chapter 4 ................................................................................................................................. 69

Real-time measurements of patient motion and dose during external beam radiation

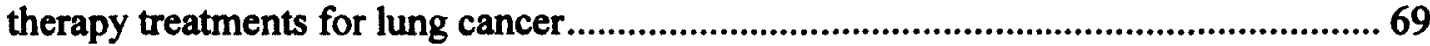

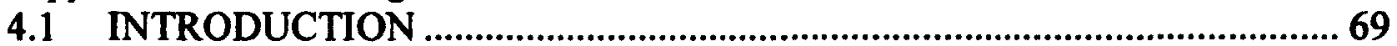

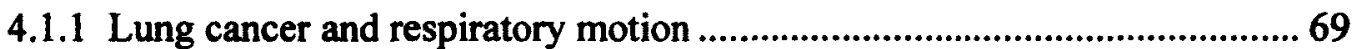

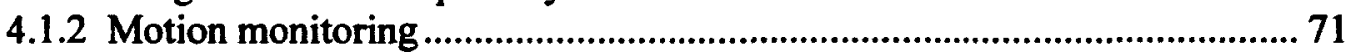

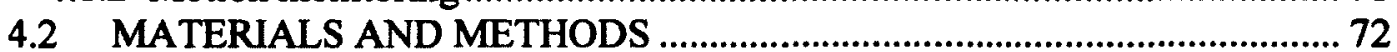

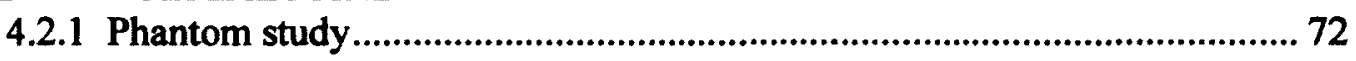

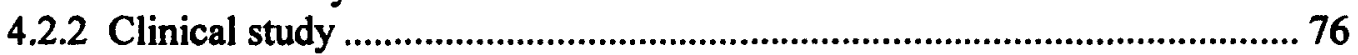

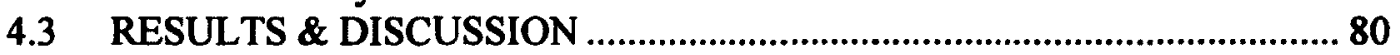

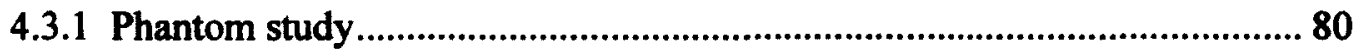

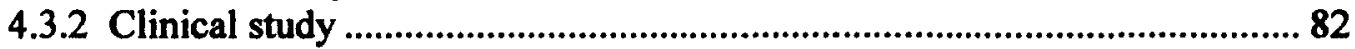

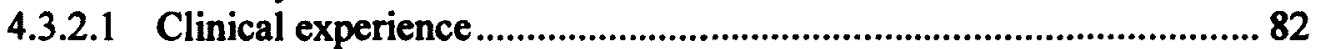

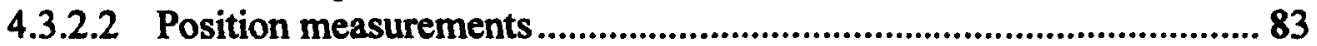

4.3.2.3 Dose measurements........................................................................ 99

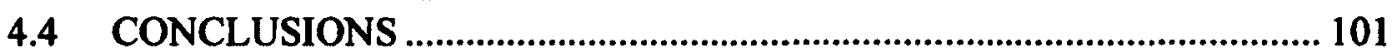

Chapter 5 ........................................................................................................................... 103

Real-time measurement of urethral dose and position during permanent seed

implantation for prostate brachytherapy ............................................................. 103

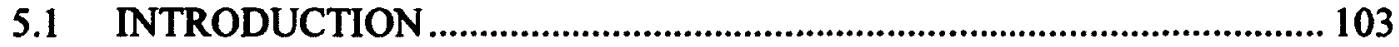

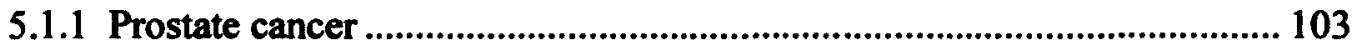

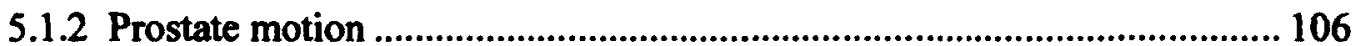

5.1.3 In vivo dosimetry for prostate brachytherapy ..................................... 109

5.2 MATERIALS AND METHODS ...................................................... 111 


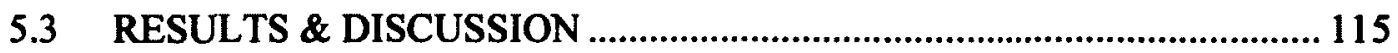

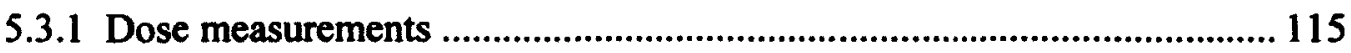

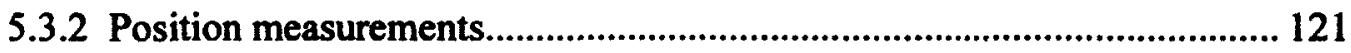

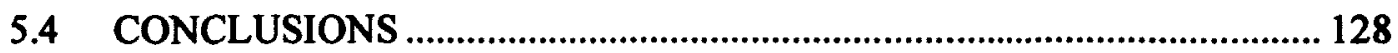

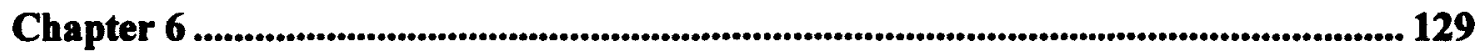

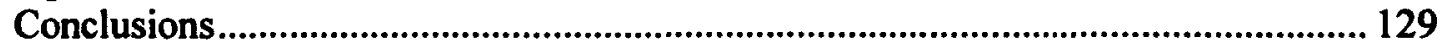

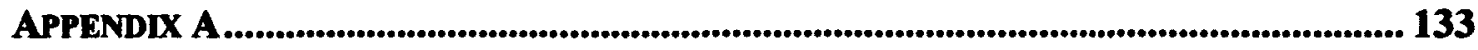

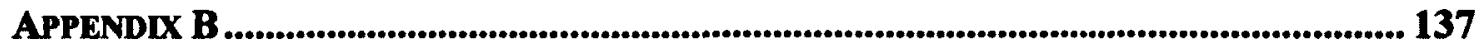

REFERENCES.....................................................................143 


\section{LIST OF TABLES}

Table 2.1 Summary of published results on optimal range and uncertainty for various electromagnetic positioning systems. ${ }^{[27,40-42,47]}$

Table 3.1. RADPOS displacement vector magnitudes in the three piston positions (EOE, MOI, EOI) measured by the RADPOS system on the CT couches and as determined from the CT image set. Measurements were taken twice, the first time with the tumour at a more superior position, further away from the piston (location 1), and a second time with the tumour closer to the piston of the phantom (location 2).

Table 3.2. The displacement vector magnitudes of the two detectors inside the phantom measured by the RADPOS system on the CT and linac couches. The displacement vector for each trial is shown (ex. $r_{C T}={\sqrt{x_{C T}{ }^{2}+y_{C T}{ }^{2}+z_{C T}{ }^{2}}}^{\text {}}$ ) as well as the difference between the measurements made on the two different couches $\left(\Delta r=\sqrt{\left(x_{C T}-x_{\text {linax }}\right)^{2}+\left(y_{C T}-y_{\text {thax }}\right)^{2}+\left(z_{C T}-z_{\text {linax }}\right)^{2}}\right)$

Table 3.3. RADPOS-measured dose values and treatment plan doses for the EOE and EOI phases of the free-breathing experiment. Data are shown for a) Detector 1: inside the tumour and b) Detector 2, inside the lung portion of the phantom. 65

Table 3.4. RADPOS-measured dose values for the EOE, MOI, and EOI phases of the adaptive treatment experiment. Data are shown for Detector 1, inside of the tumour. ... 67

Table 4.1. Results from Student $t$ test comparing average amplitude and period measured during treatment to values measured at the time of the 4DCT. Results with a p-value less than $\alpha=0.05$ are considered significant and are shown in bold.

Table 4.2. Results of the correlation analysis between the number of days since a patient's $4 D C T, \Delta_{4 D C T}$, and daily average amplitude, $\hat{z}_{D 1}$, daily average period, $\hat{T}_{D 1}$, and standard deviations of these values, $\sigma_{z_{D 1}}$ and $\sigma_{T_{D 1}}$. Moderate to very strong correlations $(0.4<|r|>1.0)$ are show in bold. 90

Table 5.1. Percent difference between the total integral dose values measured with the probe in place, $D_{\text {probe, }}$ and without the probe in place, $D_{n o}$ probe, for those MOSFETs determined to be within the prostate. 120

Table 5.2. Range of average change in position among all patients during treatment planning, during implantation, and finally due to removal of the TRUS probe. 


\section{LIST OF FIGURES}

Figure 1.1 Trends in new cases for all cancers and ages, for a) males and b) females. The bottom curve shows increase in cancer rates due to changes in cancer risk or diagnostic practices, the middle curve shows changes attributed to population growth, while the top curve shows changes due to the aging population. ${ }^{[1]}$

2

Figure 1.2 Various tumour and target definitions used throughout the treatment planning process. 4

Figure 1.3 Schematic of the RADPOS dosimetry system. 7

Figure 1.4 RADPOS detector. The MOSFET dosimeter is at the tip of the wire and the positioning sensor is located $8 \mathrm{~mm}$ away. Several of the detectors also have a marker at the end of the wire, above the MOSFET, that is visible on CT images. 7

Figure 1.5 MOSFET structure. Diagram adapted from M Soubra, J Cygler, and G Mackay, "Evaluation of dual-bias-dual-metal-oxide-silicon semiconductor field effect transistor detector as radiation dosimeter," Med Phys. 21, 567-572 (1994). ${ }^{[26]}$................ 9

Figure 1.6 Change in threshold voltage of MOSFET with exposure to radiation........... 10

Figure 1.7 The RADPOS DC magnetic field transmitter and coordinate system. ........... 12

Figure 1.8 Coordinate systems of a) the patient, b) the RADPOS system, and c) the radiation beam. The RADPOS axes $(x, y, z)$ are defined by the placement of the transmitter, which has been kept consistent with respect to the patient (left/right, superior/inferior, and anterior/posterior axes) and the radiation beam (cross-plane, inplane, and depth axes) throughout all testing. 14

Figure 2.1. Direction of scans in the $\mathrm{x}, \mathrm{y}$, and $\mathrm{z}$ directions. The origin was defined as a point $200 \mathrm{~mm}$ away from the transmitter, along the $x$ direction. 25

Figure 2.2. Set-up for material interference tests........................................................ 26

Figure 2.3. Quasar Respiratory Motion Phantom ${ }^{\mathrm{TM}}$. The phantom has two moving components, the translation stage and the chest wall platform. The translation stage moves horizontally, and has been always set-up to move in the superior/inferior direction for this work. 28

Figure 2.4. In-air cross-plane dose profiles measured with the RADPOS prototype and the RFA300 and diode for a) ${ }^{60} \mathrm{Co} \mathrm{b)} 6 \mathrm{MV}$ and c) $18 \mathrm{MV}$ beams. The dashed line in each figure represents diode measurements and the solid line represents RADPOS measurements. Error bars represent the standard deviation of the data for 3 measurements. The detector was positioned at an SDD of $100 \mathrm{~cm}$.

Figure 2.5. Relative change in the threshold voltage, $\triangle V$ th, of the RADPOS prototype plotted as a function of probe angle (bubble down $=0^{0}$ ). Energy: ${ }^{60} \mathrm{Co}$, field size: $10 \times 10$ $\mathrm{cm}^{2}$, polystyrene phantom, $\mathrm{SSD}=80 \mathrm{~cm}$, depth $1 \mathrm{~cm}$. 
Figure 2.6. Relative calibration coefficients measured at field sizes of $6 \times 6 \mathrm{~cm}^{2}$ to $25 \times 25$ $\mathrm{cm}^{2}$ for standard and high sensitivity MOSFETs.

Figure 2.7. a) Difference between RADPOS and RFA positions taken along the $x$ (inplane) axis, $y$ (cross-plane) axis and $z$ (depth) axis. b) Boundaries for optimal volume for RADPOS position measurements based on data shown in part a of the figure. With the origin defined at a point $200 \mathrm{~mm}$ away from the transmitter along the $x$ axis, these boundaries extend from $x=-50$ to $320 \mathrm{~mm}, y=-215$ to $215 \mathrm{~mm}$, and $z=-100$ to 100 $\mathrm{mm}$. 36

Figure 2.8. Results from metal interference test with $25 \times 25 \mathrm{~cm}^{2}$ samples that caused an effect on the RADPOS position (aluminium, brass, lead, steal). 38

Figure 2.9. Respiratory motion produced by the Quasar phantom, as measured by the RADPOS detector. The phantom was moving according to sample patient files that displayed a) deep breathing and b) irregular breathing. 43

Figure 3.1. Schematic of the 4D deformable lung phantom and RADPOS detectors used in this work. Detectors 3 (on couch) and 4 (CT zero) remained stationary and were used as reference points throughout the experiment. The thick arrow showing piston travel is also used to indicate the deformation of the phantom balloon (shown as the dotted outline) for various breathing phases. 49

Figure 3.2. Deformable lung phantom on the couch of the CT scanner. To investigate interference from the CT on the RADPOS-read positions, the couch was moved to position the phantom as far as $2 \mathrm{~m}$ away from the CT bore to when the phantom was in the middle of the bore. 52

Figure 3.3. Gantry rotation about an axis parallel to the treatment couch 54

Figure 3.4. Reproducibility of RADPOS positions with the piston in EOE, MOI, and EOI states for a) Detector 1, inside the tumour and b) Detector 2, inside the lung tissue. For each detector, the positions were zeroed at the original EOE position. The average and standard deviation of the position at each stage is shown on the plot. For all trials, the position of each detector in a given phase remained within $0.9 \mathrm{~mm}$ of the other positions recorded for that detector and phase. 57

Figure 3.5. Difference of relative RADPOS position readings at various angles from reading at gantry angle $(\mathrm{GA})=120^{\circ}$, (eg. $x_{\text {Den,GA }}^{\prime}=\left(\left(x_{D e t, G A}-x_{\text {ref,GA }}\right)-x_{\text {Det1,120 }}\right)$ ) for the detector inside the tumour. Data shown are averaged over all three phases (EOE, MOI, EOI), and error bars represent the standard deviation of the averaged values. 61

Figure 3.6. The beam eye view for treatment delivery with the anterior $0^{\circ}$ beam for a) $1 \mathrm{st}$ state: phantom in EOE phase (position used for planning) and b) 2nd state: phantom in EOI phase (tumour now partially out of primary beam). Dose volume histograms are also shown for the two halves of the treatment: c) EOE and d) EOI. The dashed curve shows the dose to the lung and the solid curve represents the dose to the tumour. 63 
Figure 4.1. Set-up for phantom motion measurements with the RADPOS and a) the Philips Bellows belt and b) the Varian RPM system positioned on top of the Quasar phantom chest wall platform 74

Figure 4.2. Example of how the RADPOS data were shifted to correspond with the RPM data according to the time at which the flat line signal began. 75

Figure 4.3. Placement of RADPOS detectors during 4DCT and treatment delivery. ...... 78

Figure 4.4. Measurements of a 4D phantom's periodic motion by the RADPOS system and a) Phillips Bellows and b) Varian RPM with synchronized time. 82

Figure 4.5. Statistical plot of the daily average a) amplitude in the anterior/posterior direction, $\hat{z}_{D 1}$, and b) period, $\hat{T}_{D 1}$, measured by Detector 1 during treatment fractions compared to the same measurement taken during the 4DCT $\left(\hat{z}_{D 1,4 D C T}\right.$ and $\left.\hat{T}_{D 1,4 D C T}\right) \ldots . .88$

Figure 4.6. Statisical plot of a) $\Delta_{z_{D 1}}=$ (daily max amplitude - daily min amplitude) and b) $\Delta_{T_{D 1}}=$ (daily max period - daily min period) measured Detector 1 during treatment fractions compared to the same measurement taken during the 4DCT $\left(\Delta_{z_{D 1}, 4 D C T}\right.$, $\left.\Delta_{T_{D 1}, 4 D C T}\right)$

Figure 4.7. Example of detector placement on patients with different tumour locations including a) upper lobe left lung/bronchus tumour b) upper lobe right lung/bronchus tumour c) lower lobe left lung/bronchus tumour. .95

Figure 4.8. Results of the cross correlation analysis of data collected simultaneously by the three detectors on each patient. Figure a) shows the results with zero lag, $\rho(0)$, while b) shows the maximum cross correlation coefficient, $\rho_{\max }$ for each data pair.

Figure 4.9. Daily RADPOS dose measurements, $D_{R A D P O S} \pm \sigma_{D_{\text {RaDPOS }} \text {, compared to the }}$ calculated treatment plan dose, $\hat{D}_{T P} \sigma_{D_{T P}}$. For each measurement site, the average treatment plan dose, $\hat{D}_{T P}$, is shown with the horizontal black line, and the upper and lower limits of $\hat{D}_{T P} \sigma_{D_{m}}$ are shown by the blue and red dashed lines respectively.... 100

Figure 5.1. Pelvic anatomy. (National Kidney and Urologic Diseases Information Clearinghouse, NIH-06-3012, Bethesda MD 2006) 104

Figure 5.2. Set-up for transperineal interstitial permanent prostate brachytherapy (TIPPB). ${ }^{[112]}$

Figure 5.3. Transperineal radioactive seed implantation guided by transrectal ultrasound (TRUS) 106

Figure 5.4. RADPOS detector with MOSFET array. 
Figure 5.5. Fluoroscopy image taken during implant procedure. RADPOS marker can be seen inside the Foley catheter balloon.

Figure 5.6. Typical position of RADPOS array with respect to pelvic anatomy during TIPPB procedure.

Figure 5.7. RADPOS dose measurements and calculated treatment plan dose values for Patient 6. 117

Figure 5.8. Comparison of total integral dose calculated from RADPOS dose measurements and treatment plan values. For each patient, the differences for all MOSFET locations were normalized to the maximum dose measured for that patient. 117

Figure 5.9. Comparisons of relative dose measurements taken before and after the TRUS probe was removed for a) Patient 13, b) Patient 7, and c) Patient 16 . In each case, the total integral dose values have been normalized to the maximum integral dose for all MOSFETs with the TRUS probe in place specific for the patient presented. Errors bars are not visible because they are smaller than the symbols shown. 119

Figure 5.10. Position information collected during seed implantation for Patient 6. The root mean squared position $\left(r=\sqrt{\left(x^{2}+y^{2}+z^{2}\right.}\right)$ is shown by the black line and the grey circles represent needle insertion.

Figure 5.11. RADPOS position measurements for Patient 8 taken after implantation was complete. Black squares represent the time of the initial and final MOSFET readings taken for the first measurement period (with TRUS probe in place) and the second measurement period (after the TRUS probe was removed). The change in position from the removal of the probe can be seen in all coordinates at approximately $t=4100 \mathrm{~s} . \ldots 125$

Figure 5.12. Fluoroscopy images taken post-implant once the TRUS probe was removed for Patients a) 8 b) 10 and c) 16. Outlines of seed, probe, and RADPOS marker positions from before the probe was removed are shown to display displacements. Outlines have been registered to the pubic arch. 128 


\section{AbBreviations}

$\begin{array}{ll}\text { EOE } & \text { End of exhale } \\ \text { EOI } & \text { End of inhale } \\ \text { DVH } & \text { Dose volume histogram } \\ \text { CBCT } & \text { Cone-beam computed tomography } \\ \text { MIP } & \text { Maximum intensity projection } \\ \text { MOI } & \text { Middle of inhale } \\ \text { MOSFET } & \text { Metal-oxide semiconductor field-effect transistor } \\ \text { MU } & \text { Monitor unit } \\ \text { NSCLC } & \text { Non-small cell lung carcinoma } \\ \text { PDD } & \text { Percentage depth dose curve } \\ \text { TLD } & \text { Thermoluminescent dosimeter } \\ \text { TRUS } & \text { Transrectal ultrasound probe } \\ \text { TIPPB } & \text { Transperineal interstitial permanent prostate brachytherapy }\end{array}$




\section{GLOSSARY}

Centigray (cGy) Unit of absorbed dose equivalent to [(Joules/kilogram) $\left.\times 10^{-2}\right]$.

Clinical target volume (CTV) Gross tumour volume with extensions to include microscopic spread of disease and regions with a high risk of involvement based on clinical experience. It represents the true extent and location of the tumour.

Computed tomography reference isocenter A point defined on the patient's body during a planning $\mathrm{CT}$ that will be lined up with the machine isocenter during subsequent radiation therapy treatments. The machine isocenter is where the collimator axis and the axis of rotation of the treatment machine gantry intersect.

Dose profile The measurement of delivered dose along the longitudinal or transverse axis of a treatment machine.

Fiducial marker A fixed point of comparison within the patient body.

Gated beam delivery Method of radiation therapy delivery that involves tracking a patient's breathing or direct tumour motion and turning the radiation beam on only when the tumour is believed to be within a specific region.

Gross tumour volume (GTV) Macroscopic volume of tumour that is palpable or visible using imaging modalities.

Image guidance The use of daily images taken with the patient on the treatment couch to ensure accurate patient positioning before treatment is delivered.

Intensity-modulated radiation therapy (IMRT) A radiation therapy technique that involves treating the patient from a number of different directions with beams of nonuniform fluences that have been optimized by a treatment planning system.

Inter-fraction A descriptive term used to refer to variations seen between separate treatment fractions.

Intra-fraction A descriptive term used to refer to variations seen within a single treatment fraction.

In vivo dosimetry Measurement of dose delivered to a patient during radiation therapy delivery.

Irradiated target volume (ITV) Volume receiving a significant dose, based on normal tissue tolerance doses. 
Phantom Material that will absorb and scatter photons in the same way as tissue. Water and wet tissues absorb photons in almost the same way, so water or water equivalent material is often used.

Planning target volume (PTV) Volume that includes the clinical target volume as well as internal and set-up margins to account for patient and tumour movement and set-up uncertainties.

Source-to-surface distance (SSD) Distance between the radiation source and skin or phantom surface.

Therapeutic ratio Ratio of the dose that leads to serious complications in a specific percentage of patients to the dose that gives tumour control in the same percentage of patients. 


\section{Chapter 1}

\section{INTRODUCTION TO THE RADPOS SYSTEM}

\subsection{INTRODUCTION}

\subsubsection{Cancer trends and treatments}

The Canadian Cancer Society predicts that in the year 2011, an average of twenty Canadians will be diagnosed with some type of cancer every hour. ${ }^{[1]}$ This totals over 175,000 new cases by the end of the year, a number that is expected to increase dramatically with our aging population. ${ }^{[1]}$ The effects of aging and population growth on the number of new cases of cancer each year are shown in figure 1.1. This expected increase highlights the need for effective treatment, to reduce recurrences and associated health care costs. 
a)

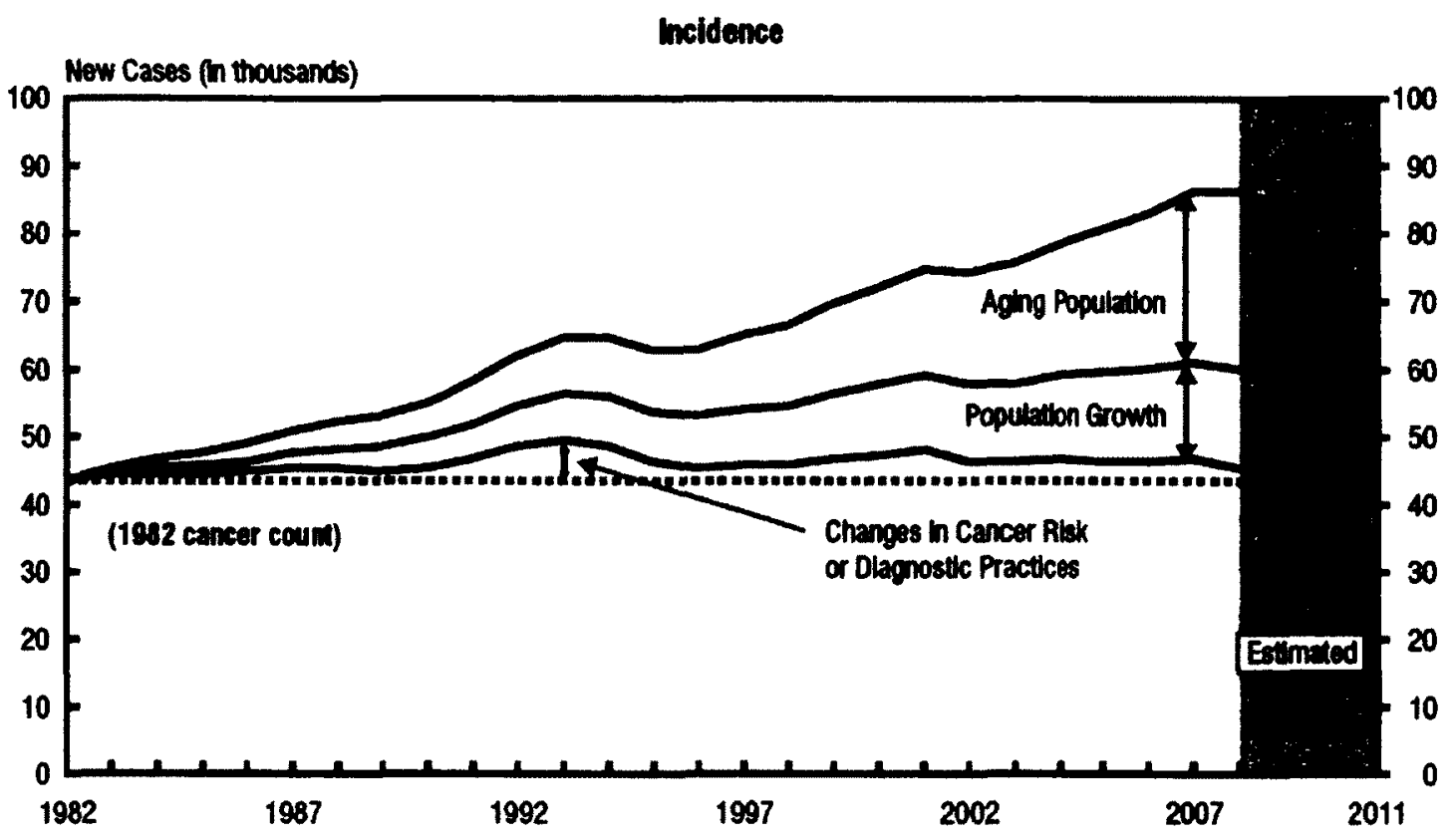

b)

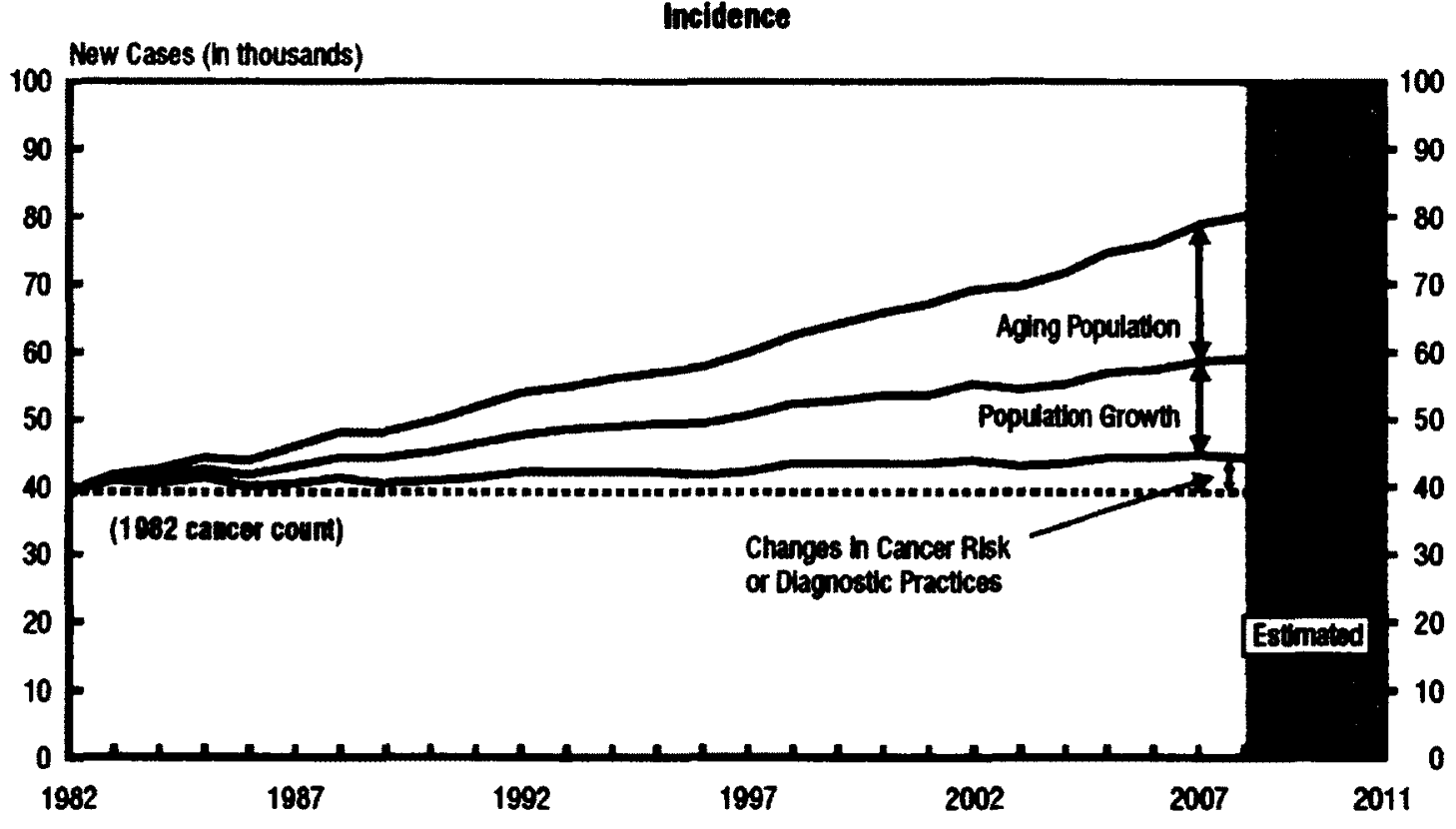

Figure 1.1 Trends in new cases for all cancers and ages, for a) males and b) females. The bottom curve shows increase in cancer rates due to changes in cancer risk or diagnostic practices, the middle curve shows changes attributed to population growth, while the top curve shows changes due to the aging population. ${ }^{\text {[I] }}$ 
There are currently three main methods of treatment for cancer: surgery, chemotherapy, and radiation therapy. Patients often receive more than one type of therapy throughout their disease, with approximately fifty percent of patients undergoing surgery, thirty percent receiving chemotherapy, and thirty percent having radiation therapy treatments. Radiation combats cancer cells by damaging a cell's DNA, destroying its ability to reproduce. It is also capable however of damaging healthy cells through the same mechanisms. Radiation treatment plans aim to deliver highly conformal dose to the tumour while at the same time sparing surrounding healthy tissues and modern radiation therapy technology is in principle capable of delivering such complex treatments. ${ }^{[2]}$ Unfortunately, the patient is not a static object and although sophisticated immobilization devices and breathing techniques are employed, some movement of the tumour exists not only between different treatment fractions (inter-fraction movement), but also during a single treatment (intra-fraction movement). ${ }^{[3-5]}$

\subsubsection{Uncertainties in patient treatment}

Intra-fraction movements can lead to insufficient dose delivered to the treatment target and can also result in an overdose of nearby healthy organs. ${ }^{[3-5]}$ The largest contributors to intra-fraction movement are breathing and cardiac motion, which affect mainly organs in the abdomen and thorax. ${ }^{[3,6]}$ The planning target volume, PTV, which encompasses an extra margin around the clinical target volume, is used to account for uncertainties due to patient positioning and organ motion. 


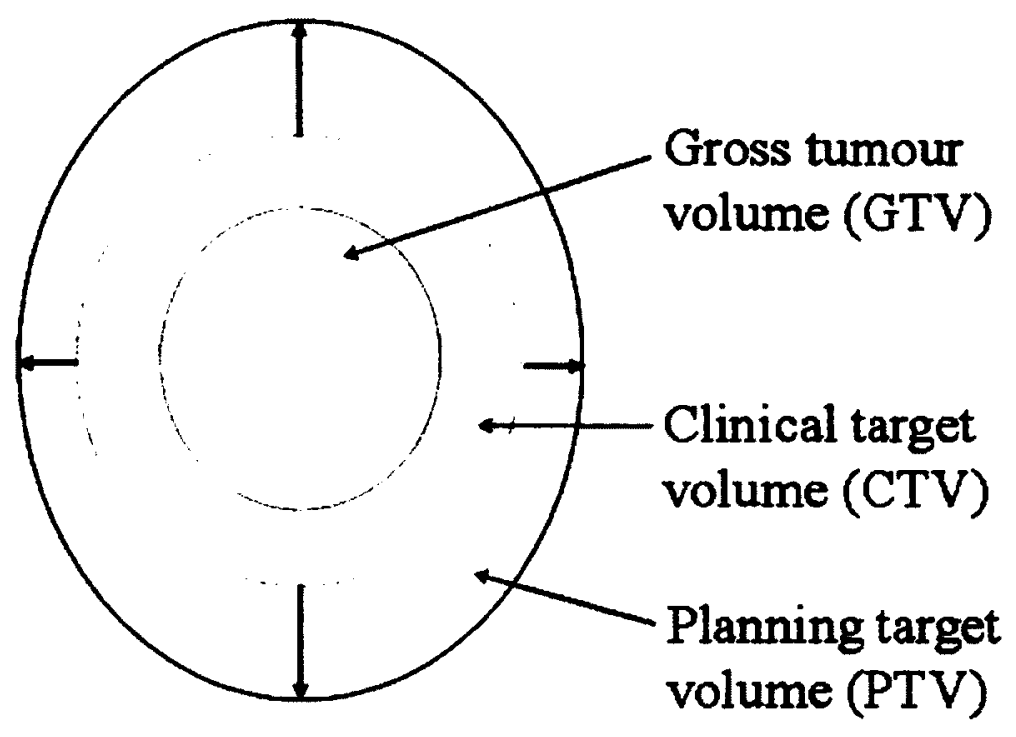

Figure 1.2 Various tumour and target definitions used throughout the treatment planning process.

The definition ${ }^{[7]}$ of the planning target volume is however limited by the presence of nearby organs at risk and other healthy tissue. ${ }^{[8,9]}$ Imaging systems, such as ultrasound, $x$-ray, infrared cameras, portal imaging and computed tomography, can be used to determine tumour position prior to daily treatments, and necessary adjustments can be made to the patient position at that time..$^{[7,10-16]}$ This reduces the risk of set-up errors, which can have significant consequences on delivered dose. ${ }^{[17]}$ It does not, however, account for motion throughout the duration of the treatment, which can be between 15-45 minutes for some image-guided intensity-modulated radiation therapy, IMRT, treatments. ${ }^{[18,19]}$ Continuous monitoring of target and patient position during irradiation can provide valuable information for more complex methods of radiotherapy delivery, which rely on real-time position data, and can identify action levels when the patient or target has moved too much. ${ }^{[20-22]}$ 
Another important part of accurate dose delivery is the measurement of radiation delivered to the patient. Several problems with computer optimized treatment planning systems have been identified, such as inaccurate dose calculations near the surface and at other interfaces between different materials. ${ }^{[23,24]}$ In addition, the treatment machine output may vary during treatment delivery. In vivo dosimeters capable of providing quick and accurate dose information can be a valuable asset to quality assurance of treatment delivery. Real-time dose measurements can identify errors while the patient is still on the treatment table so corrections to the patient position or treatment plan can be made much earlier than with dosimeters that require long processing times, like thermoluminescence dosimeters, TLDs.

To this end, a new four-dimensional (4D) in vivo dosimetry system that combines real-time position monitoring with simultaneous dose measurement has recently been developed. The device is called Radiation Positioning System, RADPOS, and it merges two technologies: an electromagnetic positioning device coupled with a metal-oxide semiconductor field-effect transistor, MOSFET, radiation detector. Initial conceptualization of the device took place at The Ottawa Hospital Cancer Center before the work presented here began, however continued improvements to both the detector and software have been carried out since that time. Development of the RADPOS system has been in collaboration with Best Medical, Ottawa ON, where manufacturing of the device has taken place. The RADPOS system has been patented and is commercially available in Canada. ${ }^{[25]}$ 


\subsection{RADPOS SYSTEM DESCRIPTION}

A schematic of the RADPOS (Best Medical Canada, Ottawa, ON) is shown in figure 1.3. The probe consists of either a high sensitivity (TN-1002RDM) or standard sensitivity (TN-502RDM) microMOSFET dosimeter (Best Medical Canada, Ottawa, ON) and an Ascension 3D Guidance medSAFE electromagnetic positioning tracker (Ascension Technology Corporation, Burlington, VT), which are both mounted on a long flexible wire. To avoid radiation attenuation and disturbance, the positioning sensor, attached to the probe wire, is separated by $8 \mathrm{~mm}$ from the MOSFET dosimeter, as shown in figure 1.4. The position coordinates of the detector are determined by monitoring the response of the sensor to a pulsed 3D magnetic field. This field is generated by a 3DGuidance direct current, DC, magnetic field transmitter which must be attached to the treatment couch, stationary with respect to the patient/phantom coordinate system. The RADPOS probe is connected to a mobile MOSFET reader (TN-RD-16), a 3D-Guidance preamplifier and a 3D-Guidance medSafe electronics unit which are controlled by a host computer using either a direct connection or wireless technology. This allows the user to sample and view dose and position information outside the treatment room in real-time while the patient is on the treatment couch. 


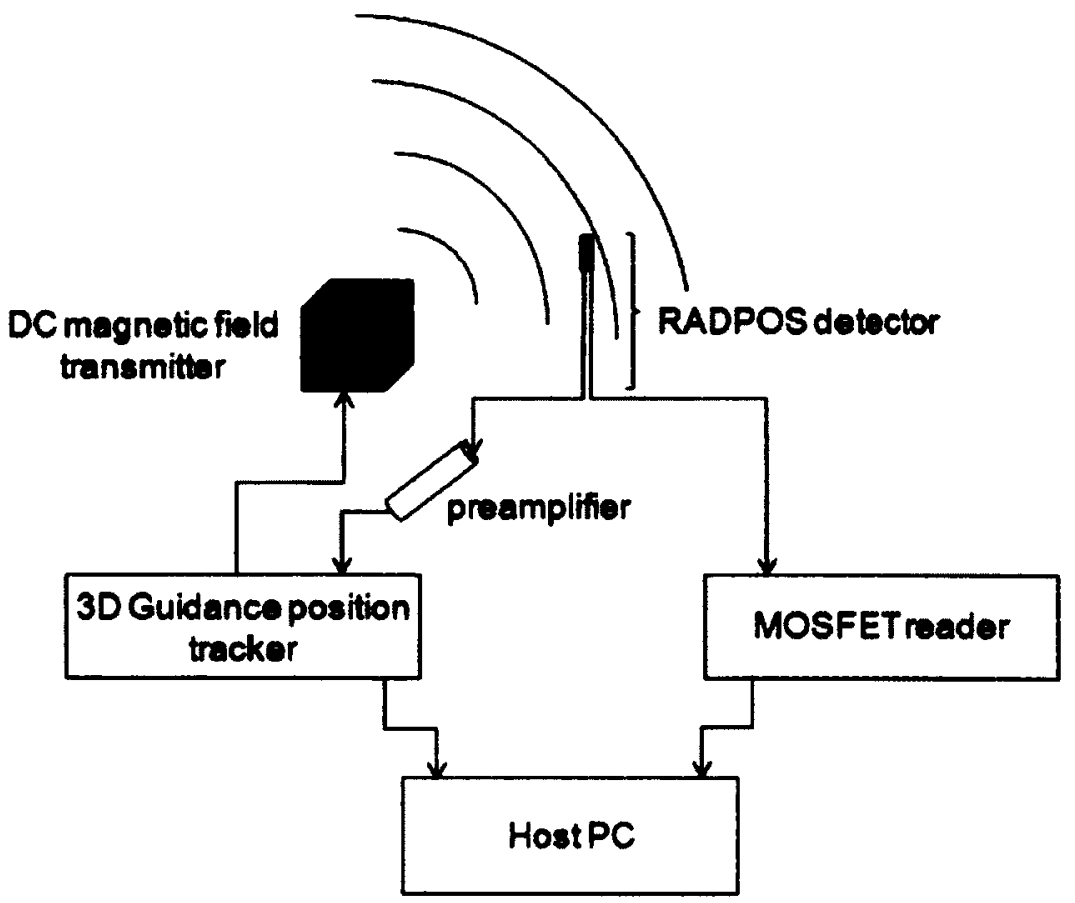

Figure 1.3 Schematic of the RADPOS dosimetry system.

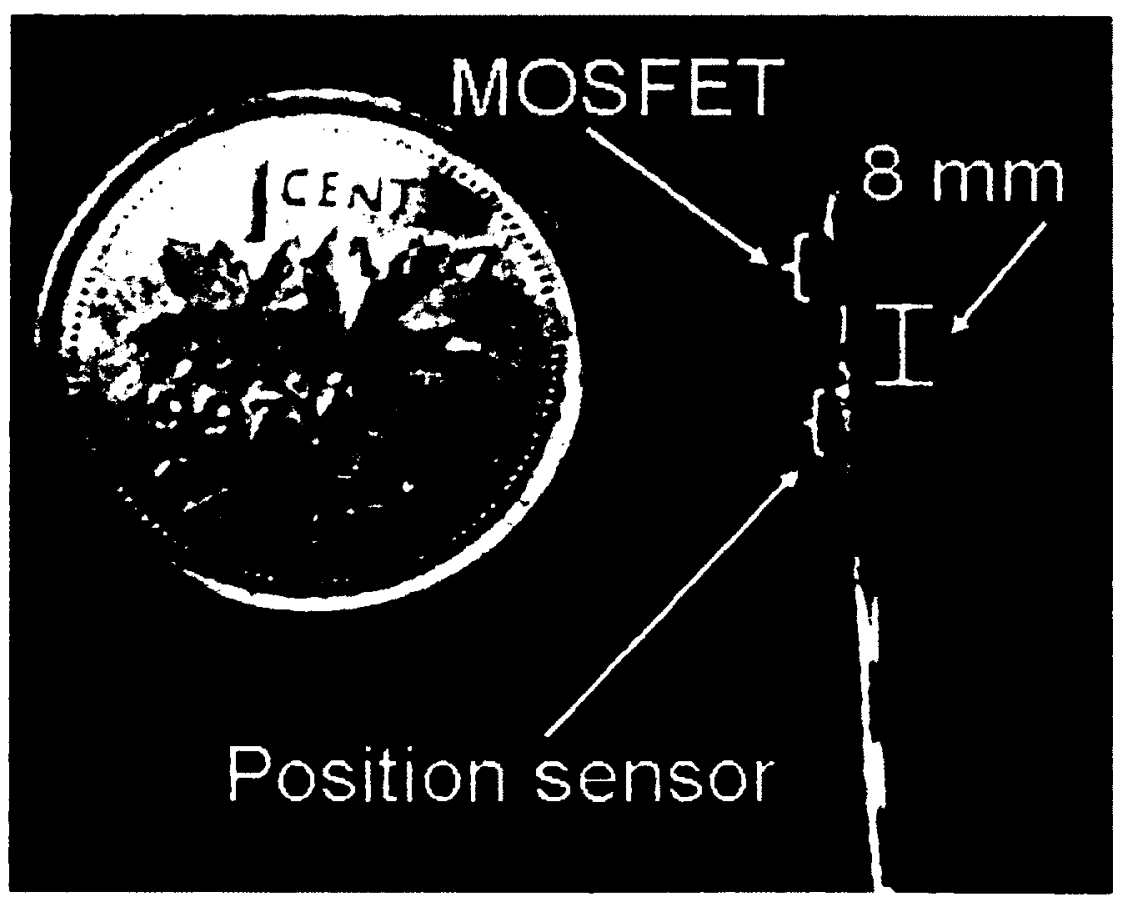

Figure 1.4 RADPOS detector. The MOSFET dosimeter is at the tip of the wire and the positioning sensor is located $8 \mathrm{~mm}$ away. Several of the detectors also have a marker at the end of the wire, above the MOSFET, that is visible on CT images. 


\subsubsection{MOSFET dosimeter}

P-channel enhancement MOSFETs consist of a negatively doped (n-type) silicon substrate, on top of which lies the source, gate and drain terminals, as seen in figure 1.5. The source and drain terminals are built on top of a region of positively-doped (p-type) silicon while the gate terminal is separated from the n-type silicon substrate by an insulating layer of silicon-dioxide. The substrate region directly below the silicon dioxide is called the channel. The voltage applied to the gate controls the conductivity of this channel. When a negative gate voltage, $V_{G}$, is applied, free electrons are repelled from the channel, leaving an electron-depleted region, and positive charge carriers are attracted from the substrate into the channel, making it more conductive. When a sufficient number of positive holes builds up, the substrate in the channel changes from n-type to ptype, creating an inversion layer which connects the source, gate and drain. The voltage required to create this inversion effect and induce a conducting channel is called the threshold voltage, $V_{t h}$. 


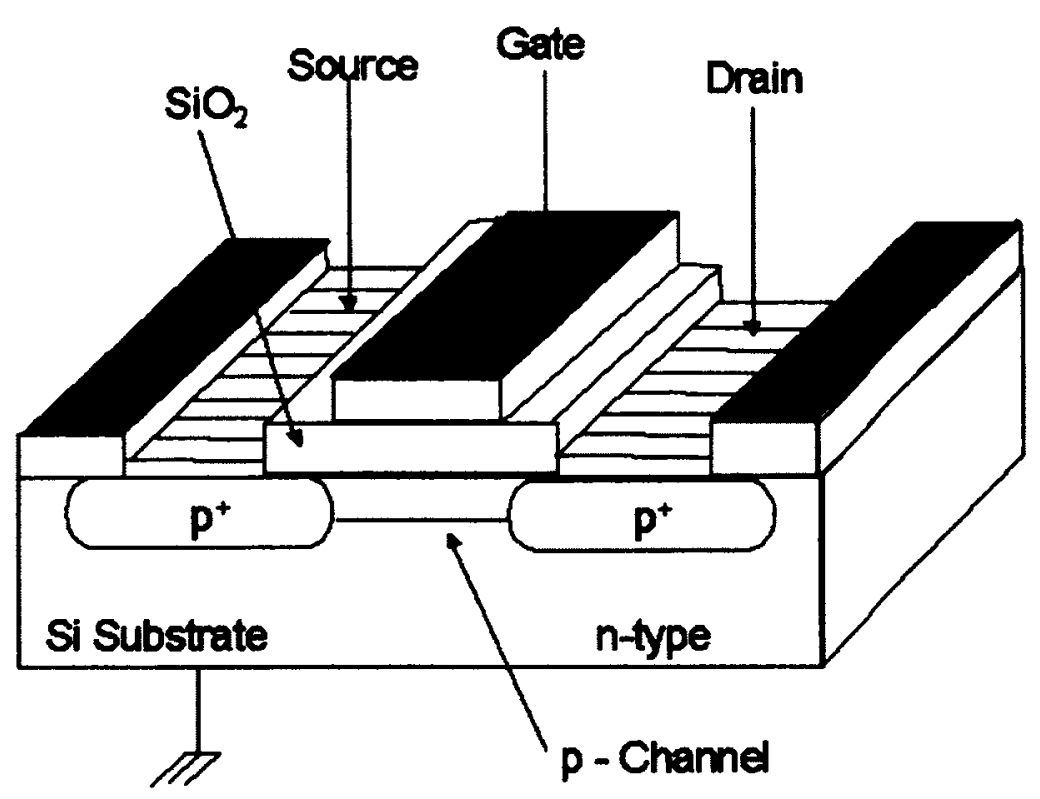

Figure 1.5 MOSFET structure. Diagram adapted from M Soubra, J Cygler, and G Mackay, "Evaluation of dual-bias-dual-metal-oxide-silicon semiconductor field effect transistor detector as radiation dosimeter," Med Phys. 21, 567-572 (1994). ${ }^{[26]}$

For use as a dosimeter, a MOSFET is bonded to a long flexible cable with approximately $1 \mathrm{~mm}$ of epoxy, which acts as a guard against contamination, moisture, and heating. When the MOSFET is exposed to ionizing radiation, electron-hole pairs are created in the insulating silicon-dioxide layer. Some of the electrons will travel to the gate and some will recombine with the holes. The holes that have not recombined with the electrons will drift to the oxide-substrate interface where some of them will become trapped. The extra interfacial charge will cause a shift in the negative voltage that must be applied between the gate and source terminals to create the conducting channel and to achieve the same current flow as before the irradiation, as seen in figure 1.6. This change in the threshold voltage, from before irradiation to after, $\Delta V_{t h}$, is proportional to the amount of dose delivered to the MOSFET. 


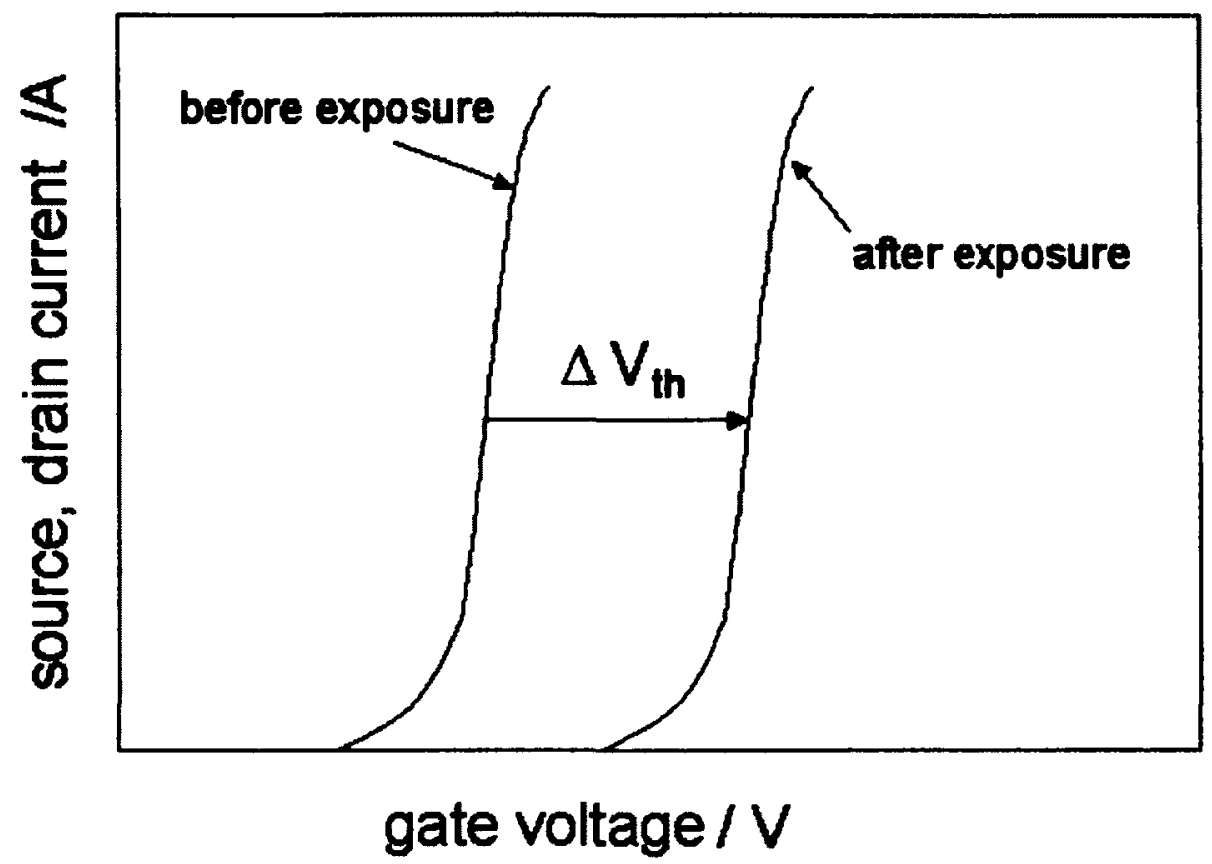

Figure 1.6 Change in threshold voltage of MOSFET with exposure to radiation.

The sensitivity of a MOSFET detector can be improved by increasing the amount of holes at the interface. This can be done by applying a positive gate bias during irradiation, which will increase the number of electrons collected at the gate, decreasing the amount of recombination and therefore increasing the number of positive holes leftover at the oxide-substrate interface. In addition, the positive gate bias pushes the holes toward the oxide interface. Another method is to increase the thickness of the oxide layer, which increases the number of electron-hole pairs created during irradiation; however, this improved sensitivity decreases the lifetime of the sensor.

The external dimensions of the MOSFETs used in the RADPOS detectors are $3.5 \times 1.0 \times 1.0 \mathrm{~mm}^{3}$, with a sensitive volume of $0.2 \times 0.2 \times 5 \times 10^{-4} \mathrm{~mm}^{3}$ for the standard sensitivity MOSFETs and of $0.2 \times 0.2 \times 1 \times 10^{-3} \mathrm{~mm}^{3}$ for the high sensitivity MOSFETs. The 
standard sensitivity MOSFETs have a nominal calibration coefficient of $1 \mathrm{cGy} / \mathrm{mV}$ while the high sensitivity MOSFETs have a nominal calibration coefficient of $0.33 \mathrm{cGy} / \mathrm{mV}$. The RADPOS probe is connected to a mobile MOSFET reader (TN-RD-16), which records the threshold voltage of the dosimeter. The software that has been created specifically for the RADPOS system allows the user to sample the dose either manually after irradiation is complete or automatically at user-defined time intervals for dynamic measurements.

\subsubsection{Positioning system}

The electromagnetic positioning sensor used with the RAPDOS system is a small cylinder $6.5 \mathrm{~mm}$ in length and $1.3 \mathrm{~mm}$ in diameter. The position coordinates of the detector are determined by monitoring the response of the sensor to a pulsed 3D magnetic field generated by a 3D-Guidance DC magnetic field transmitter. The transmitter contains three coils, each at a $90^{\circ}$ angle to the other two. When current is passed through the windings, magnetic fields with precisely-known characteristics are created along the $\mathrm{x}, \mathrm{y}$ and $\mathrm{z}$ axes. The transmitted magnetic field of each axis has a trapezoidal magnitude consisting of a short build-up period ( $250 \mu \mathrm{s})$, a steady-state period (approximately $2 \mathrm{~ms}$ ), and a fall-off period $(250 \mu \mathrm{s})$ followed by a period where the transmitter is turned off ( 2 ms). The steady-state period exists so that eddy currents in nearby metals can decay and sensor output can stabilize. This pattern is repeated sequentially for the three windings.

The transmitted magnetic field, which falls off according to the inverse cube of the distance from the transmitter $\left(1 / r^{3}\right)$, is measured by a three-axis ring core fluxgate magnetometer sensor ${ }^{[27]}$ located along the RADPOS wire. An algorithm is then used to convert the resulting values to the $x, y$, and $z$ coordinates as well as the azimuth, 
elevation and roll angles of rotation of the RADPOS probe. Similar to the dose measurements, this position information can be sampled either manually or automatically. Position coordinates can be logged with a frequency of up to $20 \mathrm{~Hz}$. Numerical data as well as graphical display are available with the software and can be viewed on the host computer.

The axes of the coordinates outputted by the RADPOS software are determined by the orientation of the transmitter, figure 1.7. For all work done in the presence of a treatment unit, the transmitter was positioned so that the $x, y$, and $z$ axes corresponded to the cross-plane, in-plane, and depth axes of the machine, respectively. This corresponds to the left/right, superior/inferior, and anterior/posterior axes of a patient, as shown in figure 1.8. The transmitter must be placed at a specific distance from the RADPOS probe to ensure the highest accuracy in the position monitoring. In this work, the transmitter was consistently placed $20 \mathrm{~cm}$ from the probe, however the extension of this optimal range has been investigated and is presented in Chapter 2.

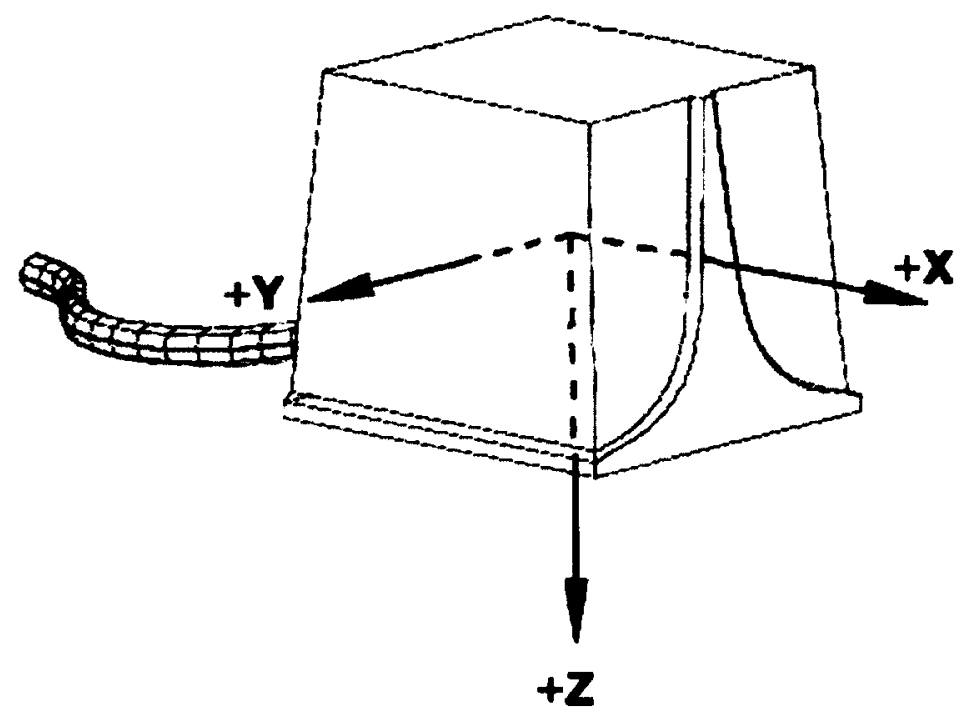

Figure 1.7 The RADPOS DC magnetic field transmitter and coordinate system. 
a)

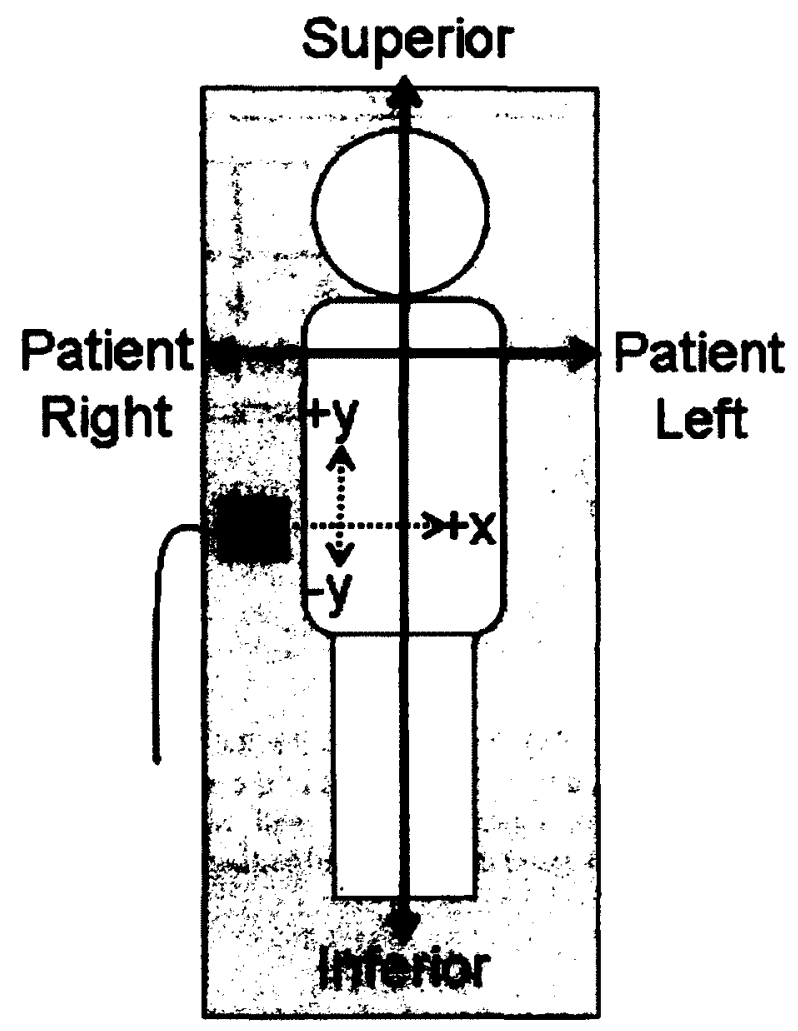

b)

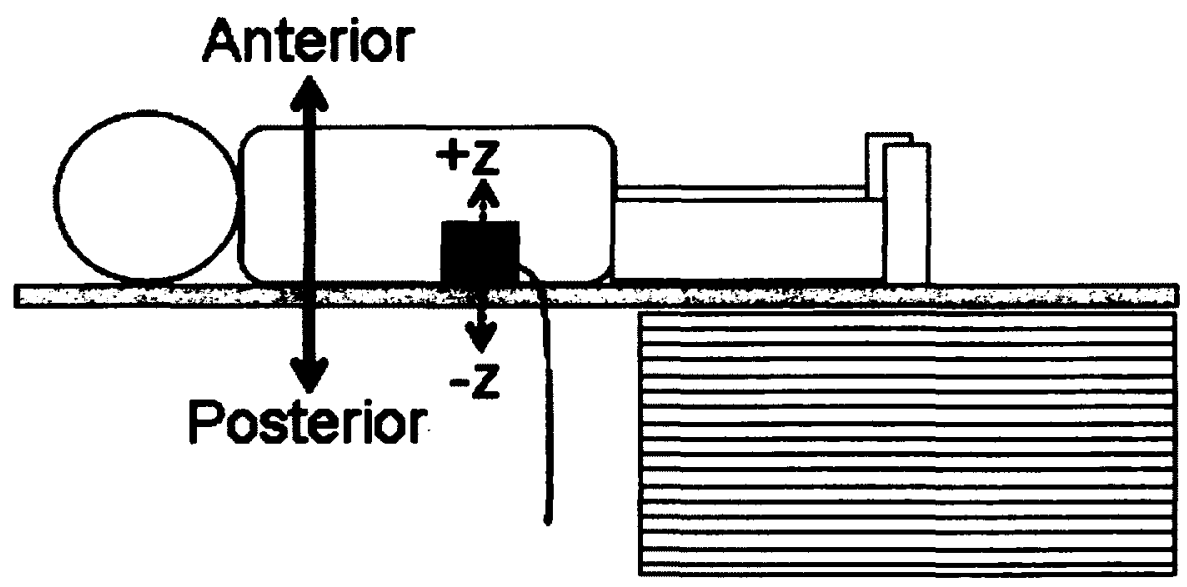


c)

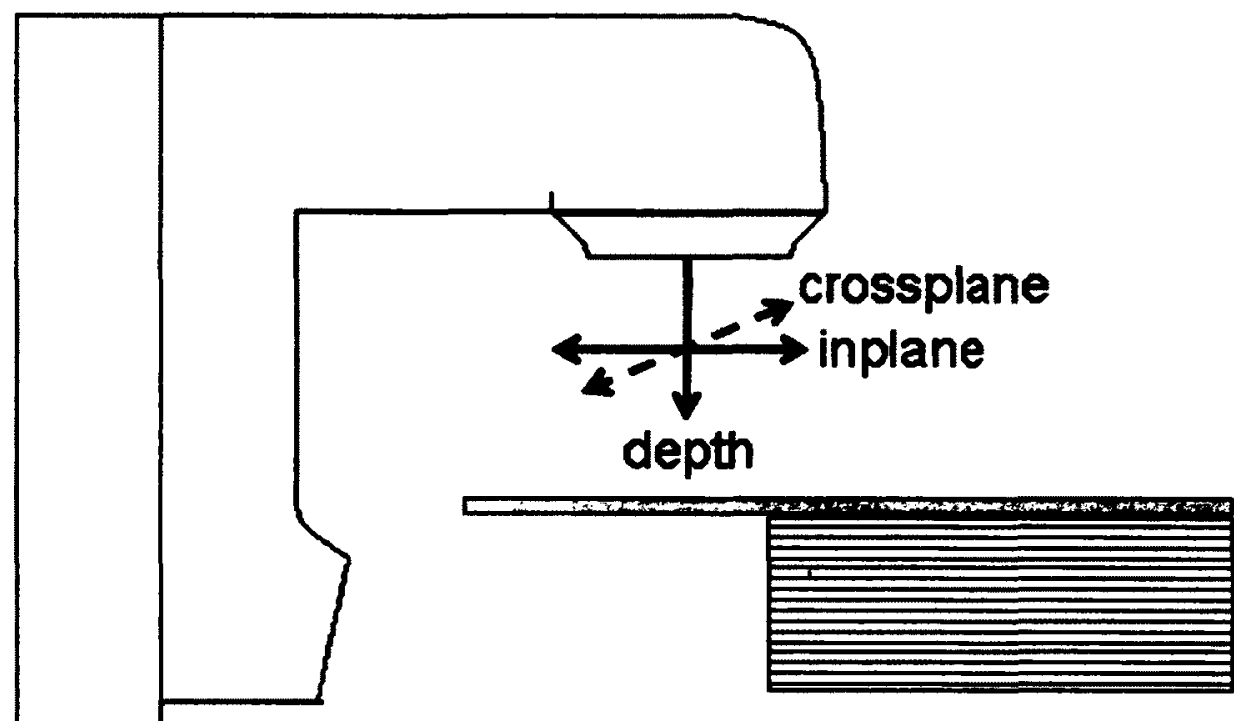

Figure 1.8 Coordinate systems of a) the patient, b) the RADPOS system, and c) the radiation beam. The RADPOS axes $(x, y, z)$ are defined by the placement of the transmitter, which has been kept consistent with respect to the patient (left/right, superior/inferior, and anterior/posterior axes) and the radiation beam (cross-plane, in-plane, and depth axes) throughout all testing. 


\subsection{THESIS OUTLINE}

This thesis covers the work done to bring a prototype of the RADPOS system from initial testing to implementation into clinical routines. Before a new tool can be used for treatment verification, the dosimetric and position accuracy must be wellcharacterized. Chapter 2 reviews preliminary tests that were carried out in the lab using both static and dynamic water-equivalent phantoms. Measurements were made with the RADPOS probe to determine any deviation from the behaviour of a regular MOSFET detector as well as to test the limitations of the position monitoring system. Once initial testing was complete, the system was taken to Montreal for a collaborative project with scientists from McGill University, as described in Chapter 3. The RADPOS system was used in conjunction with the McGill group's deformable lung phantom to simulate both free breathing and adaptive radiation treatment deliveries. The RADPOS system was used to verify delivered dose and motion of a simulated tumour while imitating a real patient treatment. After the initial testing described in Chapter 2, Health Canada granted conditional approval for use of the system on patients. Chapters 4 and 5 cover clinical trials that involved using the RADPOS system to measure patient motion and dose during external beam treatment for lung cancer (Chapter 4) and transperineal interstitial permanent prostate brachytherapy implants (Chapter 5). Aside from quantifying intraand inter-fraction variations in patient motion and dose, the feasibility of general use of the RADPOS system on patients during daily treatment fractions has also been assessed. 


\section{Chapter 2}

\section{EVALUATION OF A NOVEL 4D IN VIVO DOSIMETRY}

\section{SYSTEM}

\subsection{INTRODUCTION}

\subsubsection{In vivo dosimetry}

Radiation therapy is a complex process involving many steps, each with its own potential for error. Once the decision is made to treat a patient with radiation, a set of images is acquired and target volumes and organs at risk are contoured by a radiation oncologist. A treatment plan is then created to achieve the prescribed dose limits using various beam configurations. The treatment planning system uses complex dose calculation algorithms to calculate the total dose distribution within the patient. On each day of treatment, the patient is set-up on the treatment couch in the same way as when the images used for planning were acquired. It is becoming common practice to image the patient again while on the treatment couch to improve patient positioning accuracy. A fraction of the treatment plan is then delivered using a linear accelerator. The gantry of the machine rotates around the patient to achieve the desired beam angles and then delivers the appropriate amount of radiation in each position.

Radiation therapy departments have detailed quality assurance programs in place to address potential errors in this patient treatment process. Typical quality assurance programs involve routine tests of machine output stability, positioning lasers, treatment 
planning calculations, and data transfer methods. In vivo dosimetry is also an important part of quality assurance as it involves verification of the actual dose delivered to the patient and can identify random as well as systematic errors in treatment planning and patient set-up.

Several different types of detectors are available for in vivo dosimetry measurements including thermoluminesence dosimeters, diodes, and optically stimulated luminescence dosimeters. ${ }^{[28,29]}$ In 1994 , the first publications describing the use of MOSFETs for in vivo dosimetry appeared in literature and since then, many more publications have investigated their dosimetric properties and potential for clinical use. ${ }^{[26,}$ ${ }^{30]}$ Because of the high dose gradients present with many complex treatments, dosimeters with high spatial resolution are needed to accurately measure a region of homogeneous dose. Therefore, the small active volume of MOSFETs $(<0.5 \mathrm{~mm}$ width and $25 \mu \mathrm{m}$ thickness) minimizes the inaccuracy that can arise from having large dose gradients across the detector volume. The software used with the RADPOS system allows for realtime read-out so information regarding delivered dose can be collected while the patient is still on the treatment couch.

As mentioned above, the dosimetric properties of MOSFET detectors have been studied extensively ${ }^{[26,31-39]}$ and their performance capabilities are well known. This chapter presents preliminary testing performed using the combined MOSFETelectromagnetic positioning detector, RADPOS. Where possible, results have been compared to known capabilities of MOSFETs, when used alone, to determine any deviation in behaviour. 


\subsubsection{Positioning systems}

Patient position can vary over the course of a treatment fraction due to movement, inaccuracies in positioning, and bodily functions such as breathing and digestion. ${ }^{[3]}$ This can cause actual delivered dose to deviate from treatment plan calculations. Knowledge of patient movement can allow for monitoring of large shifts in position, as well as enable motion compensation techniques. One type of device that can be used to track patient position without requiring invasive procedures or delivering extra dose is electromagnetic positioning detectors. These systems rely on the detection of transmitted magnetic fields to calculate the position of a sensor in space. The sensors can be placed on the surface of the body or inside regions of interest to track displacements in real-time.

Several electromagnetic positioning devices exist on the market today, including the Ascension (Ascension Technology Cooperation, Burlington, Vermont), Aurora (NDI, Waterloo, Canada) and Calypso (Calypso Medical Technologies, Seattle, Washington) systems. ${ }^{[40-42]}$ The performance capabilities of these systems have been well characterized and several have been used in various radiotherapy scenarios. The positioning sensor used in the RADPOS detector is the Ascension microBird. ${ }^{[40]}$ As described in Chapter 1, this positioning system uses a pulsed DC magnetic field driven by quasi-static square wave currents. This technology is an improvement over earlier versions of Ascension detectors which used alternating current, $\mathrm{AC}$, magnetic fields. Interference from metals continues to be a problem today; however it was an even greater problem with the earlier AC-based prototypes due to eddy field noise from nearby materials. ${ }^{[40]}$ When a conductor is in the presence of time-varying magnetic fields, such as those used in early electromagnetic positioning systems, a voltage difference can be induced across the conductor. This can cause an electric current to flow within the conductor, which in turn 
creates a magnetic field of its own. This induced magnetic field, often called an eddy field, will be summed with the transmitted magnetic field of the positioning system, introducing error into the estimation of position coordinates of the sensor. The use of DCbased systems avoids this problem since measurements are made only once the magnetic field has stabilized.

Another primary source of error for electromagnetic positioning systems comes from the presence of ferromagnetic materials. When an external magnetic field is applied, a field is induced within ferromagnetic objects. This induced field can distort the transmitted steady-state magnetic field of the positioning system, affecting position coordinate calculations. The effect will be manifested as a shift in the reported position coordinates of a stationary detector when a distorting material is placed nearby. Studies have shown that electromagnetic positioning systems are more affected by materials with a high permeability, which is a measure of the extent to which a material will create a magnetic field of its own when in the presence of an external field. ${ }^{[40,42]}$

The degree of interference caused by materials commonly used in the clinic is important to understand in order to minimize noise and improve the accuracy of position measurements. The effects of various materials on the RADPOS system have been investigated and are reported in this chapter. Other characteristics of the RADPOS device have also been investigated and are compared to previously reported results from the positioning sensor when used alone as well as from other similar systems to evaluate its potential for clinical use. 


\subsection{MATERIALS AND METHODS}

\subsubsection{Dosimetric measurements}

Before the RADPOS detectors were used for dose measurements they were first calibrated in a $6 \mathrm{MV}$ linear accelerator beam. One at a time, each detector was positioned at a depth, $d_{R A D P S}$, of $5 \mathrm{~cm}$, in a solid water phantom with the MOSFET part at center of the beam and a $17.3 \mathrm{~cm}$ slab of solid water was used for backscatter. The calibrations were done at an SSD of $100 \mathrm{~cm}$. A Farmer ionization chamber, type NE 2571 (Nuclear Enterprises, UK) was positioned with its center at a depth, $d_{\text {ionchamber }}$, of $11.3 \mathrm{~cm}$ in the solid water phantom, $6.3 \mathrm{~cm}$ below the MOSFET location. One-hundred monitor units, MUs, were delivered using a $6 \mathrm{MV}$ beam and both RADPOS and ion chamber readings were taken during each trial. TG-51 protocol ${ }^{[43]}$ and percentage depth dose, PDD, curves were then used to determine the dose to water at the position of the RADPOS, $d_{\text {RADPOS }}$. A calibration coefficient, $C F$ (cGy/mV), was determined for each detector by calculating the ratio of the calculated dose delivered, $D\left(d_{R A D P O S}\right)$, to the change in threshold voltage, $\Delta V_{t h}$

$$
C F(c G y / m V)=\frac{D\left(d_{R A D P O S}\right)[c G y]}{\Delta V_{t h}[m V]}
$$

\section{Equation 2.1}

For all dosimetric measurements done in this work, the RADPOS detector was connected to the MOSFET reader at a standard bias setting of $7 \mathrm{~V}$ at least one hour before irradiations were done. An initial reading of the threshold voltage was taken and then the dose was delivered. The threshold voltage was then read again two minutes after 
the irradiation. This process was repeated three times and dose values are presented as an average of the three trials with an uncertainty equal to one standard deviation of the measurements.

\subsubsection{Dose profiles}

Dose profiles were measured in-air using an empty RFA300 water tank (Scanditronix-Wellhofer, Uppsala, Sweden) and a RADPOS with a high sensitivity MOSFET. Measurements were made in ${ }^{60} \mathrm{Co}, 6 \mathrm{MV}$, and $18 \mathrm{MV}$ beams. A water tank is a large open top cube that can be filled with water or left empty and is used for quality assurance measurements inside the treatment rooms. Two perpendicular rails inside the tank allow the user to attach a measurement probe and then move that probe in three directions within the tank using a computer control system. This set-up allowed for the RADPOS detector to be moved with respect to the beam and also provided an independent accurate reading of the position for comparison with the RADPOS readings. Once attached to the water tank positioning system, the RADPOS detector was moved to the middle of the tank, centered with respect to the beam, with the transmitter approximately $200 \mathrm{~mm}$ away along the in-plane axis. The position was zeroed and defined as the origin using both the RFA300 and the RADPOS softwares. The detector

was moved $50 \mathrm{~mm}$ in the cross-plane direction with respect to the ${ }^{60} \mathrm{Co}$ machine gantry using the computer controls of the water tank and the position as indicated by both the RADPOS software and the RFA system was recorded. The detector was then irradiated in a field size of $5 \times 5 \mathrm{~cm}^{2}$ at a source-detector-distance, SDD, of $100 \mathrm{~cm}$. In a ${ }^{60} \mathrm{Co}$ beam the detector was irradiated for $1 \mathrm{~min}\left(\Delta \mathrm{V}_{\mathrm{th}}=64 \mathrm{mV}\right.$ in the center of the beam), and in 6 and $18 \mathrm{MV}$ beams, 100 monitor units, MUs, were delivered $\left(\Delta \mathrm{V}_{\mathrm{th}}=(40-42) \mathrm{mV}\right.$ in the center 
of the beam), for each trial. This procedure was repeated between the positions $+50 \mathrm{~mm}$ and $-50 \mathrm{~mm}$, in increments of $5 \mathrm{~mm}$. The same measurements were repeated moving the RADPOS detector in the in-plane direction. The RADPOS detector was then replaced with a high quality silicon photon diode (Scanditronix, SN: DEB0102299), and the dose profiles were measured again for comparison.

\subsubsection{Angular dependence}

To test the dependence of the detector response on angular position, the RADPOS probe was positioned at a depth of $1 \mathrm{~cm}$ in a polystyrene phantom that allowed it to be rotated $360^{\circ}$ about an axis perpendicular to the beam. Since many detectors can be damaged if used in water, dry phantoms like this that both scatter and attenuate $x$-rays the same way as water, can be very useful. They are also much less cumbersome to work with than large water tanks. The RADPOS probe was originally positioned in the bubbledown orientation, which was considered as $0^{\circ}$. The detector was irradiated in a ${ }^{60} \mathrm{Co}$ beam for $1 \mathrm{~min}$ in a field of $10 \times 10 \mathrm{~cm}^{2}$ at an SSD of $80 \mathrm{~cm}\left(\Delta V_{t h}=(62-66) \mathrm{mV}\right)$. The procedure was repeated at each position for angles $0^{\circ}$ to $360^{\circ}$, in intervals of $30^{\circ}$.

\subsubsection{Field size dependence}

Previous work with MOSFET detectors has suggested a possible correlation between calibration coefficient and field size due to changes in detector sensitivity in different energy spectra and to variations in the manufacturing of individual detectors. ${ }^{[31,32,34]}$ To explore this behaviour with the RADPOS dosimeter in a high energy photon beam, two detectors, one with a high sensitivity MOSFET and one with a standard sensitivity MOSFET, were calibrated in field sizes of $6 \times 6$ to $25 \times 25 \mathrm{~cm}^{2}$. The standard sensitivity RADPOS had a change in threshold voltage of $90-108 \mathrm{mV}$ and the high sensitivity 
RADPOS had a change in threshold voltage of $235-273 \mathrm{mV}$ for these irradiations. A calibration coefficient, $C F(\mathrm{cGy} / \mathrm{mV})$, was determined for each detector and field size as described above. The procedure was repeated for field sizes up to $25 \times 25 \mathrm{~cm}^{2}$ and the calibration coefficients were normalized to the value for the $10 \times 10 \mathrm{~cm}^{2}$ field to evaluate field size dependence of the calibration coefficient.

\subsubsection{Position verification}

\subsubsection{Noise and accuracy}

The RADPOS system relies on the detection of the shape and strength of a 3D magnetic field sent out by the transmitter to correctly determine the position of the sensor. The accuracy and resolution of the position readout can therefore be affected by excessive electrical noise and background magnetic fields. Electrical noise can create magnetic fields which vary with time, and therefore interfere with the detection of the RADPOS-transmitted field by causing jumps in the recorded position. The noise and stability of the RADPOS system under optimal conditions was evaluated by securing the sensor in one position away from any external electrical devices, and taking a readout of the position coordinates every $30 \mathrm{~s}$ over a period of 60 minutes.

To test the accuracy of the position measurements, the RADPOS system was again set-up inside the empty RFA300 water tank (Scanditronix-Wellhofer, Sweden). The origin was then defined at the point $200 \mathrm{~mm}$ from the transmitter along the $\mathrm{x}$ direction, similar to figure 2.1. The detector was moved in increments of $5 \mathrm{~mm}$ using the RFA system along each of the three axes and the RADPOS measured position was recorded and compared to the RFA position after each step. Due to the geometry of the set-up, there was variation in the maximum displacement in each direction. The $\mathrm{x}$-axis scan 
ranged from $-50-330 \mathrm{~mm}$, the $y$-axis scan ranged from $0-365 \mathrm{~mm}$, and the scan along the z-axis ranged from $0-300 \mathrm{~mm}$. This set-up was chosen to maximize the distance tested, and results were assumed to be symmetric. The scans were done in one direction, rather then symmetrically, to maximize the distance tested. The orientation of the transmitter and the direction of the scan in each direction is shown in figure 2.1.

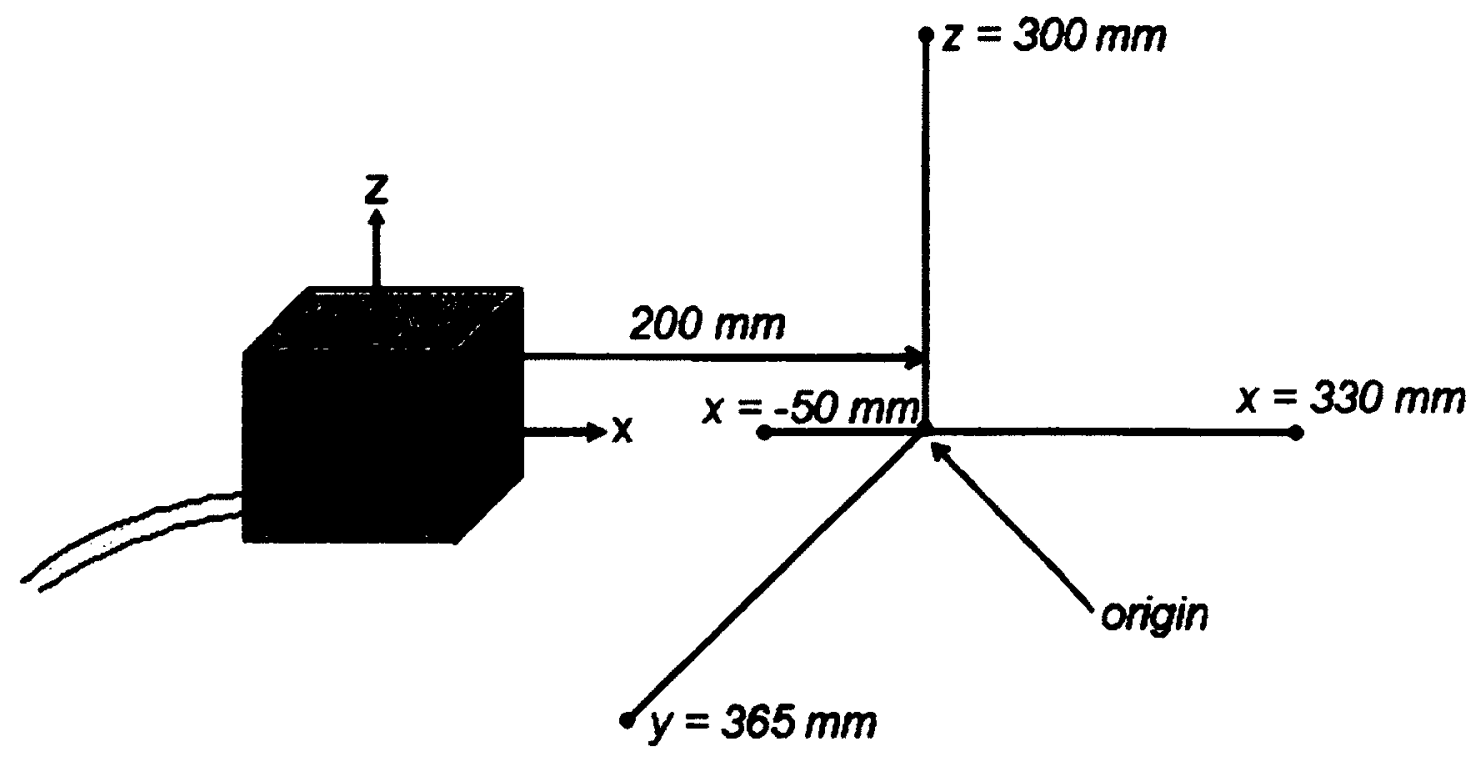

Figure 2.1. Direction of scans in the $x, y$, and $z$ directions. The origin was defined as a point $200 \mathrm{~mm}$ away from the transmitter, along the $x$ direction.

\subsubsection{Interference}

The presence of magnetic and/or electrically conductive materials can cause a systematic shift in the measured position by distorting the RADPOS-transmitted field. The magnitude of the distortion caused by a material can depend on the ferromagnetic property, the electrical conductivity and the physical shape of the material sample. To study the effect of metals and other materials on the RADPOS signal, square samples approximately $6 \mathrm{~mm}$ in thickness of non magnetic stainless steel, aluminium, lead, brass, 
acrylic, lexan and polystyrene, were used. The samples were of sizes $15 \times 15,20 \times 20$ and $25 \times 25 \mathrm{~cm}^{2}$ for each type of material. The choice of samples studied for interference was dictated by materials encountered in the treatment and simulation rooms. First, the detector was secured on a stationary slab of solid water, $200 \mathrm{~mm}$ from the transmitter, along the $\mathrm{x}$ axis. The initial RADPOS position was recorded and zeroed, and then a sample of one of the materials was positioned vertically at distance $a=200 \mathrm{~mm}$ away from the transmitter along the $\mathrm{x}$ axis, as seen in figure 2.2

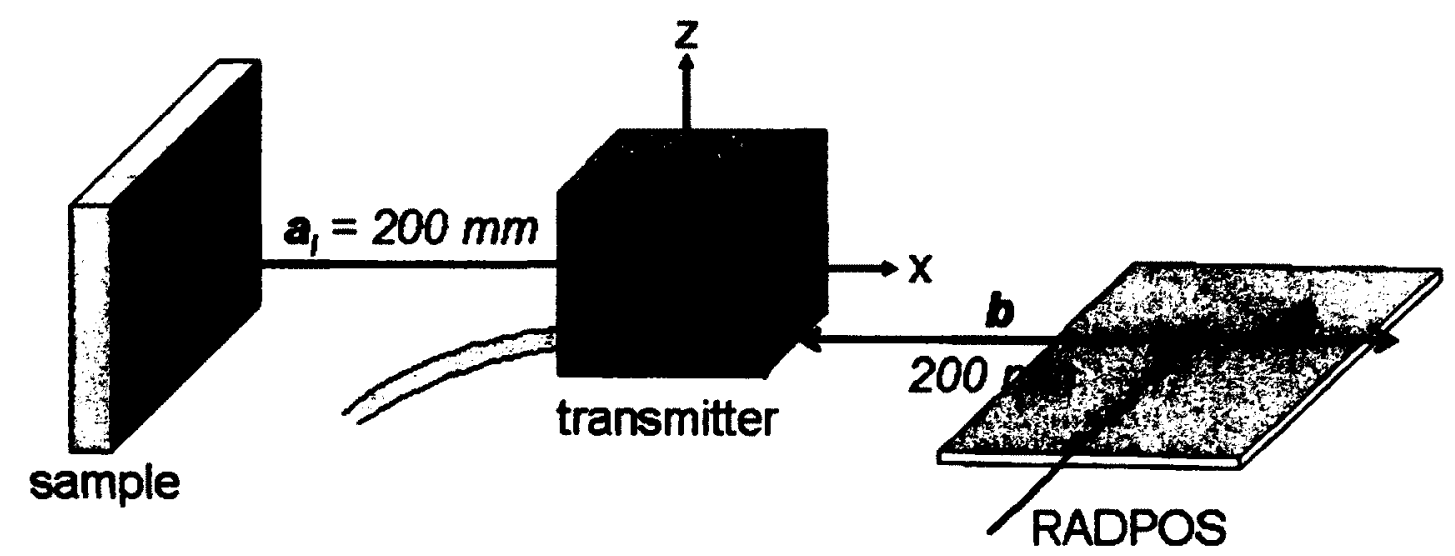

Figure 2.2. Set-up for material interference tests.

The RADPOS position was recorded again with the sample present. This procedure was repeated for each sample at distances ranging from $a=200$ to $10 \mathrm{~mm}$. The effect of each sample material was then tested when placed between the transmitter and detector, at various points along line $b$. Finally, each sample was positioned at distances ranging from 10 to $200 \mathrm{~mm}$ away from the detector, along line $c$. At each position, the positional error was calculated as a resultant vector length, $r_{\text {error }}$, of the $x, y$, and $z$ coordinates. 


\subsubsection{Preliminary clinical evaluation}

Before the RADPOS system was used to study respiratory motion in patients during treatment, a dynamic phantom was used to simulate such measurements in a controlled lab setting. This investigation evaluated the potential of using RADPOS detectors to record external surface motion before integrating such measurements into patients' daily treatments. A Quasar Respiratory Motion Phantom ${ }^{\mathrm{TM}}$ (Modus Medical Devices Inc, London, $\mathrm{ON}$, Canada) was used to simulate patient movement motion due to breathing in a constant and controlled manner and this motion was measured by the detector. One RADPOS probe was secured inside a translation stage which was placed inside the phantom and two more detectors were attached to the back of the stage. This stage can be moved by a motor in an oscillating motion along the superior/inferior (horizontal) direction with a user-defined frequency and amplitude, see figure 2.3. The phantom also has a chest wall platform that moves in the anterior/posterior (vertical) direction. The amplitude of this platform is approximately one quarter of the motion of the translation stage. The user also has more control over the amplitude of the translation stage, so for these two reasons initial testing involved using the translation stage. Once the RADPOS detectors were in place, the phantom was set to move the stage with an amplitude of $40 \mathrm{~mm}$ with frequencies ranging from 8 to 20 cycles per minute and the motion was measured by the RADPOS detectors throughout several breathing cycles. For these trials, the RADPOS system was used in "automatic logging" mode. This allows the user to pick a defined time interval, as small as $100 \mathrm{~ms}$, after which the position will continually be recorded or to use a "minimum time interval" option, which acquires data 
as quickly as possible, depending on the computer speed. The "minimum time interval" option was used here for the automatic logging.

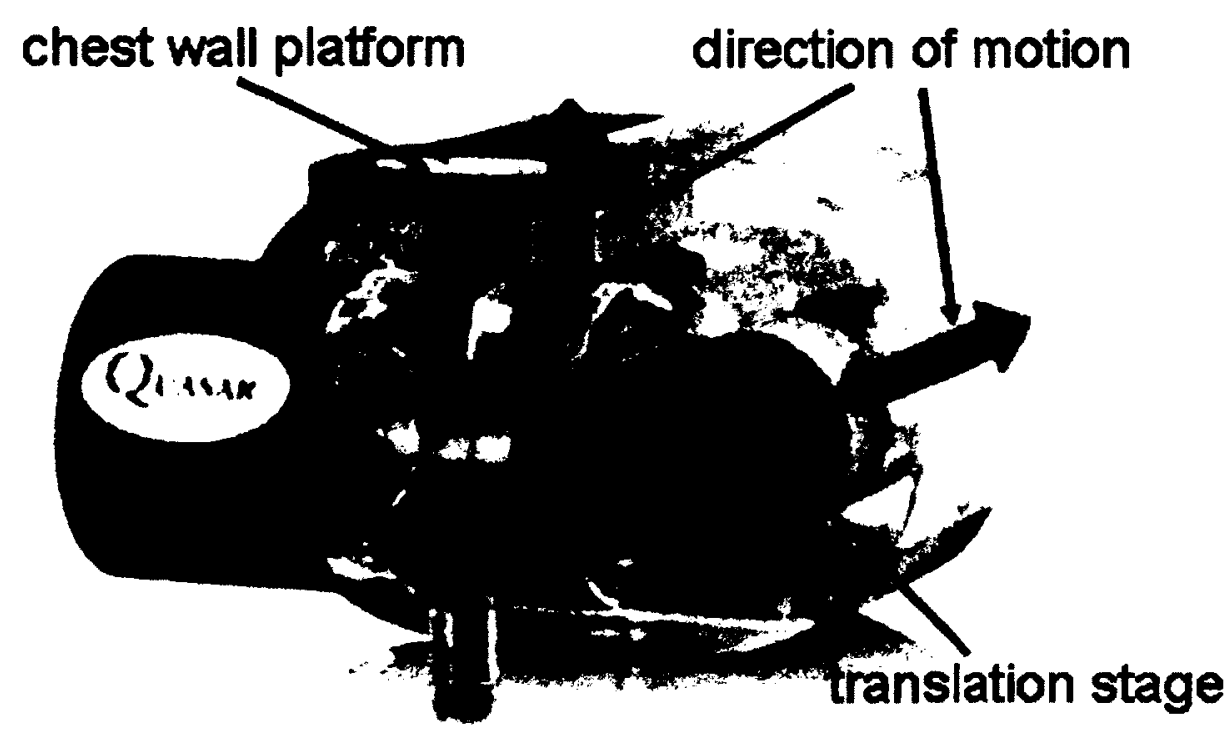

Figure 2.3. Quasar Respiratory Motion Phantom ${ }^{\mathrm{TM}}$. The phantom has two moving components, the translation stage and the chest wall platform. The translation stage moves horizontally, and has been always set-up to move in the superior/inferior direction for this work.

\subsection{RESULTS \& DISCUSSION}

\subsubsection{Dosimetric measurements}

\subsubsection{Dose profiles}

A comparison of the dose profiles measured with the RADPOS system and with the high quality Scanditronix silicon photon diode is shown in figure 2.4. MOSFET dosimeters have a reproducibility of $3 \%^{[37-39]}$, which is similar to the response seen in this work when the MOSFET is combined with the positioning sensor into one RADPOS probe. Over the entire profile curve, the average absolute deviation between the RADPOS and diode relative dose measurements was $5.3,3.1$, and $2.9 \%$ of the maximum 
dose on the central axis for the ${ }^{60} \mathrm{Co}, 6 \mathrm{MV}$, and $18 \mathrm{MV}$ beams, respectively. The relative response of the RADPOS probe is less than the diode in lower energy spectrum present outside the beam as the RADPOS measurements are slightly lower than the diode measurements in the penumbra region for the ${ }^{60} \mathrm{Co}$ beam, as seen in figure 2.4. This is due to the difference in atomic number between the silicon diode and the silicon dioxide, $\mathrm{SiO}_{2}$, of the MOSFET dosimeter in the RADPOS. Silicon has an atomic number of 14, while the effective atomic number of $\mathrm{SiO}_{2}$ is approximately 10 . At lower photon energies, such as those present outside the field, the photoelectric effect becomes important, with an atomic cross section proportional to $\mathrm{Z}^{3.8}$ for low $\mathrm{Z}$ materials. Since the silicon diode has a larger atomic number, it will have more interactions than the silicon dioxide in the RADPOS, leading to the noted over-response. ${ }^{[44]}$ This trend of the higher diode response outside of the radiation field was not as apparent in the higher energy beams, which is in general agreement with the findings of other groups for selectively shielded silicon diodes. ${ }^{[45]}$ Similar results were found for the in-plane dose profiles. 
a)

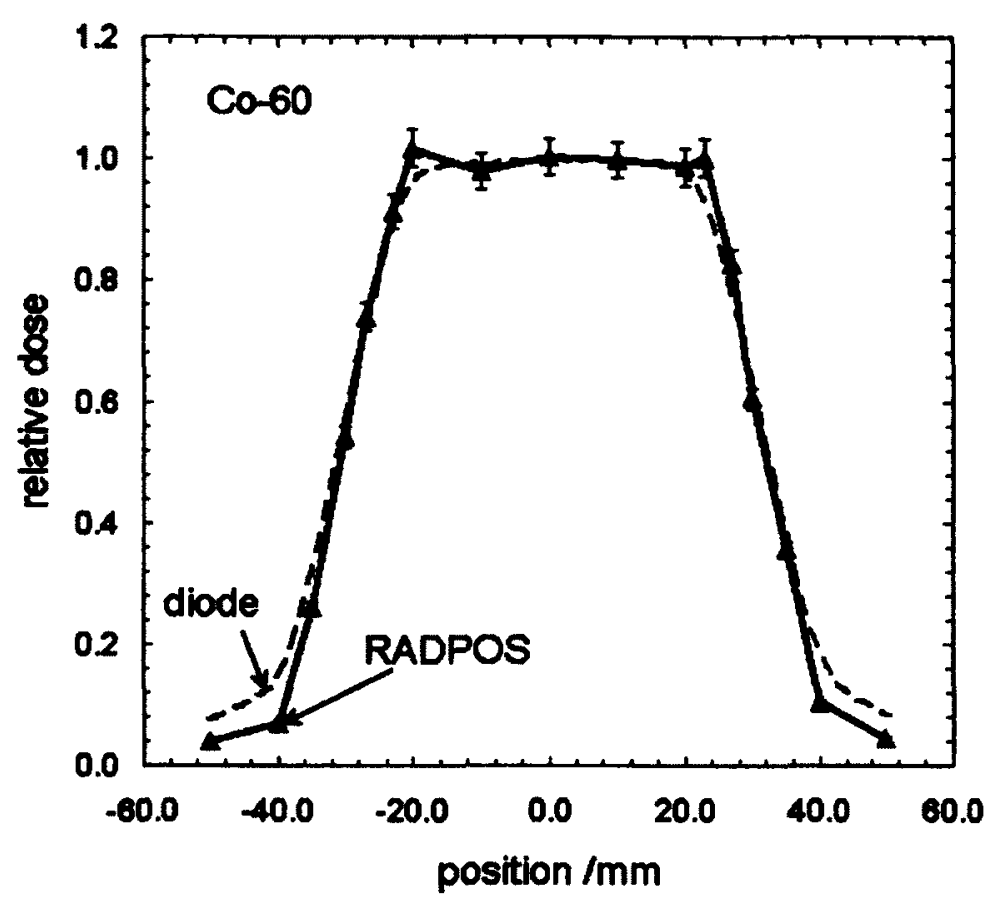

b)

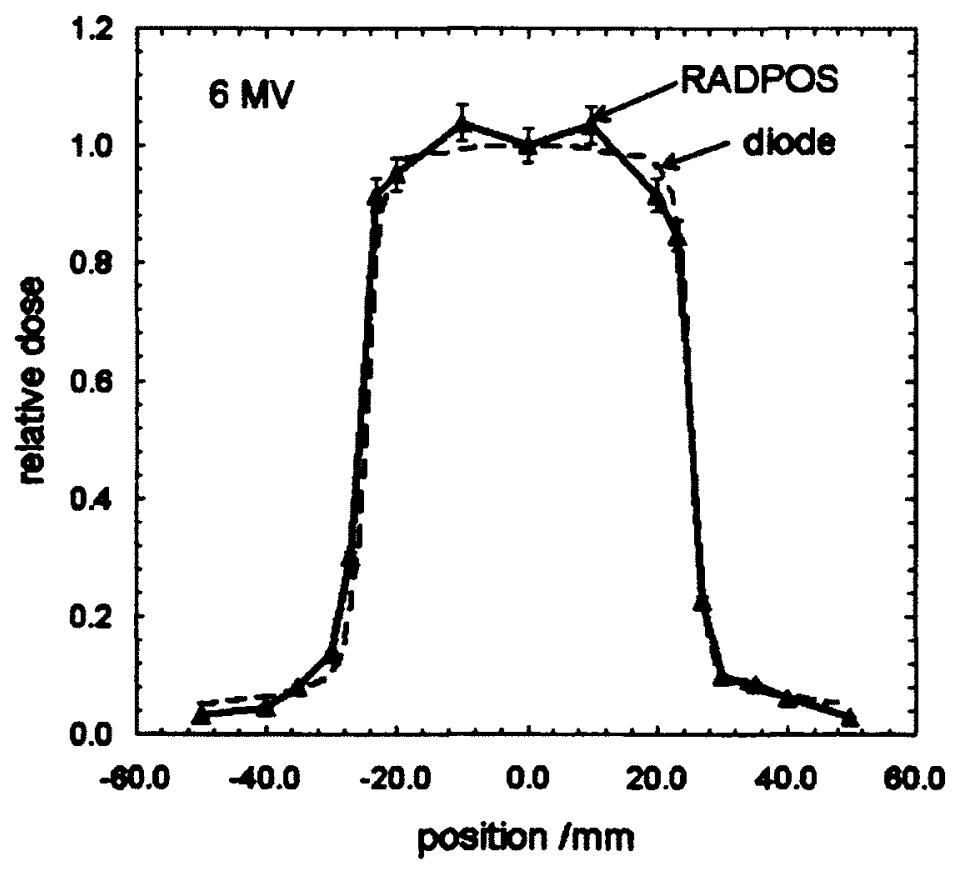


c)

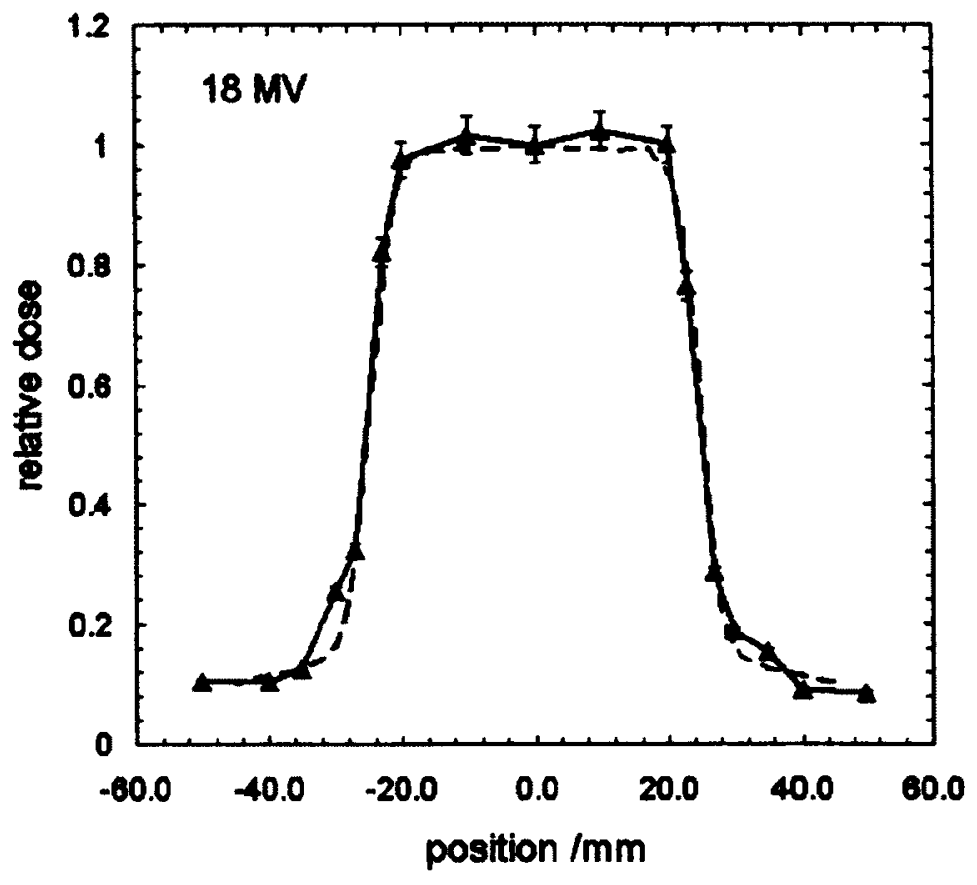

Figure 2.4. In-air cross-plane dose profiles measured with the RADPOS prototype and the RFA300 and diode for a) $\left.{ }^{\circ} \mathrm{Co} \mathrm{b}\right) 6 \mathrm{MV}$ and c) $18 \mathrm{MV}$ beams. The dashed line in each figure represents diode measurements and the solid line represents RADPOS measurements. Error bars represent the standard deviation of the data for 3 measurements. The detector was positioned at an SDD of $100 \mathrm{~cm}$.

\subsubsection{Angular dependence}

Measurements of the angular response of the RADPOS probe over $360^{\circ}$ range in a ${ }^{60} \mathrm{Co}$ beam are shown in figure 2.5 . The average angular response of the probe over $360^{\circ}$ ranged from $-2.6 \%$ to $+3.6 \%$. The directional dependence of microMOSFETs, when used alone, is reported as $\pm 3 \%$ for beam energies $75 \mathrm{kV}$ to $18 \mathrm{MV}$ when measured under full build-up conditions. ${ }^{[46]}$ Therefore, the addition of the positioning sensor slightly increased the variation of detector response as a function of its orientation. This may be due to beam attenuation and/or additional scatter from the positioning sensor towards the MOSFET. 


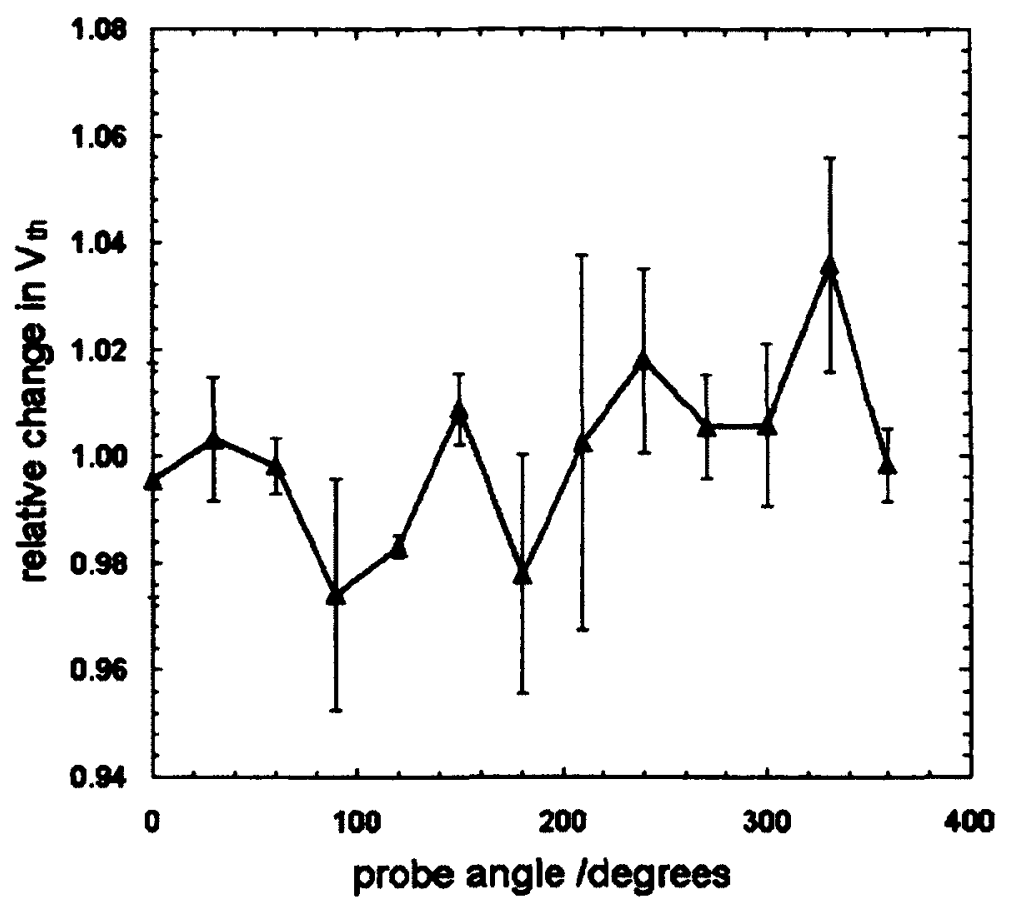

Figure 2.5. Relative change in the threshold voltage, $\triangle \mathrm{Vth}$, of the RADPOS prototype plotted as a function of probe angle (bubble down $=0^{9}$ ). Energy: ${ }^{60} \mathrm{Co}$, field size: $10 \times 10 \mathrm{~cm}^{2}$, polystyrene phantom, $S S D=80 \mathrm{~cm}$, depth $1 \mathrm{~cm}$.

\subsubsection{Field size dependence}

The standard sensitivity detector showed a significant decrease in calibration coefficient with increased field size, as seen in figure 2.6. The maximum deviation in the measured calibration coefficient was $-4.6 \%$ for field sizes larger than $10 \times 10 \mathrm{~cm}^{2}$, and $2.5 \%$ for field sizes smaller than $10 \times 10 \mathrm{~cm}^{2}$ for this detector. The high sensitivity detector did not show as much of a decrease in calibration coefficient for the large field sizes, with maximum deviation of $-0.6 \%$. There was however an increase of $2.3 \%$ in the calibration coefficient for the smaller field sizes. Other work ${ }^{[23]}$ has shown a stronger dependence on field size for high sensitivity MOSFETs, which suggests that this behaviour is dependent 
on the manufacturing of the individual detectors and therefore no general conclusions can be made.

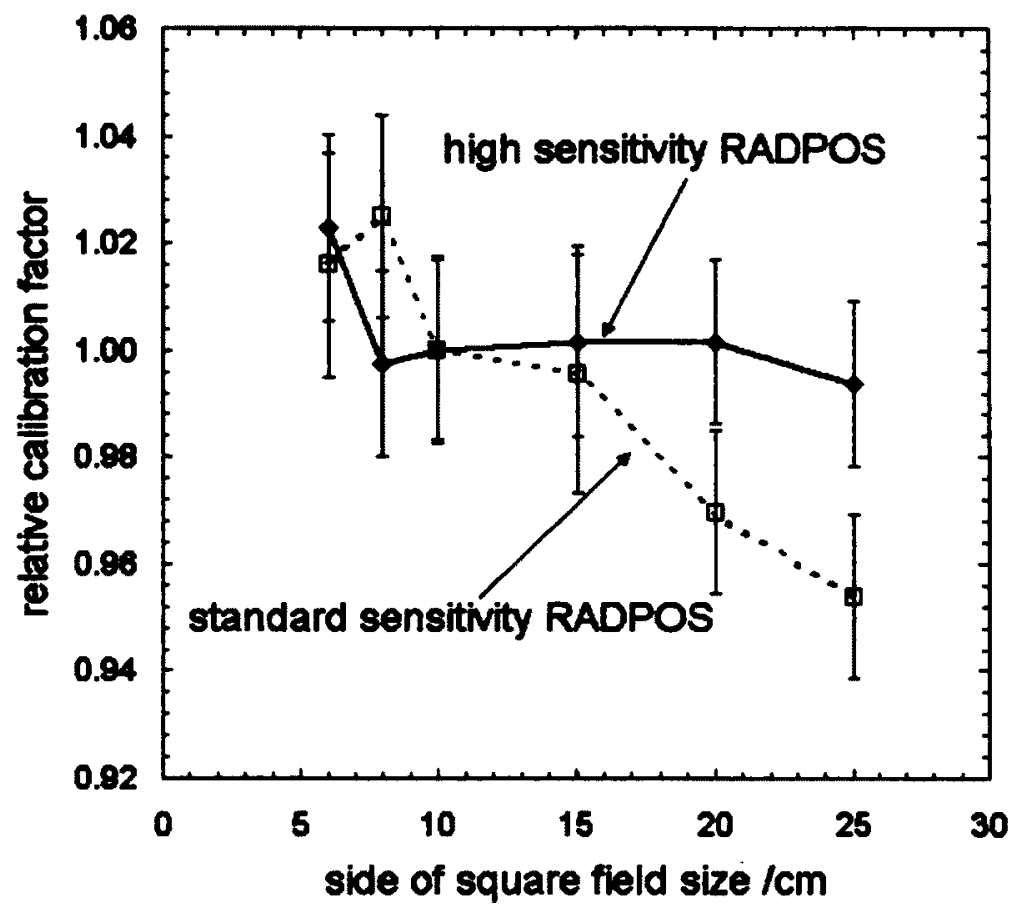

Figure 2.6. Relative calibration coefficients measured at field sizes of $6 \times 6 \mathrm{~cm}^{2}$ to $25 \times 25 \mathrm{~cm}^{2}$ for standard and high sensitivity MOSFETs.

\subsubsection{Position verification}

\subsubsection{Noise and accuracy}

Noise was calculated using the difference between the root mean squared RADPOS position and the initial position, which was $(0,0,0)$. Since the detector was stationary through the measurement period, all recorded positions should be zero. Any deviations from this value will represent system noise. Over a period of one hour, the measured noise was within $\pm 0.11 \mathrm{~mm}$. The position remained stable, as no significant drift in any direction was observed during this time. Similar results were also found when 
the system was set-up on a treatment couch and the radiation beam was turned on. Results from the verification of the accuracy of the RADPOS position are displayed in figure $2.7 \mathbf{a}$.

This figure shows the deviation of the RADPOS measured positions from the actual positions, as determined by the RFA system, as a function of the distance from the transmitter. In the $x$ (in-plane) and $y$ (cross-plane) directions, an optimal range of the probe from the transmitter can be seen as the deviations slightly increased up to a point, returned to zero, and then rapidly increased in magnitude. This point was $470 \mathrm{~mm}$ in the $\mathrm{x}$ direction and $215 \mathrm{~mm}$ in the $\mathrm{y}$ direction. In the $\mathrm{x}$ direction, the average absolute deviation within the range $150-470 \mathrm{~mm}$ was $(1.1 \pm 0.7) \mathrm{mm}$ with a maximum deviation of $2.5 \mathrm{~mm}$. Since the origin was defined as a point $200 \mathrm{~mm}$ away from the transmitter along the $\mathrm{x}$ direction, the range 150 to $470 \mathrm{~mm}$ corresponds to a distance from the origin of -50 to $270 \mathrm{~mm}$ from the origin, as shown in figure 2.7 . In the $y$ direction, the average absolute deviation within the range 0 to $215 \mathrm{~mm}$ was $(1.3 \pm 0.8) \mathrm{mm}$, with a maximum deviation of $2.5 \mathrm{~mm}$. Along the $\mathrm{z}$ (depth) axis the accuracy initially decreased linearly up to approximately $200 \mathrm{~mm}$, with a maximum deviation of $4.7 \mathrm{~mm}$, and then fell much more rapidly. To achieve accuracy similar to that in the optimal range defined in the $x$ and $y$ directions, the detector should remain within $100 \mathrm{~mm}$ of the transmitter in the depth direction. In this range, 0 to $100 \mathrm{~mm}$, the average absolute deviation is $(1.1 \pm 0.4) \mathrm{mm}$, with a maximum deviation of $1.7 \mathrm{~mm}$. These values remained consistent with large accumulated dose received by the probe, which means no significant radiation damage occurred to the positioning sensor. The optimal volume for RADPOS position measurements, defined by the coordinates explained above, is shown in figure $2.7 \mathrm{~b}$. 
Within this volume, $3 D$ RADPOS displacements have an uncertainty of $\sigma_{\mathrm{RADPOS} \_3 \mathrm{D}}=2$ $\mathrm{mm}$ as shown in equation 2.2 .

Earlier studies on other electromagnetic positioning systems have concluded that there is a more linear trend in position accuracy with distance from transmitter than what was seen here with the RADPOS detector. Results from other groups on optimal range from transmitter and expected uncertainties within the region for various systems are shown in table 2.1. ${ }^{[27,40-42]}$ Some groups define a specific range along each of the three axes, while others only specify an root-mean squared distance, $r$. When available, the root-mean squared positional error within the region, $\sigma_{3 \mathrm{D}}$ is given, otherwise the onedimensional error, $\sigma_{1 D}$, is shown. Testing on the Ascension system has shown root-mean squared positional errors ranging from -0.5 to $3.2 \mathrm{~mm}$ depending on distance from transmitter, while for the Aurora system, the root-mean squared positional error can range from 0.2 to $5.4 \mathrm{~mm}$ for distances up to $600 \mathrm{~mm} \cdot{ }^{[27,42]}$ Another type of electromagnetic localization system from Calypso Medical Technologies has also been extensively tested for position accuracy. ${ }^{[41]}$ The uncertainties in position measurements taken by this system range from -0.35 to $0.04 \mathrm{~mm}$ for the individual coordinates for transmitter distances of $80-270 \mathrm{~mm} .^{[41]}$ The uncertainties quoted in all of these studies depend on the optimal volume as defined by the user. Different applications may require smaller or larger sensor distances with respect to the transmitter, so the user could re-evaluate the uncertainty within the expected region of motion to have a more accurate representation of expected uncertainties. 
a)

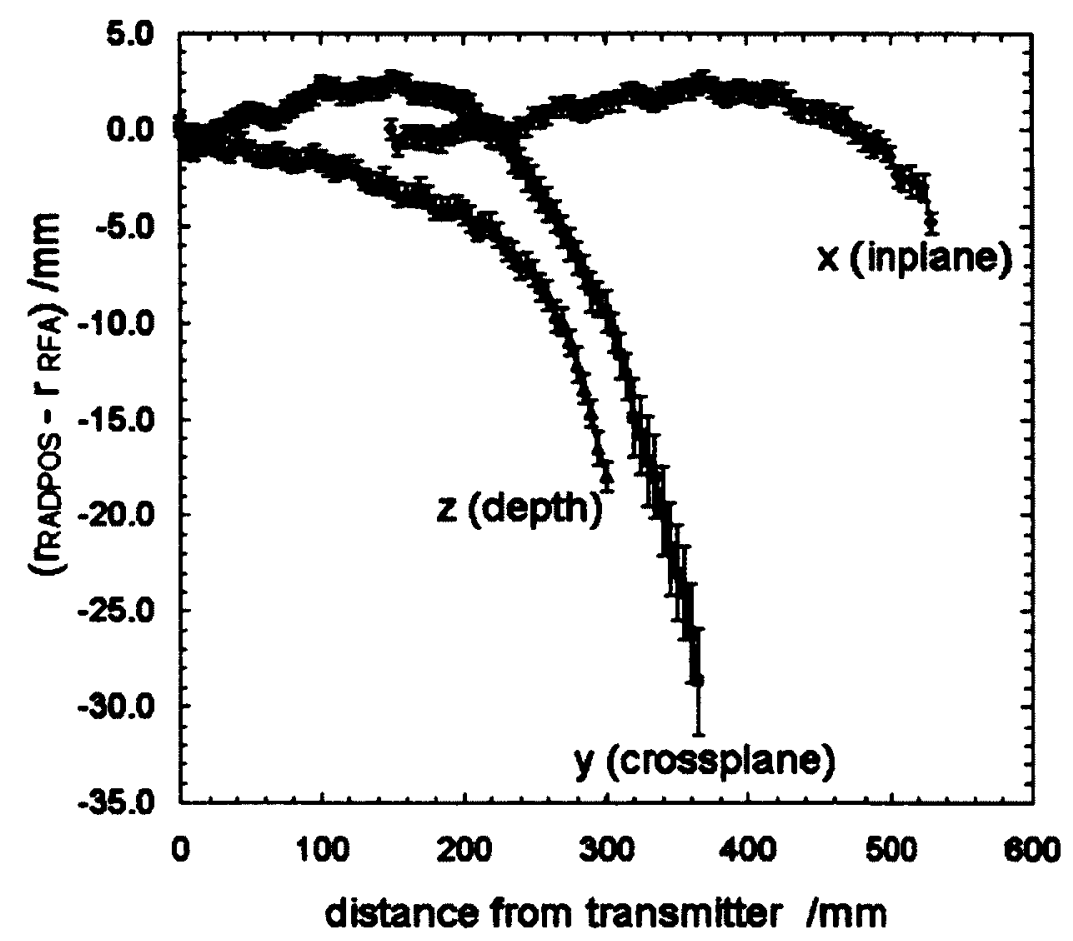

b)

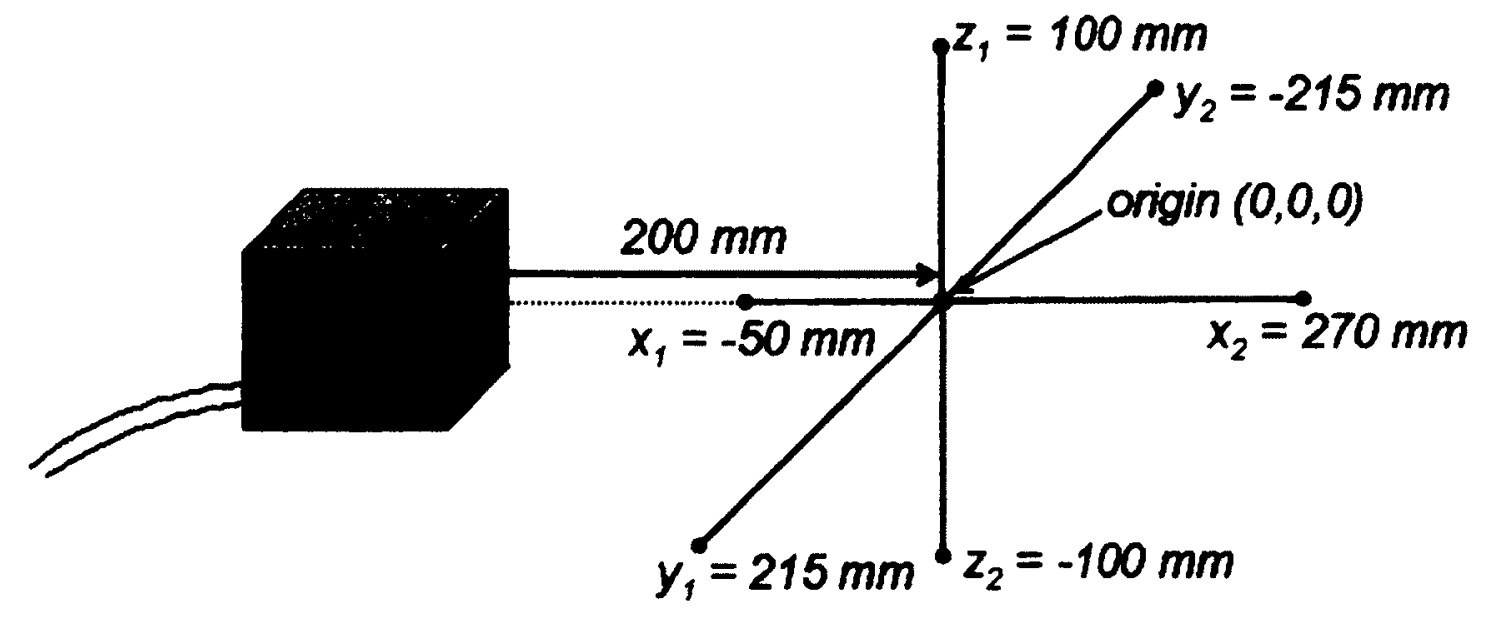

Figure 2.7. a) Difference between RADPOS and RFA positions taken along the $x$ (in-plane) axis, $y$ (crossplane) axis and $z$ (depth) axis. b) Boundaries for optimal volume for RADPOS position measurements based on data shown in part a of the figure. With the origin defined at a point $200 \mathrm{~mm}$ away from the transmitter along the $x$ axis, these boundaries extend from $x=-50$ to $320 \mathrm{~mm}, y=-215$ to $215 \mathrm{~mm}$, and $z=$ -100 to $100 \mathrm{~mm}$. 


$$
\begin{aligned}
& \sigma_{R_{1 D P O S_{-} 3 D}}=\left\lfloor\sqrt{\left(1.1^{2}\right)+\left(1.3^{2}\right)+\left(1.1^{2}\right)}\right\rfloor_{\mathrm{mm}} \\
& \sigma_{\text {RADPOS } 3 D_{-}}=2 \mathrm{~mm}
\end{aligned}
$$

Equation 2.2

Table 2.1 Summary of published results on optimal range and uncertainty for various electromagnetic

\begin{tabular}{|c|c|c|c|}
\hline Stady & Syctem & $\begin{array}{l}\text { Optimal distance } \\
\text { trow tranisumer }\end{array}$ & $\begin{array}{l}\text { Uneartalinty } \\
\text { whing eptimal } \\
\text { voluge }\end{array}$ \\
\hline $\begin{array}{l}\text { Cherpak et al., Med } \\
\text { Phys } 2009 \text { (results } \\
\text { presented in this thesis) }\end{array}$ & $\begin{array}{l}\text { RADPOS } \\
\text { (Ascension } \\
\text { microBird) }\end{array}$ & $\begin{array}{l}x=150 \text { to } 470 \mathrm{~mm} \\
y=-215 \text { to } 215 \mathrm{~mm} \\
z=-100 \text { to } 100 \mathrm{~mm}\end{array}$ & $\begin{array}{c}\sigma_{1 \mathrm{D}}=1.1-1.3 \mathrm{~mm} \\
\sigma_{3 \mathrm{D}}=2 \mathrm{~mm}\end{array}$ \\
\hline $\begin{array}{l}\text { Manufacturer } \\
\text { specifications } \\
\text { (Ascension Technology } \\
\text { Cooperation) } \\
\end{array}$ & $\begin{array}{l}\text { Ascension } \\
\text { microBird }\end{array}$ & $\begin{array}{l}x=200 \text { to } 360 \mathrm{~mm} \\
y=-200 \text { to } 200 \mathrm{~mm} \\
z=-100 \text { to } 100 \mathrm{~mm}\end{array}$ & $\sigma_{3 \mathrm{D}}=1.4 \mathrm{~mm}$ \\
\hline $\begin{array}{l}\text { Schneider and Stevens, } \\
\text { Proceedings of SPIE } \\
\text { Medical Imaging } 2007\end{array}$ & $\begin{array}{l}\text { Ascension } \\
\text { microBird }\end{array}$ & $\begin{array}{l}x=200-500 \mathrm{~mm} \\
y=-100-100 \mathrm{~mm} \\
z=-150-150 \mathrm{~mm}\end{array}$ & $\sigma_{3 \mathrm{D}}=1.3 \mathrm{~mm}$ \\
\hline $\begin{array}{l}\text { Hummel et al., Med } \\
\text { Phys } 2005\end{array}$ & $\begin{array}{l}\text { Ascension } \\
\text { microBird }\end{array}$ & $r=200 \mathrm{~mm}$ & $\begin{array}{c}\sigma_{3 \mathrm{D}}=<3.2 \mathrm{~mm} \\
(\text { for } \mathrm{r}<300 \mathrm{~mm} \text { ) }\end{array}$ \\
\hline $\begin{array}{l}\text { Milne et al., } \\
\text { J. Biotech } 1996\end{array}$ & $\begin{array}{l}\text { Ascension } \\
\text { Flock of Birds }\end{array}$ & $r=225$ to $640 \mathrm{~mm}$ & $\begin{array}{c}\sigma_{3 \mathrm{D}}=0.5-1.0 \mathrm{~mm} \\
(1.8 \% \text { of step } \\
\text { size })\end{array}$ \\
\hline $\begin{array}{l}\text { Hummel et al., Med } \\
\text { Phys } 2005\end{array}$ & NDI Aurora & $\begin{array}{l}\text { error proportional } \\
\text { to distance }\end{array}$ & $\begin{array}{c}\sigma_{3 \mathrm{D}}=<5.4 \mathrm{~mm} \\
(\text { for } \mathrm{r}<300 \mathrm{~mm})\end{array}$ \\
\hline $\begin{array}{l}\text { Hummel et al., Med } \\
\text { Phys } 2002\end{array}$ & NDI Aurora & $\begin{array}{l}x=0-400 \mathrm{~mm}(1) \\
x=0-600 \mathrm{~mm}(2)\end{array}$ & $\begin{array}{l}\sigma_{3 \mathrm{D}}=0.2 \mathrm{~mm}(1) \\
\sigma_{3 \mathrm{D}}=1.0 \mathrm{~mm}(2)\end{array}$ \\
\hline $\begin{array}{l}\text { Balter et al., } \\
\text { Int J Radiat Oncol Biol } \\
\text { Phys } 2005\end{array}$ & Calypso & $x=80$ to $270 \mathrm{~mm}$ & $\sigma_{1 D}=-0.35-0.04$ \\
\hline
\end{tabular}
positioning systems. ${ }^{[27,40-12,47]}$ 


\subsubsection{Interference}

The presence of polystyrene, lexan and acrylic caused no measureable effect on the perceived position of the RADPOS detector for all sample sizes. Aluminium, lead, and brass caused shifts of varying degrees in the perceived position depending on the size of the material and its proximity to the system. The positional error was greatest when the samples were placed between the detector and the transmitter, along line $b$, figure 2.2 , closest to the transmitter. Compared to the other materials, aluminium caused the greatest error, distorting the position by $154.2 \mathrm{~mm}$ when the $25 \times 25 \mathrm{~cm}^{2}$ sample was placed within $20 \mathrm{~mm}$ of the transmitter, along line $b$. Figure 2.8 shows results for the $25 \times 25 \mathrm{~cm}^{2}$ samples of the interfering materials along line $c$, at various points away from the detector.

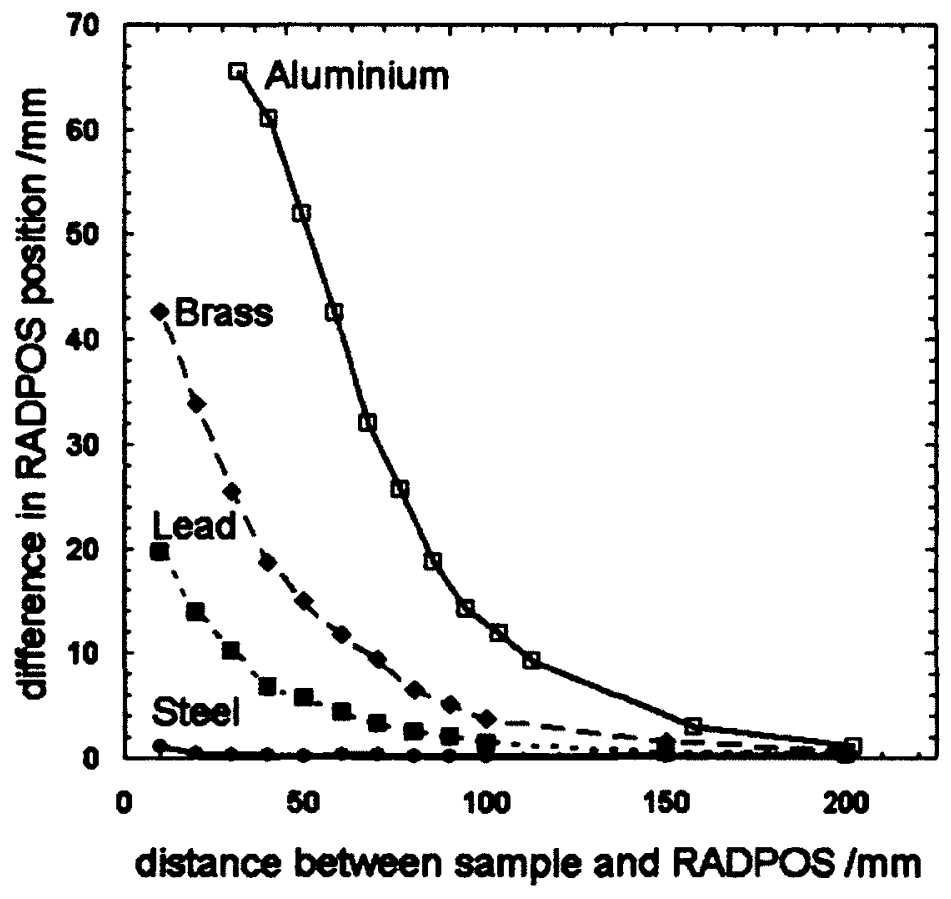

Figure 2.8. Results from metal interference test with $25 \times 25 \mathrm{~cm}^{2}$ samples that caused an effect on the RADPOS position (aluminium, brass, lead, steal). 
Results for all sample sizes followed the same trends as the magnitude of the perceived position shift decreased with decreasing sample size. For all samples, the distortions were reduced to an insignificant amount (within measurements uncertainty) when the separation between the detector and sample was $200 \mathrm{~mm}$. Non-magnetic stainless steel (Grade 304) caused a distortion of less than $2 \mathrm{~mm}$ when placed within 10 $\mathrm{mm}$ of the detector; therefore it can provide a reasonable alternative to other metals if required. When the samples were placed along line $a$, at various points away from the transmitter, the distortion followed the same fall-off pattern as seen in figure 2.8 . The magnitudes of the distortions were slightly less than what is seen in figure 2.8 , with a maximum positional error of $52 \mathrm{~mm}$ when the largest aluminium sample was within 10 $\mathrm{mm}$ of the transmitter. Since the only restriction on the placement of the transmitter is its distance from the detector, one should choose the transmitter position so that it is away from any potentially interfering materials and so that no such materials lie along the line collinear with the two pieces of equipment. Also, the distortion caused by a sample depends on the sample's physical orientation and shape, as the shift in position was much less when the sample was placed horizontally, with the large face of the square in-plane with the table top. The ferromagnetic property and electrical conductivity of a metal are the greatest indications of the magnitude of the distortion a sample will cause, as this can predict the magnitude of the interference with the magnetic field sent out by the transmitter. This interference will therefore alter the magnetic field detected by the sensor, distorting its perceived location.

Other studies have investigated the effect of metal interference on various versions of the 'Flock of Birds' electromagnetic tracking system. ${ }^{[27,40]}$. Cylindrical 
samples (12 mm diameter and $125 \mathrm{~mm}$ long) of aluminium, cobalt chrome alloy (Co-CrMo), mild steel (1018), stainless steel (SST $316 \mathrm{~L}$ ) and titanium alloy (Ti-6Al-4V), meant to mimic the shape and size of common upper-extremity orthopaedic implants have been used, as well as rods of stainless steel (SST 303 and SST 416), aluminium and brass that were $50 \mathrm{~mm}$ long and had a diameter of $12.7 \mathrm{~mm} \cdot{ }^{[27,40]}$ Both studies tested the materials at various positions along a line collinear with the detector and transmitter and compared the induced root-mean squared positional error (see equation 2.2) from each material. Both studies reported that a significant effect $(p<0.001)$ was found only from the specific steel samples (mild steel (1018) and stainless steel (SST 416)) that had the highest magnetic permeability. In both studies the distortion was greatest when the samples were placed near the sensor. For the shorter rods, the error reached a maximum of $5.26 \mathrm{~cm}$ when it was in contact with the sensor. ${ }^{[40]}$ For the longer steel rods, the error ranged from approximately $9 \mathrm{~mm}$, when the sample was placed adjacent to the transmitter, to more than $50 \mathrm{~mm}$, when it was placed adjacent to the detector. ${ }^{[27]}$ The steel samples with a lower magnetic permeability (SST 303 and 316) as well as titanium, cobalt chrome, aluminium and bronze had negligible effects. ${ }^{[27,40]}$.

The second study mentioned above also compared the interference seen with the microBird system to the effect of the different materials on another electromagnetic positioning system, Aurora ${ }^{\mathrm{TM}}$ (Northern Digital Inc., Waterloo, Ontario). ${ }^{[27]}$ The Aurora system showed smaller overall errors, however in contrast to the microBird system, distortion was at a maximum when the rods were close to the emitter, rather than the sensor. ${ }^{[27}$ Other studies have tested different medical tools such as metallic loops, wire guides, optical tracking tools, a drill, pliers, and a needle holder for interference with the 
Aurora system at various distances away from the transmitter. It was shown that these tools, including the metallic devices, can be used in close proximity to the detector without notable effects on the position accuracy. The drill did however cause root-mean squared positional error of approximately $2 \mathrm{~mm}$ when placed within $6 \mathrm{~cm}$ of the transmitter. ${ }^{[42]}$ 


\subsubsection{Preliminary clinical evaluation}

The RADPOS system was able to accurately measure the sinusoidal motion of the Quasar phantom's translation stage within $\pm 0.3 \mathrm{~mm}$ of the manually-set amplitude of 40 mm. Using the 'minimum time interval' setting, the system recorded a position measurement once every $30-40 \mathrm{~ms}$, which provided ample information to track the movement of the insert. Once it was noted that the RADPOS system could accurately measure consistent sinusoidal motion, various sample patient breathing files were used to drive the phantom in a pattern more representative of actual patient motion. Figure 2.9 shows comparisons between RADPOS measurements and two of the sample patient files that were used. The first plot shows data originally collected from a patient who displayed 'deep breathing' while the second plot was for 'irregular breathing'. It can be seen that when the phantom is at its lowest points in each cycle (seen on the plots as local minima), the RADPOS-read measurements slightly deviate from the patient file positions. This characteristic was investigated and appeared to be due to interference with the motor of the phantom, which contained metallic components. When the phantom is in the lower portion of the breathing cycles, it is closest to the motor and thus the interference is greater. A $5 \mathrm{~cm}$ block of acrylic was placed on top of chest wall platform to increase the distance between the RADPOS and the motor, however some residual effects remained. Even with this slight interference, the correlation between the RADPOS measurements and the patient data was 0.99 for the deep breathing pattern and 0.95 for the irregular breathing pattern. 
a)

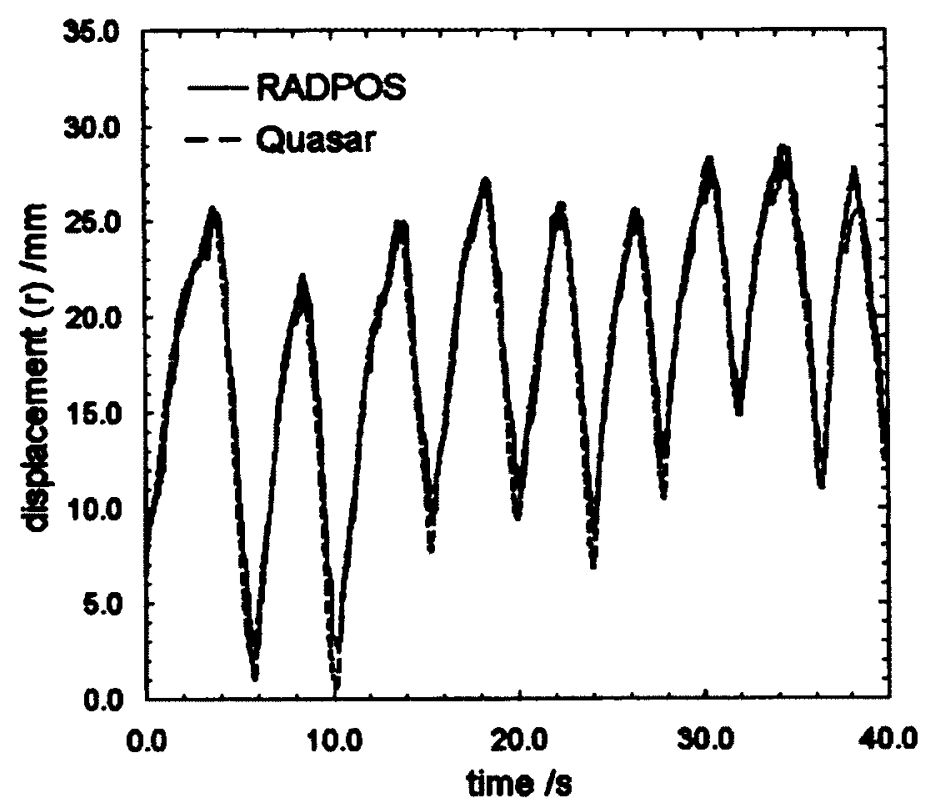

b)

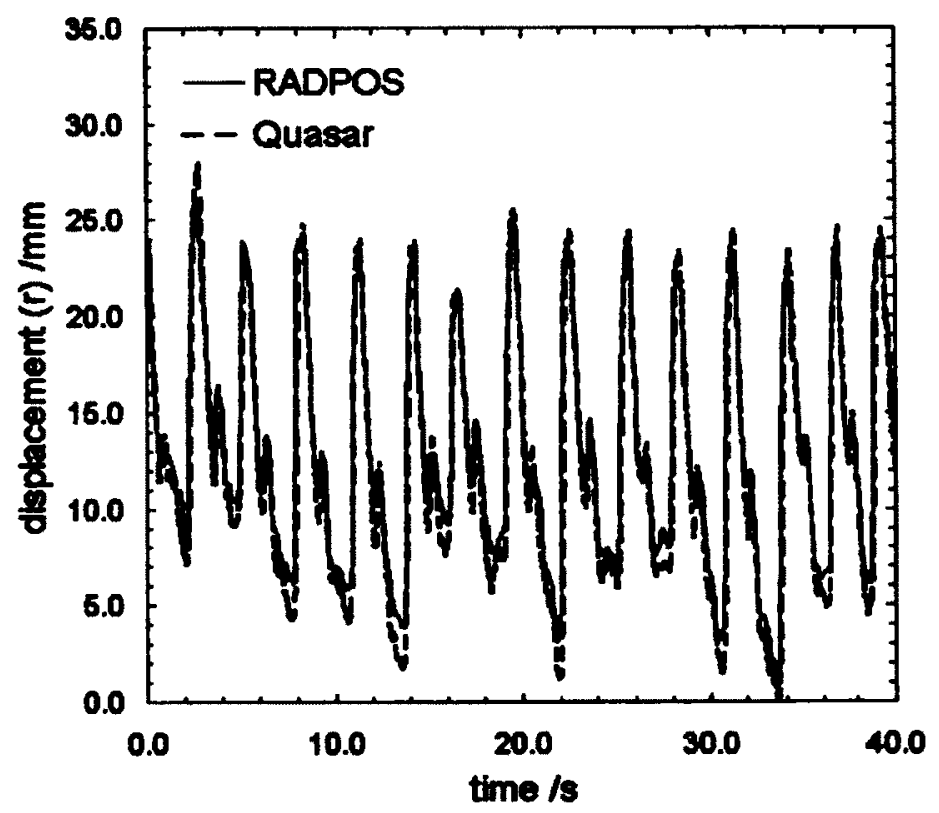

Figure 2.9. Respiratory motion produced by the Quasar phantom, as measured by the RADPOS detector. The phantom was moving according to sample patient files that displayed a) deep breathing and b) irregular breathing. 


\subsection{CONCLUSIONS}

Results of the preliminary tests indicate that the RADPOS system can be used for simultaneous position and dose measurements in ${ }^{60} \mathrm{Co}$ and high-energy beams from linear accelerators. Over the entire profile curve, the average absolute deviation between the RADPOS and diode relative dose profile measurements was $5.3,3.1$, and $2.9 \%$ of the maximum dose on the central axis for the ${ }^{60} \mathrm{Co}, 6 \mathrm{MV}$, and $18 \mathrm{MV}$ beams, respectively. The angular response of the probe over $360^{\circ}$ range is on average within $1.6 \%$, with a maximum deviation of $\pm 4 \%$, which is slightly higher than when microMOSFETs are used alone. ${ }^{[37]}$ The maximum deviation in the measured calibration coefficient for standard sensitivity MOSFETs was $-4.6 \%$ for large field sizes however the high sensitivity detector showed less of a change in response.

The position verification is stable within $0.21 \pm 0.07 \mathrm{~mm}$ over a period of an hour and can be used with the highest accuracy when interfering metals are at least $200 \mathrm{~mm}$ away. The optimal operating range for the detector is a volume defined by the following points (with respect to the transmitter): $150-470 \mathrm{~mm}$ along the $\mathrm{x}$-axis, $\pm 215 \mathrm{~mm}$ along the $y$-axis, and $\pm 100 \mathrm{~mm}$ along the z-axis. Within this volume, the uncertainty on $3 \mathrm{D}$ RADPOS displacements is $\pm 2 \mathrm{~mm}$. Preliminary clinical evaluation using a dynamic phantom to simulate patient motion due to breathing has shown the potential of the RADPOS system to accurately measure displacements similar to chest wall movement. 


\section{Chapter 3}

\section{D DOSE-POSITION VERIFICATION IN RADIATION THERAPY USING THE RADPOS SYSTEM IN A}

\section{DEFORMABLE LUNG PHANTOM}

\subsection{INTRODUCTION}

\subsubsection{Treatment of a moving target}

Tumour motion can cause artifacts in image-acquisition and limitations in treatment planning and delivery. ${ }^{[17]}$ During treatment delivery, tumour motion can cause a decrease in target dose, as the tumour moves with respect to the beam. This presents a particular challenge in 3D conformal radiotherapy and IMRT where the beam is specifically shaped to irradiate as little healthy tissue as possible. Organ motion can also cause an increase in dose to surrounding healthy tissues as they move into regions of high dose. Clinical evidence has shown the benefit to local control of the disease and to survival when higher dose levels are used. ${ }^{[48,49]}$ The use of higher dose levels must however be weighed against the increase in risk of complication to the healthy lung tissue, which has been shown to correlate with the mean lung dose. ${ }^{[50-55]}$

Several methods have been developed to address these issues, with the goal of maximizing target dose while minimizing further complications. The first and most widely used method is inclusion, where treatment margins are increased relative to the actual tumour size. ${ }^{[56]}$ This allows for the tumour to be covered with the prescribed dose 
throughout its predicted range of motion; however it can also lead to increased irradiation of healthy tissues, and therefore to a higher risk of toxicity. In addition, it assumes that the patient breathing pattern and tumour motion stay the same during the entire course of treatment, which frequently is not the case. ${ }^{[6,56-58]}$ Another method is to use gated beam delivery. ${ }^{[59]}$ In this case, radiation delivery is limited to when the target is within a certain range, where the tumour position is most stable and reproducible. The latest treatment methods developed involve real-time tumour tracking, where the beam is dynamically repositioned to account for tumour motion. The beam remains on the entire time, which increases the efficiency of treatment delivery, making for shorter treatment times than with gated radiotherapy. This type of treatment is called 4D radiotherapy and is defined as "the explicit inclusion of the temporal changes of anatomy during the imaging, planning and delivery of radiotherapy". ${ }^{[60]}$ Gating and 4D delivery methods are believed to provide the best opportunity of reducing tumour margins in cases of significant motion. ${ }^{[59]}$

\subsubsection{Verification of delivery methods}

With the recent increase of such delivery methods, the verification of different aspects of radiotherapy involving motion management is critical. ${ }^{[17]}$ Four-dimensional deformable phantoms have recently been introduced to simulate lung tissue deformation and tumour motion due to breathing during imaging and treatment delivery. ${ }^{[61-74]}$ The combination of such phantoms with in vivo dosimeters can be used to model patient breathing patterns and verify the accuracy of lung tumour treatments.

Other four dimensional phantoms have been created for verification of 4D radiotherapy, including both rigid and deformable models. ${ }^{[61.74]}$ Niotsikou et al., 
developed a lung phantom made out of bottles with accordion-type flexible and collapsible sides, mounted in a plastic thoracic cavity ${ }^{[69]}$ This phantom was used to compare treatment plan calculations and film measurements when the phantom was set to move with varying amplitudes. The goal of the study was to investigate the effects of fractionated radiation therapy on a moving anthropomorphic lung phantom and it was found that dose blurring occurred when the phantom was set to move with larger amplitudes. Vinogradskiy et al., combined a deformable phantom first described in Followill et al., with TLDs and film for verification of 4D dose calculations and found that results were within clinical standards. ${ }^{[66,70,71]}$ In these studies it is assumed that position information of the dosimeters can reliably be determined from pre-treatment CT simulation.

This chapter describes the combination of the RADPOS $4 \mathrm{D}$ in vivo dosimetry system with a deformable tissue equivalent lung phantom to simulate respiratory-induced tumour motion and to evaluate the potential use of the combined system as an effective quality assurance tool for $4 \mathrm{D}$ conformal radiotherapy. ${ }^{[47,75]}$ As the phantom simulated motion and deformation of lung tissue and a tumour during various stages of breathing, the RADPOS system was used to verify position and dose in real-time during treatment delivery. When ultimately applying this work to fully dynamic motion, the combined phantom-RADPOS dosimetry system could be extended to the quality assurance of conformal 4D treatment deliveries, providing feedback on the accuracy of the treatment delivery as well as any changes in dose to the target or simulated lung tissue due to changes in tumour position and breathing pattern. 


\subsection{MATERIALS AND METHODS}

\subsubsection{D deformable phantom}

The deformable phantom used for this project was developed by Serban et al., and consists of a balloon, which is surrounded by water and mounted in a Lucite cylinder. ${ }^{[75]}$ The balloon is filled with natural yellow sponges which are dampened to simulate the density of lung tissue. A small sphere made of a thermoplastic elastomer, Dermasol, is positioned inside the balloon and used as a tumour. The balloon is compressed and decompressed by a piston, which moves up and down along the superior/inferior direction. This emulates diaphragm motion in the body which causes 3D non-isotropic motion and deformation of the lung tissue. Figure 3.1 shows a schematic of the deformable phantom and also illustrates the contraction of the balloon due to the motion of the piston. This contraction/expansion of the balloon causes the tumour to move inside the phantom with a direction and magnitude that depends on the tumour's original position. 


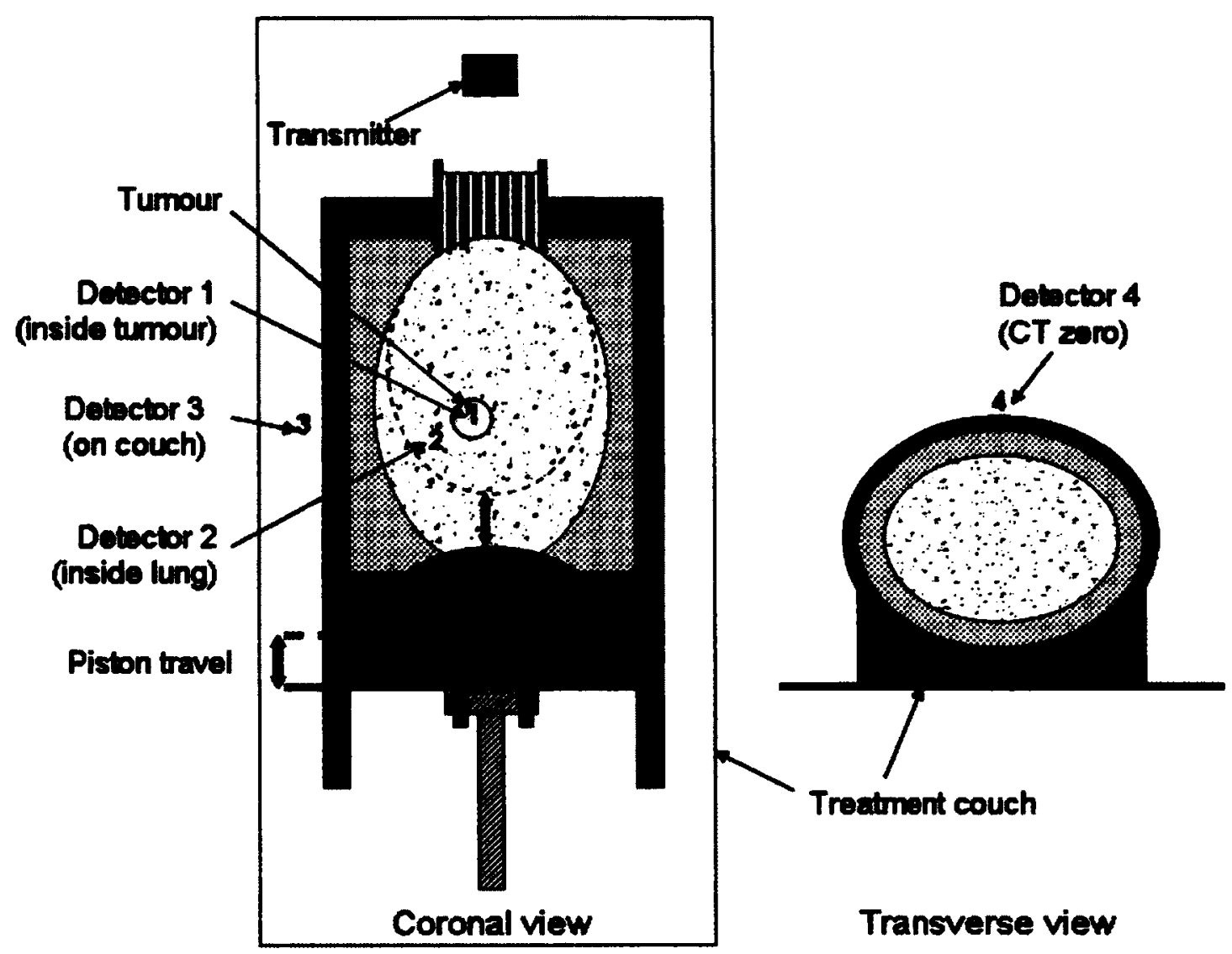

Figure 3.1. Schematic of the 4D deformable lung phantom and RADPOS detectors used in this work. Detectors 3 (on couch) and 4 (CT zero) remained stationary and were used as reference points throughout the experiment. The thick arrow showing piston travel is also used to indicate the deformation of the phantom balloon (shown as the dotted outline) for various breathing phases.

The range of motion of the piston has been broken up into 8 states based on a previous breakdown of the breathing cycle. ${ }^{[56]}$ As the piston moves the phantom from one extreme to the other (peak of inhale, POI, to peak of exhale, POE), the magnitude of the balloon deformations are $4 \mathrm{~mm}$ along the anterior/posterior axis, $5 \mathrm{~mm}$ along the left/right axis, and $20 \mathrm{~mm}$ along the superior/inferior axis. These deformations lead to changes in the mean density of the lung region of the phantom from $(0.19 \pm 0.12) \mathrm{g} / \mathrm{cm}^{3}$ for POI to $(0.24 \pm 0.12) \mathrm{g} / \mathrm{cm}^{3}$ for POE. ${ }^{[75]}$ The phantom motion in the superior/inferior direction is similar to the deformation of the lung in real patients due to movement of the 
diaphragm. Preliminary work has shown that the diaphragm can move 11 to $25 \mathrm{~mm}$ from POI to POE which can be simulated by the $20 \mathrm{~mm}$ superior/inferior motion of the phantom. ${ }^{[17,75]}$ Three particular piston positions (breathing states) were used in this study: 1) end of exhale, EOE, which is when the piston is at its highest point, and the balloon is therefore at maximum compression, 2) middle of inhale, MOI, when the piston is at the mid-point, and 3) end of inhale, EOI, when the piston is at its lowest point and the balloon is at maximum decompression.

\subsubsection{Position verification}

\subsubsection{CT scanner}

To study the reproducibility of the displacement of the tumour and lung tissue as the piston is moved to each position, the phantom was set-up on the couch of a Philips AcQSim CT scanner with two RADPOS detectors positioned inside the balloon, one directly inside the tumour and another one outside of the tumour, in the sponge simulating lung tissue, as shown in figure 3.1. To serve as reference position monitors, two more RADPOS detectors were placed outside the phantom, one on top of the Lucite cylinder and another on the treatment couch. The position of these reference detectors remains constant throughout different phantom breathing phases. Since the position of these detectors can be determined visually, their location in the CT or linac coordinate system is accurately known.

The piston was originally in the EOE position, so all RADPOS detector positions were zeroed at this position. The piston was then shifted to move the phantom into the other breathing states used for this work: MOI, and EOI. At each state, the position coordinates of all RADPOS detectors were recorded, and the displacement between states 
of the two detectors inside the phantom was calculated. The piston was then moved back to the EOE position and the procedure was repeated five times to verify that the position of the RADPOS detectors when the phantom was in a specific state did not drift or change significantly after the piston was moved to other positions.

The RADPOS system is an electromagnetically-based positioning system, potentially affected by proximity of metal objects, as investigated in Chapter $2 .{ }^{[47]}$ This effect was observed when the phantom approached the CT bore and the reported detector positions deviated from expected values. To quantify this effect, readings were taken with the phantom in each of the three states at various couch positions, from when the center of the phantom was almost two metres away from the CT bore to when it was in the middle of the bore, as shown as phantom positions 1 and 2 in figure 3.2. The positions of all RADPOS detectors, including the reference detectors on the outside of the phantom, were recorded at each horizontal couch position and compared. 


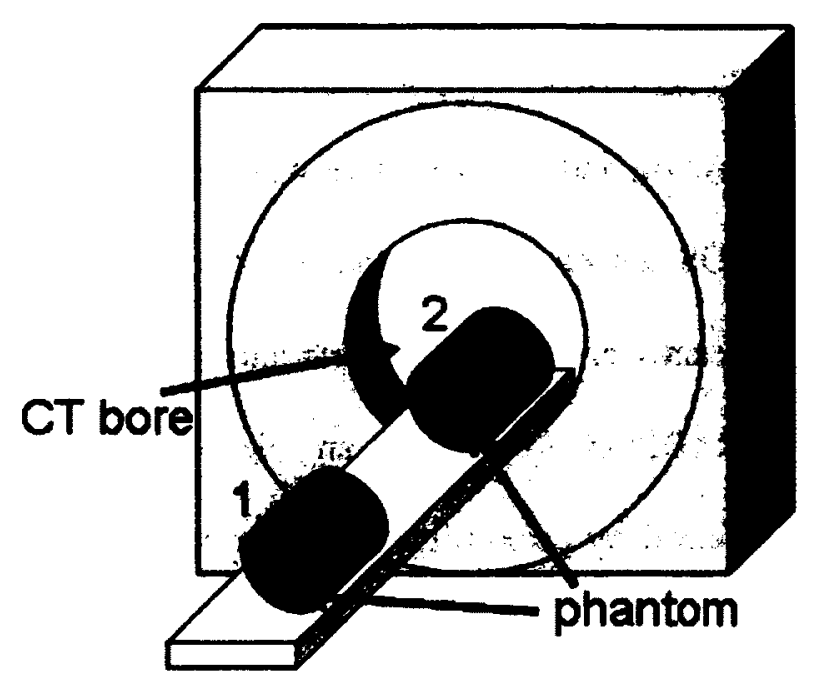

Figure 3.2. Deformable lung phantom on the couch of the CT scanner. To investigate interference from the CT on the RADPOS-read positions, the couch was moved to position the phantom as far as $2 \mathrm{~m}$ away from the CT bore to when the phantom was in the middle of the bore.

Three CT scans of $3 \mathrm{~mm}$ slices were then taken of the phantom in each of the three breathing phases: EOE, MOI, and EOI. The position of the RADPOS detector inside the tumour was determined by locating the positioning coil, which is visible on the scan, and approximating the location of the MOSFET detector, which is $8 \mathrm{~mm}$ from the end of the coil further down the probe wire. These positions were recorded and analyzed to determine the displacement of each RADPOS detector as the piston moved the phantom from one state to the next. This procedure was followed twice with the tumour at two different locations, the first time with the tumour at a more superior position, further away from the piston, and a second time with the tumour closer to the piston of the phantom. 


\subsubsection{Linac}

After the displacement of the detectors was measured with the phantom on the CT couch and scans were taken, the phantom was moved to the treatment room and placed on the couch of a Varian Clinac 6EX linac. The configuration of RADPOS detectors and transmitter was preserved with respect to the phantom. The piston was again moved to the EOE, MOI, and EOI positions, and the RADPOS positions were recorded. The position and displacement of each detector between the phases was measured and compared to those measured with the phantom on the CT couch.

To deliver different beam configurations, the linac gantry must rotate about an axis parallel to the treatment couch, as shown in figure 3.3. As the gantry rotates, the distance between the linac components and the RADPOS transmitter and detectors changes. To determine any effect the gantry angle had on the RADPOS-read positions, the gantry was rotated to six different positions ranging from $0-360^{\circ}$ with the phantom and RADPOS detectors on the linac couch. At each gantry position, the RADPOSmeasured position coordinates of the detectors were recorded and results were compared to determine any interference related to the position of the gantry. 


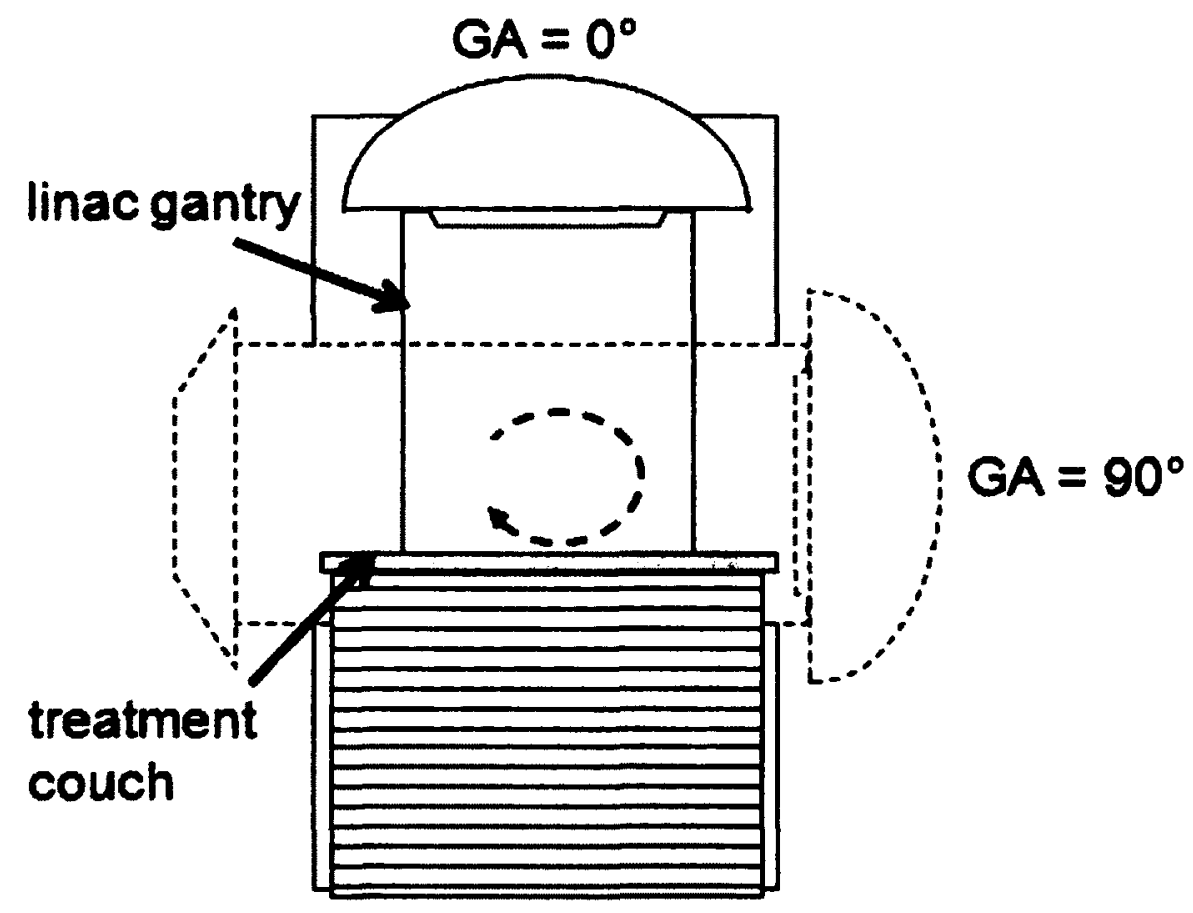

Figure 3.3. Gantry rotation about an axis parallel to the treatment couch.

\subsubsection{Dosimetric verification}

\subsubsection{Free-breathing experiment}

To evaluate the dosimetric characteristics of this system, the deformable phantom and RADPOS system was used in two radiation delivery scenarios. The first was a simulation of a free-breathing delivery. For this experiment, the treatment plan was created based only on the tumour and phantom geometry when the piston was at one position, which is just a static snapshot of the full phantom motion. The treatment was then delivered while the phantom "breathed normally", i.e. with the piston moved to generate the full phantom motion.

The treatment plan was created using one of the CT data sets taken with the phantom in the EOE phase, with the RADPOS detectors in the positions described above. 
The treatment plan was created using the XiO (CMS) planning system, and consisted of 3 beams angled: $0^{\circ}, 120^{\circ}$, and $240^{\circ}$. A clinical prescription of $2 \mathrm{~Gy}$ to the centre of the gross tumour volume, GTV, was assigned to be delivered in one fraction. A simplification of a patients' breathing pattern was modelled by dividing the phantom motion into two breathing states, EOE and EOI, and having the phantom spend an equal amount of time in both states. The treatment was delivered in two parts, with the phantom in EOE for the first half, and then moved to EOI for the second half. The two parts of the treatment were delivered consecutively with the two detectors remaining in the same position, one inside the tumour and another inside the sponge (lung) region of the phantom for the entire treatment delivery. The two reference detectors also remained in position. After each beam was delivered, RADPOS dose measurements were made and these values were compared to the treatment plan dose values at the point of each detector.

\subsubsection{Adaptive delivery experiment}

The second delivery scenario was a simulation of an adaptive treatment, which involves modifying the treatment according to the tumour motion during delivery. In this case, specific treatments plans were created and delivered based on various stages of the phantom motion. This involved creating three different treatment plans using the EOE, MOI, and EOI CT datasets. To simulate a delivery where the treatment beam could follow the tumour, the phantom was set-up on the treatment couch and after the piston was moved to each breathing phase position, the treatment plan specific for that phase was delivered. The RADPOS-reported positions of the detector inside the tumour, relative to the reference detector, were noted for each phase position of the phantom. A ball bearing (bb) was also placed at the location of the reference detector, CT zero, so this 
point was easily located on the CT scan. The relative shifts from this point were then used to locate the position of the detector on the treatment planning system. It was at this point that the treatment planning system calculated dose was compared to dose measurements.

\subsection{RESULTS \& DISCUSSION}

\subsubsection{Position Verification}

\subsubsection{CT scanner}

The reproducibility of the positions of the RADPOS detectors inside the phantom is shown in figure 3.4. For all trials, the position of each detector in a given phase remained within $0.9 \mathrm{~mm}$ of the other positions recorded for that detector and phase. There was no significant drift in the position of Detector 1 , which was inside the tumour, over time as the piston was moved then returned to a given phase. The position of Detector 2 , which was placed inside the sponge region of the phantom, shifted slightly in each phase over time. Detector 1 was securely mounted inside the tumour, while Detector 2 was positioned among the sponge and not attached to any rigid material. 
a)

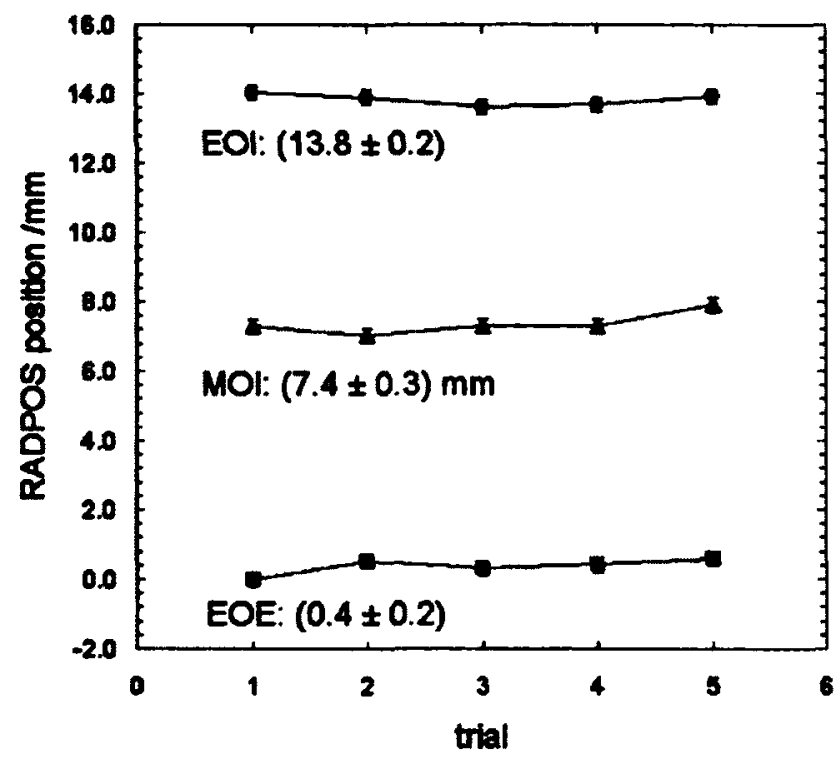

b)

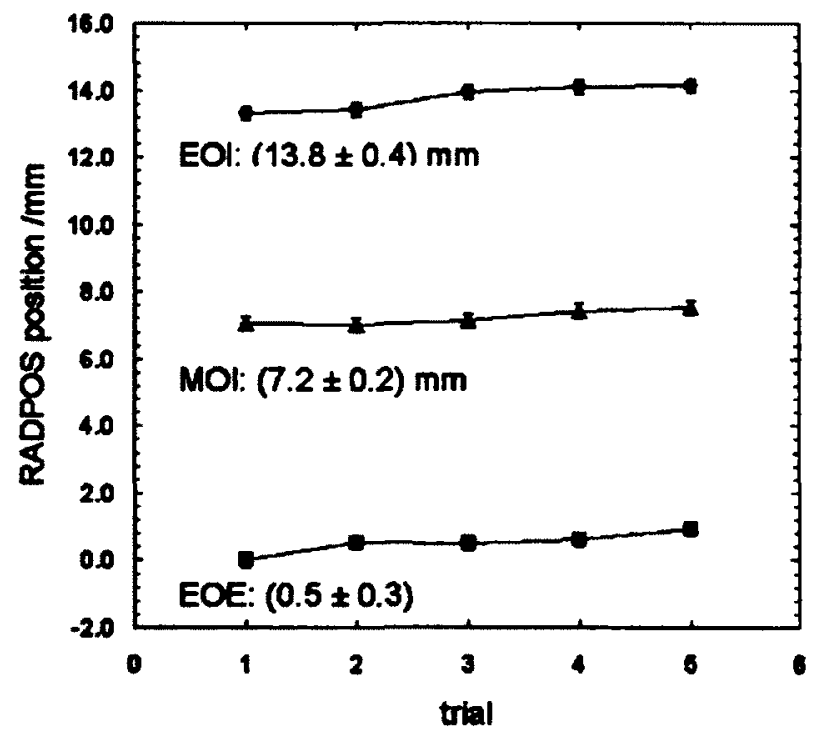

Figure 3.4. Reproducibility of RADPOS positions with the piston in EOE, MOI, and EOI states for a) Detector 1 , inside the tumour and b) Detector 2, inside the lung tissue. For each detector, the positions were zeroed at the original EOE position. The average and standard deviation of the position at each stage is shown on the plot. For all trials, the position of each detector in a given phase remained within $0.9 \mathrm{~mm}$ of the other positions recorded for that detector and phase. 
It should be noted that the inherent reproducibility of the deformable phantom when moving through different phases, was shown to be within image resolution of $0.7 \times 0.7 \times 1.25 \mathrm{~mm}^{3}$ and positional reproducibility of the RADPOS system is better than $0.21 \pm 0.07 \mathrm{~mm} .{ }^{[75]}$ We therefore conclude that the shift observed on Detector 2 is within the reproducibility of the system, however, attaching the detectors inside the phantom more securely to the sponge may eliminate potential problems and allow the positions to be more reproducible.

The relative displacement of each detector between the EOI-MOI, MOI-EOE, and EOE-EOI phases was calculated from the RADPOS-measured coordinates at each couch position. Over the full range of horizontal motion of the couch, the RADPOS-measured displacements remained constant within an average of $(0.4 \pm 0.1) \mathrm{mm}$ for both detectors with no deviation greater than $0.8 \mathrm{~mm}$ for the detector inside the tumour and $0.9 \mathrm{~mm}$ for the detector inside the lung tissue of the phantom.

The displacement of the detector inside the tumour between phases, as measured by the RADPOS system, was compared to the information on the CT images. The position of the detector was determined by examining each of the three scans taken of the phantom and recording the coordinates where the positioning sensor appeared to be located. The displacement between the three phases was then calculated and compared to the RADPOS-measured displacements, as seen in table 3.1. The agreement between the RADPOS measurements and the CT images was within $1.5 \mathrm{~mm}$ and $2.5 \mathrm{~mm}$ for the first and second detector locations, respectively. Considering the uncertainty of the RADPOS system $\left(\sigma_{\text {RADPOS_3D }}=2.0 \mathrm{~mm}\right)$ and the uncertainty due to the slice thickness of the CT scans $\left(\sigma_{\mathrm{CT}}=1.5 \mathrm{~mm}\right)$, the larger value seen for the second trial may have been due to 
interference from the piston driving rod, since the detector was closer to it than during the first trial.

Table 3.1. RADPOS displacement vector magnitudes in the three piston positions (EOE, MOI, EOI) measured by the RADPOS system on the CT couches and as determined from the CT image set. Measurements were taken twice, the first time with the tumour at a more superior position, further away from the piston (location 1), and a second time with the tumour closer to the piston of the phantom (location 2).

\begin{tabular}{|c|c|c|c|c|}
\hline \multirow{2}{*}{$y_{n+1}$} & \multicolumn{2}{|c|}{ Detector 1: Inthe tumour } & \multicolumn{2}{|c|}{ 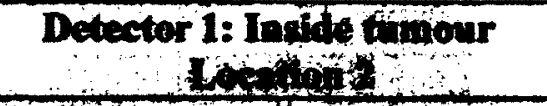 } \\
\hline & $H_{n}=(-1)$ & $r\left(n_{m p m}(m)\right.$ & ranes (mol) & 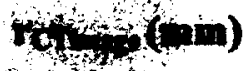 \\
\hline EOE-MOI & 4.3 & 5.8 & 12.0 & 9.5 \\
\hline MOI-EOI & 3.0 & 2.9 & 9.9 & 11.9 \\
\hline EOI-EOE & 7.4 & 8.7 & 21.8 & 21.4 \\
\hline
\end{tabular}

\subsubsection{Linac}

For the RADPOS system and deformable phantom to act as a potential quality assurance device in $4 \mathrm{D}$ radiation therapy, the coordinates reported by the system must be preserved between simulation and delivery. To verify this, the displacement vectors of the two RADPOS detectors inside of the phantom were calculated as the piston was moved between the three phases on both the CT and linac couches, and the results are shown in table 3.2. The displacements measured by the RADPOS system in both locations were on average within $(0.7 \pm 0.3) \mathrm{mm}$, with a maximum deviation of $1.0 \mathrm{~mm}$. Since the uncertainty of RADPOS-measured displacements within this range is \pm 2.0 $\mathrm{mm}$, the system can provide relative displacement measurements in the treatment room, in proximity to the linac, that are consistent with measurements made in the CT scanner room. Therefore, the relative coordinates taken on the linac and CT couches can be accurately linked. It is also important to note that the absolute position readings of the 
detectors taken on the linac couch were consistent with the absolute readings taken on the CT couch, with the couch far from the bore, where the electromagnetic interference is minimal.

Table 3.2. The displacement vector magnitudes of the two detectors inside the phantom measured by the RADPOS system on the CT and linac couches. The displacement vector for each trial is shown (ex. $r_{C T}=\sqrt{x_{C T}{ }^{2}+y_{C T}{ }^{2}+z_{C T}^{2}}$ ) as well as the difference between the measurements made on the two different couches $\left(\Delta r=\sqrt{\left(x_{C T}-x_{\text {beac }}\right)^{2}+\left(y_{C T}-y_{\text {max }}\right)^{2}+\left(z_{C T}-z_{\text {binax }}\right)^{2}}\right)$.

\begin{tabular}{|c|c|c|c|c|c|c|}
\hline+40 & $\frac{p}{\operatorname{rcr}\left({ }^{2}+1\right)}$ & $\lim (\operatorname{in})$ & $\frac{1}{\Delta r(a, a)}$ & $\begin{array}{l}\text { rar (nim) } \\
\operatorname{ran}\end{array}$ & naviof (mai) & $x^{3}$ \\
\hline EOE-MOI & 6.5 & 6.8 & 0.4 & 4.7 & 4.8 & 0.2 \\
\hline MOI-EOI & 7.5 & 6.9 & 0.7 & 5.2 & 4.5 & 0.9 \\
\hline EOI-EOE & 14.1 & 13.3 & 0.9 & 9.9 & 8.9 & 1.0 \\
\hline
\end{tabular}

The effect of gantry angle on the RADPOS-measured positions both relative to the transmitter (in absolute terms) and relative to a reference detector (relative terms) were investigated. Figure 3.5 shows the effect of the linac gantry angle on the RADPOSmeasured relative positions. The gantry angle affected the readings of the four detectors by varying magnitudes; however the induced shifts followed approximately the same pattern as the gantry was rotated. The two reference detectors on the outside of the phantom, closest to the gantry, were affected the most, with absolute shifts up to $(1.3 \pm$ $0.1) \mathrm{mm}$ and $(2.2 \pm 0.2) \mathrm{mm}$ at a gantry angle of $250^{\circ}$. Despite this, as indicated in figure 3.5 , the variation of detector position when measured relative to a reference detector is strongly reduced as all detectors qualitatively vary in the same manner, albeit not with the exactly same magnitude. The difference of the relative position of the detectors in the 
phantom averaged over the EOI, MOI and EOE states is no more than $1.1 \mathrm{~mm}$ compared to the relative position at gantry angle $120^{\circ}$. The reason for the shift in reported absolute position was again interference to the electromagnetic transmitter caused by the proximity to the gantry.

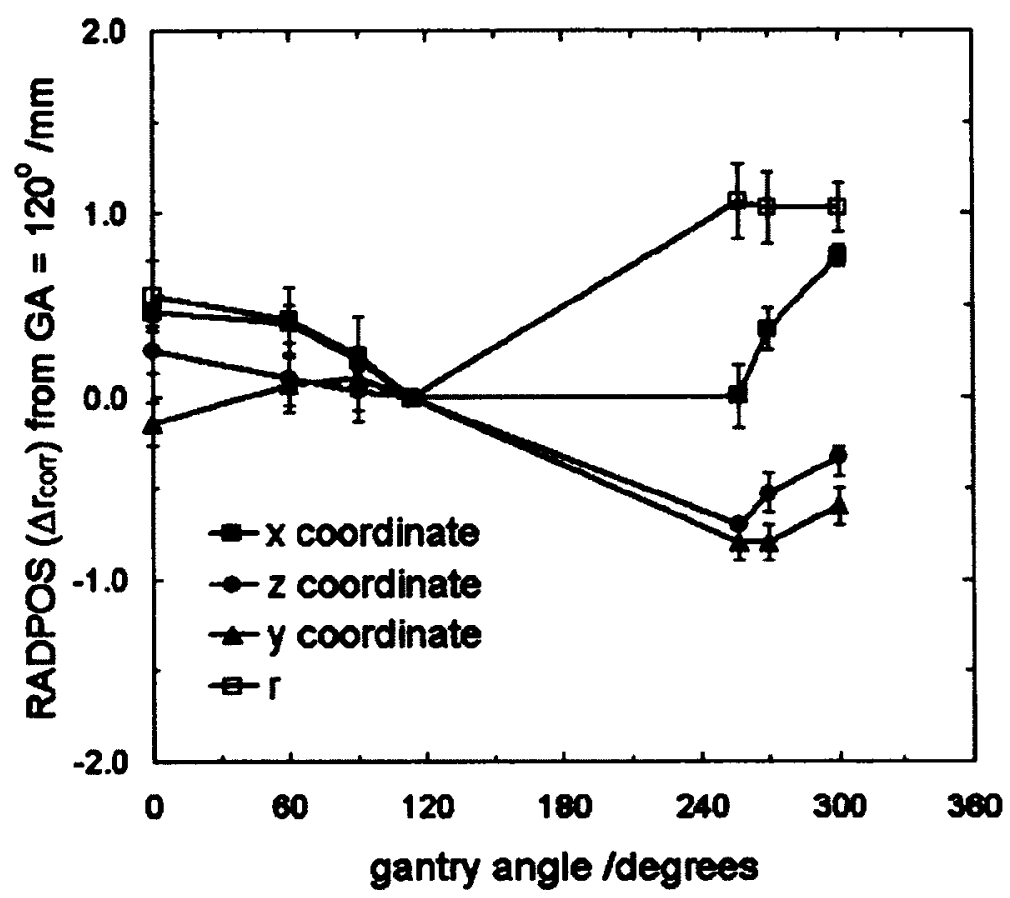

Figure 3.5. Difference of relative RADPOS position readings at various angles from reading at gantry angle (GA) $=120^{\circ}$, (eg. $\left.x_{\text {Den, }, G A}^{\prime}=\left(\left(x_{D e n, G A}-x_{\text {ref,GA }}\right)-x_{\text {Det1,120 }}\right)\right)$ for the detector inside the tumour. Data shown are averaged over all three phases (EOE, MOI, EOI), and error bars represent the standard deviation of the averaged values.

\subsubsection{Dosimetric Verification}

\subsubsection{Free-breathing experiment}

The goal of this part of the study was to show that the dynamic lung phantom can be used to simulate intra-fraction tumour motion of a lung cancer patient, simplifying the breathing motion in this case into two distinct states, EOE and EOI, and having the phantom spend equal time in both states. The RADPOS system can then be used as a 
quality assurance tool for a given treatment plan, providing dose and position information for the tumour as well as for other regions of interest. Figure 3.6 shows the beam eye view of the tumour volume (GTV) with the anterior $0^{\circ}$ beam for the treatment plan with the phantom in a) EOE phase and b) EOI phase for the free-breathing experiment. Figure 3.6 also shows the corresponding dose volume histograms, DVH's (panel $\mathrm{c}$ and d), for the two halves of the treatment delivery with the dashed curve representing the dose to the lung and the solid curve representing the dose to the tumour. The DVH for the EOE phase, the phase for which the plan was created, shows that $100 \%$ of the tumour volume is getting $95 \mathrm{cGy}$. From figure 3.6a, it can be seen that this is when the tumour is perfectly centred in the beam. Figure $3.6 \mathrm{~b}$ shows the beam eye view for the EOI phase, where the tumour is partially out of the beam. This caused a decrease in the dose to the tumour since the beam was not modified to account for this motion. The corresponding DVH curves in figure 3.6c and d show this degradation of dose to tumour with $100 \%$ of volume only getting approximately $10 \mathrm{cGy}$ in figure $3.6 \mathrm{~d}$, rather than $95 \mathrm{cGy}$ as planned. The dose to the lung was virtually the same in the EOI phase as for EOE, so there is less dose to the target due to a partial geographic miss while the surrounding healthy tissue receives no additional sparing. 
a)

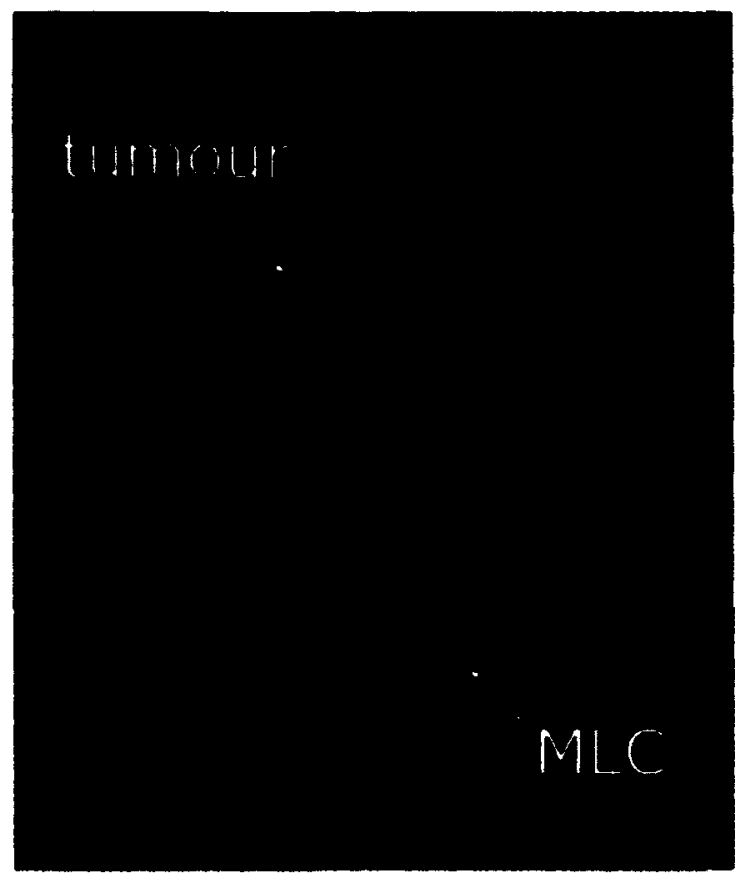

c)

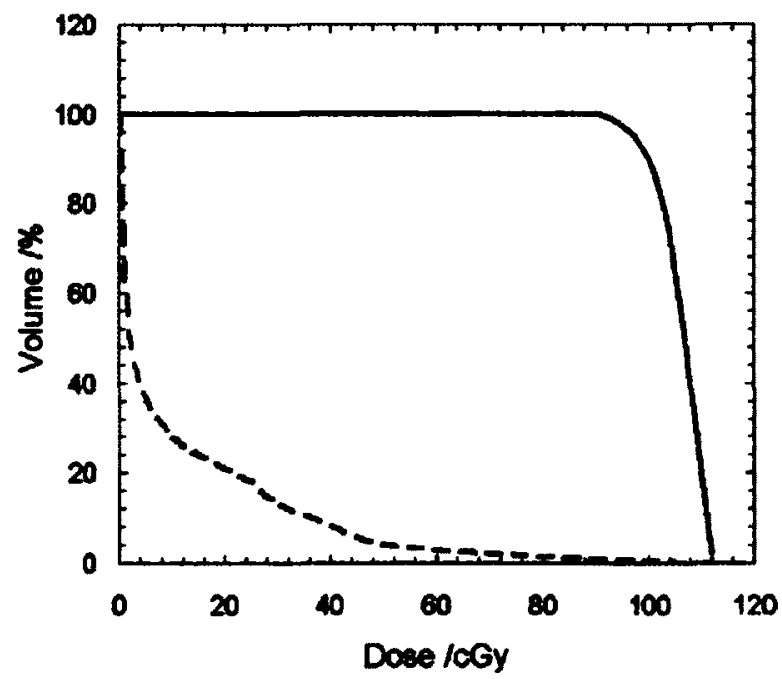

b)

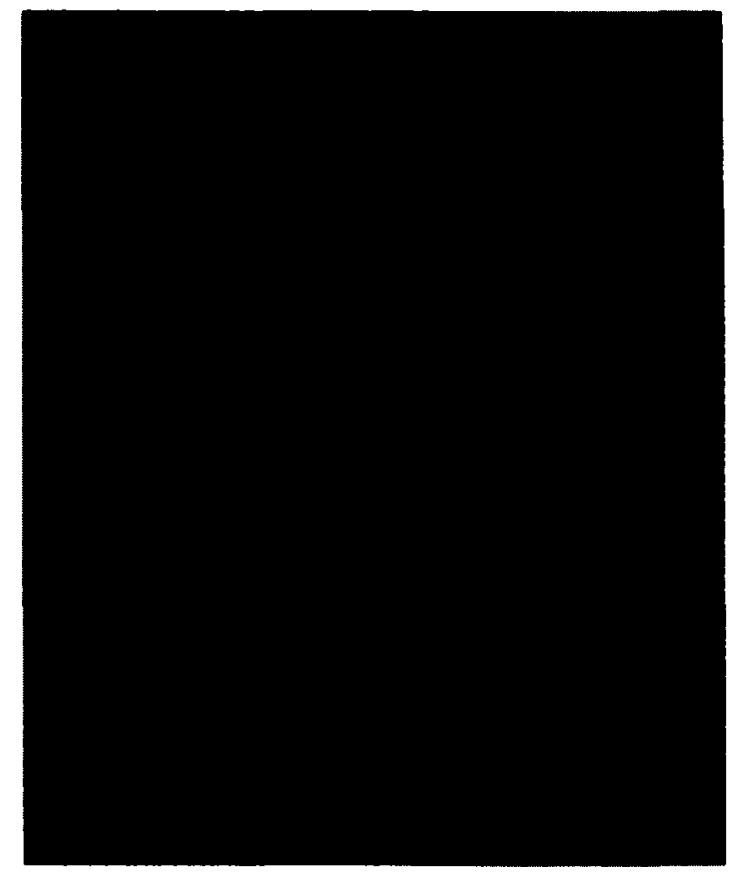

d)

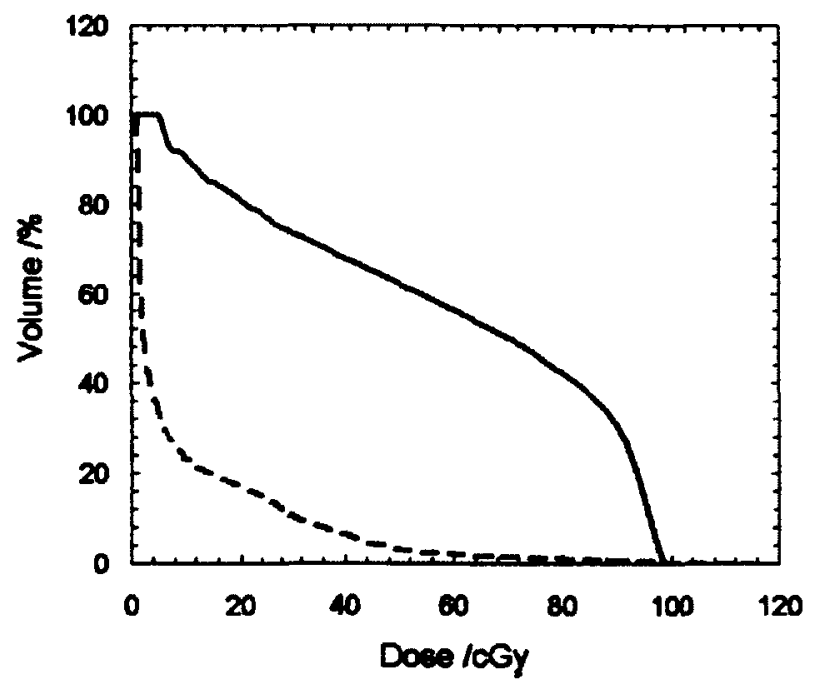

Figure 3.6. The beam eye view for treatment delivery with the anterior $0^{\circ}$ beam for a) 1st state: phantom in EOE phase (position used for planning) and b) 2nd state: phantom in EOI phase (tumour now partially out of primary beam). Dose volume histograms are also shown for the two halves of the treatment: c) EOE and d) EOI. The dashed curve shows the dose to the lung and the solid curve represents the dose to the tumour. 
The dose measurements from the three individual beams taken by Detector 1 (inside the tumour) and Detector 2 (inside the lung region) are shown in table 3.3 along with the treatment plan values. The location of the detector on the CT scan used to determine treatment plan values was approximated by locating the positioning coil, which is visible on the scan, and approximating the location of the sensor, which is $8 \mathrm{~mm}$ from the end of the coil along the direction of the probe. The absolute difference in measurements for individual beams ranges from -2.2 to $4.2 \mathrm{cGy}$. In some cases this results in a large percentage difference due to the low absolute value, for example the $0^{\circ}$ and $240^{\circ}$ beams for Detector 1 measurements in the EOI phase. The total dose reported by RADPOS agrees within $4 \%$ and $5 \%$ of the treatment planning doses in the tumour and the lung portion of the phantom, respectively. These differences are reasonable given different sources of uncertainty such as RADPOS positioning and dose uncertainties, the uncertainties associated with the treatment plan dose values, and the linac output reproducibility. It was estimated that these combined uncertainties amount to about $5 \%$. 
Table 3.3. RADPOS-measured dose values and treatment plan doses for the EOE and EOI phases of the free-breathing experiment. Data are shown for a) Detector 1 : inside the tumour and b) Detector 2 , inside the lung portion of the phantom.

a)

\begin{tabular}{|c|c|c|c|c|c|}
\hline \multirow{2}{*}{ Fhanes } & \multirow{2}{*}{ Demin } & \multicolumn{2}{|c|}{ Dase (cGy) } & \multicolumn{2}{|c|}{ Difforence (apros - TP) } \\
\hline & & RADPOS & Traatuneat Plan & cGy & $\%$ \\
\hline EOE & $0^{\circ}$ & 41.7 & 37.5 & 4.2 & 10.6 \\
\hline EOE & $120^{\circ}$ & 37.4 & 36.0 & 1.4 & 3.9 \\
\hline EOE & $240^{\circ}$ & 35.3 & 36.5 & -1.2 & -3.3 \\
\hline \multicolumn{2}{|c|}{ Total } & 114.4 & 110.0 & 4.4 & 4.0 \\
\hline EOI & $0^{\circ}$ & 9.4 & 12.7 & -3.3 & -30.2 \\
\hline EOI & $120^{\circ}$ & 12.4 & 12.5 & -0.1 & -0.4 \\
\hline EOI & $240^{\circ}$ & 14.7 & 12.3 & 2.5 & 18.1 \\
\hline \multicolumn{2}{|c|}{ Total } & 36.5 & 37.5 & -1.0 & -2.6 \\
\hline
\end{tabular}

b)

\begin{tabular}{|c|c|c|c|c|c|}
\hline \multirow{2}{*}{ ind } & \multirow{2}{*}{ Divary } & \multicolumn{2}{|c|}{$\operatorname{Doms}(\alpha g)$} & \multicolumn{2}{|c|}{ Difference onahos - ms } \\
\hline & & RADWB & Thutriest Ptom & cy & $\%$ \\
\hline EOE & $0^{\circ}$ & 14.4 & 14.5 & -0.1 & 0.9 \\
\hline EOE & $120^{\circ}$ & 19.9 & 21.8 & -1.9 & 9.4 \\
\hline EOE & $240^{\circ}$ & 32.0 & 32.4 & -0.4 & 1.4 \\
\hline \multicolumn{2}{|c|}{ Total } & 66.3 & 68.7 & -2.4 & -3.6 \\
\hline EOI & $0^{\circ}$ & 5.5 & 5.0 & 0.5 & 9.4 \\
\hline EOI & $120^{\circ}$ & 4.9 & 7.1 & -2.2 & -37.7 \\
\hline EOI & $240^{\circ}$ & 29.1 & 29.5 & -0.4 & -1.3 \\
\hline \multicolumn{2}{|c|}{ Total } & 39.5 & 41.6 & -2.1 & -5.3 \\
\hline
\end{tabular}




\subsubsection{Adaptive delivery experiment}

For the adaptive delivery, the tumour was always within the beam, similar to figure 3.6a. This is because the treatment plan was tailored to suit each breathing phase, so the beam moved to compensate for the tumour motion. Table 3.4 shows the RADPOS dose measurements taken by the detector inside the tumour during the adaptive delivery experiment. Measurements were taken after all three beams of each treatment plan were delivered. The relative coordinates of the RADPOS detector inside the tumour were used to determine the point at which treatment plan dose values were compared, as described in the Material and Methods section of this chapter. The RADPOS-measured dose values were within $1.5 \%$ of the treatment plan values, which is well within the estimated experimental uncertainties. This agreement is better than the results from the freebreathing experiment, where the treatment plan was created for the EOE phase, and then delivered to both the EOE and EOI phase. The largest discrepancies between dose measurements and treatment plan calculations were found for the free breathing treatment of the EOI phase, which could potentially be due to the fact that the tumour had moved partially out of the beam in this position, through a high dose gradient area. This resulted in a much lower dose at the measurement/calculation point. The high dose gradient along with increased uncertainty of MOSFET measurements at low doses could have caused the measurements and dose calculations to be more vulnerable to slight inaccuracies in positioning and to limitations in the treatment planning calculations. For the adaptive experiment, a separate treatment plan was created for each phase so the tumour was centered in the beam for each delivery. This resulted in higher measured/calculated doses which lead to smaller statistical uncertainties. 
Table 3.4. RADPOS-measured dose values for the EOE, MOI, and EOI phases of the adaptive treatment experiment. Data are shown for Detector 1 , inside of the tumour.

\begin{tabular}{|c|cc|cc|}
\hline \multirow{2}{*}{ Phase } & \multicolumn{2}{|c|}{ Dose (eGy) } & \multicolumn{2}{c|}{ Difference (auderos - m) } \\
\cline { 2 - 5 } & RABPOS & Treatineint Plan & eGy & $\%$ \\
\hline EOE & 62.7 & 63.3 & -0.6 & -1.0 \\
MOI & 64.2 & 64.5 & -0.3 & -0.5 \\
EOI & 67.8 & 66.8 & 1.0 & 1.5 \\
\hline Total & 194.7 & 194.6 & 0.1 & 0.1 \\
\hline
\end{tabular}

\subsection{CONCLUSIONS}

The viability of a system consisting of RADPOS detectors and a deformable tissue-equivalent lung phantom to act as a quality assurance tool for four-dimensional radiation therapy has been investigated. The positional accuracy of the RADPOS detectors was estimated to be within $2.5 \mathrm{~mm}$ and relative displacement measurements were preserved between CT simulation and radiation delivery to within an average of $(0.7$ $\pm 0.3) \mathrm{mm}$. This shows that the relative coordinates of the detectors inside the phantom as reported by the RADPOS system are in agreement with detector coordinates derived from CT images. These relative coordinates were also preserved upon dose delivery from a free breathing and adaptive treatment plan. The system was subjected to radiation delivery in free-breathing and adaptive radiotherapy scenarios. For the free-breathing delivery, the total dose reported by RADPOS agreed to within $4 \%$ and $5 \%$ of the treatment planning doses in the tumour and the lung portion of the phantom, respectively. The RADPOS-measured dose values for the adaptive delivery were within $1.5 \%$ of the treatment plan values, which was well within the estimated experimental uncertainties. This work has shown that the deformable lung phantom-RADPOS system can be an 
efficient quality assurance tool for $4 \mathrm{D}$ radiation therapy serving as an end-to-end verification instrument. The combined system can be used to simulate patient motion and verify the accuracy and reproducibility of treatment techniques. 


\section{Chapter 4}

\section{REAL-TIME MEASUREMENTS OF PATIENT MOTION AND}

\section{DOSE DURING EXTERNAL BEAM RADIATION THERAPY}

\section{TREATMENTS FOR LUNG CANCER}

\subsection{INTRODUCTION}

\subsubsection{Lung cancer and respiratory motion}

Malignant lung disease is the leading cause of death due to cancer, responsible for over one-quarter of all cancer deaths. ${ }^{[1]}$ It is the second most common cancer among both men and women and is out-ranked in prevalence only by prostate cancer for men and breast cancer for women. ${ }^{[1]}$ It is projected that there will be 25,300 new cases and 20,600 deaths from lung cancer alone among Canadians in the year 2011. ${ }^{[1]}$ Radiation therapy is used for both curative and palliative treatment of lung cancer, with cure possible in stages I-III non-small-cell lung cancer, NSCLC, and in limited-stage small-cell lung cancer, SCLC. ${ }^{[76]}$ Local failure and distant metastases remain a concern however for lung cancer patients of all stages. ${ }^{[76]}$

Studies have shown benefits to local control with elevated dose, but high dose levels must be limited to the target only and not compromise nearby healthy tissues. ${ }^{[3,7]}$ Respiratory-induced tumour motion is a main concern in radiation treatment planning of lung cancer patients which limits the prescription of higher dose levels. ${ }^{\left[{ }^{3]}\right.}$ Several studies have aimed to quantify lung tumour motion and a general consensus is that the principal 
component of motion is in the superior-inferior, SI, direction, with a study by Chen et al. reporting motion amplitude of lung tumours as high as $50 \mathrm{~mm} \cdot{ }^{[78]} A$ report on the management of respiratory motion in radiation oncology by Task Group 76 recommends that tumour motion be measured whenever respiratory motion is perceived to be a concern. If the tumour motion is greater than $5 \mathrm{~mm}$, respiratory motion compensation techniques should be employed to increase normal tissue sparing and to avoid partial geographic miss of the tumour. ${ }^{[17]}$ One method to compensate for respiratory motion is to plan according to a maximum-intensity projection of the target from a 4DCT scan. Respiratory correlated $4 \mathrm{DCT}$ is used during treatment planning to define treatment volumes based on the displacement of the tumour during the scan. While this process assumes that the patient's breathing pattern (and therefore tumour motion) measured on the day of the planning CT remains relevant throughout the entire treatment process, it has been shown that this assumption is not always valid. ${ }^{[7,79,80]}$ Tumour motion can be unpredictable and vary over the course of treatment as well as during a single fraction ${ }^{[77]}$ resulting in increased irradiation of healthy tissue, a geographic miss of the target or both. ${ }^{[81]}$ Image-guidance systems such as cone-beam computed tomography, CBCT, can be used to verify patient position before treatment begins. This reduces the risk of set-up errors, which can have large consequences on delivered dose. ${ }^{[82]}$ It does not, however, account for intra-fraction motion throughout the duration of the treatment, which can be between 15-45 minutes for some image-guided IMRT treatments. ${ }^{[18]}$ Treatments on the Cyberknife unit can be even longer; lasting more than 3 hours. ${ }^{[83]}$ 


\subsubsection{Motion monitoring}

Tumour motion during treatment can be directly measured by implanting fiducial markers into the tumour. The movement of these fiducials can then be tracked using fluoroscopy, which delivers extra dose to the patient. The implantation of fiducials is also associated with higher levels of patient morbidity. ${ }^{[84]}$ It is more practical in the clinic to measure a surrogate for tumour motion. Commonly used methods of external motion measurement during treatment include spirometry, ${ }^{[85]}$ infrared markers, ${ }^{[86]}$ and electromagnetic positioning systems. ${ }^{[7]}$ A spirometer is a device that measures air flow in and out of a patient's mouth. This provides an indirect measure of lung volume but no quantitative measurements of position changes. Infrared markers can be placed on a patient's chest and are used in conjunction with cameras inside the treatment room to measure external surface motion. Qualitative information can be extracted; however the markers must be continuously visible to numerous cameras to enable 3D data collection.

Electromagnetic positioning systems measure a detector's response to a transmitted magnetic field to calculate 3D position displacements. They provide similar data to optical tracking systems, however they benefit from several advantages such as low cost, ease of calibration, and quick set-up. ${ }^{[7]}$ The major disadvantage of such systems is the interference caused by metals, as described in detail in Chapter 2. Special corrections have to be applied when metallic materials are in close vicinity to the transmitter or position sensor. ${ }^{[37,47]}$ Optical marker-based systems do not suffer this disadvantage.

Numerous studies have been dedicated to the correlation between external markers and internal target motion ${ }^{[56,86-93]}$ and while the relationship is not consistently simple and linear, it is believed that sufficient evidence exists to support external 
measurements. The wide range of findings on this topic suggest that one cannot necessarily assume external excursion equals internal excursion; however external motion measurements can provide a means of detecting changes in overall breathing patterns and possible deviations from data collected at the time of a planning CT ${ }^{[56,86-93]}$ External motion measurements can also be used to coach the patient to breathe in a more consistent manner, which has been shown to improve treatment reproducibility. ${ }^{[94]}$

Until this point, the investigation of performance characteristics of the RADPOS system was conducted using only phantoms. ${ }^{[88,89]}$ The goal of the study presented in this chapter was to use the RADPOS 4D in vivo dosimetry system to characterize the breathing patterns of lung cancer patients throughout the course of treatment and to measure inter-fraction variations in skin dose. Within this framework, the feasibility of general use of the RADPOS system on patients during daily treatment fractions was also assessed.

\subsection{MATERIALS AND METHODS}

\subsubsection{Phantom study}

Interference with the CT bore can cause shifts and increase noise in the position data collected on patients during the 4DCT scans. Correction methods have been investigated to reduce these effects; however results were not ideal for comparison between the RADPOS and Philips Bellows systems in all patient cases due to time needed to collect the data, and limitations in system-set-up. Since the Philips Bellows system only records data during the scan, it was decided to use a $4 \mathrm{D}$ phantom to simulate motion due to breathing in a constant and controlled manner. The phantom used was the Quasar respiratory motion phantom (Modus Medical Devices, Inc., London, ON, 
Canada). The phantom was first positioned on the CT couch and the Phillips Bellows belt was attached around the body of the phantom and a RADPOS detector was positioned directly on the insert, Figure 4.7. A CT scan was performed while a component of the phantom (an acrylic insert) moved in a specific sinusoidal pattern with an amplitude of 1 $\mathrm{cm}$. As this insert moved the Philips Bellows belt expanded and contracted, recording the pattern of motion. The motion was also recorded simultaneously by the RADPOS system. For comparison with another commercial device, the same procedure was repeated using the Varian Real-time Position Management, RPM, system. This system uses a block with optical markers and a system of cameras to record vertical motion. The block was placed on top of the chest wall platform of the phantom adjacent to a RADPOS detector, as shown in figure 4.7. The same motion pattern was repeated and recorded using both the RPM and RADPOS systems and the measured signals were compared. 
a)

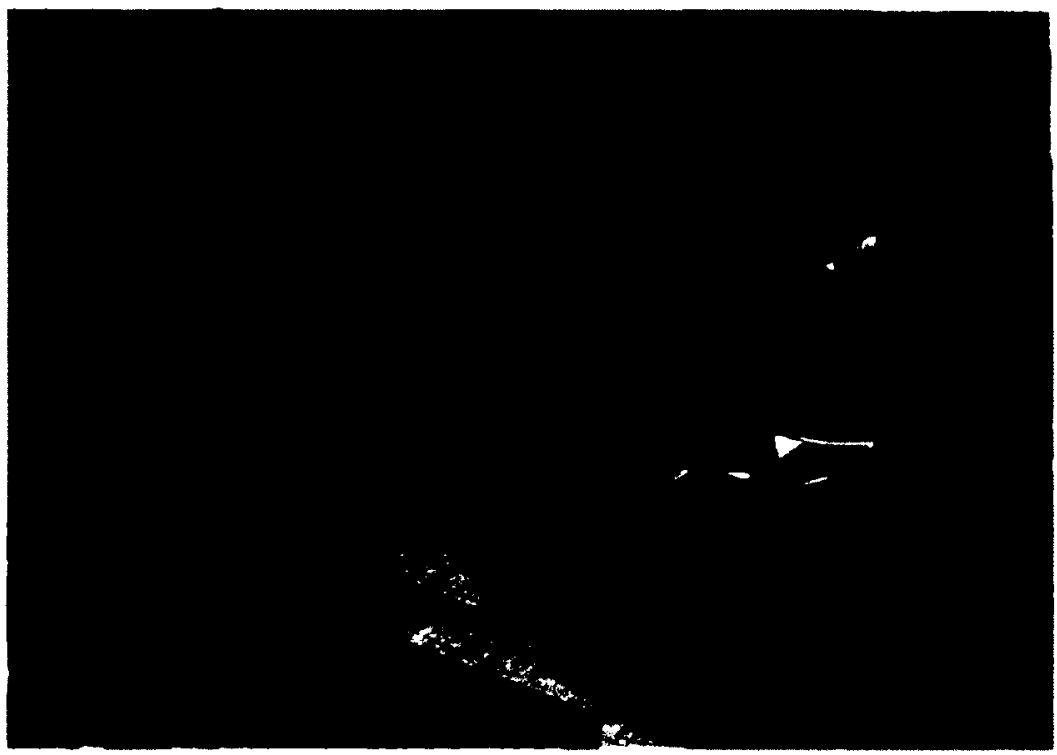

b)

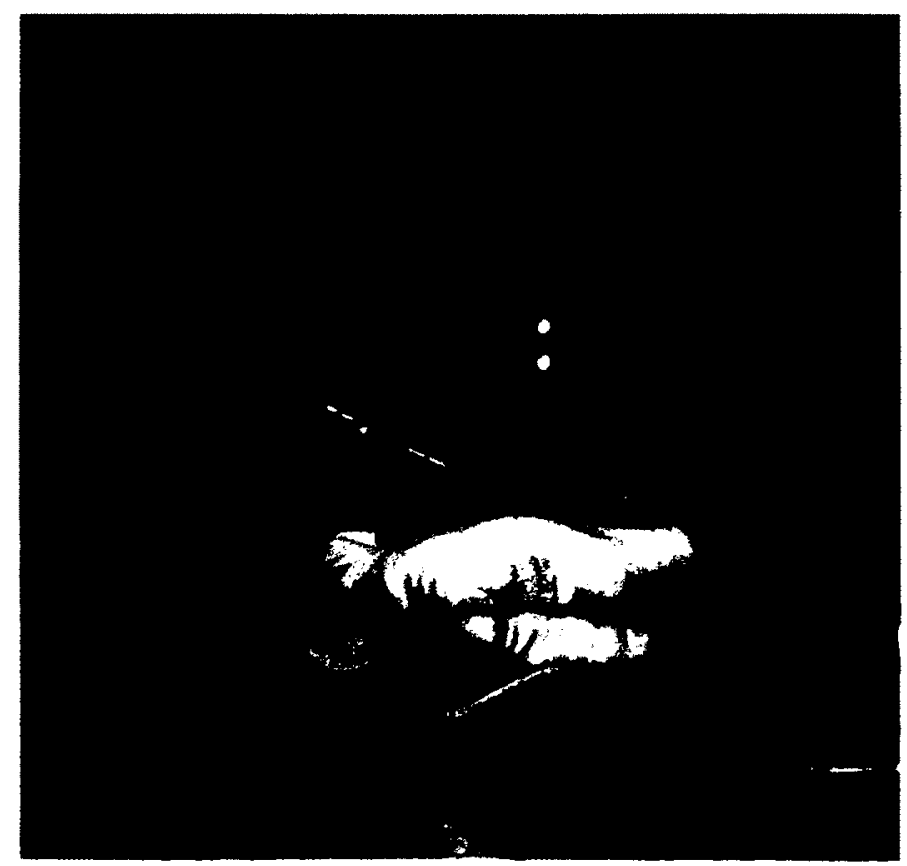

Figure 4.7. Set-up for phantom motion measurements with the RADPOS and a) the Philips Bellows belt and b) the Varian RPM system positioned on top of the Quasar phantom chest wall platform. 
The RADPOS, Philips Bellows Belt and Varian RPM optical marker systems all record displacement with independent timestamps. This meant that steps had to be taken to synchronize the signals from the different systems. At either the beginning or the end of the recording period, the phantom motion was stopped, while the recording continued. This created a flat line signal that occurred at the same point in time regardless of the system used for recording. Once the data were plotted according to the time stamps of each system, the time when the flat line began was identified and the difference between these times from each system, $t_{\text {shift, }}$ was used to shift the time stamps from the RADPOS system, see figure 4.8 .
a) $t_{\text {gatime }}(R A D P O S)-t_{\text {Ratline }}(R P M)=t_{\text {shift }}(R A D P O S)$
b) $t_{i}(R A D P O S)-t_{\text {shif }}(R A D P O S)=i_{i}(R A D P O S)$

Equation 4.1

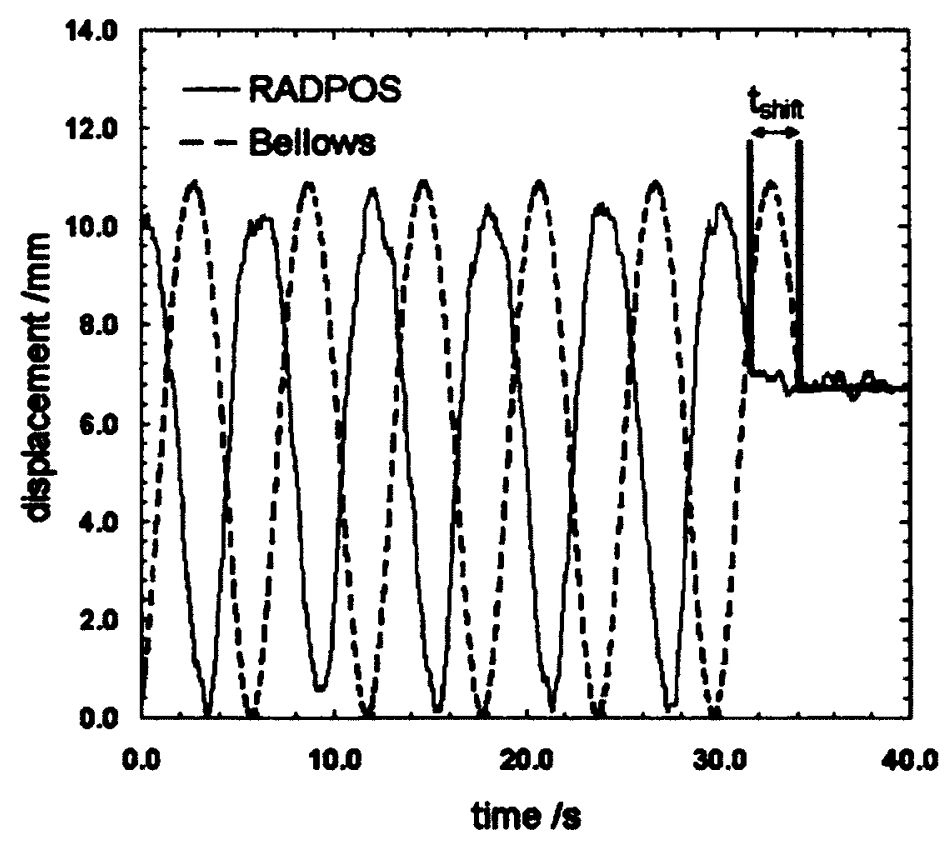

Figure 4.8. Example of how the RADPOS data were shifted to correspond with the RPM data according to the time at which the flat line signal began. 


\subsubsection{Clinical study}

Subjects for this clinical study were recruited from a population of patients scheduled for 4DCT simulation and external beam treatment for histologically-confirmed lung cancer. Candidates were referred by their physicians and eligibility was determined by assessing treatment strategy and schedule. Data were collected for 10 patients, including four males and six females, aged 51-86 years, at time of 4DCT and for an average of 9 treatments (range of 6-15). The diagnoses included four upper lobe (Patients $1,4,5$ and 8) and one lower lobe (Patient 6) right lung/bronchus tumours, one upper lobe (Patient 10) and two lower lobe (Patients 2 and 9) left lung/bronchus tumours, one main bronchus right side tumour (Patient 7) and one thymoma (Patient 3). This project was approved by the Ottawa Hospital Research Ethics Board and all patients gave informed consent before participating in the study. The consent form can be found in Appendix A. Patients did not feel uncomfortable with the measurements and all were willing to continue participation in the study.

Measurements were taken at the time of $4 \mathrm{DCT}$ and again throughout treatment. Each 4DCT scan was performed on a Philips Brilliance Computed Tomography scanner, Koninklijke Philips Electronic N.V. Before the 4DCT, three RADPOS sensors were positioned on the patient's skin aligned in the superior/inferior direction with the dosimeters in the 'bubble-down' orientation. Detector 1 was placed under the Philips Bellows device as this is a point where large displacement can occur. The Philips Bellows device is currently used in this clinic to measure breathing patterns to sort 4DCT data into appropriate breathing phases. Detector 2 was positioned on the skin directly above the tumour. Detector 3 was placed at a reference point $7 \mathrm{~cm}$ inferior to the suprasternal notch (the CT reference isocenter) since this point is well defined within the 
patient and machine geometry. After an initial scout scan, the tumour position was noted to help ensure that the Detector 2 sensor position would be within the treatment field. Any necessary adjustments to the original placement of this detector were made before the scan was continued. A fourth detector was placed on the CT couch as a reference. The transmitter was attached to the couch within the recommended range from the detectors. ${ }^{[47]}$ The geometry of this set-up can be seen in figure 4.9. The three-dimensional position coordinates of the RADPOS sensors as well as abdominal displacement measured by the Philips Bellows device were recorded throughout the scanning process. The points where Detectors 1,2 and 3 had been positioned were then marked with tattoos, so that the detectors could be accurately repositioned during treatments. Since this study aimed to characterize breathing motion from the time of the planning CT until the end of treatment, detector positions had to be chosen before treatment planning occurred and remained consistent throughout treatment. For routine in vivo dosimetry use, detector positions could always be chosen once the treatment plan was completed to ensure dose measurements were taken in specific regions of interest. 


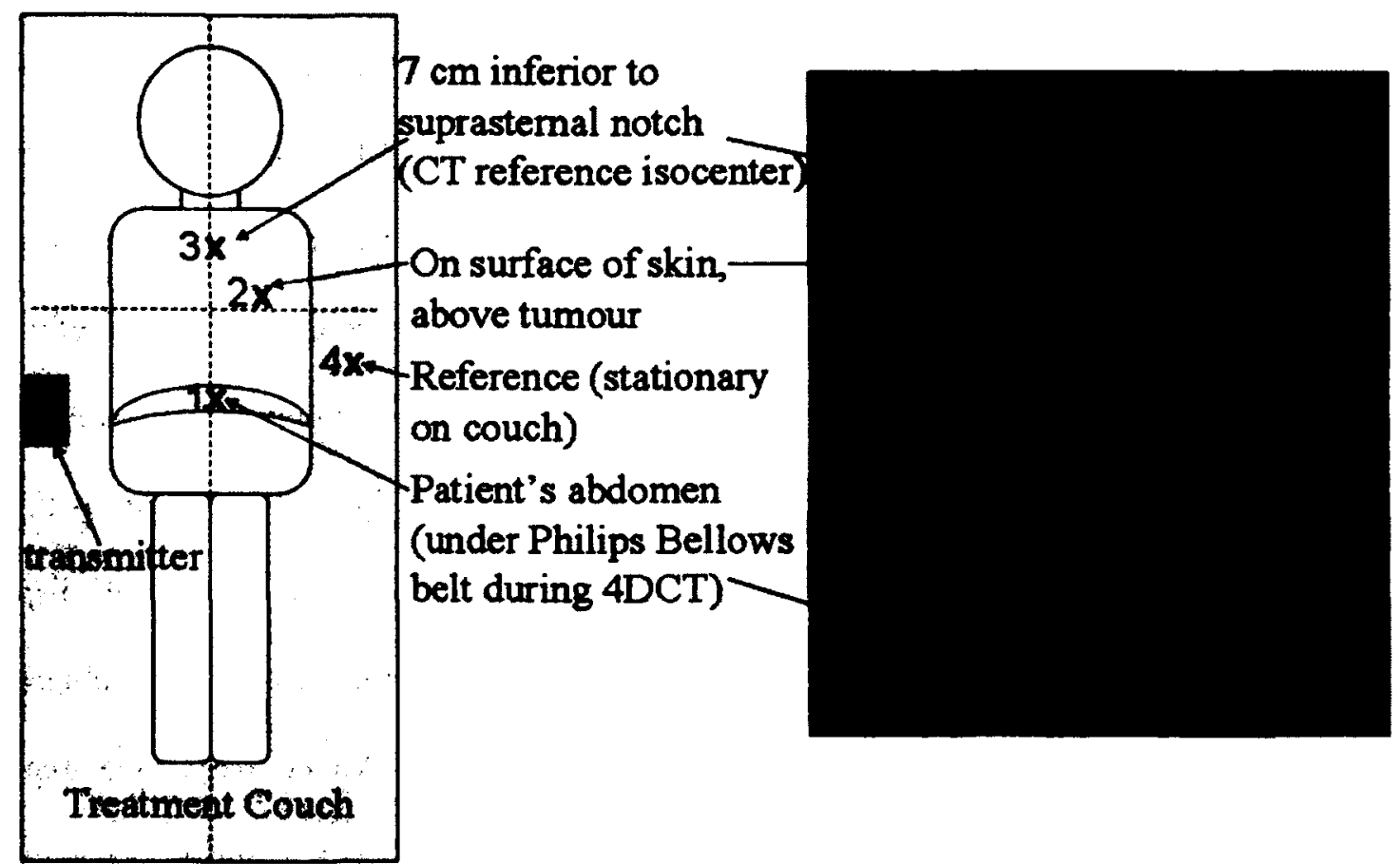

Figure 4.9. Placement of RADPOS detectors during 4DCT and treatment delivery.

The interference effects of the CT bore on the RADPOS-read detector positions have been studied and are described in Chapter $3 .{ }^{[95]}$ Proximity to the CT bore was found to cause a systematic shift in the RADPOS-read positions, the magnitude of which varied with the location of the detector. To measure the patient's breathing pattern with minimal interference, the CT couch was pulled far from the bore once the scans were completed and the displacement of the detectors was recorded for approximately two minutes while the patient breathed normally. For use during treatments, the threshold voltage of the MOSFETs was also read before and two minutes after treatment and the surface dose was calculated using previously determined calibration coefficients. Measurements were repeated as often as possible throughout the course of a patient's treatment. 
For all patients in this study, the largest displacement was measured with Detector 1 (lower abdomen) in the anterior/posterior, AP, direction, which was the $z$ axis of our system. This data set was then used for numerical analysis and day to day comparisons of amplitude and period since the larger amplitude gives a better signal to noise ratio. Position information was however collected from all three detectors on the patient's body, and these data could be useful in other applications or analyses. For each treatment fraction, the daily average amplitude measured by Detector 1 in the anterior/posterior direction, $\hat{z}_{D 1}$, and daily average period, $\hat{T}_{D 1}$, were calculated. Amplitude was determined by finding the inhale and exhale positions for each breathing cycle and calculating the average of all inhale-exhale differences. Period was calculated using the time that inhale and exhale points occurred. Student $t$ tests were used to compare these average values ( $\hat{z}_{D 1}$ and $\hat{T}_{D 1}$ ) for each treatment day with the same measurement made at the time of the 4DCT, $\hat{z}_{D 1,4 D C T}$, to determine if there was significant variation. The null hypothesis of the test was that the data sets were equivalent and an $\alpha$ value of 0.05 (95\% confidence limit) was used as the cut off for significance.

To investigate the extent of trends in breathing motion over time once treatment began, the correlations between the amount of days since the $4 D C T, \Delta t_{4 D C T}$, and the daily average amplitude, $\hat{z}_{D 1}$, and period, $\hat{T}_{D 1}$, as well as the standard deviations of the daily amplitude, $\sigma_{z_{D 1}}$ and period, $\sigma_{T_{D 1}}$ measured during treatment were calculated. The number of measurements taken for each patient is defined as $\boldsymbol{n}$ and $\boldsymbol{r}$ values are reported as $r(n-2)$. While the standard deviation reflects the general daily variation, specific irregularities such as coughing can be seen in the maximum and minimum amplitude, 
$\Delta_{z_{D 1}}$, and period, $\Delta_{T_{D 1}}$ measured each day. These values were also calculated for each patient and treatment day.

To compare dose measurements with the treatment planning system calculations, treatment plans were analyzed to find the location of the RADPOS detectors on the original CT scans. One of the advantages of the RADPOS system is that each detector contains a position sensor that is visible on the CT image. This helped eliminate uncertainties due to inhomogeneities in the anatomy where the location of the detector was questionable by visual inspection alone. Calculation voxel size was $2.5 \times 2.5 \times 2.5 \mathrm{~mm}^{3}$ so in some cases the detector location took up multiple voxels and an average and standard deviation for the calculated dose, $\hat{D}_{T P} \pm \sigma_{D_{T P}}$, was determined.

\subsection{RESULTS \& DISCUSSION}

\subsubsection{Phantom study}

When used to measure simulated breathing motion on a 4D Quasar phantom, the RADPOS system agreed well with the Phillips Bellows and Varian RPM systems, which are currently accepted for clinical use. The correlation between the RADPOS displacements and those measured by the Bellows and RPM systems were 0.95 and 0.99 , respectively. Since the Varian RPM system measures quantitative motion in the vertical direction, direct comparisons could be made with the displacement measurements recorded by the RADPOS system. A Mann-Whitney statistical test was performed and it was found that the RPM measurements were significantly higher than the RADPOS measurements, which reflects the interference seen at the peak of the sinusoidal curves ( $\mathrm{n} 1=\mathrm{n} 2=4000, \mathrm{p}<0.05$ two-tailed). Comparing the absolute values however, it was found that the RADPOS-measured displacements were on average within $0.05 \mathrm{~mm}$ of 
those recorded by the Varian RPM system. The displacement shown in these plots is only in one dimension (z), so the uncertainty on RADPOS measurements within this range is $\pm 1.1 \mathrm{~mm}$. These tests show the potential for the RADPOS system to act as a continuous check of patient motion and as an alternative to external optical markers used during treatment in respiratory gating methods proposed in other work. ${ }^{[9]}$

a)

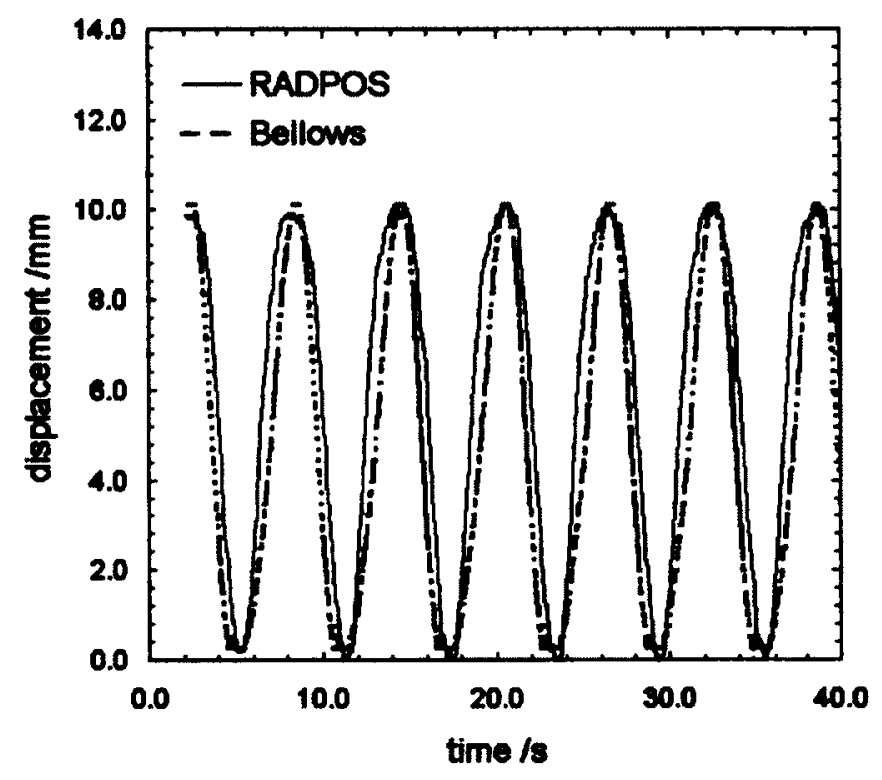


b)

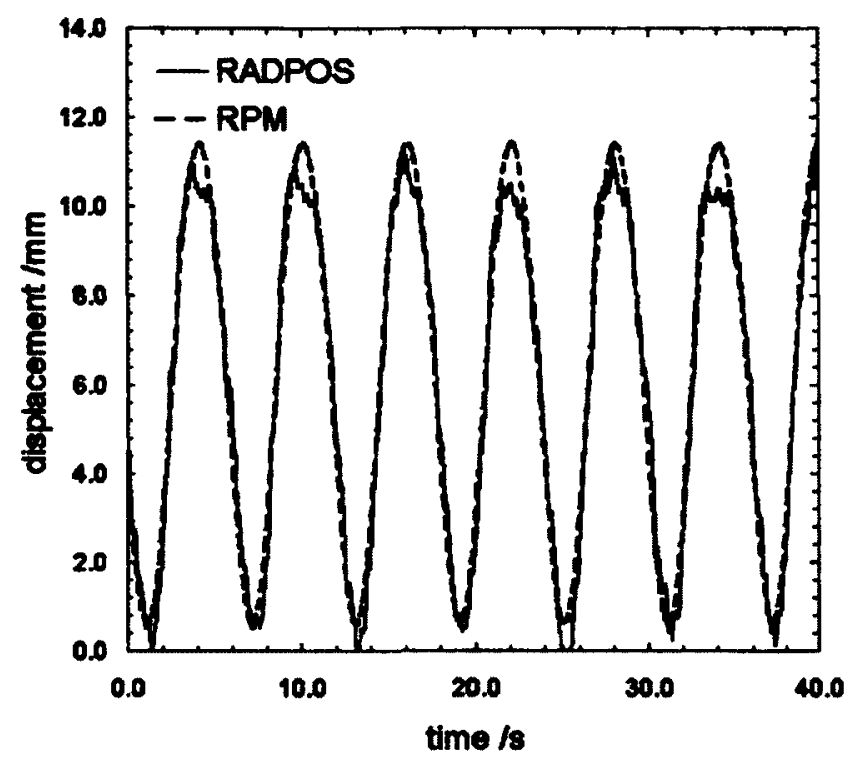

Figure 4.10. Measurements of a 4D phantom's periodic motion by the RADPOS system and a) Phillips Bellows and b) Varian RPM with synchronized time.

\subsubsection{Clinical study}

\subsubsection{Clinical experience}

Daily set-up of the equipment was completed quickly and did not interfere with regular patient scheduling. On the first day of treatment the gantry was rotated a full $360^{\circ}$ with the transmitter in place before treatment began to ensure that a collision would not occur during cone-beam CT or treatment. The placement of the transmitter was determined by the tumour and detector locations, treatment fields, as well as patient size. No interventions due to patient movement or changes in breathing pattern were made during these patient treatments as this was only a preliminary clinical study; however, in future scenarios tolerance limits for motion could be defined and easily monitored outside of the treatment room using the RADPOS detectors and graphical display. 


\subsubsection{Position measurements}

Inter-fraction variations

The average amplitude for the ten patients over the course of treatment ranged from $(4.8 \pm 2.3)$ to $(8.9 \pm 1.4) \mathrm{mm}$. Absolute values of measured amplitude are difficult to compare between studies done in different clinics due to varying instruments, measurement techniques, and most importantly detector placements. Speed or period of breathing is however much easier to relate across different studies. The average period over all measurement days ranged from $(2.6 \pm 0.9)$ to $(3.6 \pm 1.2) \mathrm{s}$ for the ten patients in this study and the average period over all measurements and patients was $(3.2 \pm 1.6) \mathrm{s}$. These values are consistent with similar work, for example a study that followed ten patients throughout treatment using optical markers. ${ }^{[97}$ The range of patient-specific breathing periods found was $(1.88 \pm 0.82)$ to $(4.38 \pm 0.41) \mathrm{s}$, with an overall average of $(3.71 \pm 1.03)$ s. $^{[97]}$

The maximum day-to-day variations in average amplitude and period for each patient ranged from $\left(\hat{z}_{D 1 \max }-\hat{z}_{D 1 \min }\right)=1.4$ to $8.6 \mathrm{~mm}$ and $\left(\hat{T}_{D 1 \max }-\hat{T}_{D 1 \min }\right)=0.2$ to $1.8 \mathrm{~s}$. Daily variations were patient-specific, as some patients displayed large differences between breathing patterns measured during the 4DCT and treatment, while others displayed no consistent trend. Results from the Student $\mathrm{t}$ tests comparing amplitude and period measured during treatment to those measured at the time of the 4DCT are presented in table 4.1. For the majority of treatment fractions, amplitude measurements differed significantly from measurements made at the time of the planning CT for six of ten patients. This number increased to nine of ten patients when the period of breathing was compared. A possible reason for this might be the use of the Phillips Bellows belt 
during the 4DCT, which could restrict anatomy and change the nature of breathing motion. Healthy volunteers did not however report any restrictions to breathing caused by the Bellows belt. Another factor could be elevated anxiety levels due to the unfamiliar environment or the increased time period between the 4DCT and the start of treatment, which was an average of 13 days with a range of 8-21 days, compared to the time between daily fractions. Once treatment began, inter-fraction variations decreased as breathing patterns appeared to be more consistent from day to day. Figure 4.11 shows statistical plots of the amplitude and period data which display the measurement at time of the 4DCT, the treatment-averaged value, along with the $75^{\text {th }}$ and $25^{\text {th }}$ percentile of measurements made during treatment for each patient. 
Table 4.1. Results from Student $t$ test comparing average amplitude and period measured during treatment to values measured at the time of the $4 D C T$. Results with a p-value less than $\alpha=0.05$ are considered significant and are shown in bold.

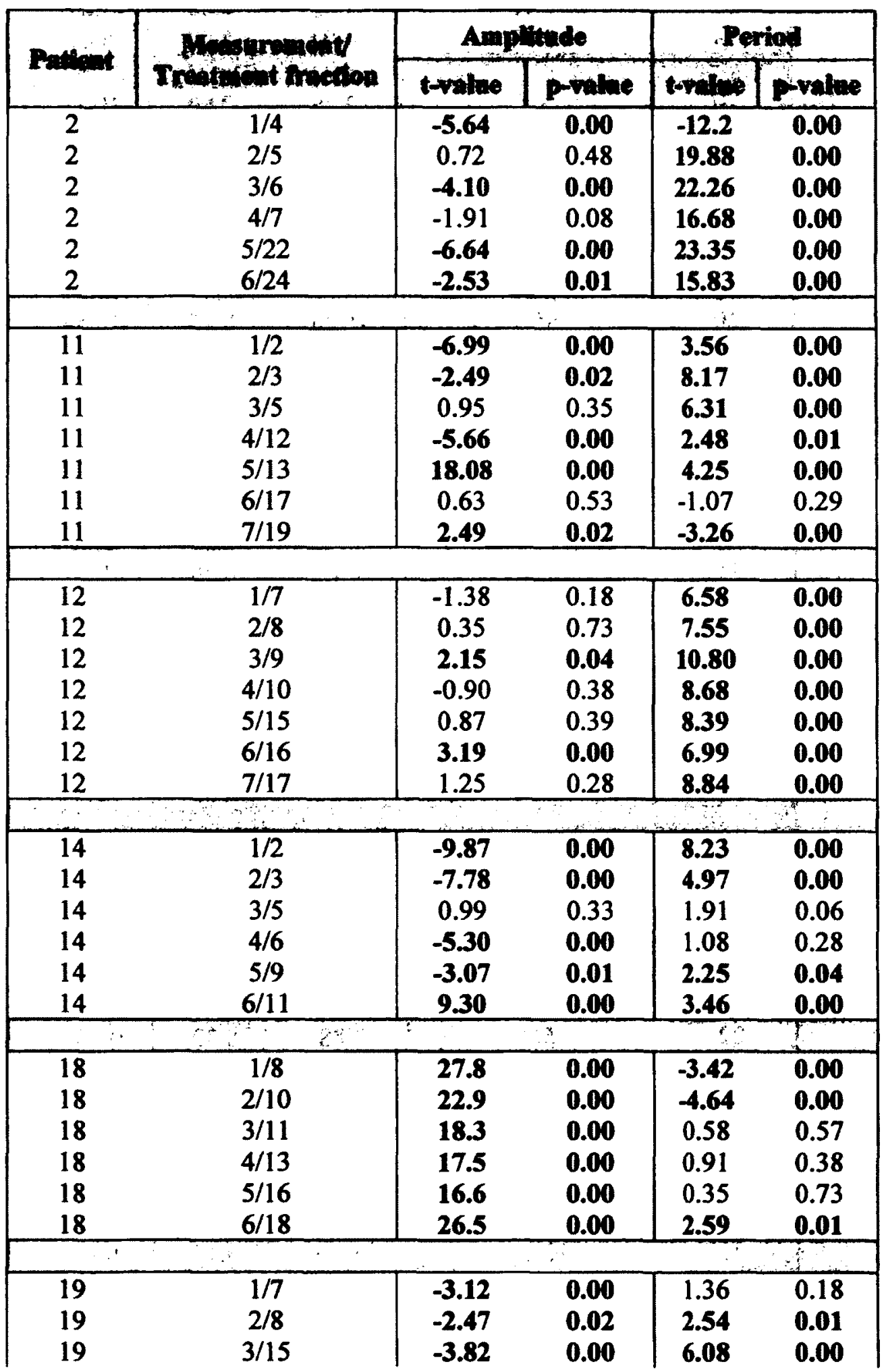




\begin{tabular}{|c|c|c|c|c|c|}
\hline $\begin{array}{l}19 \\
19 \\
19 \\
19 \\
19 \\
\end{array}$ & $\begin{array}{l}4 / 22 \\
5 / 23 \\
6 / 28 \\
7 / 30 \\
8 / 31 \\
\end{array}$ & $\begin{array}{c}-2.75 \\
3.71 \\
-3.72 \\
-3.35 \\
-4.02 \\
\end{array}$ & $\begin{array}{l}0.01 \\
0.00 \\
0.00 \\
0.00 \\
0.00 \\
\end{array}$ & $\begin{array}{c}2.54 \\
7.98 \\
-2.20 \\
-5.67 \\
-6.47 \\
\end{array}$ & $\begin{array}{l}0.01 \\
0.00 \\
0.03 \\
0.00 \\
0.00 \\
\end{array}$ \\
\hline $\begin{array}{l}20 \\
20 \\
20 \\
20 \\
20 \\
20 \\
20 \\
20 \\
20 \\
20 \\
20 \\
20 \\
20\end{array}$ & $\begin{array}{c}1 / 5 \\
2 / 6 \\
3 / 7 \\
4 / 22 \\
5 / 23 \\
6 / 24 \\
7 / 26 \\
8 / 27 \\
9 / 28 \\
10 / 30 \\
11 / 31 \\
12 / 32 \\
13 / 33\end{array}$ & $\begin{array}{c}-3.55 \\
-1.96 \\
-2.03 \\
0.64 \\
-1.09 \\
-0.97 \\
1.06 \\
-1.20 \\
0.00 \\
2.21 \\
0.90 \\
-0.38 \\
-1.17\end{array}$ & $\begin{array}{l}0.00 \\
0.07 \\
0.06 \\
0.53 \\
0.29 \\
0.35 \\
0.31 \\
0.25 \\
1.00 \\
0.04 \\
0.38 \\
0.71 \\
0.26\end{array}$ & $\begin{array}{l}-10.12 \\
-10.08 \\
-10.24 \\
-11.72 \\
-11.95 \\
-11.49 \\
-11.07 \\
-11.80 \\
-11.97 \\
-12.12 \\
-10.92 \\
-12.74 \\
-12.61\end{array}$ & $\begin{array}{l}0.00 \\
0.00 \\
0.00 \\
0.00 \\
0.00 \\
0.00 \\
0.00 \\
0.00 \\
0.00 \\
0.00 \\
0.00 \\
0.00 \\
0.00\end{array}$ \\
\hline $\begin{array}{l}21 \\
21 \\
21 \\
21 \\
21 \\
21 \\
21 \\
21 \\
21\end{array}$ & $\begin{array}{c}1 / 1 \\
2 / 2 \\
3 / 4 \\
4 / 5 \\
5 / 6 \\
6 / 7 \\
7 / 8 \\
8 / 10 \\
9 / 11\end{array}$ & $\begin{array}{l}.11 \\
1.15 \\
2.50 \\
2.66 \\
0.89 \\
2.71 \\
1.66 \\
2.01 \\
0.48\end{array}$ & $\begin{array}{l}0.04 \\
0.26 \\
0.02 \\
0.01 \\
0.38 \\
0.01 \\
0.11 \\
0.05 \\
0.63\end{array}$ & $\begin{array}{c}-2.46 \\
-4.67 \\
1.01 \\
-1.29 \\
1.29 \\
-3.03 \\
-3.63 \\
-7.66 \\
-5.45\end{array}$ & $\begin{array}{l}0.02 \\
0.00 \\
0.31 \\
0.20 \\
0.20 \\
0.00 \\
0.00 \\
0.00 \\
0.00\end{array}$ \\
\hline$\ldots$ & 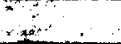 & $\therefore$ & & & $\because$ \\
\hline 22 & $1 / 2$ & -0.54 & 0.59 & 1.04 & 0.30 \\
\hline 22 & $2 / 3$ & 2.76 & 0.01 & 1.31 & 0.20 \\
\hline 22 & $3 / 4$ & 0.11 & 0.91 & 0.27 & 0.79 \\
\hline 22 & $4 / 5$ & -1 & 0.33 & -1.65 & 0.11 \\
\hline 22 & $5 / 6$ & 0.84 & 0.41 & -0.61 & 0.55 \\
\hline 22 & $6 / 7$ & 0.88 & 0.39 & 0.60 & 0.55 \\
\hline 22 & $7 / 8$ & 1.25 & 0.22 & -0.52 & 0.61 \\
\hline 22 & $8 / 9$ & 2.12 & 0.04 & -1.91 & 0.06 \\
\hline 22 & $9 / 10$ & 1.12 & 0.27 & -1.26 & 0.21 \\
\hline 22 & $10 / 14$ & -4.14 & 0.00 & 0.97 & 0.34 \\
\hline 22 & $11 / 15$ & -4.16 & 0.00 & 2.10 & 0.04 \\
\hline 22 & $12 / 16$ & -1.49 & 0.15 & -0.95 & 0.35 \\
\hline 22 & $13 / 18$ & 0.85 & 0.40 & -2.55 & 0.01 \\
\hline 22 & $14 / 19$ & -1.75 & 0.09 & -2.39 & 0.02 \\
\hline
\end{tabular}




\begin{tabular}{|c|c|c|c|c|c|}
\hline 22 & $15 / 20$ & -13.9 & 0.00 & -2.32 & 0.02 \\
\hline 24 & $1 / 1$ & -3.79 & 0.00 & -3.59 & 0.00 \\
\hline 24 & $2 / 4$ & -4.19 & 0.00 & -3.48 & 0.00 \\
\hline 24 & $3 / 16$ & -3.19 & 0.01 & -3.64 & 0.00 \\
\hline 24 & $4 / 18$ & -5.96 & 0.00 & -5.00 & 0.00 \\
\hline 24 & $5 / 19$ & 1.51 & 0.15 & -4.69 & 0.00 \\
\hline 24 & $6 / 21$ & -1.3 & 0.22 & -6.81 & 0.00 \\
\hline 24 & $7 / 22$ & -2.11 & 0.05 & -7.70 & 0.00 \\
\hline 24 & $8 / 23$ & -4.5 & 0.00 & -6.24 & 0.00 \\
\hline 24 & $9 / 24$ & -0.57 & 0.57 & -5.36 & 0.00 \\
\hline 24 & $10 / 26$ & -2.74 & 0.02 & -5.65 & 0.00 \\
\hline 24 & $11 / 27$ & -6.54 & 0.00 & -6.00 & 0.00 \\
\hline 24 & $12 / 29$ & -2.56 & 0.02 & -5.05 & 0.00 \\
\hline 24 & $13 / 32$ & 2.59 & 0.02 & -5.46 & 0.00 \\
\hline 24 & $14 / 33$ & -6.77 & 0.00 & -7.38 & 0.00 \\
\hline
\end{tabular}


a)

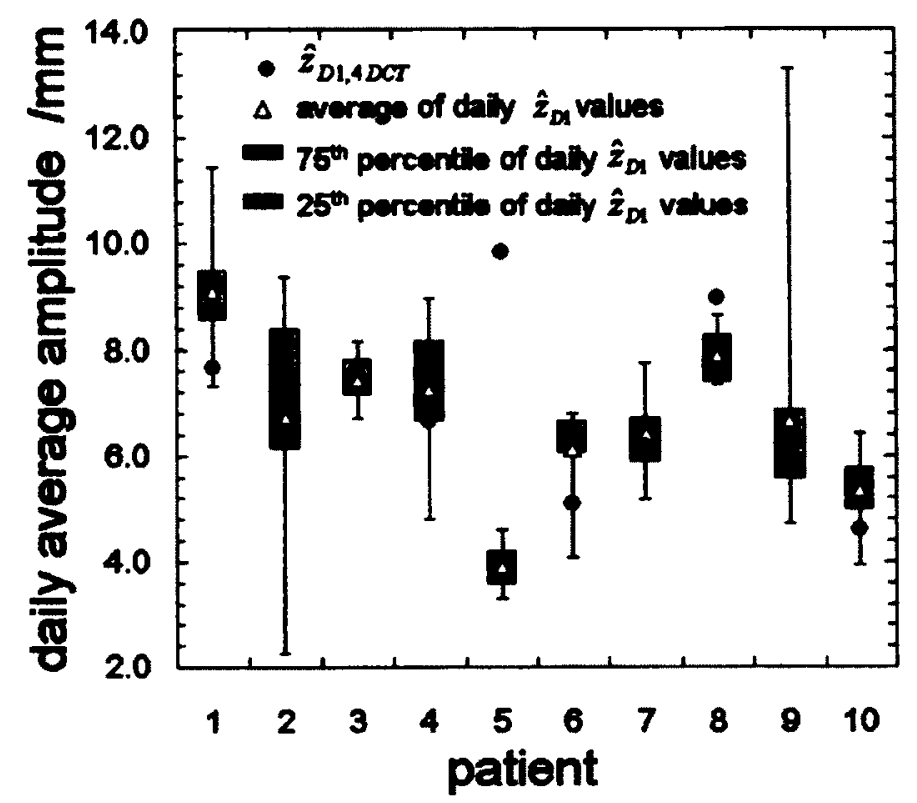

b)

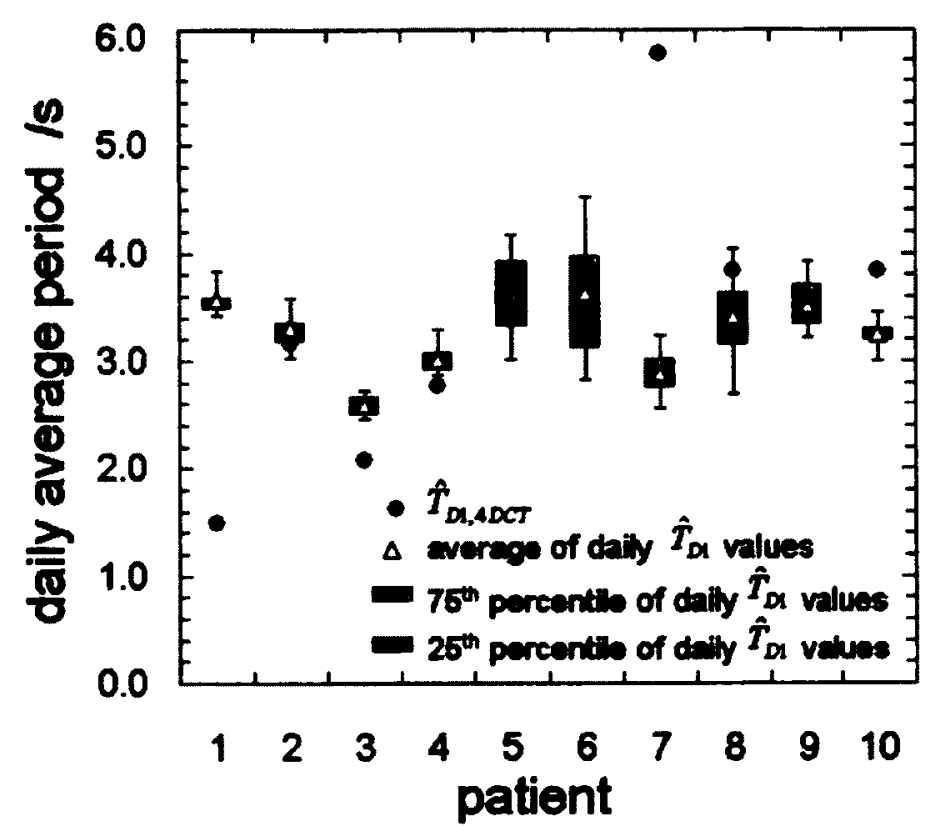

Figure 4.11. Statistical plot of the daily average a) amplitude in the anterior/posterior direction, $\hat{z}_{D 1}$, and b) period, $\hat{T}_{D 1}$, measured by Detector 1 during treatment fractions compared to the same measurement taken during the $4 D C T\left(\hat{z}_{D 1,4 D C T}\right.$ and $\left.\hat{T}_{D 1,4 D C T}\right)$. 
The fractions when the amplitude of breathing motion is greater during treatment than during the planning $\mathrm{CT}$ indicate times when the tumour could potentially move partially out of the treatment field. If a patient breathes more deeply than was originally anticipated during planning, there is potential for a partial geographic miss since the tumour could move through a volume greater than that encompassed by the treatment margins. When the tumour moves less than during the 4DCT $\left(\hat{z}_{D 1}<\hat{z}_{D 1,4 D C T}\right)$, healthy tissue could receive more dose than expected, leading to compromised pulmonary function or other health side effects.

Results of the correlation calculations between the amount of days since the $4 D C T, \Delta t_{4 D C T}$, and the daily average amplitude, $\hat{z}_{D 1}$, period, $\hat{T}_{D 1}$, and standard deviations, $\sigma_{z_{D 1}}$ and $\sigma_{T_{D 1}}$, measured during treatment are shown in table 4.. Calculations were compared to the critical $r$ values for the specific degrees of freedom at a confidence limit of $90 \%(\alpha=0.1)$, and values that were found to be significant are shown in bold. One common result was the significant negative correlation between period and days since planning CT found for six patients, Patients $2,4,6,7,9$ and 10 . This trend indicates a tendency for the rate of breathing to increase as time goes on. 
Table 4.2. Results of the correlation analysis between the number of days since a patient's $4 D C T, \triangle t_{4 D C T}$, and daily average amplitude, $\hat{z}_{D 1}$, daily average period, $\hat{T}_{D 1}$, and standard deviations of these values, $\sigma_{z_{D 1}}$ and $\sigma_{T_{D 1}}$. Moderate to very strong correlations $(0.4<|r|>1.0)$ are show in bold.

\begin{tabular}{|c|c|c|c|c|c|}
\hline \multirow{3}{*}{ Patient } & \multirow{2}{*}{$\begin{array}{c}\text { Number of } \\
\text { measurements }\end{array}$} & \multicolumn{4}{|c|}{$\begin{array}{c}\text { Correlation coefficient } \\
r\end{array}$} \\
\cline { 3 - 6 } & & $\hat{z}_{D 1}$ & $\hat{T}_{D 1}$ & $\sigma_{z_{D 1}}$ & $\sigma_{T_{D 1}}$ \\
\hline 1 & 6 & -0.1 & -0.5 & 0.3 & 0.4 \\
\hline 2 & 6 & -0.5 & -0.9 & 0.3 & 0.2 \\
\hline 3 & 7 & -0.6 & 0.2 & 0.1 & 0.5 \\
\hline 4 & 6 & -0.8 & -0.7 & -0.9 & -0.7 \\
\hline 5 & 6 & -0.1 & 0.8 & 0.2 & 0.7 \\
\hline 6 & 8 & 0.1 & -0.6 & 0.0 & -0.4 \\
\hline 7 & 13 & -0.7 & -0.9 & -0.1 & -0.2 \\
\hline 8 & 9 & 0.3 & -0.5 & 0.9 & 0.0 \\
\hline 9 & 15 & 0.6 & -0.5 & -0.3 & 0.3 \\
\hline 10 & 14 & -0.3 & -0.6 & 0.4 & -0.6 \\
\hline
\end{tabular}

It was originally hypothesized that breathing may slow down over time due to patient relaxation; however this has not been found to be the case. These findings agree with an earlier study by a group that measured breathing motion characteristics of lung cancer patients at the beginning of treatment and again within one week of the last treatment. All nine patients enrolled in that study had significant increases in both frequency and amplitude of respiration based on spirometry measurements and in two patients the vital capacity increased by more than $30 \%{ }^{[98]}$

Intra-fraction variations

Intra-fraction variations in breathing patterns, measured by the standard deviations of the daily amplitude, $\sigma_{z_{D 1}}$ and period, $\sigma_{T_{D 1}}$, were also found to be very patient-specific. The correlation of these values with the amount of days since a patient's 4DCT, $\Delta_{4 D C T}$, was calculated to investigate whether there were any patterns in the consistency of patients' breathing motion as treatment progressed (table 4.2). While the 
standard deviation reflects the general daily variation, specific irregularities such as coughing can be seen in the maximum and minimum amplitude, $\Delta_{z_{D 1}}$, and period, $\Delta_{T_{D 1}}$, measured each day. Statistical plots of these values are displayed in figure 4.12. Variations of $\Delta_{z_{D 1}}=5.6$ to $24.1 \mathrm{~mm}$ and $\Delta_{T_{D 1}}=2.7$ to $11.9 \mathrm{~s}$ were seen during single measurement days with Patient 6 having the largest irregularities in both amplitude and period. These values correspond to differences of 93 to $364 \%$ and 79 to $263 \%$ of the daily average amplitude and period, respectively. Patient 6 was the sole patient with a lower lobe right bronchus/lung tumour.

a)

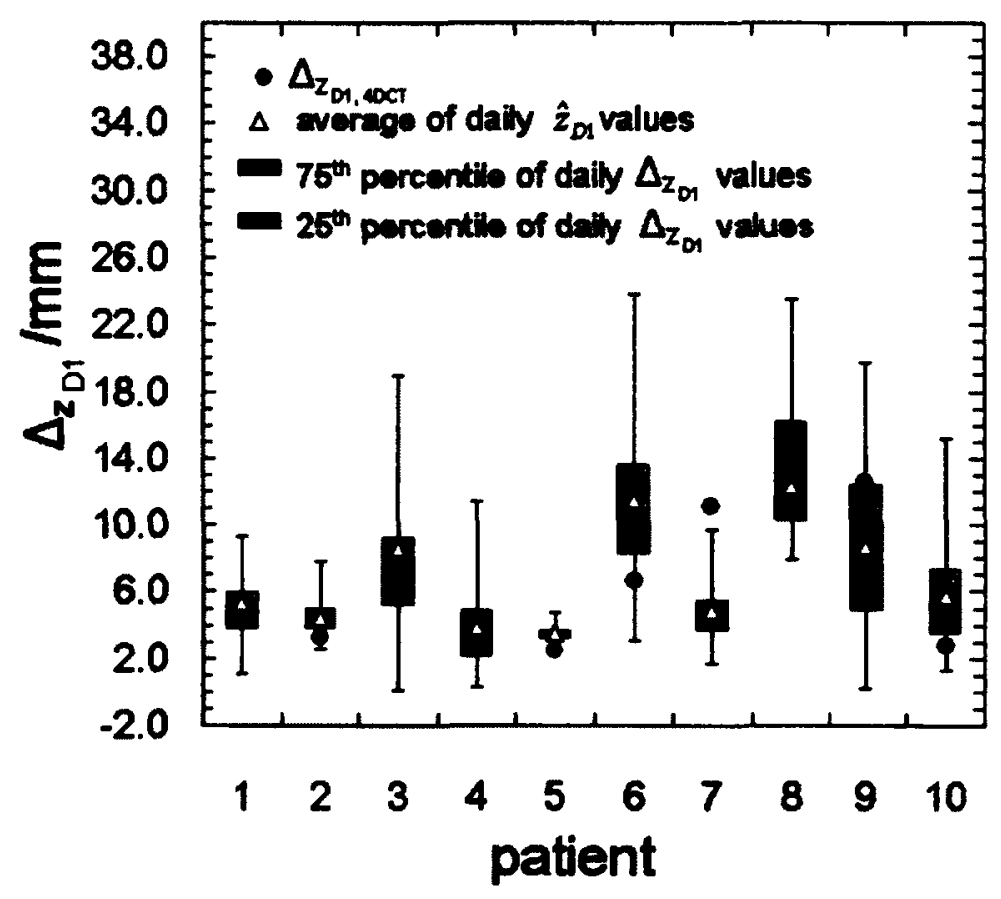


b)

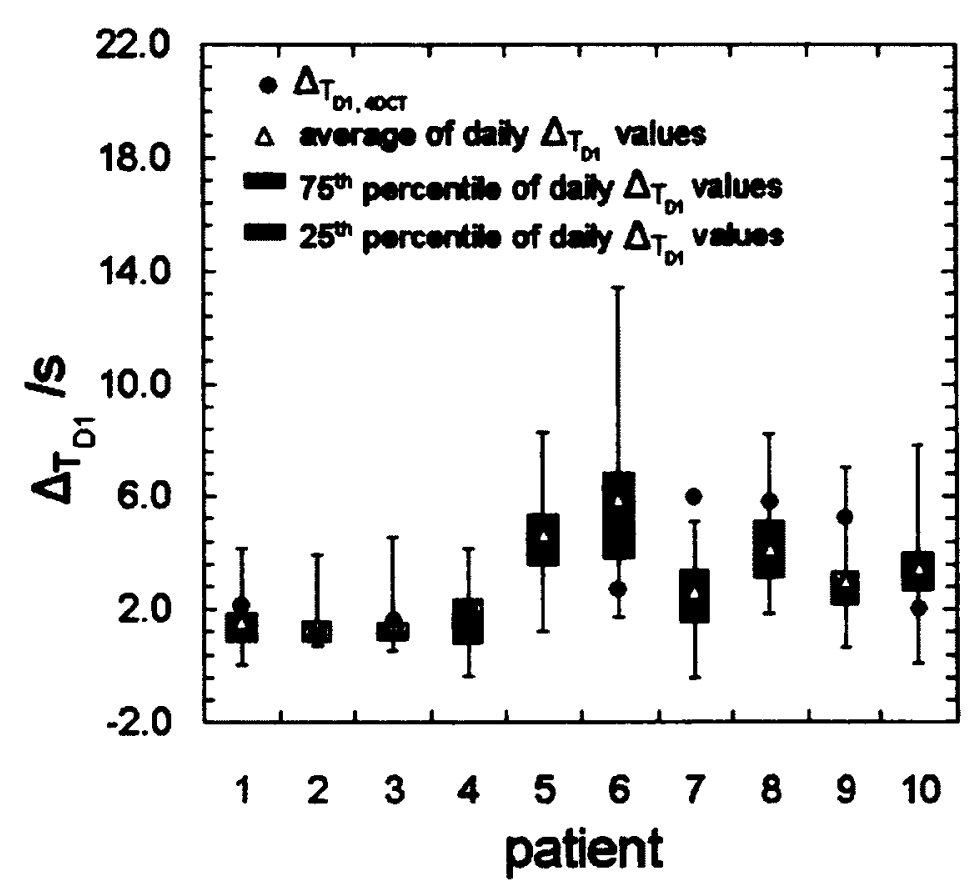

Figure 4.12. Statisical plot of a) $\Delta_{z_{D 1}}=$ (daily max amplitude - daily min amplitude) and b) $\Delta_{T_{D 1}}=$ (daily $\max$ period - daily min period) measured Detector 1 during treatment fractions compared to the same measurement taken during the $4 \mathrm{DCT}\left(\Delta_{z_{D 1}, 4 D C T}, \Delta_{T_{D 1}, 4 D C T}\right)$.

A study by Juhler-Nottrup et al., 2007, looked at intra- and inter-fraction variations in breathing patterns using external optical markers placed on the bony part of the chest wall. ${ }^{[89]}$ Measurements were made on eleven patients during thirty treatment fractions and it was found that the mean amplitude measured during one session corresponded on average to less than half of the full motion extent throughout the course of treatment. It was therefore concluded that margin reductions based on one planning session are unsafe and respiratory-induced tumour motion measured at the time of a planning 4DCT should be considered as only an estimation of motion expected during treatment. ${ }^{[79,89]}$ Opposite conclusions have been drawn by Bosmans et al., 2006 and 
Redmond et al., 2009 who both investigated tumour excursion using multiple CT scans throughout the course of treatment. ${ }^{[99,100]}$ Gross-tumour-volume excursions were found to be stable between all scans so it was concluded that tumour motion at the time of simulation is a valid predictor of excursion later in the treatment. ${ }^{[99,100]}$

Based on the frequent deviations from amplitude and period measured during the planning 4DCT, we must agree with conclusions by Juhler-Nottrup et al, $2007 .^{[89]}$ To this end, the RADPOS system can be used throughout treatment to monitor breathing patterns and if large deviations are noted, a second planning CT could be done to reevaluate tumour margins as treatment progresses. The RADPOS system can also be used to provide feedback to the patient to breathe consistently with the breathing patterns measured during the 4DCT.

Cross-correlation analysis

A cross-correlation analysis was done to quantify the similarity of the displacements in the anterior/posterior direction measured simultaneously at the three locations on each patient's body (Detector 1: lower abdomen, originally underneath the Philips Bellows belt, Detector 2: above the patients' tumour, and Detector 3: CT reference isocenter) and to study how this relationship changes over time. To account for differences in phase between data collected at two different locations, one of the datasets was shifted in steps of $0.05 \mathrm{~s}$, and a correlation coefficient, $\rho(i)$, was calculated for each step. The maximum correlation coefficient, $\rho_{\max }$, was determined and the corresponding shift or 'lag time', $i_{\max }$, was noted. The calculation algorithm was written using Matlab code and verified using the package's standard cross correlation function ' $x$ cov'. Killoran et al., 2008, performed a similar analysis of data collected from the same number of 
patients by two infrared detectors on the patients' skin, one at the xyphoid (similar to Detector 1 in this work) and one near the treatment isocenter (similar to Detector 3). ${ }^{[101]}$ In the work presented here, the additional detector placed over the tumour, Detector 2, provides another breathing measurement at a point in the center of the treatment field, where the largest skin dose will be delivered and measured. For each measurement day, there are three correlation coefficients that must be considered:

- Detectors 1 and 2: $\rho_{12}$

- Detectors 1 and 3: $\rho_{13}$

- Detectors 2 and 3: $\rho_{23}$

It is important to note that while the position of Detectors 1 and 3 were similar for each patient, the position of Detector 2 was determined by the individual tumour location and therefore varied from patient to patient, as displayed in figure 4.13. In all cases Detectors 1 and 3 were located along the central axis, or midline, of the patient and in all but one patient, Patient 3, Detector 2 was off-axis. The results, averaged over all measurements and patients, are shown in figure 4.14. 
a)

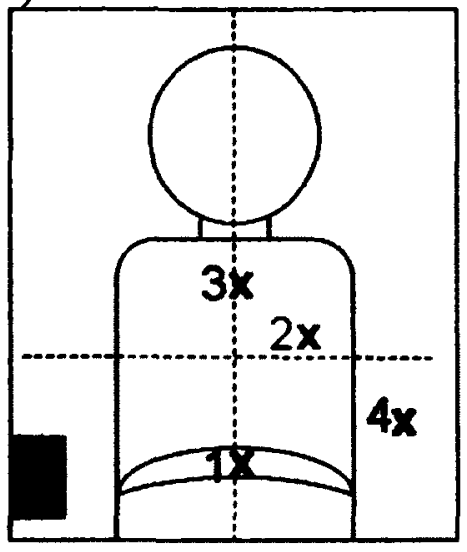

b)

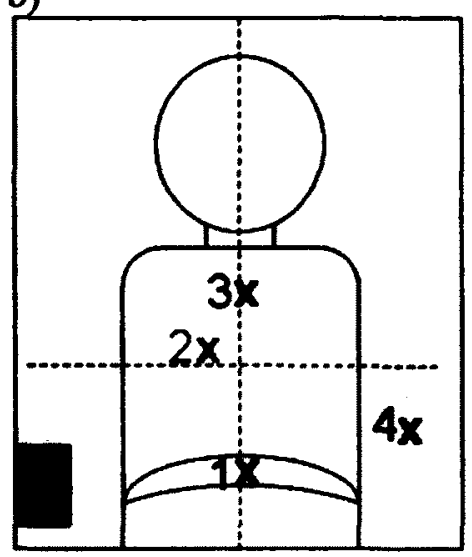

c)

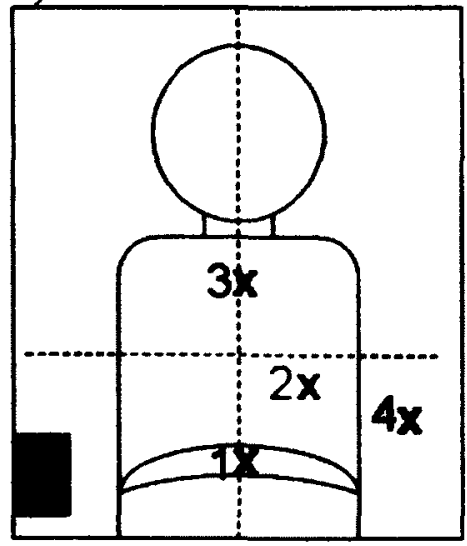

Figure 4.13. Example of detector placement on patients with different tumour locations including a) upper lobe left lung/bronchus tumour b) upper lobe right lung/bronchus tumour $c$ ) lower lobe left lung/bronchus tumour.

a)

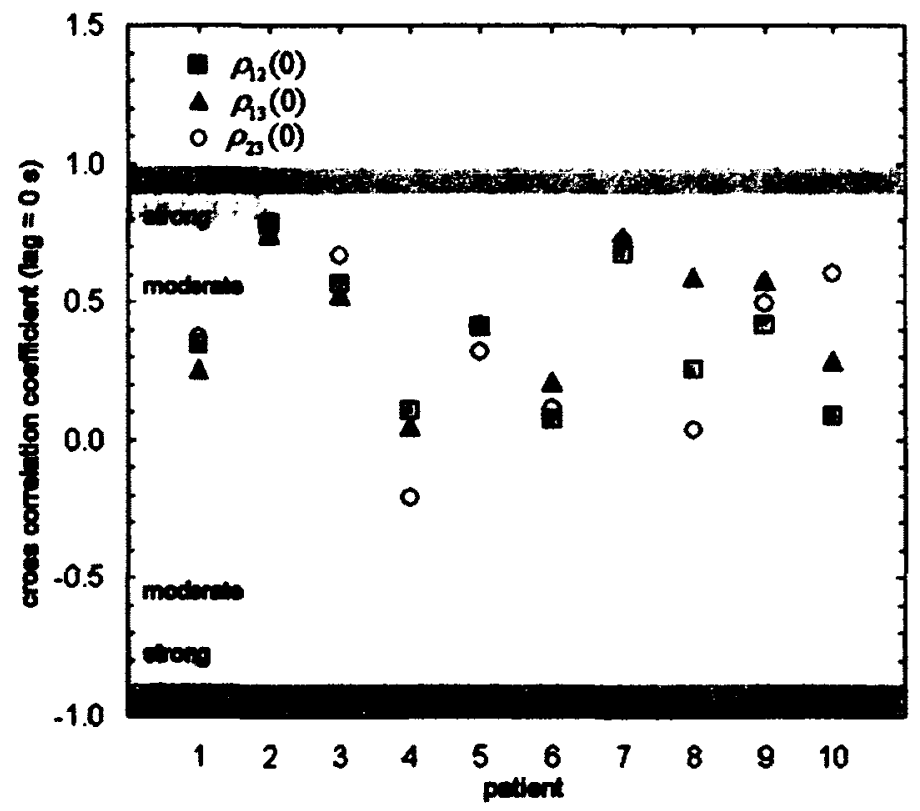


b)

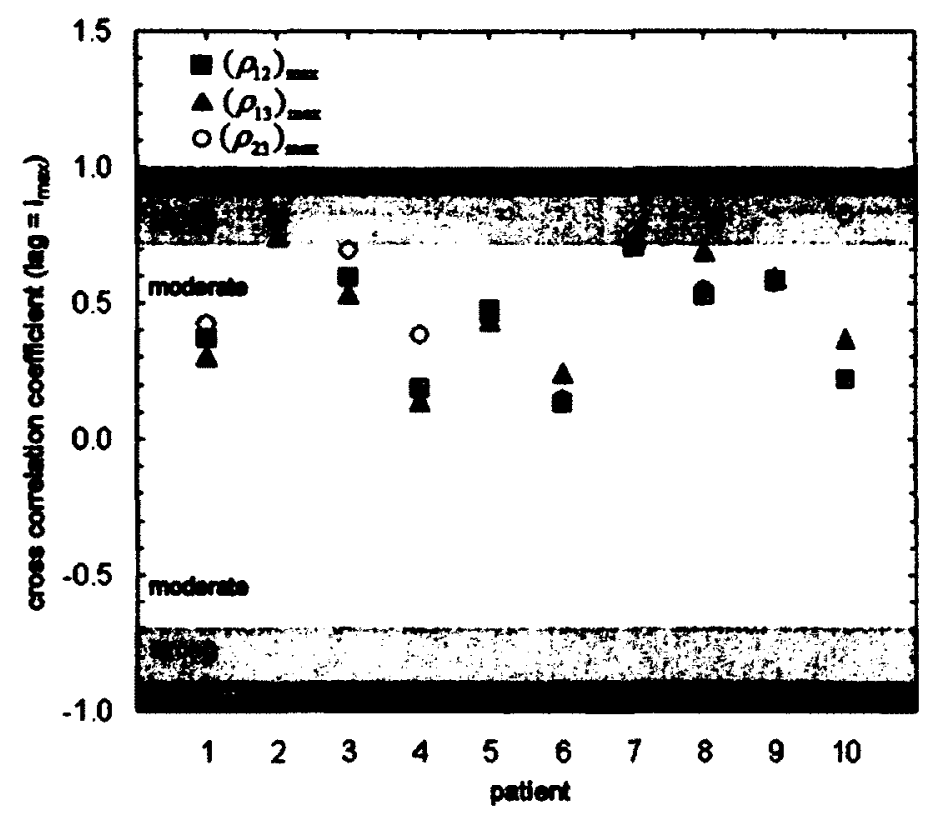

Figure 4.14. Results of the cross correlation analysis of data collected simultaneously by the three detectors on each patient. Figure a) shows the results with zero lag, $\rho(0)$, while b) shows the maximum cross correlation coefficient, $\rho_{\max }$ for each data pair.

Several patterns can be identified by comparing the treatment-averaged correlation coefficients for individual patients. For seven patients, $\rho_{12}(0), \rho_{13}(0)$ and $\rho_{23}(0)$ were all within \pm 0.08 of each other for that specific patient. This means that with zero time shifts, the displacements of the three detectors were either uniformly well correlated or poorly correlated for each of these patients. Once differences in phase were accounted for by shifting the data and finding $\rho_{\max }$, eight patients fell into this category. Only two of these patients (Patients 2 and 7) had a strong correlation between all three detectors, while four patients (Patients 3, 5, 8 and 9) had all moderate correlations. The remaining two patients in this category (Patients 1 and 6) had very weak to weak 
correlations. No patterns concerning tumour location/placement of Detector 2 and correlation results were noted.

The magnitude of the lag or time-shift needed to find $\rho_{\max }$ indicates the extent to which the breathing patterns measured at two locations are out of phase. Since Detectors 2 and 3 were typically located on the patient's upper chest within close proximity to each other, it was expected that the motion measured by these two detectors would be more closely linked to each other than with Detector 1 . Although this was not seen in the magnitude of the correlation coefficients, all but one patient, Patient 1 , had an average $\left(i_{23}\right)_{\max }$ that was 3 to $18 \%$ smaller than the average $\left(i_{13}\right)_{\max }$ or $\left(i_{12}\right)_{\max }$. This indicates that while the displacements of Detectors 1 and 3 might be slightly or significantly better correlated with each other than with Detector 2, the measured breathing patterns are more out of phase, possibly due to the distance between the detectors or to other patient movement not related to breathing. 
Link between external surface motion and internal target motion

Many studies have been dedicated to the correlation between external markers and internal target motion. ${ }^{[6,86-88,102,103]}$ It has been demonstrated that anterior-posterior internal motion is most strongly correlated with the motion of a detector placed on the upper chest. ${ }^{[104]}$ Kini et al. 2001 found that chest wall movement could be used to predict tumour motion to within $3 \mathrm{~mm}$ when compared to fluoroscopic movies and Vedam et al. 2001, and 2003 used similar methods to predict diaphragm motion from external measurements using Varian's RPM system. ${ }^{[56,86,87]}$ External motion measurements must however be used with a clear understanding of their limitations. The wide range of findings on this topic suggest that one cannot necessarily assume external excursion equals internal excursion; however external motion measurements can provide a means of detecting changes in overall breathing patterns and possible deviations from data collected at the time of a planning $C T .^{[56,88,89,91,93,105]}$

A limitation in the use of surrogates is that phase offsets between the motion measured at different points have been found to change over the course of treatment. ${ }^{[56,77]}$ One study has looked at the effect of phase shifts between the two detectors on binning of 4DCT data and it was found that choosing the motion of one detector over the other to determine breathing phase would significantly influence $4 \mathrm{D}$ reconstruction $35 \%$ of the time. ${ }^{[101]}$ While the external markers were not a direct measurement of tumour motion, it was concluded that if two external markers are not well correlated with each other, they cannot both be well correlated with the internal target. If a 4DCT is only used as a measure of tumour excursion one detector may suffice, but if gated therapy is intended then several detectors may be needed to accurately define breathing phase. ${ }^{[101]}$ 


\subsubsection{Dose measurements}

The dose measurements taken by Detectors 2 and 3 are shown in figure 4.15 . Detector 1 also collected dose information; however the location of this detector was far away from the treatment field and doses were negligible $(<5 \mathrm{cGy})$. Detector 2 was inside the treatment field, typically close to the centre, while Detector 3 was at the CT reference isocenter. In several cases, i.e. Patients 5, 7, and 8, Detector 3 was also within or close to the treatment field. The standard deviation of the average dose measured by the RADPOS detector at each point ranged from 2.5 to $11.1 \mathrm{cGy}(2.8$ to $9.2 \%)$ at the site of the tumour and from 3.0 to $13.7 \mathrm{cGy}(7.7$ to $14.0 \%)$ at the $\mathrm{CT}$ reference isocenter. Figure 4.15 displays a comparison between the daily RADPOS dose measurements, $D_{R A D P O S} \pm \sigma_{D_{\text {RDPOS }}}$, where $\sigma_{D_{\text {RDPOS }}}$ comes from the standard deviation of the detector calibration coefficient (approximately $3 \%$ ), and the calculated treatment plan dose, $\hat{D}_{r P} \pm \sigma_{D_{T P}}$ 


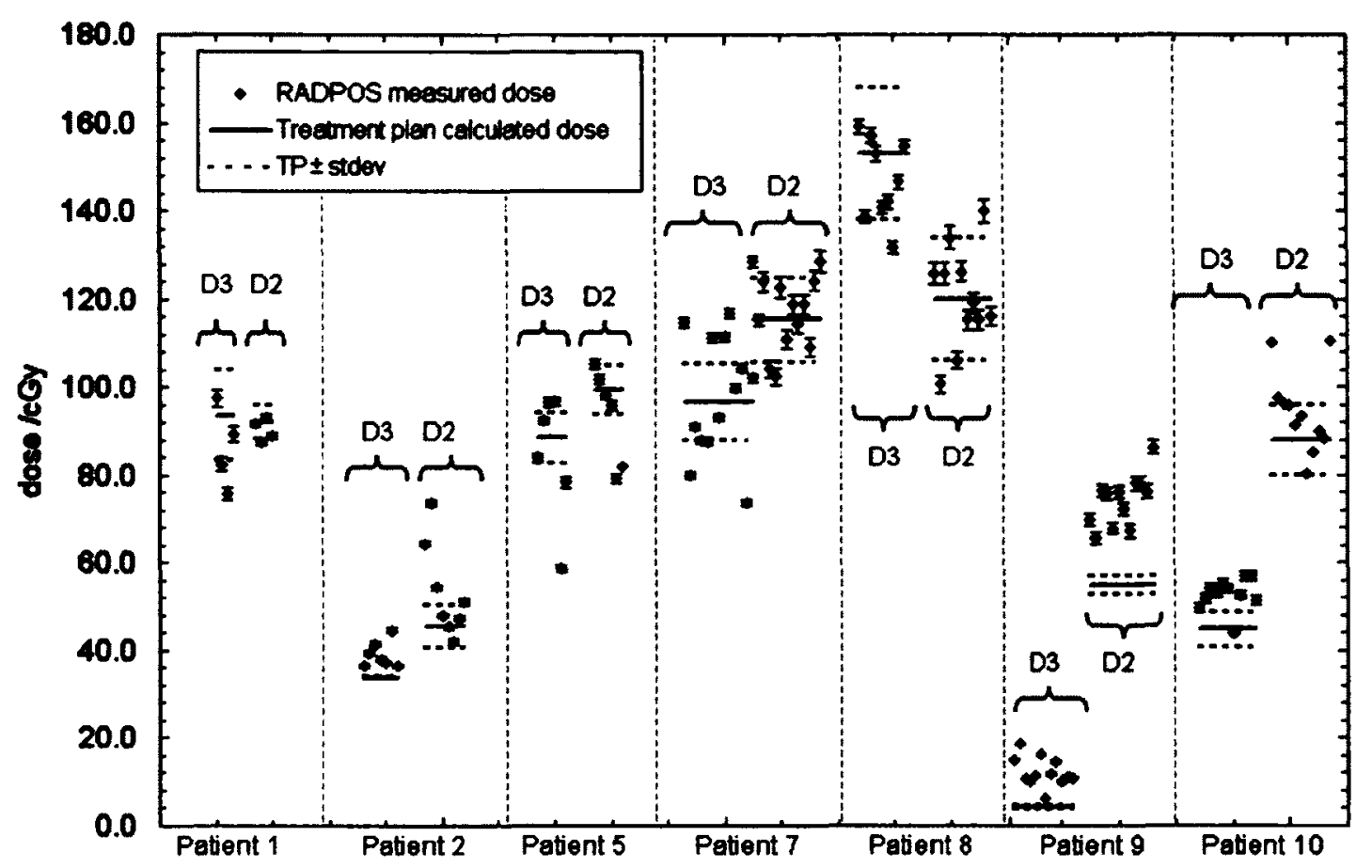

Figure 4.15. Daily RADPOS dose measurements, $D_{R A D P O S}{ }_{ \pm} \sigma_{D_{R A D P O S}}$, compared to the calculated treatment plan dose, $\hat{D}_{T P} \sigma_{D_{T P}}$. For each measurement site, the average treatment plan dose, $\hat{D}_{T P}$, is shown with the horizontal black line, and the upper and lower limits of $\hat{D}_{T P} \sigma_{D_{\pi r}}$ are shown by the blue and red dashed lines respectively.

The average values for each point were in agreement with treatment plan dose for all but three points. For two of the points the delivered dose was less than $50 \mathrm{cGy}$, a threshold below which the uncertainty on standard sensitivity MOSFET measurements increases. This amplified measurement uncertainty along with inaccuracies in treatment plan calculations near the air-surface interface could cause the measurements and dose calculations to be more vulnerable to slight inaccuracies in positioning, limitations in the treatment planning calculations, and machine output variations. 


\subsection{CONCLUSIONS}

The RADPOS system can be used to monitor inter- and intra-fraction variations in breathing patterns and to measure surface dose without any disruption to the treatment schedule or discomfort to patients. Significant deviations in external surface motion have been found throughout the course of treatment that agree with similar studies done elsewhere and which emphasize a need for continued position monitoring. The RADPOS system allows for real-time interpretation of quantitative displacement measurements that are in agreement with clinically-accepted systems, as well as simultaneous measurements of delivered dose. For the study described here, dose was read at the end of each treatment fraction which allowed for comparison with treatment plan values as well as day-to-day consistency. The system does however lend itself to real-time dose measurements, a feature that will be used in future applications. While external surface motion measurements do not provide a direct measure of internal tumour motion, valuable information about the consistency and nature of breathing can be obtained which can be of great clinical value. 


\section{Chapter 5}

\section{REAL-TIME MEASUREMENT OF URETHRAL DOSE AND}

\section{POSITION DURING PERMANENT SEED IMPLANTATION FOR}

\section{PROSTATE BRACHYTHERAPY}

\subsection{INTRODUCTION}

\subsubsection{Prostate cancer}

Cancer can form in the prostate when cells mutate and begin to multiply out of control. The disease can remain unnoticed for long periods of time; however it is often eventually signified by pain, difficulty in urinating, erectile dysfunction, and other symptoms. ${ }^{[106,107]}$ Cancer Statistics Canada reports that prostate cancer is the most common type of malignancy diagnosed in males and ranks third for most deaths, behind only lung and colorectal cancers. ${ }^{[1]}$ With a growing emphasis on prostatic-specific antigen (PSA) based early detection testing, ${ }^{[108]}$ combined with our aging population, the frequency of cases is expected to grow. $[1,109]$

There are several treatment options for patients diagnosed with localized prostate cancer including radical prostatectomy, active surveillance, hormonal therapy, external beam radiation therapy, and brachytherapy. ${ }^{[110]}$ Studies have shown that active surveillance is an appropriate choice for older patients with low volume prostate cancer who have a life expectancy of less than 10 years. ${ }^{[10]}$ Based on the stage of the cancer and the patient's age and overall health, it may instead be decided to pursue surgery or 
radiation therapy to actively treat the disease. ${ }^{[110]}$ Radiation therapy is often prescribed in early stages to stop the spread of disease or in late stages to reduce pain. One method of radiation therapy commonly used to manage prostate cancer is transperineal interstitial permanent prostate brachytherapy (TIPPB). This technique involves treating the tumour with radiation from iodine- 125 or paladium-103 seeds that are permanently placed inside the prostate. This allows for intra-prostatic doses higher than what is achievable with external beam radiation therapy and also has the convenience of a single-day out-patient procedure. ${ }^{[11]}$ The prostate gland is located behind the pubic bone in front of the rectum and directly below the bladder, as shown in figure 5.1. It also surrounds a portion of the urethra, referred to as the "prostatic urethra". Due to the close proximity of these organs at risk, side effects of the procedure can include sexual dysfunction, urinary symptoms, diarrhea and rectal bleeding. ${ }^{[110]}$

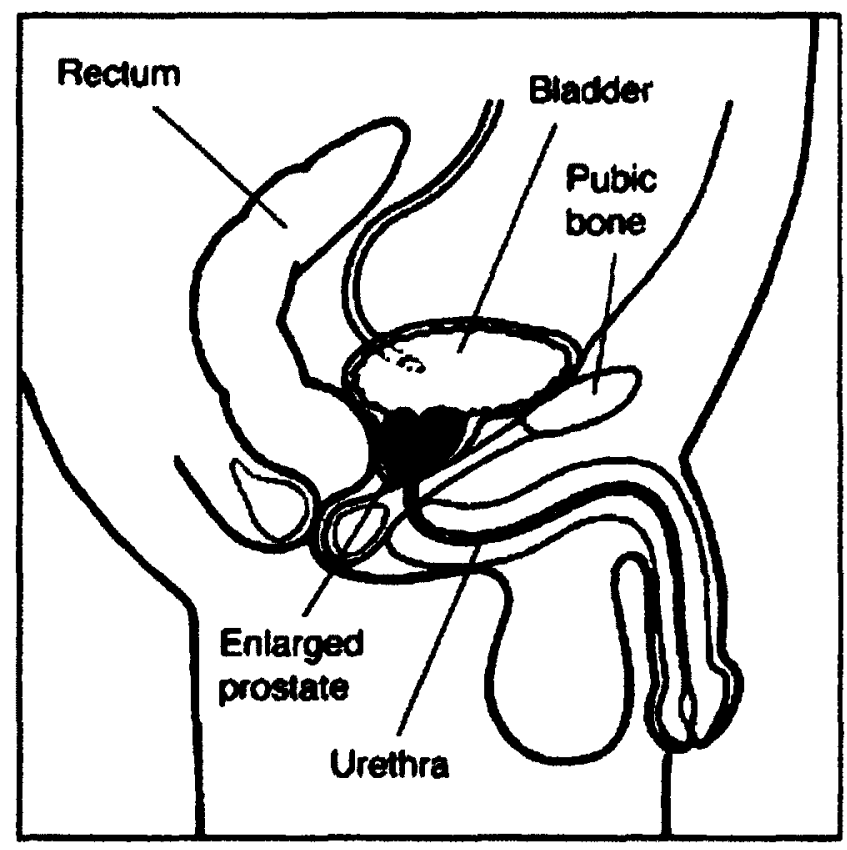

Figure 5.1. Pelvic anatomy. (National Kidney and Urologic Diseases Information Clearinghouse, NIH-063012, Bethesda MD 2006) 
Current practice involves 3D anatomy-based dosimetric planning using ultrasound images taken on the day of seed implantation. At this clinic, images are taken and the treatment plan is created while the patient remains on the treatment couch. This procedure is called 'intra-operative planning' and it improves position reproducibility between the time of imaging and implantation compared to pre-planning, where imaging used for creation of the plan and implantation are done on separate days. Once the treatment plan is complete, needles are loaded with the appropriate number of seeds to achieve the desired configuration. The transperineal insertion of the needles into the prostate is carefully done under the guidance of real-time ultrasound images and a template that is located above the trans-rectal ultrasound (TRUS) probe, see figure 5.2.

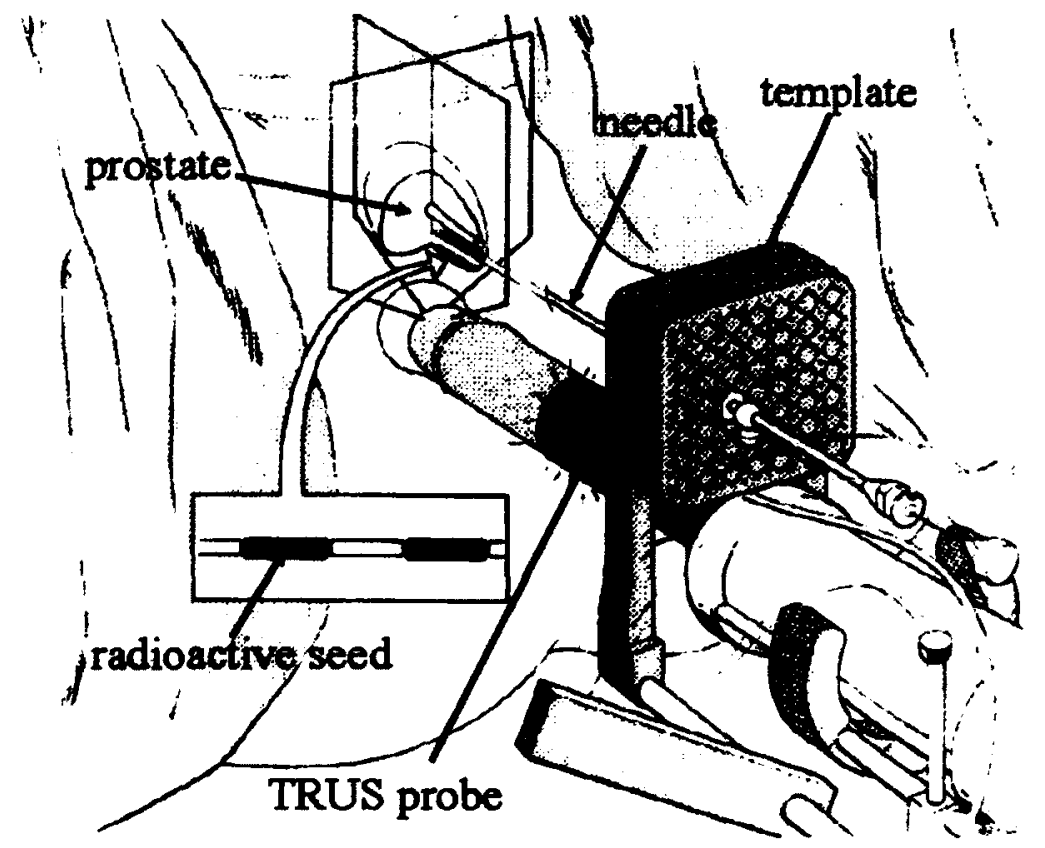

Figure 5.2. Set-up for transperineal interstitial permanent prostate brachytherapy (TIPPB). ${ }^{\text {[12] }}$ 
Once a needle is at the correct longitudinal position, the seeds are inserted into the prostate and the needle is retracted, as shown in figure 5.3. This procedure is repeated until all seeds have been put in place. At that time, the final location of the seeds inside the prostate is assessed and additional seeds may be added based on the estimated dose distribution. Once the procedure is complete, the patient spends a short time in recovery and can leave the hospital the same day. The seeds remain implanted permanently, and after one month a follow-up CT scan is done to assess the position of the seeds at that time and evaluate deviations from the planned dose distribution.
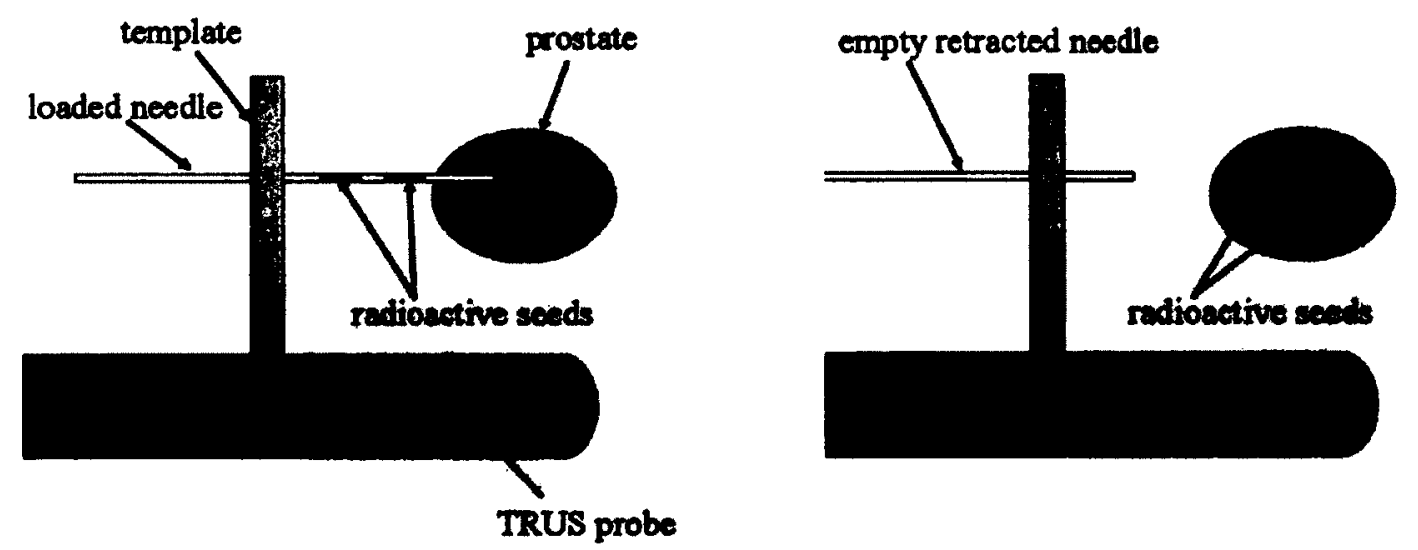

Figure 5.3. Transperineal radioactive seed implantation guided by transrectal ultrasound (TRUS).

\subsubsection{Prostate motion}

Delivered dose to the target volume and nearby organs at risk can deviate from planned values due to uncertainties in needle placement and seed migration. ${ }^{[113]}$ These factors can lead to inadequate dose coverage of the target or compromised sparing of organs at risk. Dose to the urethra, bladder and rectum are of particular concern due to the organs' proximity to the prostate. Swelling and movement of the prostate are also 
significant problems since such changes can affect internal anatomy and dose distribution by making it difficult to place the seeds in the desired pre-planned positions.

Prostate motion during external beam radiation therapy has been widely studied, ${ }^{[114-121]}$ however measured data from TIPPB procedures are much less common. Intra-operative planning involves creating the treatment plan on the actual day of treatment without moving the patient between imaging and needle insertion. This is a recent advancement in TIPPB practices which reduces the concern of inter-fraction or day-to-day motion compared to previous methods where planning and implantation occurred on different days. Intra-fraction motion however, remains a primary concern as studies have shown large magnitudes of both periodic and random motion of the prostate over short time intervals. ${ }^{[122]}$ The causes of such motion can include breathing, cardiac and gastrointestinal functions. ${ }^{[122]}$ Patients are also under the influence of light anesthesia during the procedure which can result in reduced inhibitions and involuntary patient movement.

Prostate size and location can be determined using trans-rectal ultrasound, fluoroscopy, MRI or CT. ${ }^{[123-126]}$ Electromagnetic positioning systems have recently been used to evaluate motion but thus far only during external beam treatments. ${ }^{[127]}$ Several studies have looked at prostatic edema or swelling due to the implantation procedure using various imaging techniques to image the prostate before and after implantation. ${ }^{[123-}$ 126] Sample sizes ranged from 10 to 61 patients and the magnitude of motion was characterized in the left-right, anterior-posterior, and superior-inferior directions separately. It was found that the prostate expands very little in the left-right direction and substantially in the other two directions. Reports on the time course of edema have 
varying conclusions, including indications that the prostate expands exponentially throughout the course of implantation and other reports that the expansion is more linear. The magnitude of expansion measured ranged from 5 to $65 \%$ of total prostate volume. ${ }^{[128-131]}$ Another study has looked specifically at the rotation of the prostate due to needle insertion and found that the prostate had relatively large rotations during implantation in both the saggital and coronal planes. ${ }^{[132]}$ The rotations only correlated with needle insertion in the coronal plane, as rotations in the saggital plane occurred randomly. Motion and deformation of the prostate during implantation have also been quantified using axial ultrasound images and a median change of $1.5 \mathrm{~cm}$ (range of 0.0 to $3.0 \mathrm{~cm}$ ) in the base position of the prostate was reported. ${ }^{[124]}$ The same study also found a median deformation of $6.8 \mathrm{~mm}$ and median displacement of $1.9 \mathrm{~mm}$ in the $x-y$ (transverse) plane. Shifting of the prostate can potentially result in poor seed distribution in the base of the prostate, compromising the dose distribution in this region.

As mentioned above, electromagnetic positioning systems have previously been used to measure prostate motion, however to date all measurements have been taken during external beam radiotherapy. ${ }^{[127]}$ The study described in this chapter involved placing the RADPOS detector inside a urethral catheter during permanent prostate brachytherapy implantation to track displacement in real-time throughout the procedure. The effects of needle insertion, placement of the transrectal ultrasound probe, and patient motion were monitored to evaluate deviations in internal anatomy from time of initial ultrasound imaging. 


\subsubsection{In vivo dosimetry for prostate brachytherapy}

While seeds cannot be removed once they are in place, in vivo dosimetry is useful to identify patients who may be at an increased risk for complications due to higher than expected dose values. ${ }^{[133]}$ Real-time in vivo dosimetry has the added potential of providing feedback during the implant procedure regarding whether the dose rate is approaching threshold values and revisions to placement of subsequent seeds are needed. ${ }^{[134]}$ It can also signal when an underdosage of the target volume might occur, allowing the physician to add more seeds if necessary. Final dose distribution is evaluated using a CT scan performed one month after implantation, when prostate edema is believed to be resolved. ${ }^{[133]}$ A report by the AAPM Task Group 64 suggests that using a half life of 9.3 days for prostatic edema, the prostate should return to $12.5 \%$ of its original size by day 28 post-implant. ${ }^{[109]}$ RADPOS dose measurements have the benefit of signaling higher than expected dose values to the urethra before this scan is performed and therefore alerting the physician to possible treatment complications.

The use of MOSFETs for in vivo dose measurements inside the urethra has been limited. A feasibility study first used a single high sensitivity micro-MOSFET (TN1002RDM) inside a Foley catheter during permanent implant procedures and moved it in increments of $1 \mathrm{~cm}$ along the urethra to characterize the dose rate at various points for five patients. ${ }^{[133]}$ Maximum urethral dose rates for different patients ranged from 10$16 \mathrm{cGy} / \mathrm{hr}$, which corresponded to a total absorbed dose of 205 to $328 \mathrm{~Gy}$. Another group collected similar readings using a high-sensitivity linear MOSFET array with five individual MOSFET dosimeters in 29 patients. ${ }^{[135]}$ Data were collected for one hour while the patients were in post-operative recovery and compared to dose rates calculated from the post-implant CT scan. Measured dose rates ranged from 7.3 to $19.9 \mathrm{cGy} / \mathrm{hr}$, 
depending on the location of each MOSFET. These values correspond to a total absorbed dose of 150-409 Gy. The differences between individual MOSFET dose measurements and calculated treatment plan dose rates ranged from 0 to $25.3 \%$, and the overall correlation between the two sets of values was 0.992 . These studies demonstrated the feasibility of using MOSFET linear arrays for evaluation of initial dose rate to the prostate and urethra after implantation.

A method for monitoring urethral dose during the implant procedure has been developed to evaluate planned seed distribution. ${ }^{[134]}$ A MOSFET array was placed in the urethral catheter as described above and measurements were taken during seed implantation. For the latter half of implantation, the urethral dose rate was calculated and compared to the maximum tolerable dose rate. Based on the measurements and potential impact on prostate coverage, placement of subsequent needles was reconsidered. Measurements taken during the implant procedure, with the trans-rectal ultrasound probe in place, were also compared to those taken post-operatively, with the TRUS probe removed. Good agreement was found indicating that the presence of the ultrasound probe and resulting changes to internal anatomy do not disturb measured dose inside the urethra.

The new in vivo dosimetry tool, RADPOS, combines MOSFET dosimetry with an electromagnetic positioning sensor for simultaneous measurement in real-time of dose and spatial position. ${ }^{[47]}$ The detector has recently been modified to include a MOSFET array rather than a single MOSFET to allow for dose monitoring at five different points along the detector axis. This modified RADPOS detector has been used as part of a clinical trial that was the first to measure both urethral dose and internal motion 
concurrently during permanent seed implants for prostate brachytherapy using a single probe.

\subsection{MATERIALS AND METHODS}

Data were collected for a total of 17 patients. The project was approved by the Ottawa Hospital Research Ethics Board and all patients gave informed consent before participating in the study. The consent form signed by participants can be found in Appendix B. The RADPOS probe, shown in figure 5.4, contains five high sensitivity MOSFETs (Best Medical, Ottawa, ON, Canada) and a high bias supply setting was used for all measurements. The RADPOS probe was secured inside a Foley catheter which was then inserted into the patient's urethra. The detector is waterproof; however plastic wrap was secured around the part of the wire not inside the catheter for additional protection. Sterilization procedures were conducted before and after each use. The magnetic field transmitter was then positioned on the treatment couch within the recommended range of the detectors. ${ }^{[47]}$

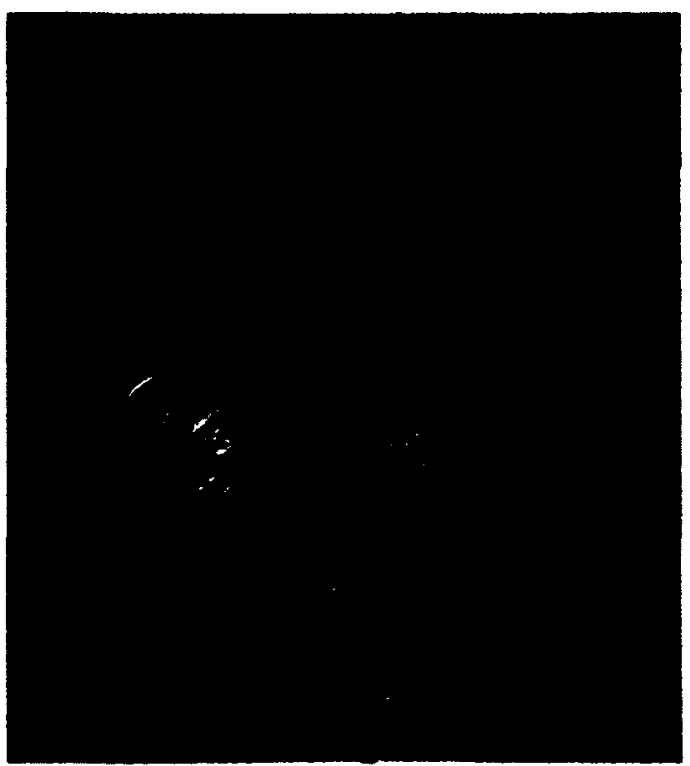

Figure 5.4. RADPOS detector with MOSFET array. 
Before implantation began, a fluoroscopy image was taken to verify the detector's position. Fluoroscopy images were also taken both before and after TRUS probe removal with the RADPOS probe and all seeds in place. The positions of the MOSFET dosimeters could be inferred using the marker located at the end of the RADPOS wire which is visible on the images, as shown in figure 5.5. The MOSFETs are located $2 \mathrm{~cm}$ apart, beginning $0.8 \mathrm{~cm}$ away from the marker, and the position sensor is located between the third and fourth MOSFETs. These five dose points will be referred to numerically starting with MOSFET 1 which is closest to the marker and therefore closest to the bladder. MOSFET 5 would be furthest from the bladder. Although probe position varied slightly between patients, this set-up generally resulted in one dose point inside the bladder (MOSFET 1), two or three dose points within the prostatic urethra (MOSFETs 24), and one or two dose points at or inferior to the apex of the prostate (MOSFETs 4-5). Figure 5.6 displays the typical position of the RADPOS array with respect to pelvic anatomy. 


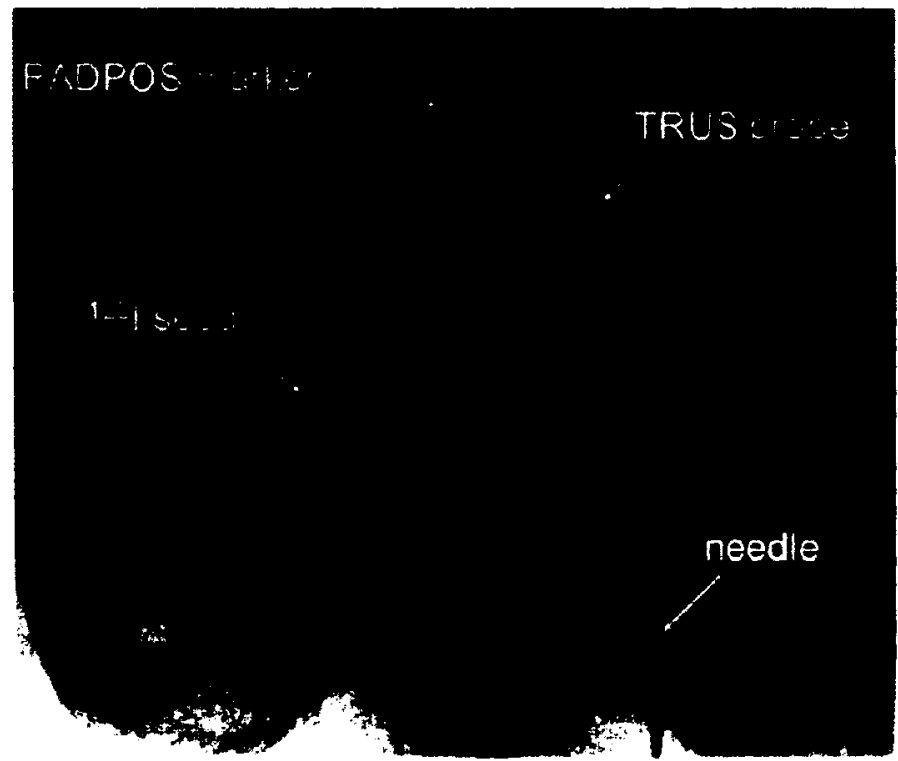

Figure 5.5. Fluoroscopy image taken during implant procedure. RADPOS marker can be seen inside the Foley catheter balloon.

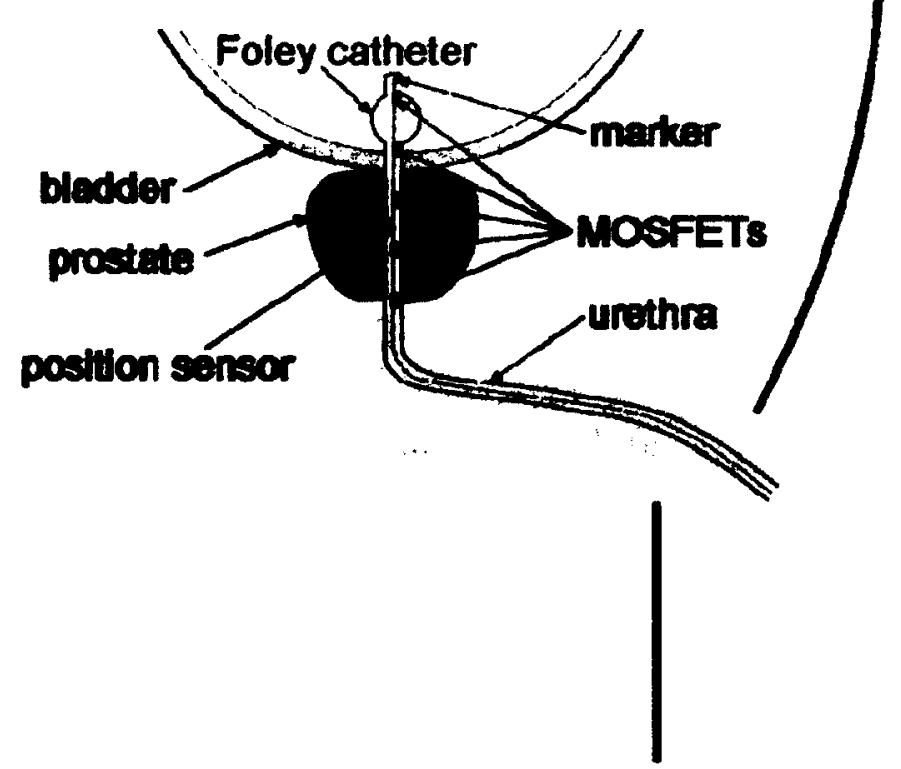

Figure 5.6. Typical position of RADPOS array with respect to pelvic anatomy during TIPPB procedure. 
Spatial coordinates of the RADPOS detector were read every $0.5 \mathrm{~s}$ throughout the duration of the procedure (1-1.5 hr) including treatment planning and implantation. The timing of events such as needle insertion and movement of the trans-rectal ultrasound (TRUS) probe was noted. An initial MOSFET reading was taken once all seeds had been implanted and then again ten minutes later. The TRUS probe was then removed and another ten minute reading of the MOSFETs was completed. The change in threshold voltage of each MOSFET was multiplied by a MOSFET-specific calibration coefficient to get the dose over ten minutes in centigray, $D(10 \mathrm{~min})$. Due to the geometry of the five MOSFETs and radiation protection concerns, the MOSFETs were calibrated using a 100 $\mathrm{kVp}$ orthovoltage beam rather than actual iodine-125 seeds. The mean beam energy was $30 \mathrm{keV}$, which is the approximate energy of the $\mathrm{x}$-rays emitted from the seeds. Equation 5.1a and b were then used to calculate dose rate in centigray per hour, $\dot{D}_{0}$, and total integral dose in gray, $D$. In equation $5.1 \mathrm{~b}, T_{1 / 2}$ represents the half-life of the seeds, which for the iodine-125 used in these procedures is 59.4 days or 1425.6 hours.
a) $\dot{D}_{0}=D(10 \mathrm{~min}) \times 6^{c G y} / \mathrm{hr}$
b) $D=\left(\dot{D}_{0} \times 1.44 \times T_{1 / 2}\right) / 100 G y$

\section{Equation 5.1}




\subsection{RESULTS \& DISCUSSION}

\subsubsection{Dose measurements}

The maximum total integral dose for each patient in the prostatic urethra ranged from $D_{\max }=89-195 \mathrm{~Gy}$ for all patients followed. An accepted maximum dose for this organ is $200 \mathrm{~Gy},{ }^{[134]}$ so the measurements did not indicate any potential overdose. Irritation of the urethra is the main cause of symptoms following prostate brachytherapy, so it is valuable to confirm that delivered dose does not exceed this limit. ${ }^{[133]}$ The expected dose profile along the urethra was determined from treatment planning calculations. Based on the position of the marker (which is visible on the images) the locations of the MOSFETs were deduced and dose measurements were compared to the appropriate treatment plan values. An example of this comparison is shown in figure 5.7, with distance from the RADPOS marker plotted along the $x$ axis. For this patient, the maximum dose measured by the RADPOS was within $7.3 \%$ of the treatment plan value at the same point. MOSFETs 1 and 3 (the left and middle dose points) also show good agreement in the lower dose regions.

The initial procedure involved dose measurements only after the TRUS probe had been removed, however comparisons showed measurements were systematically lower than the calculated treatment plan values. Figure 5.8 shows the differences between the total integral dose calculated from RADPOS measurements and from treatment plan calculations for these initial patients. To account for the small dose values measured by the dosimeters located beyond the limits of the prostatic urethra, the differences for all measurement points were normalized to the maximum treatment plan value for each specific patient. For example, the maximum treatment plan value among the five dose 
points for Patient 1 was at the location of MOSFET 2, $D_{T P 2}$. The plotted values for Patient 1 are therefore as follows:

$$
\begin{aligned}
& \Delta_{1}=\left[\left(D_{T P 1}-D_{R A D P O S}\right) / D_{T P 2}\right] \times 100 \\
& \Delta_{2}=\left[\left(D_{T P 2}-D_{R A D P O S 2}\right) / D_{T P 2}\right] \times 100 \\
& \Delta_{3}=\left[\left(D_{T P 3}-D_{R A D P O S 3}\right) / D_{T P 2}\right] \times 100 \\
& \Delta_{4}=\left[\left(D_{T P 4}-D_{R A D P O S 4}\right) / D_{T P 2}\right] \times 100
\end{aligned}
$$

\section{Equation 5.2}

Treatment plan values were not available for the location of MOSFET 5 (most inferior to the bladder) since it was consistently located outside of the prostatic part of the urethra, therefore data are only shown for MOSFETs 1-4. Another study comparing MOSFET array urethral dose measurements with treatment plan calculations for TIPPB found that the correlation between the two values decreased with increasing dose. ${ }^{[135]}$ In that work, the agreement between the MOSFETs and treatment plan ranged from 0 to $25.3 \%$ and it was proposed that dose measurements could help reconcile the lack of correlation between urethral complications and calculated dose that has been noted by several investigators. ${ }^{[135]}$ Based on the measurements presented in this chapter, as well as previously reported variations between measured and calculated dose, ${ }^{[133-136]}$ it was suspected that anatomical changes (especially prostate shape deformation) due to removal of the probe could have caused the MOSFETs to shift in position with respect to the seeds. For this reason, measurements were taken both before and after the removal of the probe for the most recent patients. 


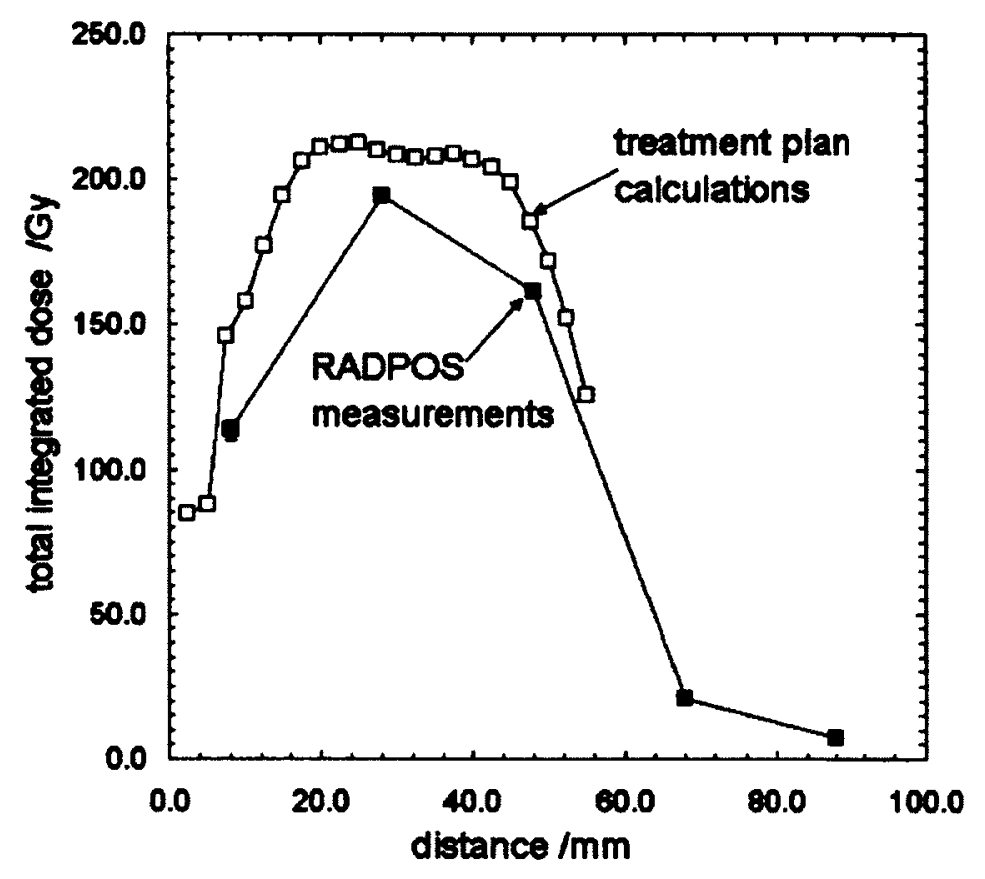

Figure 5.7. RADPOS dose measurements and calculated treatment plan dose values for Patient 6 .

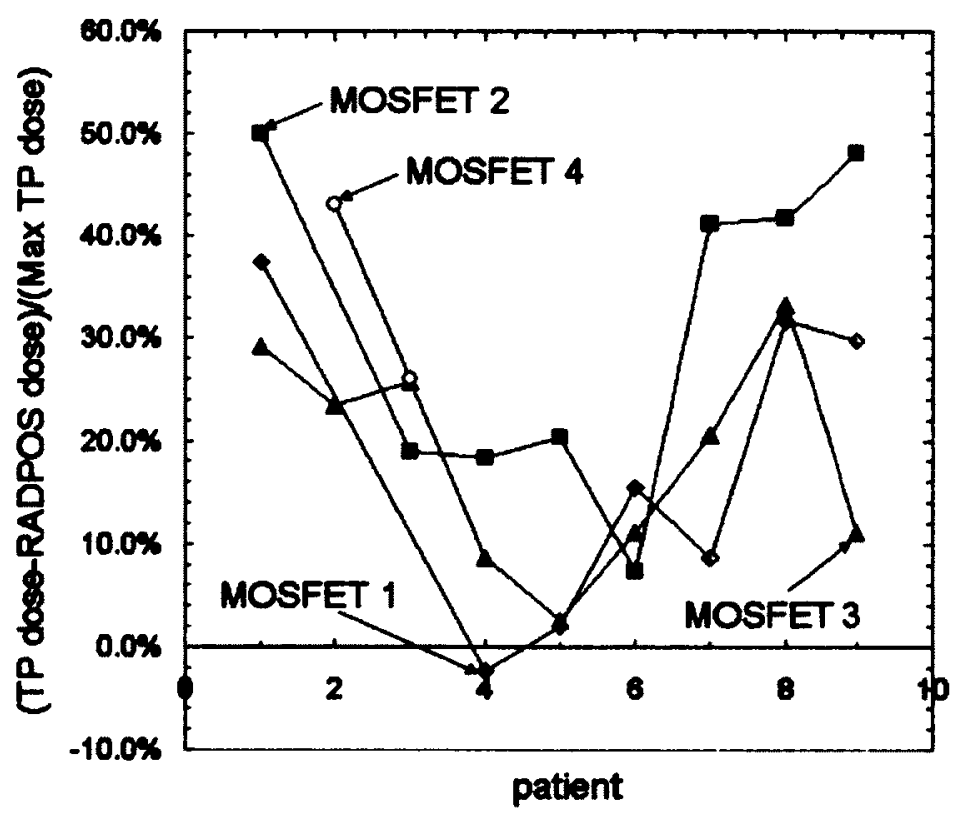

Figure 5.8. Comparison of total integral dose calculated from RADPOS dose measurements and treatment plan values. For each patient, the differences for all MOSFET locations were normalized to the maximum dose measured for that patient. 
Measurements were taken both before and after the removal of the TRUS probe for a total of ten patients. Differences between the two sets of measurements varied between patients, and examples of the different types of results found are shown in figure 5.9. For each plot, the calculated total integral dose for each point is normalized to the maximum value with the probe in. The only case where the dose increased when the probe was removed was Patient 13, shown in figure 5.9b. The rest of the patients showed either a decrease in dose (figure 5.9a) or no difference (figure 5.9c) once the probe was removed therefore agreement with treatment plan values was not improved by taking the second set of measurements. It did however, highlight the possible differences in dose distribution when the probe is removed, an effect that warranted further investigation.

a)

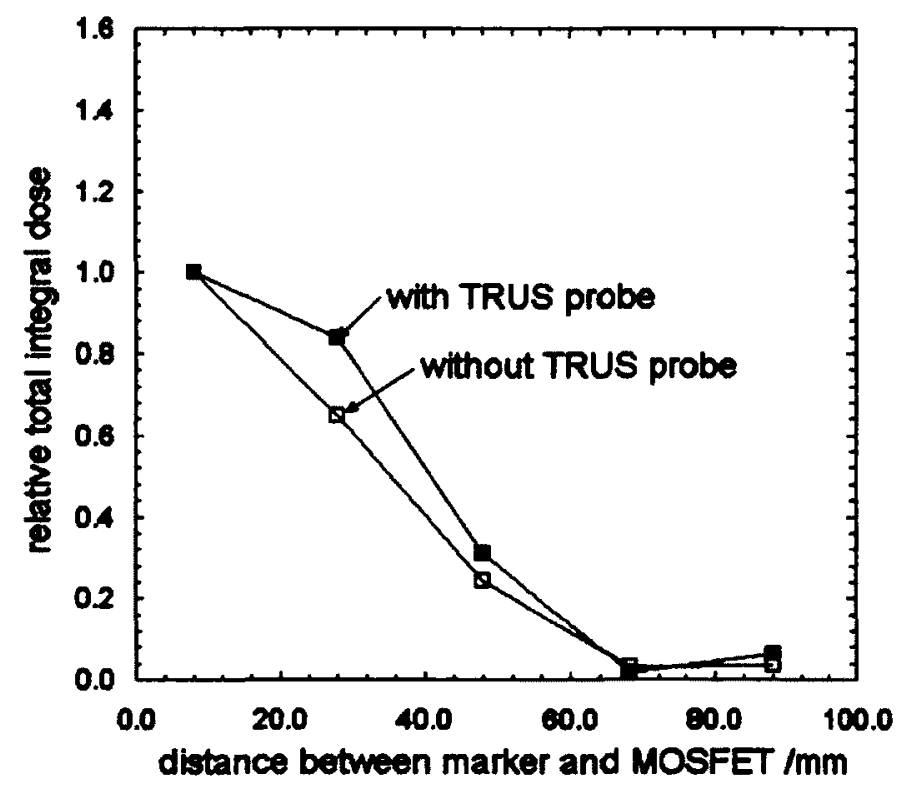


b)

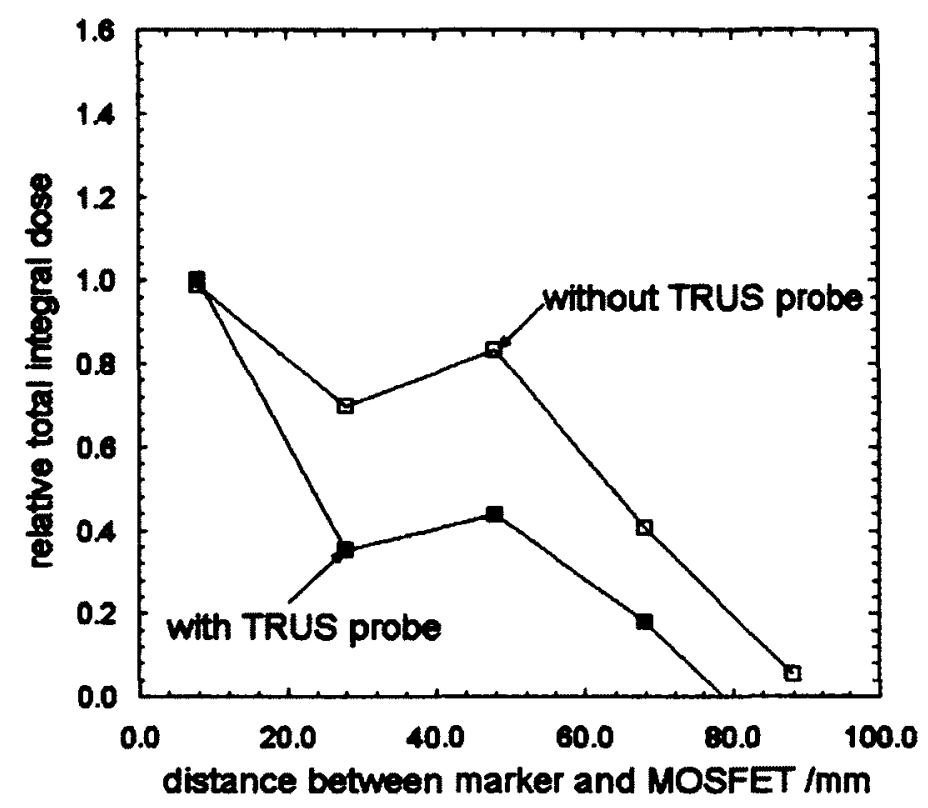

c)

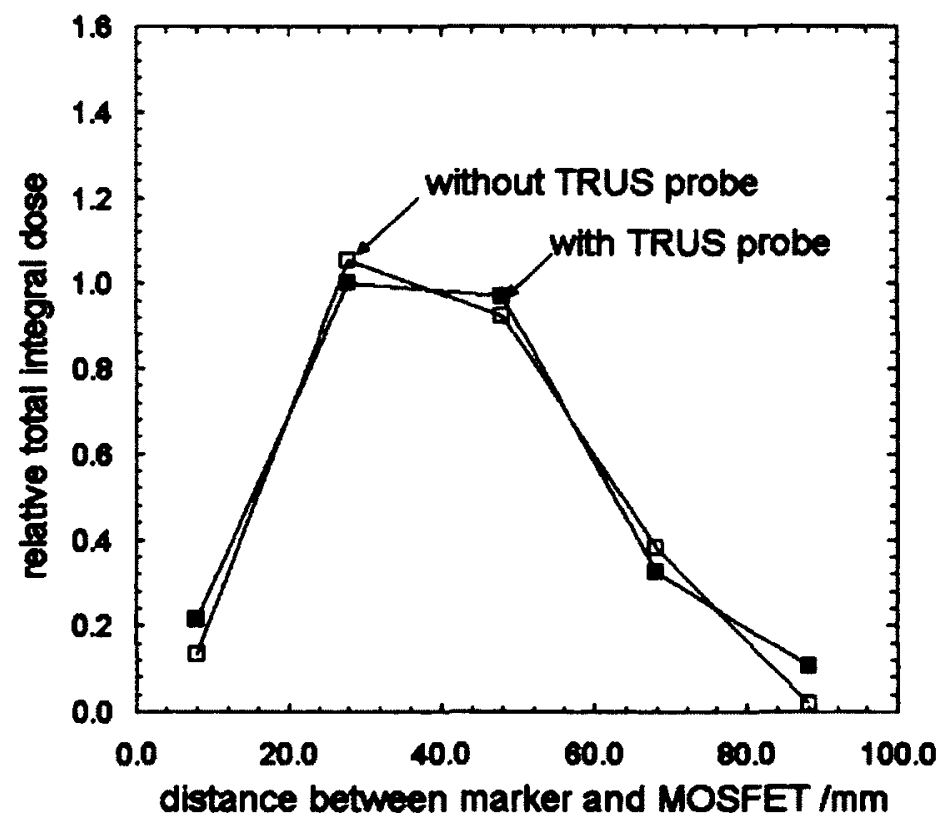

Figure 5.9. Comparisons of relative dose measurements taken before and after the TRUS probe was removed for a) Patient 13, b) Patient 7, and c) Patient 16 . In each case, the total integral dose values have been normalized to the maximum integral dose for all MOSFETs with the TRUS probe in place specific for the patient presented. Errors bars are not visible because they are smaller than the symbols shown. 
Table 5.1 displays the percent difference between the total integral dose values measured with the probe in place, $D_{\text {probe, }}$ and without the probe in place, $D_{\text {no probe, for those }}$ MOSFETs determined to be within the prostate. Variations ranged from $-66 \%$ to $36 \%$, and differences of greater than $20 \%$ were seen for four patients, indicating significant shifts in seed positions relative to the urethra once the probe was removed.

Table 5.1. Percent difference between the total integral dose values measured with the probe in place, $D_{\text {probe, }}$ and without the probe in place, $D_{\text {mo probe, }}$ for those MOSFETs determined to be within the prostate.

\begin{tabular}{|c|c|c|}
\hline Patient & MOSFET & Relative change in dose /\% \\
\hline \multirow{2}{*}{7} & 1 & 1.5 \\
& 2 & -65.8 \\
& 3 & -62.0 \\
\hline \multirow{3}{*}{8} & 1 & 6.0 \\
& 2 & 3.6 \\
& 3 & -5.4 \\
\hline 9 & 1 & -1.7 \\
& 2 & 2.9 \\
\hline 10 & 1 & -4.4 \\
\hline 12 & 1 & -7.7 \\
& 2 & 12.3 \\
\hline \multirow{2}{*}{13} & 1 & -0.1 \\
& 2 & 25.5 \\
& 3 & 23.6 \\
\hline \multirow{2}{*}{14} & 2 & 1.6 \\
& 3 & -13.6 \\
\hline \multirow{2}{*}{15} & 4 & -30.5 \\
\hline 16 & 2 & -2.6 \\
& 3 & -15.5 \\
\hline \multirow{2}{*}{17} & 2 & -5.3 \\
& 2 & 4.8 \\
\hline & 3 & -7.8 \\
& Average: & -2.8 \\
\hline & & -4.5 \\
\hline
\end{tabular}


Another study has compared urethral dose rates measured during the implantation procedure with those measured in the recovery room, once the TRUS probe was removed. ${ }^{[134]}$ It was stated that the two sets of measurements agreed well and differences were within the standard deviation of the values. The conclusion was therefore that the presence of the TRUS probe did not affect dose distribution. In that study, only average values for each MOSFET were compared rather than individual patient measurements. For example, rather than comparing the dose measured by MOSFET 1 for Patient $\mathrm{X}$ during treatment with the dose measured by MOSFET 1 for Patient $\mathrm{X}$ in the recovery room, all measurements made by MOSFET 1 in the treatment room for all patients were averaged and then that average value was compared to the average of all the measurements made by the same MOSFET in the recovery room. This method of analysis is vulnerable to missing individual cases of large differences. To our knowledge no other dose comparisons with and without the TRUS probe have been published.

\subsubsection{Position measurements}

Once the TRUS probe was in place and initial fluoroscopy images were taken, patients remained still while the treatment plan was created. Prostate motion during this period gives a good indication of behaviour without movement of the ultrasound probe or swelling due to needles. Once the treatment plan was complete, the implantation procedure began, which lasted on average $30 \mathrm{~min}$ (range of 12 to $48 \mathrm{~min}$ ) from the time the first needle was inserted until the last. Throughout implantation, short-term displacements due to needle insertion were noted as well as overall shifts, possibly due to prostate swelling. An example of prostate movement due to needle insertion is shown for Patient 6 in figure 5.10. The grey circles represent the time the physician began inserting 
a needle. For the case shown in figure 5.10a, the average displacement during insertion was within $r=1.1 \mathrm{~mm}$. Figure $5.10 \mathrm{~b}$ is a close up of the first few needle insertions to more clearly show the effect of individual needles.

Placement of each needle was done under ultrasound guidance, and in some cases the needle had to be repositioned more than once, causing increased displacement. This was the case for the two instances of large motion in figure $5.10 \mathrm{a}$, which are circled in red. Each needle was recorded during implantation, and the two needles corresponding to the red circled areas were $c 3.5$ and e1.5. The position of these needles within the template is also shown. In these two cases, one of the needles was placed deep into the middle of the prostate, and the other was near the patient's left posterior side and not as deep. The placement of individual needles was analyzed for each patient, and it was found that the magnitude of displacement correlated to occasions when the physician had to reposition the needle multiple times and not with the actual position of the needle with respect to the patient's prostate. 
a)

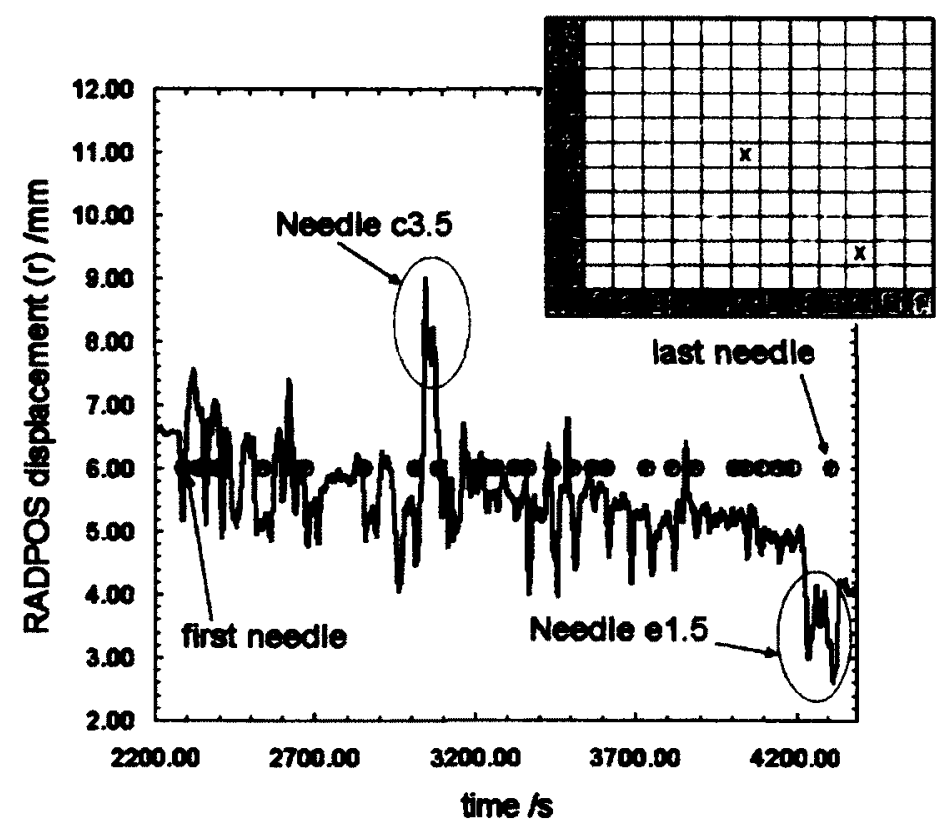

b)

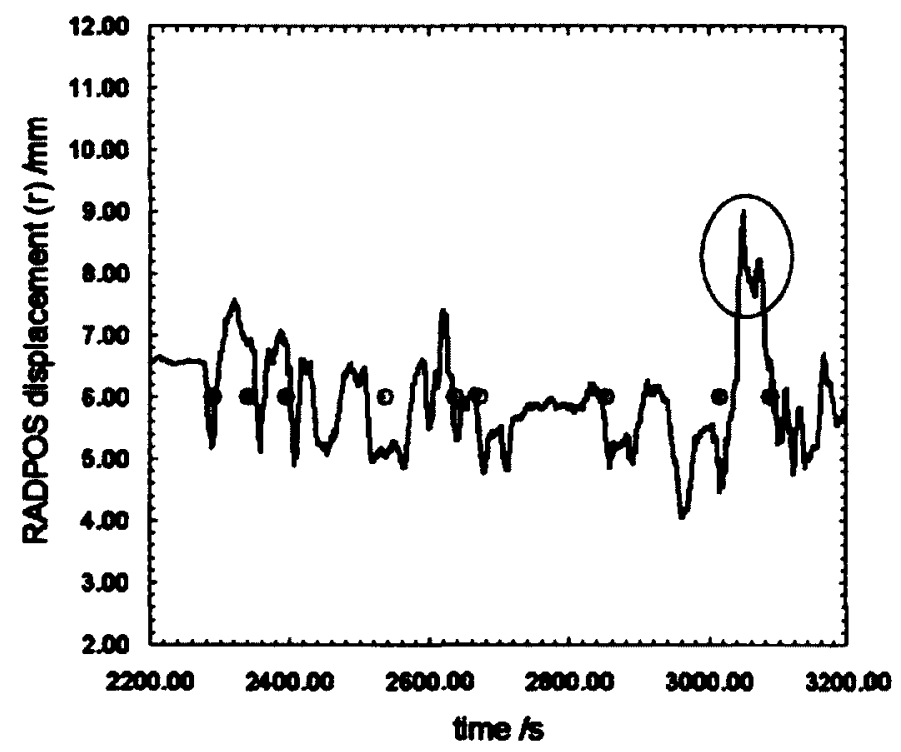

Figure 5.10. Position information collected during seed implantation for Patient 6. The root mean squared position $\left(r=\sqrt{\left(x^{2}+y^{2}+z^{2}\right)}\right)$ is shown by the black line and the grey circles represent needle insertion. 
The range of average displacement in each direction during implantation is shown for all patients in table 5.2. Also presented in this table is the range of displacements measured during treatment planning, as a baseline reference for expected motion. Earlier studies of prostate edema done by other groups have found that the prostate expands very little in the left-right direction and substantially in the other two directions throughout implantation. ${ }^{[123-126]}$ RADPOS position measurements provide data on displacement and not overall volume changes, however swelling of the prostate could shift the prostatic urethra, and therefore the RADPOS positioning detector, in various directions. The range of displacement in the superior/inferior and anterior/posterior directions found in the work presented here were both larger than the magnitude of displacement in the left-right direction, as can be seen in the table below.

Table 5.2. Range of average change in position among all patients during treatment planning, during implantation, and finally due to removal of the TRUS probe.

\begin{tabular}{|l|l|c|c|}
\hline \multirow{2}{*}{ Direction } & \multicolumn{3}{|c|}{ Average displacement } \\
\cline { 2 - 4 } & $\begin{array}{c}\text { during treatment } \\
\text { planning }\end{array}$ & $\begin{array}{c}\text { during implant } \\
\text { procedure }\end{array}$ & $\begin{array}{c}\text { due to TRUS } \\
\text { probe removal }\end{array}$ \\
\hline$x$ (right/left) & $(0.0-0.0) \mathrm{mm}$ & $(0.0-1.8)$ & $(0.1-2.6) \mathrm{mm}$ \\
\hline$y$ (superior/inferior) & $(0.0-0.0) \mathrm{mm}$ & $(0.4-3.9)$ & $(0.3-8.7) \mathrm{mm}$ \\
\hline$z$ (anterior/posterior) & $(0.0-0.0) \mathrm{mm}$ & $(0.0-3.3)$ & $(1.2-5.0) \mathrm{mm}$ \\
\hline$r=\sqrt{x^{2}+y^{2}+z^{2}}$ & $(0.3-1.6) \mathrm{mm}$ & $(1.4-5.1)$ & $(1.4-9.7) \mathrm{mm}$ \\
\hline
\end{tabular}

Since the RADPOS system provides real-time quantitative position information one can see the effect of patient motion or other events on the position of the detector, which is secured in the prostatic urethra. An example of position information collected during the dose measurement periods is shown in figure 5.11 for Patient 8 . In this case, the change in position due to removal of the rectal probe (at approximately $t=4100 \mathrm{~s}$ ) can 
be seen in all coordinates with the largest magnitude along the $y$ (superior/inferior) direction. As mentioned above, trans-rectal ultrasound imaging is used throughout the imaging, planning and implantation procedures. This involves the placement of a probe into the rectum which remains in place until seed implantation is complete. No published research could be found on the effect of this probe on internal anatomy or prostate position. The average displacement due to the removal of the probe is shown in the last column of table 5.2 .

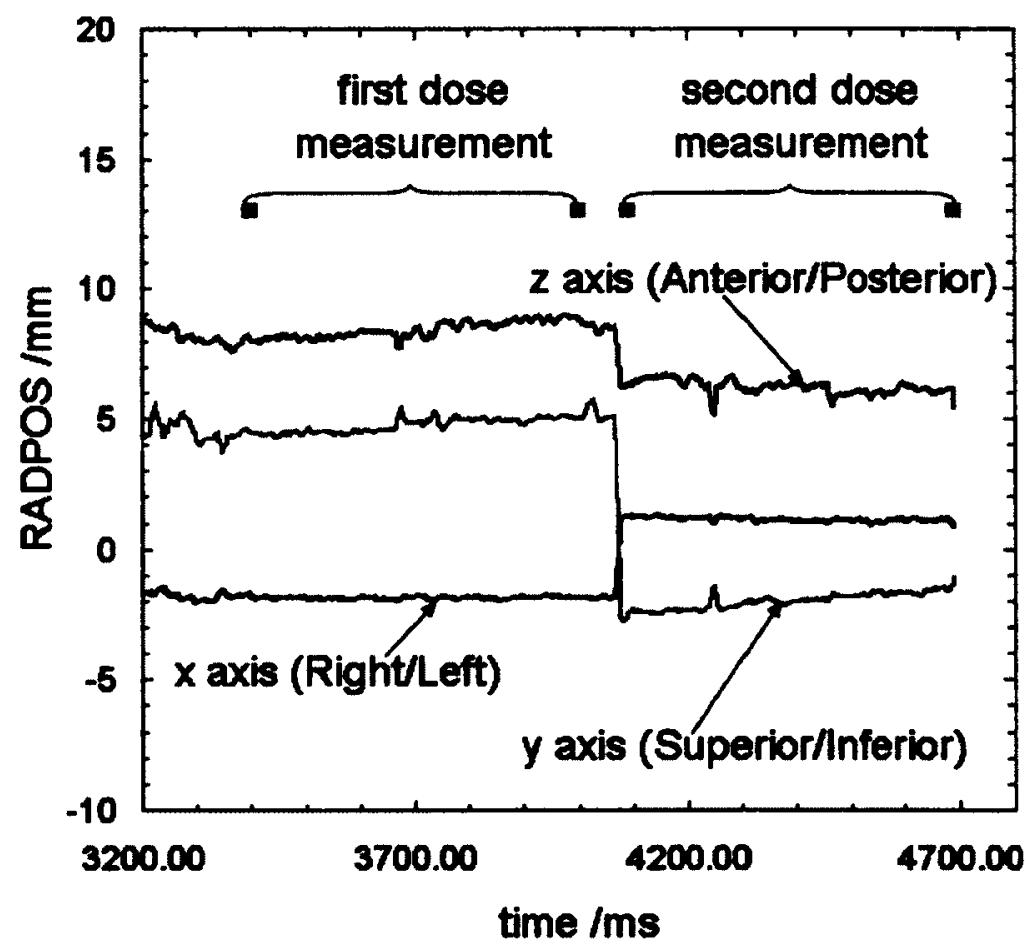

Figure 5.11. RADPOS position measurements for Patient 8 taken after implantation was complete. Black squares represent the time of the initial and final MOSFET readings taken for the first measurement period (with TRUS probe in place) and the second measurement period (after the TRUS probe was removed). The change in position from the removal of the probe can be seen in all coordinates at approximately $t=4100 \mathrm{~s}$. 
To examine whether the changes in dose (shown in table 5.1) with and without the TRUS probe in place correspond with the magnitude of shift in detector position, these data were compared for all relevant patients. A significant correlation was not found between the two sets of values, however individual anatomy varies greatly so movement may need to be analyzed on a case by case basis rather than as a whole. Instead of considering total magnitude of displacement of the probe relative to the transmitter on the treatment couch, it may be more telling to analyze how positions of seeds change relative to the RADPOS detector as the prostate changes shape with removal of the ultrasound probe.

To accurately investigate these changes, 3D CT information taken before and after TRUS probe removal would be needed. This is not part of routine practice at this hospital as CT data are collected only one month after the implantation. A detailed analysis was therefore not possible. Two dimensional fluoroscopy images are taken however, allowing for a crude estimation of these relative changes. Three examples are shown here in figure 5.12 for Patients 8,10 , and 16. The images show the post-implant fluoroscopy image once the TRUS probe has been removed. Superimposed on top of the images are the outlines of the seed and probe positions from the fluoroscopy images taken while the probe was still in place. Seed outlines are shown as red lines while the RADPOS marker (when visible) is shown in yellow. Boney anatomy and TRUS probe outlines are shown in black and all geometry has been registered to the pubic arch. Seed outlines that are missing were blocked in the image by the TRUS probe. It can be seen from these examples, that seed positions have a tendency to shift away from the base of the prostate, in some cases by significant amounts, when the probe is removed. Three- 
dimensional analysis would allow for quantification of these shifts and their potential affect on dose distribution. However, imaging needed for such analysis is not available in our department.

a)

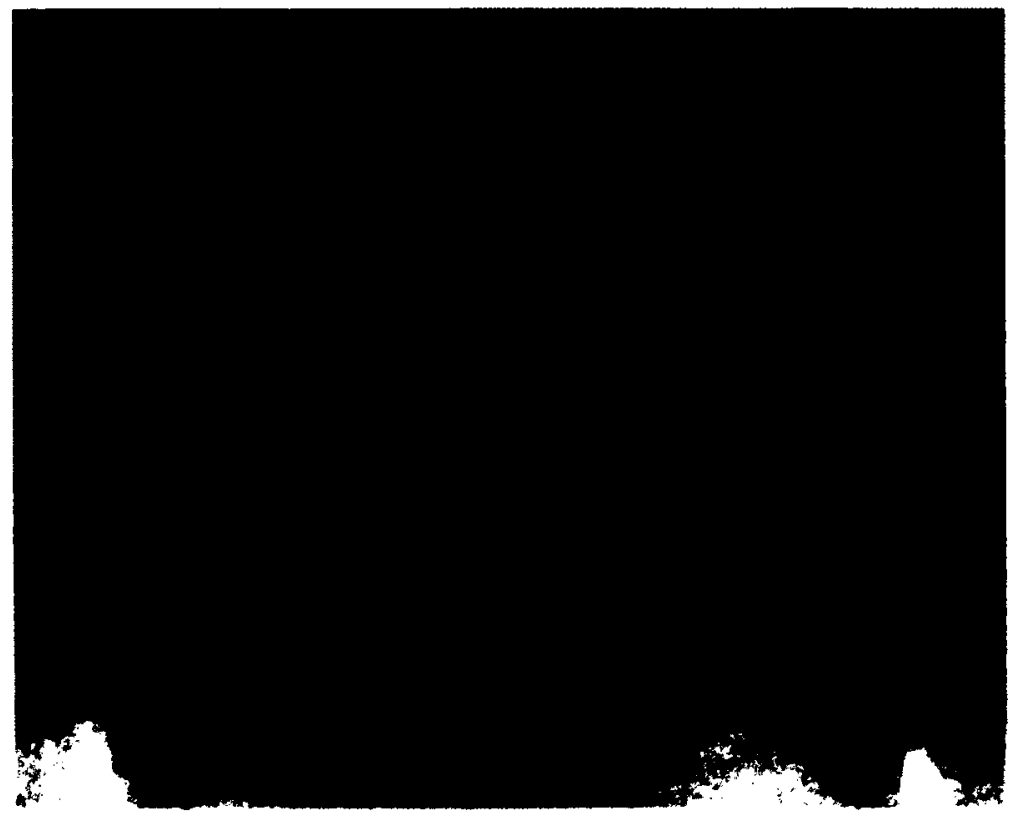

b)

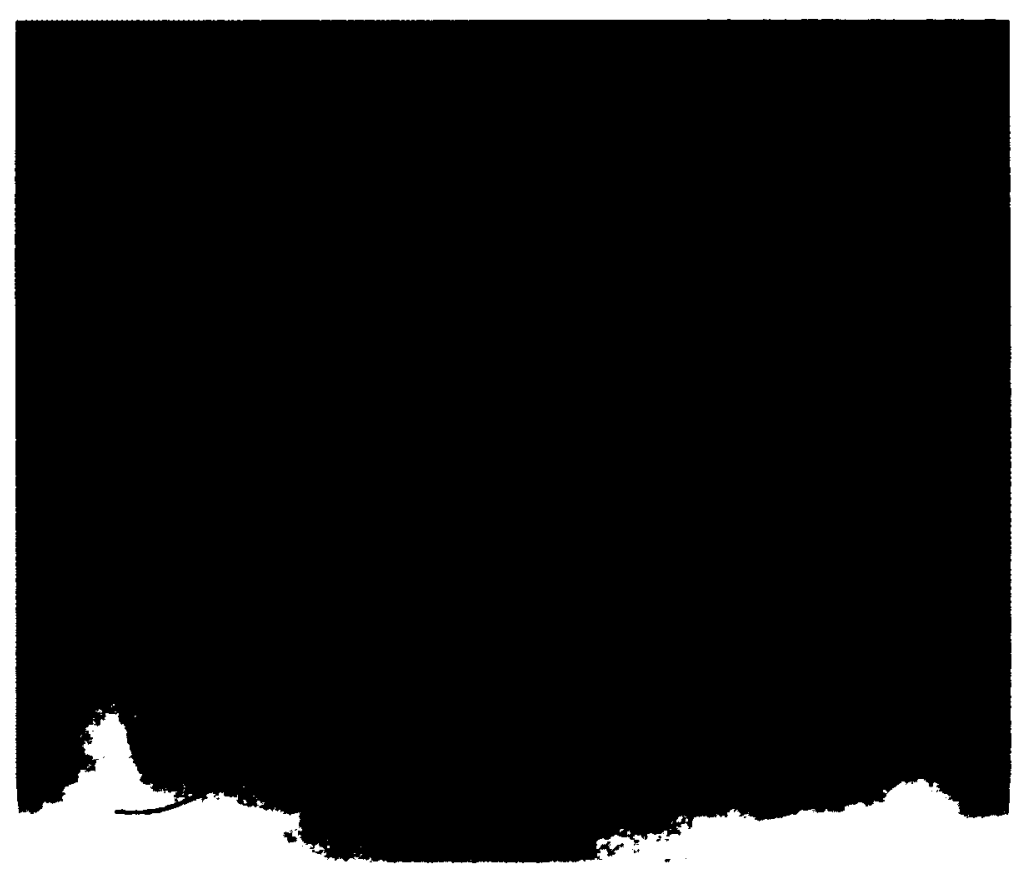


c)

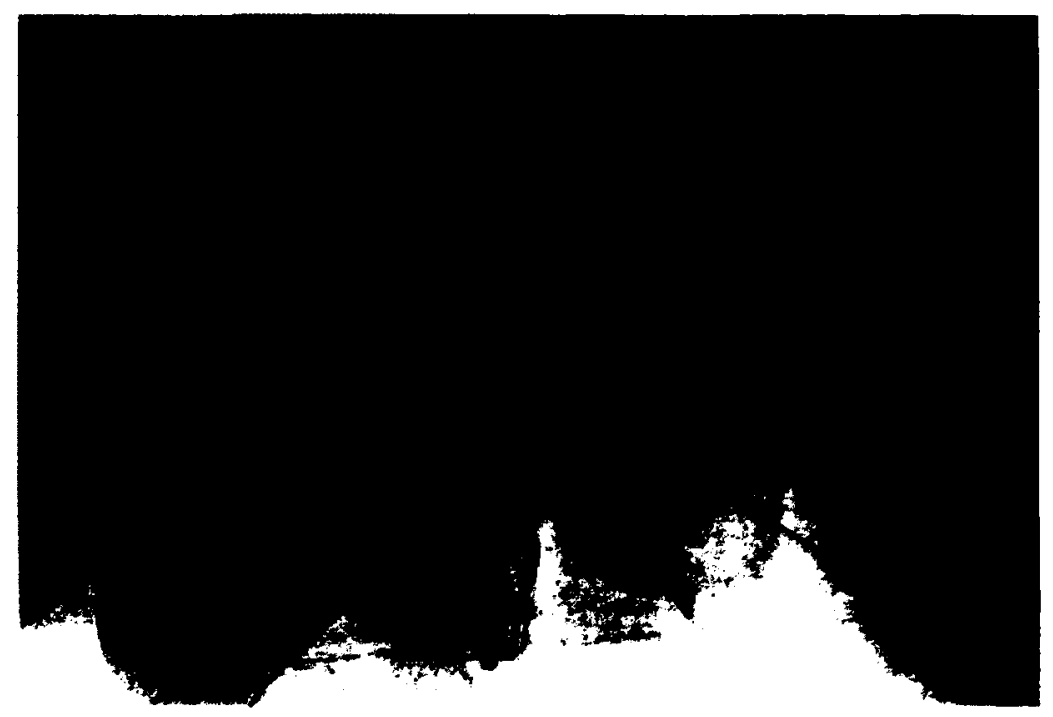

Figure 5.12. Fluoroscopy images taken post-implant once the TRUS probe was removed for Patients a) 8 b) 10 and c) 16. Outlines of seed, probe, and RADPOS marker positions from before the probe was removed are shown to display displacements. Outlines have been registered to the pubic arch.

\subsection{CONCLUSIONS}

The modified RADPOS detector with MOSFET array is able to provide real-time dose information which can be used to monitor dose rates while implantation is performed and to estimate total integrated dose. This information can potentially be used to guide subsequent needle placement as well as to identify patients who may be at an increased risk for complications. Deviations from calculated treatment plan values have been noted, therefore in vivo dosimetry should be used to verify actual delivered dose. Changes in position due to needle placement, swelling, or other internal motion as well as changes due to the TRUS probe can be significant and should be quantified to evaluate influence on dose distributions. 


\section{Chapter 6}

\section{CONCLUSIONS}

This thesis covers experiments done to bring a prototype of the RADPOS system from initial testing to implementation into clinical routines. Chapter 2 reviewed investigations of the dosimetric and position-measuring capabilities of the system in a lab setting before any patient measurements were done. Results indicated that RADPOS dose measurements were within $5 \%$ of diode measurements for ${ }^{60} \mathrm{Co}$ and within $3 \%$ for commonly used high-energy beams and the angular response was on average within $1.6 \%$ over $360^{\circ}$. This deviation in angular response is slightly higher than when microMOSFETs are used alone, perhaps due to increased scatter from the position monitor. Specific calibration coefficients should be determined for each detector, and RADPOS probes with a high sensitivity MOSFET should be used when the highest level of precision is required. These MOSFETs will however become saturated and therefore unusable at lower doses compared to standard sensitivity MOSFETs. It was found that the position readout was stable within $0.2 \mathrm{~mm}$ over a period of an hour and displacement measurements can be obtained with the highest accuracy when interfering metals are at least $200 \mathrm{~mm}$ away. RADPOS detectors should remain within an optimal operating range with respect to the magnetic field transmitter to ensure a consistent accuracy of $\pm 2 \mathrm{~mm}$ on 3D displacements. This range is defined by the following coordinates: $150-470 \mathrm{~mm}$ along the $x$-axis, $\pm 215 \mathrm{~mm}$ along the $y$-axis, and $\pm 100 \mathrm{~mm}$ along the $z$-axis. 
A rigid dynamic phantom was first used to simulate patient motion due to breathing and preliminary tests showed the potential of the RADPOS system to accurately measure displacements similar to chest wall movement. To explore further possible clinical applications, the system was then used in conjunction with a deformable lung phantom to simulate both free breathing and adaptive radiation treatment deliveries, as described in Chapter 3. The RADPOS system was used to verify delivered dose and motion of a simulated tumour while imitating a real patient treatment. The accuracy of RADPOS position measurements was within $2.5 \mathrm{~mm}$ and relative displacement measurements were preserved within $0.7 \mathrm{~mm}$ between $\mathrm{CT}$ simulation and radiation delivery rooms. Dose measurements were within $5 \%$ of treatment planning calculations for the free-breathing delivery and agreement was within $1.5 \%$ for the adaptive delivery, which was well within the estimated experimental uncertainties. This work demonstrated that the deformable lung phantom-RADPOS system could be used as an efficient quality assurance tool for $4 \mathrm{D}$ radiation therapy acting as an end-to-end treatment verification instrument.

The RADPOS system was then used in two different clinical trials, reviewed in Chapters 4 and 5. The first trial involved measurements on patients undergoing external beam treatment for lung cancer. The goal of the study was to quantify intra- and interfraction variations in patient motion and dose as well as to assess the feasibility of daily use of the RADPOS system during patient treatments. Significant deviations in external surface motion were found throughout the course of treatment that agreed with similar studies done elsewhere and which emphasized a need for continued position monitoring. The average measured dose values were in agreement with treatment plan dose 
calculations for the majority of points. It should be noted that when the delivered dose is less than $50 \mathrm{cGy}$, the uncertainty on standard sensitivity MOSFET measurements increases. This amplified measurement uncertainty along with inaccuracies in treatment plan calculations near the air-surface interface could cause the measurements and dose calculations to be more vulnerable to slight inaccuracies in positioning, limitations in the treatment planning calculations, and machine output variations. This study also demonstrated that the RADPOS system can be used to monitor inter- and intra-fraction variations in breathing patterns and to measure surface dose without any disruption to the treatment schedule or discomfort to patients.

The final chapter of this thesis reviews a second clinical trial conducted using a modified RADPOS system. Measurements were made using a RADPOS detector with a MOSFET array that provided dose measurements at five different points, spaced $2 \mathrm{~cm}$ apart. The detector was positioned inside patients' urethra during seed implants for transperineal interstitial permanent prostate brachytherapy. Position and dose measurements were taken during the implantation procedure and for a period of time after all seeds were in place. Deviations from calculated treatment plan values were noted, leading to the suggestion that in vivo dosimetry should be used to verify actual delivered dose. Changes in position due to needle placement, swelling, or other internal motion as well as changes due to the TRUS probe were found to be significant in several cases and should therefore be quantified to evaluate influence on dose distributions. 


\title{
APPENDIX A
}

\author{
Pettent Luformution and Conent Form
}

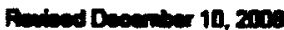

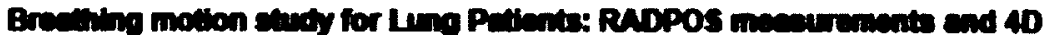

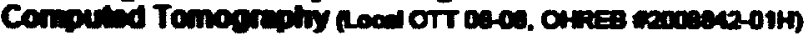

\section{mmosuction}

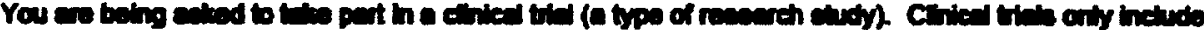

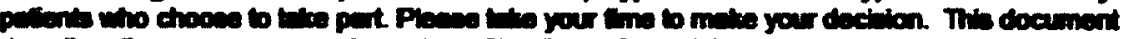

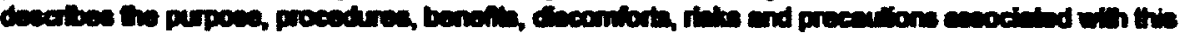

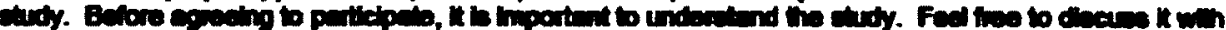

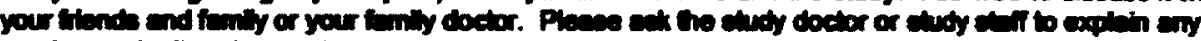

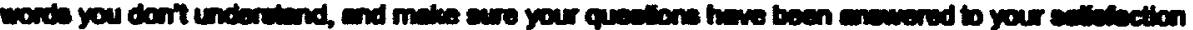

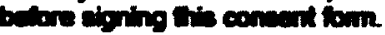

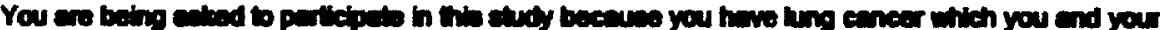

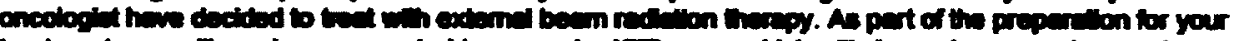

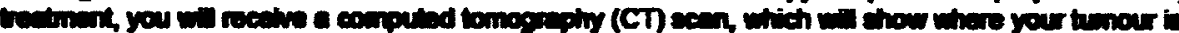

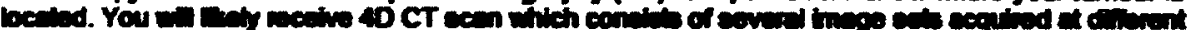

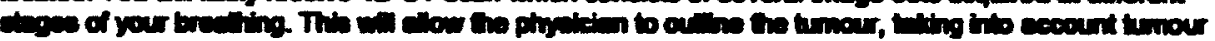

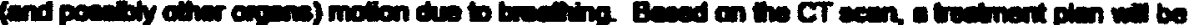

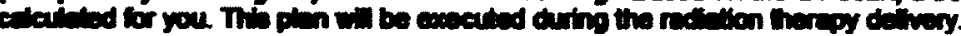

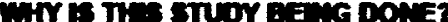

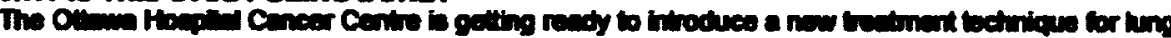

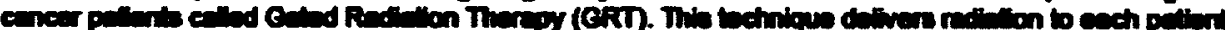

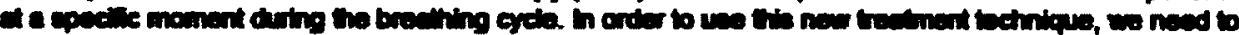

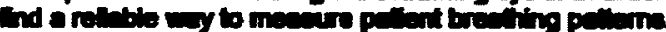

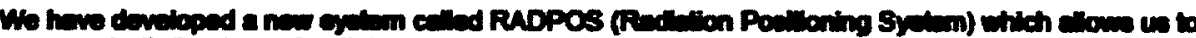

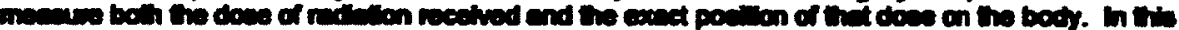

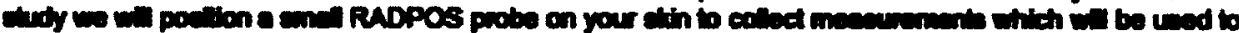

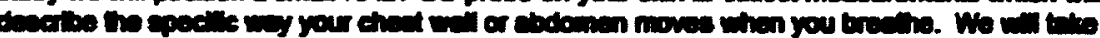

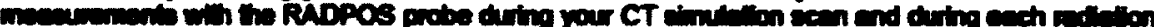

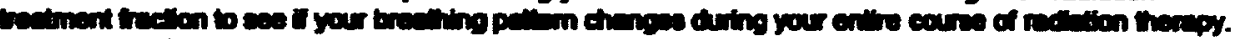

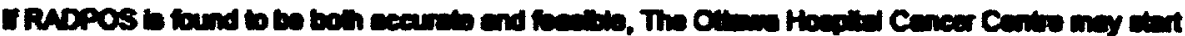

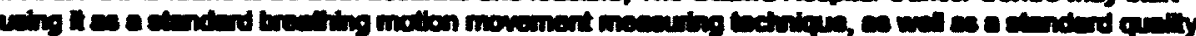

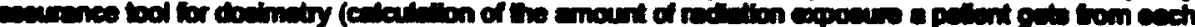
adothorgy tementh 


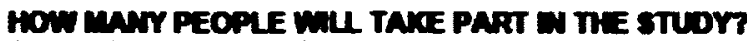

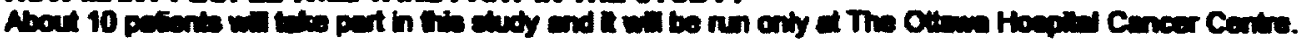

\section{DESTEN OF ThE STLOY}

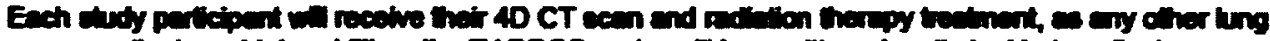

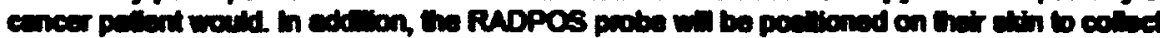

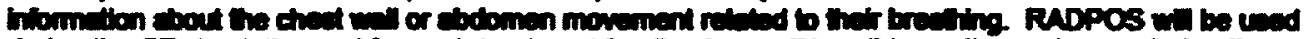

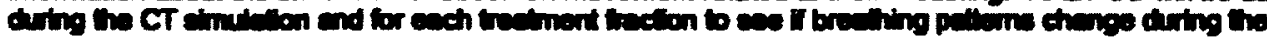

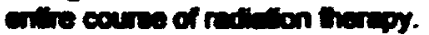

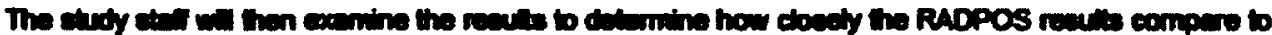

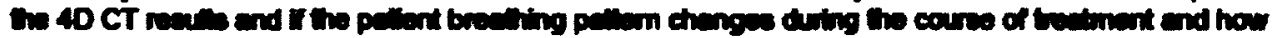
ingrimeant is the change.

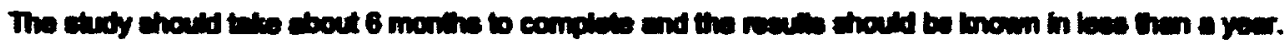

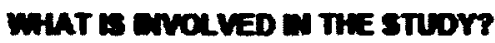

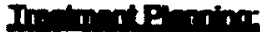

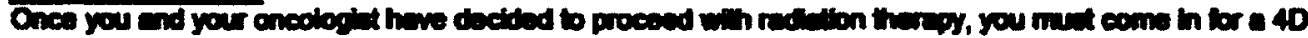

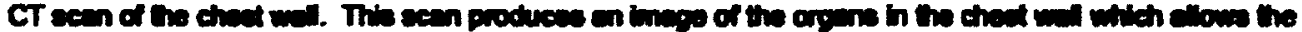

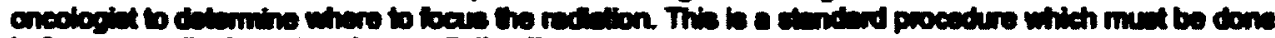

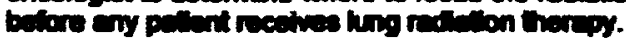

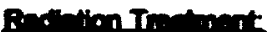

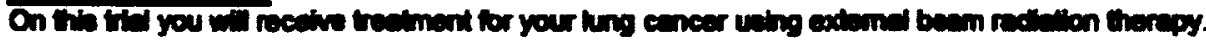

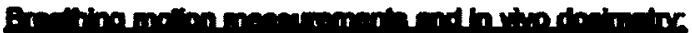

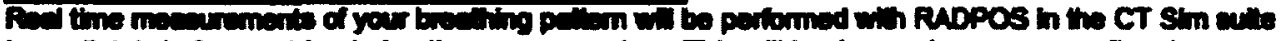

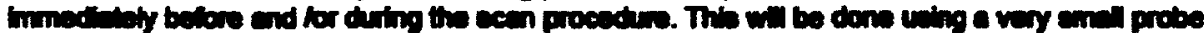

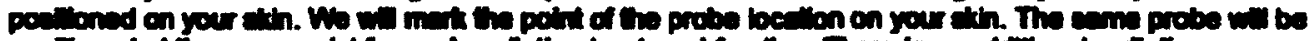

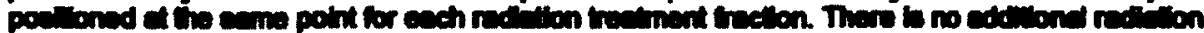

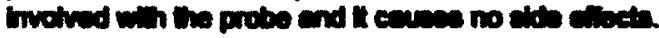

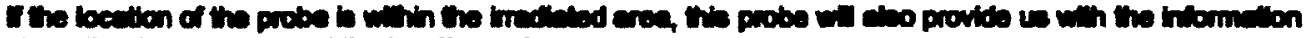
cbout the dave recelved et the locillon point.

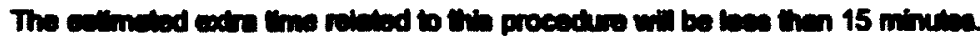

Ex.?.1\%:

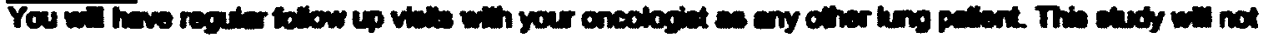

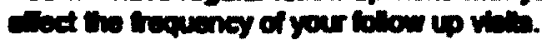

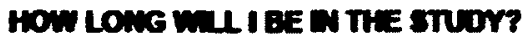

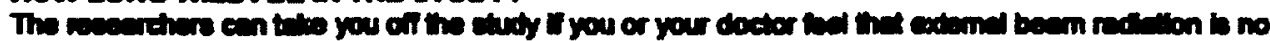
longor the bect trencinent for you.

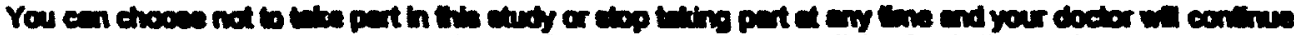

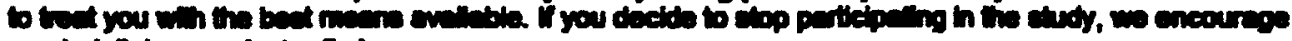
rou b the to your doctor bet 
WHAT ADE THE parks of THE STUOT?

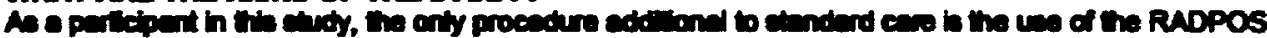

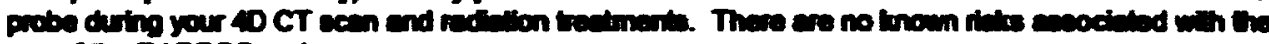
use of tha RADPOS pobe.

\section{ARE THERE EangTाS TO TAMUG PART W THE STUOV?}

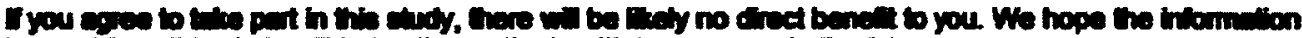

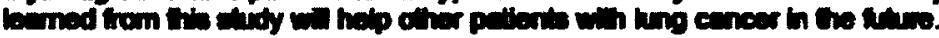

UHAT OTHER OPTOM⿻ ARE THERE?

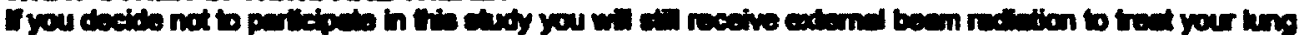
caner.

\section{MUAT ABOUT ConfinentuUTr?}

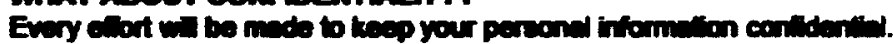

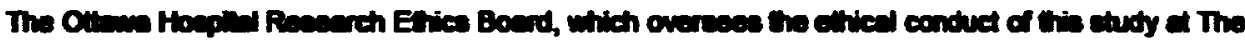

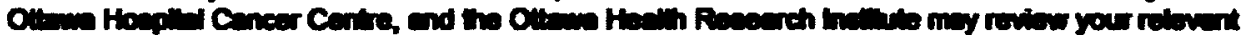

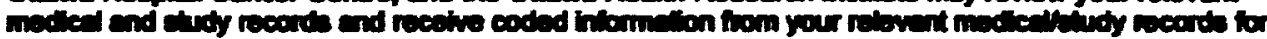

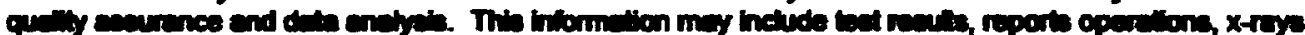

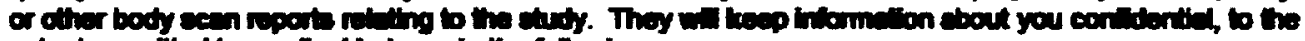

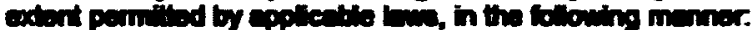

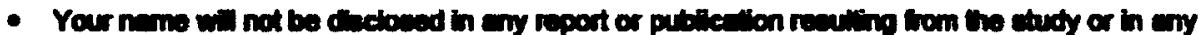

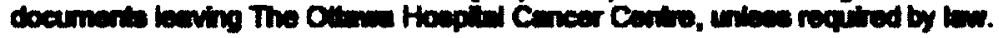

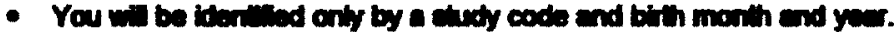

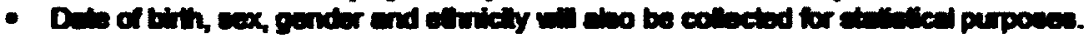

WHAT ADE THE COSTS?

You wil not be peid tor thing pent in the endy. There un be no cont to you for the use of the RADPOS

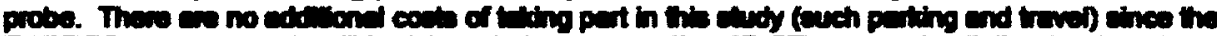

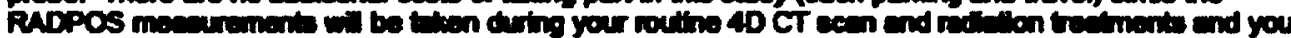

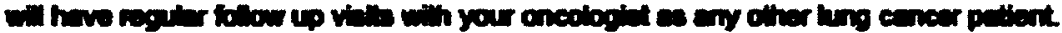

WHAT ABOUT Compenatrour?

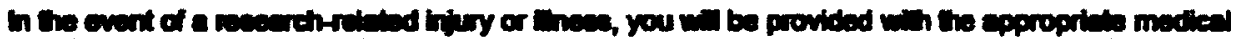

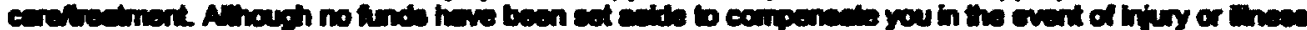

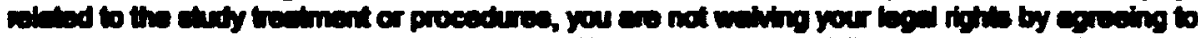

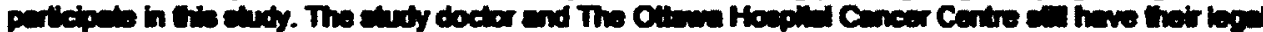

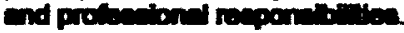

WHAT ARE UT RUETT AS A PARTCPANT?

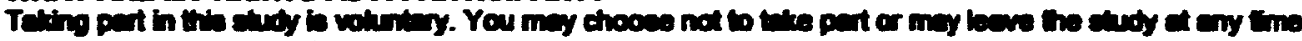

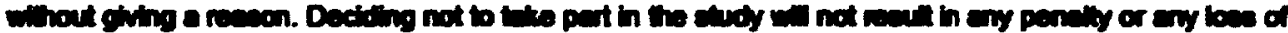

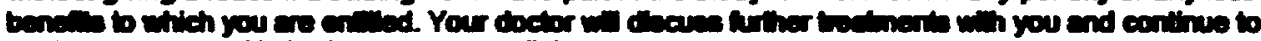

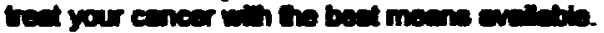

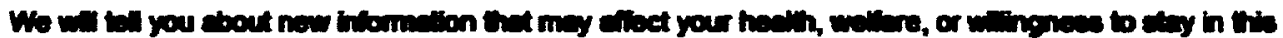
ardy. 
WHO DO I CALL IF I HAVE QUESTIONS OR PROBLEMS?

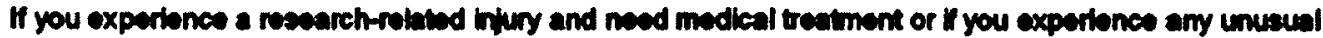
symploms or side cifects, you should inmediatbly contect your study doetor or the physictan reaponsible for this study, Dr. Gad Parry at 613-737-7700, Ext. 70215. If you noed to contact someone aner regular ctinic hours. ploase call 613-737-7700 and ask for the radiation oncologiat on call.

This protocol has been approwed by The Ottwwa Howpital Reaserch Ethics Board. This Board considers the ethical aspects of all hospilal research projects using human subiects. If you have any quections concerning this study or your rights as a research subject, you may contact eny of the following people:

- The Vice-President of The Otewa Hospital Cancer Centre, 613-737-7700. Ext. 70260.

- The Chairperson of The Oturwa Hospliti Research Ethics Board, 613-786-665, Ext. 14902.

\section{SIOMATURES}

My signature below means that I have read this 4 page pationt information and consent form, (or is has beon read to $\mathrm{mo}$ ). I have had the opportunity to roviow any quections with my study doctor. I agree to participats in this study and coneont to the sccess to my modical nconds as explained abows. $A$ copy of this pationt information and consent form will be given to me. Even though I have signed this consent form I can decide to withdraw from this study and withdraw permiscion for information about my progross to be collected. If I decide to do this, I will inform my study doctor, who will make a witien record of my deciaion.

Patient's Name (Pleace Print)

Patient's Signature

Name of ImvethatoriPerson Conducting the Informed Consent Discusalon (Pleases Primt)

Signature of ImvedigatoriPerson Conducting the intormed Consent Discusaion

\section{Dabe}

\section{Date}




\title{
APPENDIX B
}

\author{
Patient information and Consent Form
}

Roviand January 5. 2010

4D In Vwo Dosimetry Technique for Prostate Brachytherapy: RADpOS Dosinnetry and Competed Tomocraphy-Based Doshmetry (Local OTT-08-06, OHREB w200sen1-01H)

\section{MTRODUCTION}

You are being celbad to bake part in a dinical trial (o type of research study). Clinical triabs only include

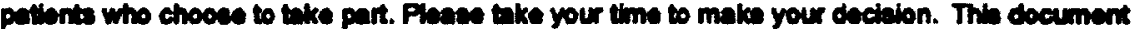

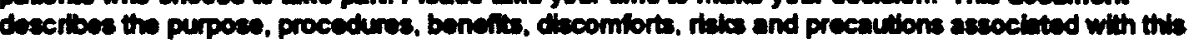

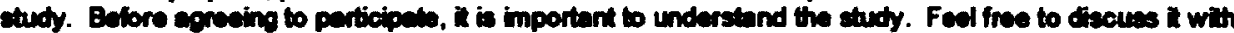
your friends and family or your faminy doctor. Plonse ask the study doctor or study stant to explein any words you dont understand. and make sure your questions have been answernd to your satialuction before sioning this consent form.

You are being asked to participeto in this study because you have low isk prostats cancer which you and your oncologint have decided to thent with brechythernpy. Brachytherapy is a radiation treatmont which involves pheing radlosetwo matertal directly in or near the produts. Uaully, abouk one month

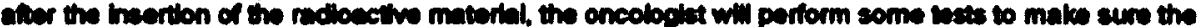
procedire was dons correctly. Ueunlly this involwes takting a compuled bomography (CT) sean which

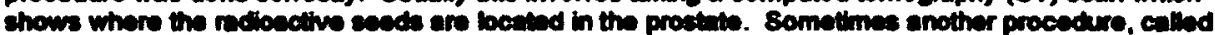
MOSFET (Metat-axida-semiconductor field-efect transiefor) is abo uaed. This procedure involves ineorting e catheser bito the urethre slong with a probe which measures redietion dose and position in the

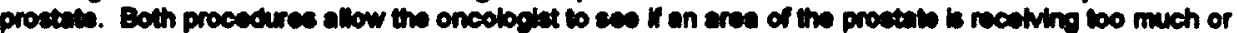
too livite rediation. This process of quality assurance is callod in vivo 'dosimetry'.

MHY is THAS STUOY BEWNO DONE?

AI The Oumwa Hosplual Cancor Cantre, the CT scan is our standard method of dosimetry. Dosimnetry is a

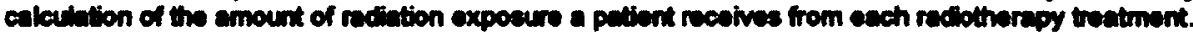
However, sometimes the MOSFET procedure is performed as well to comploment the reaclits. The problem with the CT procedture is that it is dons a month aller the brechythesapy. This meane thet if the

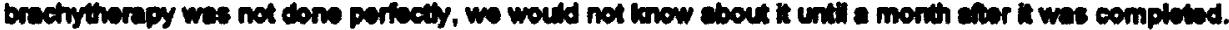

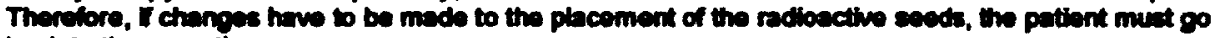
back to the operating room.

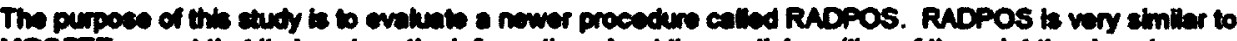

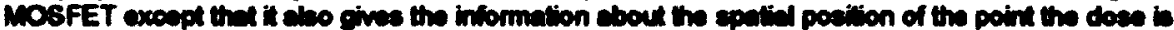
moseured. $K$ is dons at the seme time es the brechytherapy, whib the petiont is atil in the operniting room. During brecty themper. there is ahways a caltheler in the wrethre. The RADPOS detector is

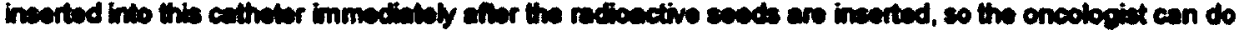

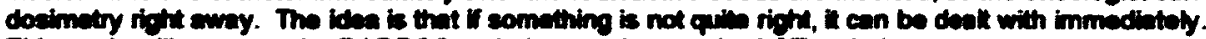
This study will compare the RADPOS technigue to the stendard CT Ecchriqu.

If RADPOS is found to be both scournte and baskble, 14 may become a standard dockmetry qually sesurence bechnique at The Ottowa Hosplital Cancer Centre. 
HOW MANY PEOPLE WUL TAKE PART W THE STUDY?

About 10 patients will take pert in this study and it will be run only at The Ottawa Hoapital Cencer Centre.

\section{DESIGN OF THE STUDY}

Each study participant will receive their brachytherepy treatment, as any other prodble cancer patient would. While the pentient is stit in the operating rocm for brechytherapy. RADPOS mesesurements will be performod.

Approximately 4 woeks after brachycherepy treatmont, study participents wil retum to the hoepial for dotimetry. 23 any other patient would. The standard CT dosimetry will be done, but RADPOS wil abo be done for a second time. The study steff will then examine each of the scans to determine how clasely the RADPOS results compare to the CT results.

The study should take about 1 year to complete and the reauts should be known in abouk 1 1/2 years.

WHAT IS INOC VED WN THE STUDY?

Tromententing:

Once you and your oncologias have decided to proceed with brectytherepy. you muat come in for treatument planning'. The procedure is callod TRUS (trenerected uliraconography), and iwolves incerting a probe into the rectum, which produces uttresound weves. This produces an imege of the organes in the pelvis which allows the oncologiat to determine where the radionctive meterial stould be placed to obtain the beot reouls. This is a standard procedure which muet be done before any petient receives brachytherapy.

Bractuthar oy Inetment:

On this trial you will receive treetment for your prostate cancer using prodtate bractytherapy. In proetate brachytherepy, rediation therepy is delivered by placing redioective "seeda" in and around your prostats. To treat the cancer, the radioective saeds emit a very low energy radiation, which is prinarity abeorbed in

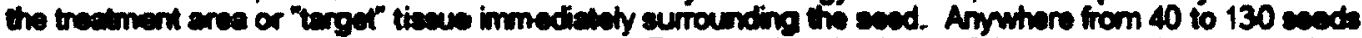
may be implented throughout the entire proetele. The celaulated volum of the proetate determines the

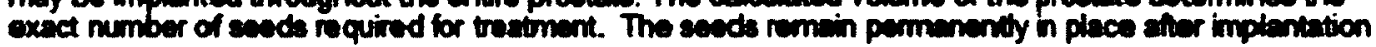
although they gredually lose their rediosctivity over time. Over $98 \%$ of the redionctivity is gone by the and of 12 monthe

Doninetre

RADPOS will be performed during this study. A will occur in the opereting room, immedielaly after brectyytherapy is completed. The RADPOS detector will be insented into the catheter which was alreecty put in place for the brachytherapy. Therefore, this RADPOS reacing does not involve ineerting an extra catherer.

About 4 wecks anter your brechyonerepy tredtment, you will come in for doaimaty, to determine the quality of the implants. You will receive the standerd CT-beesed docimetry, which involves a CT scan, a dreot $x$-ray. and a polvic $x$-ray. This procedure will take sprocimately 15-20 minutes, and is coutine for all patients receiving prostib bractyduerepy.

Foltowno:

You will have regular follow up vieits with your oncologiat for at least five years. These follow up viaits are routine for all brachytherapy petiento. 
HOW LONO WLL I EE IN THE STUDY?

The researchers can take you off the sucty for reasons such as:

- You or your doctor no longer feal that brechytherapy is the best treatment for you

- Your doctor does not feal that having a catheter ineertad is in your best intereas

You can choose not to talos part in this study or atop taking part at any time and your doctor will continue

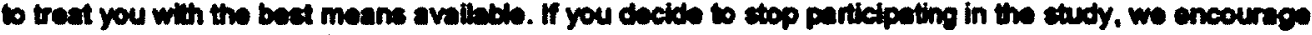
you to talk to your doctor fret.

You will have negular follow up with your radiation oncologiat for at least five years. If you stop baking part in the sudy, no further information will be collected as pert of this study alinough your doctor will atil be responalbis for your medionl care.

WHAT ARE THE RHKS OF THE STUDY?

As a participant in this study, you are required to undergo some teats and procedures which are additional to standard care. The riaks aceociatod with these addlional procedures are as follows:

RADPOS: no known riaks

Uninaryathetertation:

- Minor Alecomfort (60\%)

- Pain (requiring non-prescription analocicas) (<5\%)

- Urinary tract infection (\$S\%)

- Minor blesding (20\%)

ARE THERE BENEFITS TO TAKINO PART W THE STUOY?

If you chooes to particlpats in this etudy, thore will be likely no direct benefit to you. However chould the

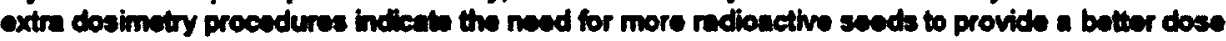
covernge, the phycicien may add extra secods.

In genoral, wo hope that the information barnod from this study will holp in post-imptant quality asturance for prostate cancer in the frture.

WHAT OTHER OPTONS ARE THERE?

If you decide not to participate in this study you will receive the standand CT-based dosimetry following your brectyytherapy treatunent and no immadiats or poet-implant dosinnetry with RADPOS.

You can recoive treatonont and follow-up assessment for your prostate cancer withouk being on this study. Piease talk to your doctor about these and other options.

WMT ABOUT CONFLENTALTY?

Every effort will be mads to keep your porsonal inflormation confidential.

The Ottom Hoepinal Recearch Ethics Board, which ovareess the ethical conduct of this study of The

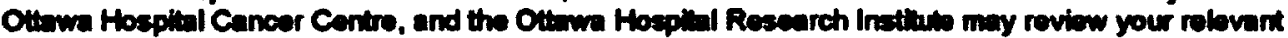
mectioal and atudy records and receive codad information from your rolovant medlealystudy records for quality escurance and date analycis. This information may inchude toet resuls, reports operations, $x$-rays or other body scan reports nelating to the suxdy. 
They will keep information about you confidential, to the extant permitied by applicable lans, in the following manner.

- Your name will not be cisclosed in eny report or publication reacling from the study or in any documents baving The Otume Hospitul Cancer Centre, unisess required by lew

- You will be identivied only by a study cods and bith month and year

- Date of birth, sex, sender and ethnicity will abo be collocted for efatietical purposes

WHAT ARE THE COSTS?

You will not be paid for taking part in this study. There will be no cost to you for the RADPOS detector. The additonal cots of taking part in this study (parking, trawl) are minimal since the RADPOS moasuremonts will be done on the same day ses your implam and egein on the sams day as the followup CT sean.

WHAT ABOUT COMPEMSATION?

In the event of a recearch-rebated iniury or ilinees, you will be provided wth the approprite modical treatment/care. Athough no funds have been sof aside to compensats you in the ovent of iniery or illness related to the study treatment or procedures, you are not waiving your bacal rights by apreaing to participate in this study. The study doctor and The Ottaws Hospital Canoer Centre sith have their logal and profecelonal reaponalbilitios.

WHAT ARE MY RUOHTS AS A PARTICIPANT?

Taking part in this study is voluntary. You may choose not to take part or may bave the study at amy time without giving a reason. Dociding not to bike part in the study will not result in any ponally or amy loes of benofits to which you are entited. Your doctor will discuse further treatments with you and continue bo treat your cencer with the best means avaitable.

We will tell you about new information that may affect your hoalth, wolfare, or willingness to stay in this study.

WHO DO I CALL IF I HAVE QUESTIONS OR PROELEMS?

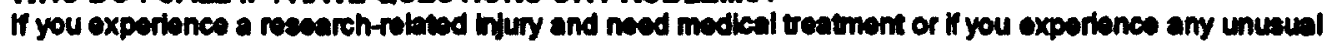
symploms or side effects, you should innmedintely contect your study doctor of the phricician responible for this study, Dr. Gad Parry at 613-737-7700. Ext. 70215. If you noed to contact comeone after recular clinic hours, please call 613-737-7700 and ask for the radiation oncologiat on call.

This protocol has been approved by The Oume Hospital Recearch Ethics Board. This Board considers the ethical aspects of all hoepilal research projects using human subjects. If you have any questions conceming this study or your fiobts as a ressarch subject, you may contact any of the following people:

- The Vice-President of The Ottrwa Hoepital Cancer Cantre, 613-737-7700, Ext. 70260

- The Chaipperson of The Otterwa Hospitul Research Ethics Board, 613-79e-6565, Ext. 14902 


\section{SHONATUPES}

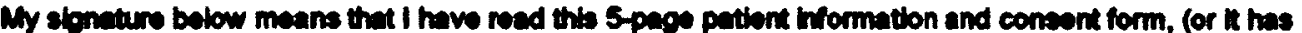

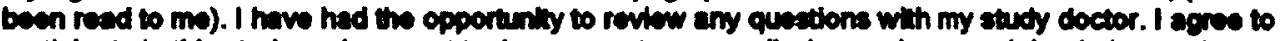

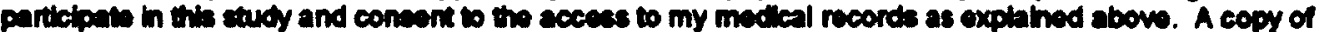
this petient information and consent form will be given to me. Even though I have sioned this convent form I can decide to withdraw from this study and withdraw permisaion for information about my progress to be colinctad. If I decide to do this. I will inform my study doctor, who will make a writion record of my decieion.

Pationt's Nams (Plases Print)

Pationt's Signature

Name of Inverientorf Person Conducting the

Informed Consent Diacusalon (Ploase Print)

Signature of Imvectiontor/Person Conducting the In ormed Consent Discussion
Dise

\section{Date}




\section{References}

1) Canadian Cancer Society's Steering Committee on Cancer Statistics, Canadian cancer statistics 2011, in: C.C. Society (Ed.), Toronto, ON, 2011.

2) B. Fraass, K. Doppke, M. Hunt, et al., American Association of Physicists in Medicine Radiation Therapy Committee Task Group 53: Quality assurance for clinical radiotherapy treatment planning, Med. Phys., 25 (1998) 1773-1829.

3) K.M. Langen, D.T.L. Jones, Organ motion and its management, Int. J. Radiat. Oncol. Biol. Phys., 50 (2001) 265-278.

4) D. Jones, ICRU report 50, prescribing, recording, and reporting photon beam therapy, in, Bethesda, Maryland, 1993.

5) S. Webb, Motion effects in (intensity modulated) radiation therapy: A review, Physics in Medicine and Biology, 51 (2006) R403-R425.

6) Y. Seppenwoolde, H. Shirato, K. Kitamura, et al., Precise and real-time measurement of $3 \mathrm{~d}$ tumor motion in lung due to breathing and heartbeat, measured during radiotherapy, Int. J. Radiat. Oncol. Biol. Phys., 53 (2002) 822-834.

7) M.G. Herman, R.A. Abrams, R.R. Mayer, Clinical use of online portal imaging for daily patient treatment verification, Int. J. Radiat. Oncol. Biol. Phys., 28 (1994) 10171023.

8) D.W. Litzenberg, J.M. Balter, S.W. Hadley, et al., Influence of intrafraction motion on margins for prostate radiotherapy, Int. J. Radiat. Oncol. Biol. Phys., 65 (2006) 548-553.

9) A.L. Wambersie, T., ICRU report 62, prescribing, recording, and reporting photon beam therapy (supplement to ICRU report 50), Bethesda, Maryland, 1999.

10) A. Chandra, L. Dong, E. Huang, et al., Experience of ultrasound-based daily prostate localization, Int. J. Radiat. Oncol. Biol. Phys., 56 (2003) 436-447.

11) T. Falco, G. Shenouda, C. Kaufmann, et al., Ultrasound imaging for external-beam prostate treatment setup and dosimetric verification, Medical dosimetry : official journal of the American Association of Medical Dosimetrists, 27 (2002) 271-273.

12) T.R. Willoughby, A.R. Forbes, D. Buchholz, et al., Evaluation of an infrared camera and $\mathrm{x}$-ray system using implanted fiducials in patients with lung tumors for gated radiation therapy, Int. J. Radiat. Oncol. Biol. Phys., 66 (2006) 568-575.

13) A. Ezz, P. Munro, A.T. Porter, et al., Daily monitoring and correction of radiationfield placement using a video-based portal imaging-system - a pilot-study, Int. J. Radiat. Oncol. Biol. Phys., 22 (1992) 159-165. 
14) D. Litzenberg, L.A. Dawson, H. Sandler, et al., Daily prostate targeting using implanted radiopaque markers, Int. J. Radiat. Oncol. Biol. Phys., 52 (2002) 699-703.

15) T.R. Mackie, J. Kapatoes, K. Ruchala, et al., Image guidance for precise conformal radiotherapy, Int. J. Radiat. Oncol. Biol. Phys., 56 (2003) 89-105.

16) L.A. Dawson, A.M. Balter, Interventions to reduce organ motion effects in radiation delivery, Semin. Radiat. Oncol., 14 (2004) 76-80.

17) P.J. Keall, G.S. Mageras, J.M. Balter, et al., The management of respiratory motion in radiation oncology: report of AAPM task group 76, Med. Phys., 33 (2006) 3874-3900.

18) J.Z. Wang, X.A. Li, W.D. D'Souza, R.D. Stewart, Impact of prolonged fraction delivery times on tumor control: A note of caution for intensity-modulated radiation therapy (imrt), Int. J. Radiat. Oncol. Biol. Phys., 57 (2003) 543-552.

19) T. Byrne, C. Ramsey, S. Mahan, D. Chase, The dosimetric impact of respiration motion on 3D, IMRT, and tomotherapy treatment delivery, Int. J. Radiat. Oncol. Biol. Phys., 63 (2005) S496-S496.

20) L.J. Forrest, T.R. Mackie, K. Ruchala, et al., The utility of megavoltage computed tomography images from a helical tomotherapy system for setup verification purposes, Int. J. Radiat. Oncol. Biol. Phys., 60 (2004) 1639-1644.

21) V.M. Remouchamps, D.P. Huyskens, I. Mertens, et al., The use of magnetic sensors to monitor moderate deep inspiration breath hold during breast irradiation with dynamic mlc compensators, Radiother. Oncol., 82 (2007) 341-348.

22) P.G. Seiler, H. Blattmann, S. Kirsch, R.K. Muench, C. Schilling, A novel tracking technique for the continuous precise measurement of tumour positions in conformal radiotherapy, Phys. Med. Biol., 45 (2000) N103-N110.

23) H.T. Chung, H.S. Jin, J.F. Dempsey, et al., Evaluation of surface and build-up region dose for intensity-modulated radiation therapy in head and neck cancer, Med. Phys., 32 (2005) 2682-2689.

24) E. Bloemen-van Gurp, W. du Bois, P. Visser, et al., Clinical dosimetry with mosfet dosimeters to determine the dose along the field junction in a split beam technique, Radiother. Oncol., 67 (2003) 351-357.

25) A. Saoudi, J.E. Cygler, R.W. Ashton, Dosimetry apparatus for e.G. Prostate cancer treatment has a computer that processes both radiation level and position signals via electronic interface and provides indication of radiation dose that is applied to treated tissue, Best Medical Canada Ltd (Best-Non-standard), pp. 13. 
26) M. Soubra, J. Cygler, G. Mackay, Evaluation of a dual bias dual metal-oxide-silicon semiconductor field-effect transistor detector as radiation dosimeter, Med. Phys., 21 (1994) 567-572.

27) J.B. Hummel, M.R. Bax, M.L. Figl, et al., Design and application of an assessment protocol for electromagnetic tracking systems, Med. Phys., 32 (2005) 2371-2379.

28) A. Viamonte, L.A.R. da Rosa, L.A. Buckley, A. Cherpak, J.E. Cygler, Radiotherapy dosimetry using a commercial osl system, Med. Phys., 35 (2008) 1261-1266.

29) M. Essers, B.J. Mijnheer, In vivo dosimetry during external photon beam radiotherapy, Int. J. Radiat. Oncol. Biol. Phys., 43 (1999) 245-259.

30) D.J. Gladstone, X.Q. Lu, J.L. Humm, H.F. Bowman, L.M. Chi, A miniature mosfet radiation dosimeter probe, Med. Phys., 21 (1994) 1721-1728.

31) P. Scalchi, P. Francescon, P. Rajaguru, Characterization of a new MOSFET detector configuration for in vivo skin dosimetry, Med. Phys., 32 (2005) 1571-1578.

32) R. Ramaseshan, K.S. Kohli, T.J. Zhang, et al., Performance characteristics of a micromosfet as an in vivo dosimeter in radiation therapy, Phys. Med. Biol., 49 (2004) 4031-4048.

33) M.J. Butson, A. Rozenfeld, J.N. Mathur, et al., A new radiotherapy surface dose detector: The MOSFET, Med. Phys., 23 (1996) 655-658.

34) P. Scalchi, P. Francescon, Calibration of a MOSFET detection system for 6-MV in vivo dosimetry, Int. J. Radiat. Oncol. Biol. Phys., 40 (1998) 987-993.

35) S. Marcie, E. Charpiot, R.J. Bensadoun, et al., In vivo measurements with MOSFET detectors in oropharynx and nasopharynx intensity-modulated radiation therapy, Int. $J$. Radiat. Oncol. Biol. Phys., 61 (2005) 1603-1606.

36) C.F. Chuang, L.J. Verhey, P. Xia, Investigation of the use of MOSFET for clinical IMRT dosimetric verification, Med. Phys., 29 (2002) 1109-1115.

37) A. Cherpak, R.C.N. Studinski, J.E. Cygler, MOSFET detectors in quality assurance of tomotherapy treatments, Radiother. Oncol., 86 (2008) 242-250.

38) R. Ramani, S. Russell, P. Obrien, Clinical dosimetry using MOSFET, Int. J. Radiat. Oncol. Biol. Phys., 37 (1997) 959-964.

39) N. Jornet, P. Carrasco, D. Jurado, et al., Comparison study of MOSFET detectors and diodes for entrance in vivo dosimetry in $18 \mathrm{MV}$ x-ray beams, Med. Phys., 31 (2004) 2534-2542. 
40) A.D. Milne, D.G. Chess, J.A. Johnson, G.J.W. King, Accuracy of an electromagnetic tracking device: A study of the optimal operating range and metal interference, J. Biomech., 29 (1996) 791-793.

41) J.M. Balter, J.N. Wright, L.J. Newell, et al., Accuracy of a wireless localization system for radiotherapy, Int. J. Radiat. Oncol. Biol. Phys., 61 (2005) 933-937.

42) J. Hummel, M. Figl, C. Kollmann, H. Bergmann, W. Birkfellner, Evaluation of a miniature electromagnetic position tracker, Med. Phys., 29 (2002) 2205-2212.

43) P.R. Almond, P.J. Biggs, B.M. Coursey, et al., AAPM's TG-51 protocol for clinical reference dosimetry of high-energy photon and electron beams, Med. Phys., 26 (1999) 1847-1870.

44) Z.H. Yin, R.P.; and Beddoe, A.H., Response of silicon diode dosemeters to scattered radiation from megavoltage photon beams, Radiat. Prot. Dosim., 101 (2002) 414-418.

45) G. Rikner, Characteristics of a selectively shielded p-si detector in Co-60 and 8 and 16 MV roentgen radiation, Acta Radiol. Oncol., 24 (1985) 205-208.

46) J.E. and .S.P. Cygler, Mosfet dosimetry in radiotherapy, in: D.W.O. Rogers and J.E. Cygler (Ed.) Clinical dosimetry measurements in radiotherapy. Medical Physics Publishing, Madison, Wisconsin, 2010, pp. 941-977.

47) A. Cherpak, W. Ding, A. Hallil, J.E. Cygler, Evaluation of a novel 4D in vivo dosimetry system, Med. Phys., 36 (2009) 1672-1679.

48) M.K. Martel, R.K. Ten Haken, M.B. Hazuka, et al., Estimation of tumor control probability model parameters from 3-D dose distributions of non-small cell lung cancer patients, Lung Cancer, 24 (1999) 31-37.

49) M. Machtay, C. Hsu, R. Komaki, et al., Effect of overall treatment time on outcomes after concurrent chemoradiation for locally advanced non-small-cell lung carcinoma: Analysis of the radiation therapy oncology group (rtog) experience, Int. J. Radiat. Oncol. Biol. Phys., 63 (2005) 667-671.

50) S.L.S. Kwa, J.V. Lebesque, J.C.M. Theuws, et al., Radiation pneumonitis as a function of mean lung dose: An analysis of pooled data of 540 patients, Int. J. Radiat. Oncol. Biol. Phys., 42 (1998) 1-9.

51) M.V. Graham, J.A. Purdy, B. Emami, et al., Clinical dose-volume histogram analysis for pneumonitis after 3D treatment for non-small cell lung cancer (NSCLC), Int. J. Radiat. Oncol. Biol. Phys., 45 (1999) 323-329. 
52) M.L. Hernando, L.B. Marks, G.C. Bentel, et al., Radiation-induced pulmonary toxicity: A dose-volume histogram analysis in 201 patients with lung cancer, Int. J. Radiat. Oncol. Biol. Phys., 51 (2001) 650-659.

53) D. Oetzel, P. Schraube, F. Hensley, et al., Estimation of pneumonitis risk in 3dimensional treatment planning using dose-volume histogram analysis, Int. J. Radiat. Oncol. Biol. Phys., 33 (1995) 455-460.

54) Y. Seppenwoolde, J.V. Lebesque, K. de Jaeger, et al., Comparing different NTCP models that predict the incidence of radiation pneumonitis, Int. J. Radiat. Oncol. Biol. Phys., 55 (2003) 724-735.

55) E.D. Yorke, A. Jackson, K.E. Rosenzweig, et al., Dose-volume factors contributing to the incidence of radiation pneumonitis in non-small-cell lung cancer patients treated with three-dimensional conformal radiation therapy, Int. J. Radiat. Oncol. Biol. Phys., 54 (2002) 329-339.

56) S.S. Vedam, P.J. Keall, V.R. Kini, et al., Acquiring a four-dimensional computed tomography dataset using an external respiratory signal, Phys. Med. Biol., 48 (2003) 4562.

57) T. Neicu, R. Berbeco, J. Wolfgang, S.B. Jiang, Synchronized moving aperture radiation therapy (SMART): Improvement of breathing pattern reproducibility using respiratory coaching, Phys. Med. Biol., 51 (2006) 617-636.

58) R. George, P.J. Keall, V.R. Kini, et al., Is the diaphragm motion probability density function normally distributed?, Med. Phys., 32 (2005) 396-404.

59) S.S. Vedam, P.J. Keall, A. Docef, et al., Predicting respiratory motion for fourdimensional radiotherapy, Med. Phys., 31 (2004) 2274-2283.

60) P. Keall, 4-dimensional computed tomography imaging and treatment planning, Semin. Radiat. Oncol., 14 (2004) $81-90$.

61) W.D. D'Souza, Y. Kwok, C. Deyoung, et al., Gated CT imaging using a freebreathing respiration signal from flow-volume spirometry, Med. Phys., 32 (2005) 3641 3649.

62) W.G. Lu, M.L. Chen, G.H. Olivera, K.J. Ruchala, T.R. Mackie, Fast free-form deformable registration via calculus of variations, Phys. Med. Biol., 49 (2004) 30673087.

63) E.C. Ford, G.S. Mageras, E. Yorke, C.C. Ling, Respiration-correlated spiral CT: A method of measuring respiratory-induced anatomic motion for radiation treatment planning, Med. Phys., 30 (2003) 88-97. 
64) E. Rietzel, T.S. Pan, G.T.Y. Chen, Four-dimensional computed tomography: Image formation and clinical protocol, Med. Phys., 32 (2005) 874-889.

65) H. Alasti, Y.B. Cho, A.D. Vandermeer, et al., A novel four-dimensional radiotherapy method for lung cancer: Imaging, treatment planning and delivery, Phys. Med. Biol., 51 (2006) 3251-3267.

66) D.S. Followill, D.R. Evans, C. Cherry, et al., Design, development, and implementation of the radiological physics center's pelvis and thorax anthropomorphic quality assurance phantoms, Med. Phys., 34 (2007) 2070-2076.

67) P.J. Keall, V.R. Kini, S.S. Vedam, R. Mohan, Motion adaptive x-ray therapy: A feasibility study, Phys. Med. Biol., 46 (2001) 1-10.

68) T. Li, E. Schreibmann, B. Thorndyke, et al., Radiation dose reduction in fourdimensional computed tomography, Med. Phys., 32 (2005) 3650-3660.

69) E. Nioutsikou, J.R.N. Symonds-Tayler, J.L. Bedford, S. Webb, Quantifying the effect of respiratory motion on lung tumour dosimetry with the aid of a breathing phantom with deforming lungs, Phys. Med. Biol., 51 (2006) 3359-3374.

70) Y.Y. Vinogradskiy, P. Balter, D.S. Followill, et al., Comparing the accuracy of fourdimensional photon dose calculations with three-dimensional calculations using moving and deforming phantoms, Med. Phys., 36 (2009) 5000-5006.

71) Y.Y. Vinogradskiy, P. Balter, D.S. Followill, et al., Verification of four-dimensional photon dose calculations, Med. Phys., 36 (2009) 3438-3447.

72) R. Kashani, M. Hub, M.L. Kessler, J.M. Balter, Technical note: A physical phantom for assessment of accuracy of deformable alignment algorithms, Med. Phys., 34 (2007) 2785-2788.

73) R. Kashani, K. Lam, D. Litzenberg, J. Balter, Technical note: A deformable phantom for dynamic modeling in radiation therapy, Med. Phys., 34 (2007) 199-201.

74) S.B. Jiang, C. Pope, K.M. Al Jarrah, et al., An experimental investigation on intrafractional organ motion effects in lung imrt treatments, Phys. Med. Biol., 48 (2003) 17731784.

75) M. Serban, E. Heath, G. Stroian, D.L. Collins, J. Seuntjens, A deformable phantom for $4 \mathrm{D}$ radiotherapy verification: Design and image registration evaluation, Med. Phys., 35 (2008) 1094-1102.

76) C.J. Haasbeek, B.J. Slotman, S. Senan, Radiotherapy for lung cancer: Clinical impact of recent technical advances, Lung Cancer, 64 (2009) 1-8. 
77) J.D. Hoisak, K.E. Sixel, R. Tirona, P.C. Cheung, J.P. Pignol, Correlation of lung tumor motion with external surrogate indicators of respiration, Int. J. Radiat. Oncol. Biol. Phys., 60 (2004) 1298-1306.

78) Q.S. Chen, M.S. Weinhous, F.C. Deibel, J.P. Ciezki, R.M. Macklis, Fluoroscopic study of tumor motion due to breathing: Facilitating precise radiation therapy for lung cancer patients, Med. Phys., 28 (2001) 1850-1856.

79) G. Hugo, C. Vargas, J. Liang, et al., Changes in the respiratory pattern during radiotherapy for cancer in the lung, Radiother. Oncol., 78 (2006) 326-331.

80) L. Ekberg, O. Holmberg, L. Wittgren, G. Bjelkengren, T. Landberg, What margins should be added to the clinical target volume in radiotherapy treatment planning for lung cancer?, Radiother. Oncol., 48 (1998) 71-77.

81) J. Mechalakos, E. Yorke, G.S. Mageras, et al., Dosimetric effect of respiratory motion in external beam radiotherapy of the lung, Radiother. Oncol., 71 (2004) 191-200.

82) M. Engelsmann, E.M.F. Damen, K. De Jaeger, K.M. van Ingen, B.J. Mijnheer, The effect of breathing and set-up errors on the cumulative dose to a lung tumor, Radiother. Oncol., 60 (2001) 95-105.

83) N.C. van der Voort van Zyp, J.-B. Prevost, M.S. Hoogeman, et al., Stereotactic radiotherapy with real-time tumor tracking for non-small cell lung cancer: Clinical outcome, Radiother. Oncol., 91 (2009) 296-300.

84) S. Yousefi, B.I. Collins, C.A. Reichner, et al., Complications of thoracic computed tomography-guided fiducial placement for the purpose of stereotactic body radiation therapy, Clin. Lung Cancer, 8 (2007) 252-256.

85) T.Z. Zhang, H. Keller, M.J. O'Brien, T.R. Mackie, B. Paliwal, Application of the spirometer in respiratory gated radiotherapy, Med. Phys., 30 (2003) 3165-3171 .

86) V.R.V. Kini, S.S. Keall, P.J.; and Mohan, A.R., A dynamic non-invasive technique for predicting organ motion in respiratory-gated radiotherapy of the chest., Int. J. Radiat. Oncol. Biol. Phys., 51 (2001) 25-26.

87) S.S. Vedam, P.J. Keall, V.R. Kini, R. Mohan, Determining parameters for respiration-gated radiotherapy, Med. Phys., 28 (2001) 2139-2146.

88) D.P. Gierga, J. Brewer, G.C. Sharp, et al., The correlation between internal and external markers for abdominal tumors: Implications for respiratory gating, Int. J. Radiat. Oncol. Biol. Phys., 61 (2005) 1551-1558. 
89) T. Juhler Nottrup, S.S. Korreman, A.N. Pedersen, et al., Intra- and interfraction breathing variations during curative radiotherapy for lung cancer, Radiother. Oncol., 84 (2007) 40-48.

90) R.I. Berbeco, S. Nishioka, H. Shirato, G.T. Chen, S.B. Jiang, Residual motion of lung tumours in gated radiotherapy with external respiratory surrogates, Phys Med Biol, 50 (2005) 3655-3667.

91) Y. Tsunashima, T. Sakae, Y. Shioyama, et al., Correlation between the respiratory waveform measured using a respiratory sensor and 3D tumor motion in gated radiotherapy, Radiother. Oncol., 60 (2004) 951-958.

92) J.D. Hoisak, K.E. Sixel, R. Tirona, P.C. Cheung, J.P. Pignol, Prediction of lung tumour position based on spirometry and on abdominal displacement: Accuracy and reproducibility, Radiother. Oncol., 78 (2006) 339-346.

93) S. Korreman, H. Mostafavi, A. Grow, A. Boyer, Q. Le, Fluoroscopic characterization of lung tumor breathing motion during respiratory gating, Int. J. Radiat. Oncol. Biol. Phys., 60 (2004) S287-S288.

94) F.O. Spoelstra, J.R. van Sornsen de Koste, J.P. Cuijpers, et al., Analysis of reproducibility of respiration-triggered gated radiotherapy for lung tumors, Radiother. Oncol., 87 (2008) 59-64.

95) A. Cherpak, M. Serban, J. Seuntjens, J.E. Cygler, 4d dose-position verification in radiation therapy using the RADPOS system in a deformable lung phantom, Med. Phys., 38 (2011) 179-187.

96) P. Keall, S. Vedam, R. George, et al., The clinical implementation of respiratorygated intensity-modulated radiotherapy, Med. Dosim., 31 (2006) 152-162.

97) J. Kindblom, A.-M. Ekelund-Olvenmark, H. Syren, et al., High precision transponder localization using a novel electromagnetic positioning system in patients with localized prostate cancer, Radiother. Oncol., 90 (2009) 307-311.

98) K.M. Forster, C.W. Stevens, K. Kitamura, et al., Changes of tumor motion patterns during a course of radiation therapy for lung cancer, Int. J. Radiat. Oncol. Biol., 57 (2003) S234.

99) G. Bosmans, A. van Baardwijk, A. Dekker, et al., Intra-patient variability of tumor volume and tumor motion during conventionally fractionated radiotherapy for locally advanced non-small-cell lung cancer: A prospective clinical study, Int. J. Radiat. Oncol. Biol., 66 (2006) 748-753.

100) K.J. Redmond, D.Y. Song, J.L. Fox, et al., Respiratory motion changes of lung tumors over the course of radiation therapy based on respiration-correlated four- 
dimensional computed tomography scans, Int. J. Radiat. Oncol. Biol., 75 (2009) 16051612.

101) J.H. Killoran, A.M. Allen, B.H. Kann, Y. Lyatskaya, Inter fractional variability of breathing phase definition as determined by fiducial location, Med. Phys., 35 (2008) 753763.

102) T. Neicu, et al., Synchronized moving aperture radiation therapy (smart): Average tumour trajectory for lung patients, Phys. Med. Biol., 48 (2003) 587.

103) A. Schweikard, G. Glosser, M. Bodduluri, M.J. Murphy, J.R. Adler, Robotic motion compensation for respiratory movement during radiosurgery, Comp. Surg., 5 (2000) 263277.

104) N. Koch, H.H. Liu, G. Starkschall, et al., Evaluation of internal lung motion for respiratory-gated radiotherapy using mri: Part i--correlating internal lung motion with skin fiducial motion, Int. J. Radiat. Oncol. Biol., 60 (2004) 1459-1472.

105) R.I. Berbeco, et al., Residual motion of lung tumours in gated radiotherapy with external respiratory surrogates, Phys. Med. Biol., 50 (2005) 3655.

106) W. Hamilton, D. Sharp, Symptomatic diagnosis of prostate cancer in primary care: A structured review, Br. J. Gen. Pract., 54 (2004) 617-621.

107) O. Bratt, A. Berglund, J. Adolfsson, et al., Prostate cancer diagnosed after prostatespecific antigen testing of men without clinical signs of the disease: A population-based study from the national prostate cancer register of sweden, Scand. J. Urol. Nephrol., 44 (2010) 384-390.

108) W.J. Catalona, D.S. Smith, T.L. Ratliff, et al., Measurement of prostate-specific antigen in serum as a screening-test for prostate-cancer, N. Engl. J. Med., 324 (1991) 1156-1161.

109) Y. Yu, L.L. Anderson, Z.F. Li, et al., Permanent prostate seed implant brachytherapy: Report of the American Association of Physicists in Medicine Task Group No. 64, Med. Phys., 26 (1999) 2054-2076.

110) P.A. Abrahamsson, Prostate cancer: To treat or not to treat?, Eur. Urol. Suppl., 8 (2009) 418-423.

111) A. Sahgal, M. Roach, Permanent prostate seed brachytherapy: A current perspective on the evolution of the technique and its application, Nat. Clin. Pract. Urol., 4 (2007) 658-670.

112) H.H. Holm, The history of interstitial brachytherapy of prostatic cancer, Sem. Surg. Oncol., 13 (1997) 431-437. 
113) T.P. Mate, J.E. Gottesman, J. Hatton, M. Gribble, L. Van Hollebeke, High dose-rate afterloading (192)iridium prostate brachytherapy: Feasibility report, Int. J. Radiat. Oncol. Biol. Phys., 41 (1998) 525-533.

114) M.J. Ghilezan, D.A. Jaffray, J.H. Siewerdsen, et al., Prostate gland motion assessed with cine-magnetic resonance imaging (cine-mri), Int. J. Radiat. Oncol. Biol. Phys., 62 (2005) 406-417.

115) B. Liu, F.A. Lerma, S. Patel, et al., Dosimetric effects of the prone and supine positions on image guided localized prostate cancer radiotherapy, Radiother. Oncol., 88 (2008) 67-76.

116) D. Mah, G. Freedman, B. Milestone, et al., Measurement of intrafractional prostate motion using magnetic resonance imaging, Int. J. Radiat. Oncol. Biol. Phys., 54 (2002) 568-575.

117) J.M. Balter, H.M. Sandler, K. Lam, et al., Measurement of prostate movement over the course of routine radiotherapy using implanted markers, Int. J. Radiat. Oncol. Biol. Phys., 31 (1995) 113-118.

118) K.M. Langen, Y.S. Zhang, R.D. Andrews, et al., Initial experience with megavoltage (MV) CT guidance for daily prostate alignments, Int. J. Radiat. Oncol. Biol. Phys., 62 (2005) 1517-1524.

119) D.J. Moseley, E.A. White, K.L. Wiltshire, et al., Comparison of localization performance with implanted fiducial markers and cone-beam computed tomography for on-line image-guided radiotherapy of the prostate, Int. J. Radiat. Oncol. Biol. Phys., 67 (2007) 942-953.

120) E. Vigneault, J. Pouliot, J. Laverdiere, J. Roy, M. Dorion, Electronic portal imaging device detection of radioopaque markers for the evaluation of prostate position during megavoltage irradiation: A clinical study, Int. J. Radiat. Oncol. Biol. Phys., 37 (1997) 205-212.

121) J. Lattanzi, S. McNeeley, W. Pinover, et al., A comparison of daily CT localization to a daily ultrasound-based system in prostate cancer, Int. J. Radiat. Oncol. Biol. Phys., 43 (1999) 719-725.

122) J. Booth, Modelling the impact of treatment uncertainties in radiotherapy, University of Adelaide, Australia, 2002.

123) R.S. Sloboda, N. Usmani, J. Pedersen, et al., Time course of prostate edema post permanent seed implant determined by magnetic resonance imaging, Brachy., 9 (2010) 354-361. 
124) N.N. Stone, J. Roy, S. Hong, Y.-C. Lo, R.G. Stock, Prostate gland motion and deformation caused by needle placement during brachytherapy, Brachy., 1 (2002) 154160.

125) G.S. Merrick, W.M. Butler, J.H. Lief, A.T. Dorsey, Temporal resolution of urinary morbidity following prostate brachytherapy, Int. J. Radiat. Oncol. Biol. Phys., 47 (2000) 121-128.

126) M. Pinkawa, B. Gagel, B. Asadpour, et al., Seed displacements after permanent brachytherapy for prostate cancer in dependence on the prostate level, Strahlentherapie und Onkologie, 184 (2008) 520-525.

127) K.M. Langen, T.R. Willoughby, S.L. Meeks, et al., Observations on real-time prostate gland motion using electromagnetic tracking, Int. J. Radiat. Oncol. Biol. Phys., 71 (2008) 1084-1090.

128) R.P. Smith, S. Beriwall, K. Komanduri, E. Gibbons, R. Benoit, Planning based on postneedle volume with early dosimetric assessment is beneficial for cesium-131 permanent prostate seed implantation, Brachy., 7 (2008) 237-241.

129) G. Leclerc, M.C. Lavallee, R. Roy, E. Vigneault, L. Beaulieu, Prostatic edema in Ii125 permanent prostate implants: Dynamical dosimetry taking volume changes into account, Med. Phys., 33 (2006) 574-583.

130) T. Ohashi, A. Yorozu, K. Toya, et al., Comparison of intraoperative ultrasound with postimplant computed tomography-dosimetric values at day 1 and day 30 after prostate brachytherapy, Brachy., 6 (2007) 246-253.

131) Y. Yamada, L. Potters, M. Zaider, et al., Impact of intraoperative edema during transperineal permanent prostate brachytherapy on computer-optimized and preimplant planning techniques, Am. J. Clin. Oncol., 26 (2003) e130-e135.

132) V. Lagerburg, M.A. Moerland, J.J.W. Lagendijk, J.J. Battermann, Measurement of prostate rotation during insertion of needles for brachytherapy, Radiother. Oncol., 77 (2005) 318-323.

133) J.E. Cygler, A. Saoudi, G. Perry, C. Morash, C. E, Feasibility study of using mosfet detectors for in vivo dosimetry during permanent low-dose-rate prostate implants, Radiother. Oncol., 80 (2006) 296-301.

134) E.J. Bloemen-van Gurp, B.K.C. Haanstra, L.H.P. Murrer, et al., In vivo dosimetry with a linear MOSFET array to evaluate the urethra dose during permanent implant brachytherapy using iodine-125, Int. J. Radiat. Oncol. Biol. Phys., 75 (2009) 1266-1272.

135) I. Jurkovic, A. Sadeghi, B. Prestidge, et al., In vivo MOSFET detector used in the post-operative dose assessment of $\mathrm{i}-125$ prostate implants, Brachy., 5 (2006) 97. 
136) E.J. Bloemen-van Gurp, L.H.P. Murrer, B.K.C. Haanstra, et al., In vivo dosimetry using a linear MOSFET-array dosimeter to determine the urethra dose in (125)I permanent prostate implants, Int. J. Radiat. Oncol. Biol. Phys., 73 (2009) 314-321. 